\title{
Asymmetric 1,4-Michael Addition In Aqueous Medium Using Hydrophobic Chiral Organocatalysts
}

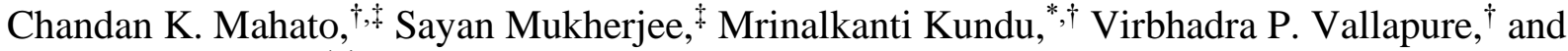
Animesh Pramanik ${ }^{*}$,

${ }^{\dagger}$ TCG Lifesciences Pvt. Ltd., BN-7, Sector V, Salt Lake City, Kolkata-700091, India.

Fepartment of Chemistry, University of Calcutta, 92, A. P. C. Road, Kolkata 700009, India.

E-mail: mrinal.kundu@tcgls.com,pramanikanimesh61@gmail.com

\section{Supporting Information}

\section{Table of Contents:}

NMR spectra of catalysts.

NMR spectra of Michael products.

NMR spectra of (3R,3aS,7aR)-3-(naphthalen-2-yl)octahydro-1H-indole (34a)

and (3R,3aS,7aS)-3-(naphthalen-2-yl)octahydro-1H-indole (34b)

Chiral HPLC chromatogram of Michael Products

S74-S90

Images of various stages of the reaction.

S91-S91 


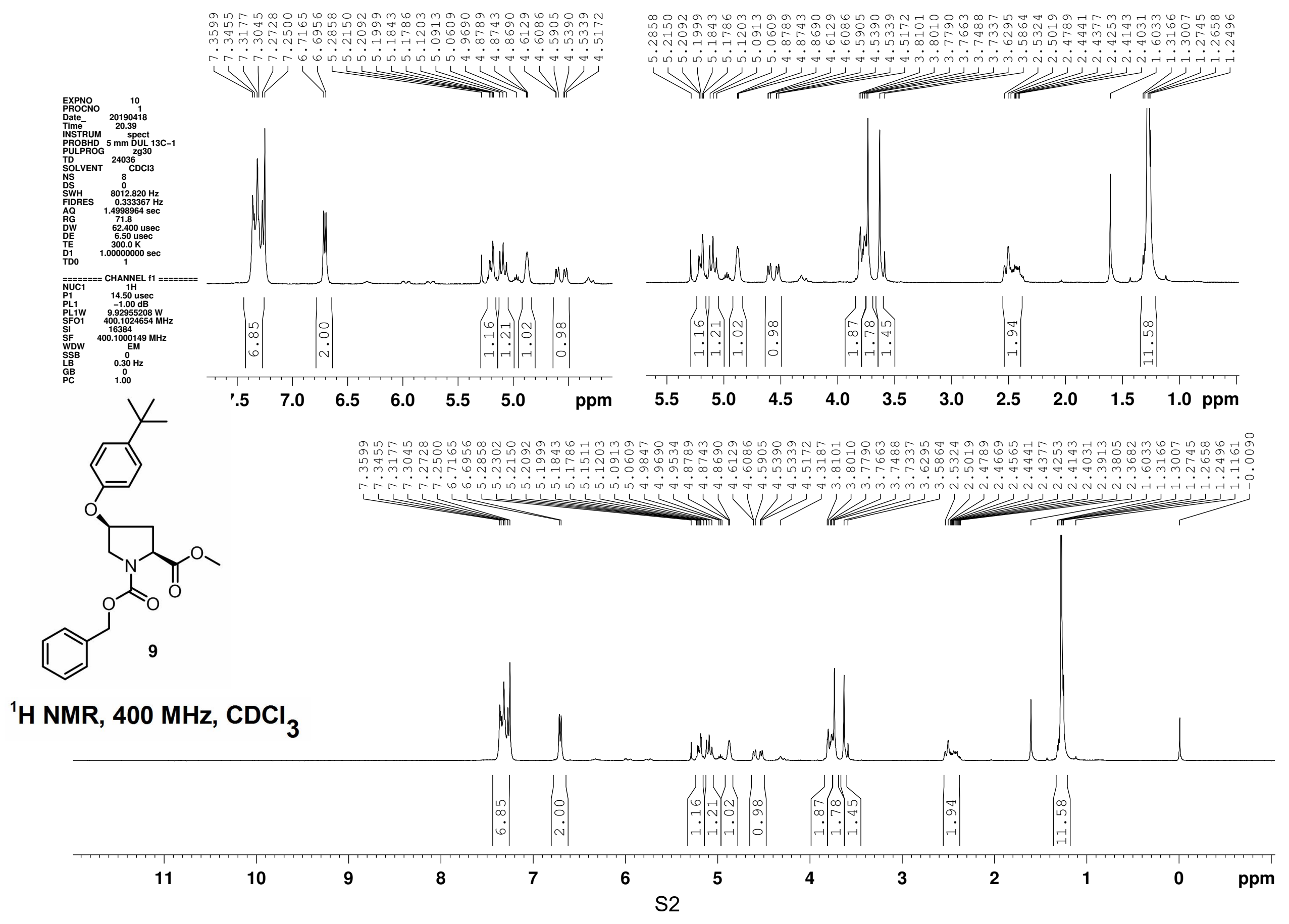




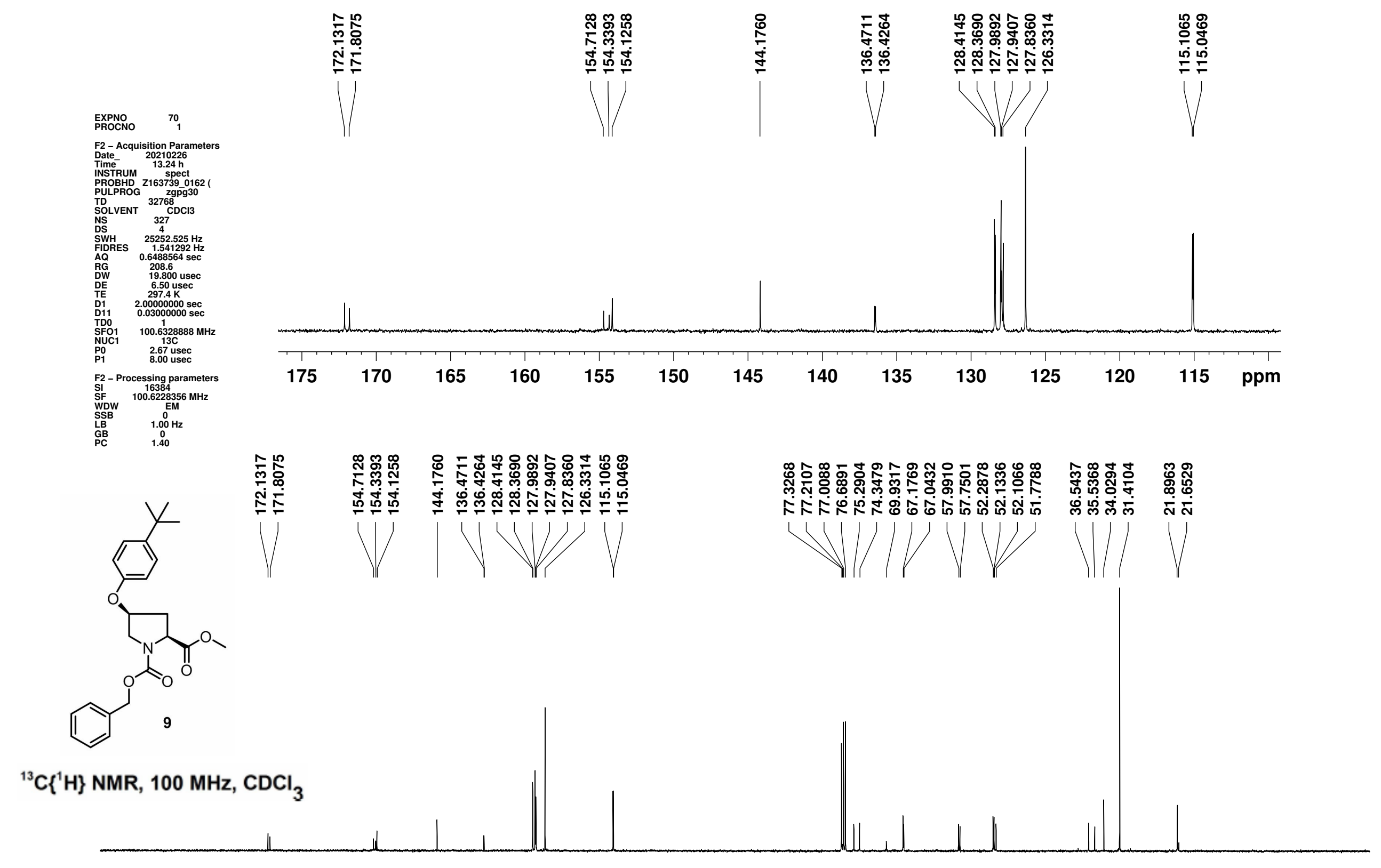

$\begin{array}{llllllllllllllllllll}190 & 180 & 170 & 160 & 150 & 140 & 130 & 120 & 110 & 100 & 90 & 80 & 70 & 60 & 50 & 40 & 30 & 20 & 10 & \mathrm{ppm}\end{array}$ 

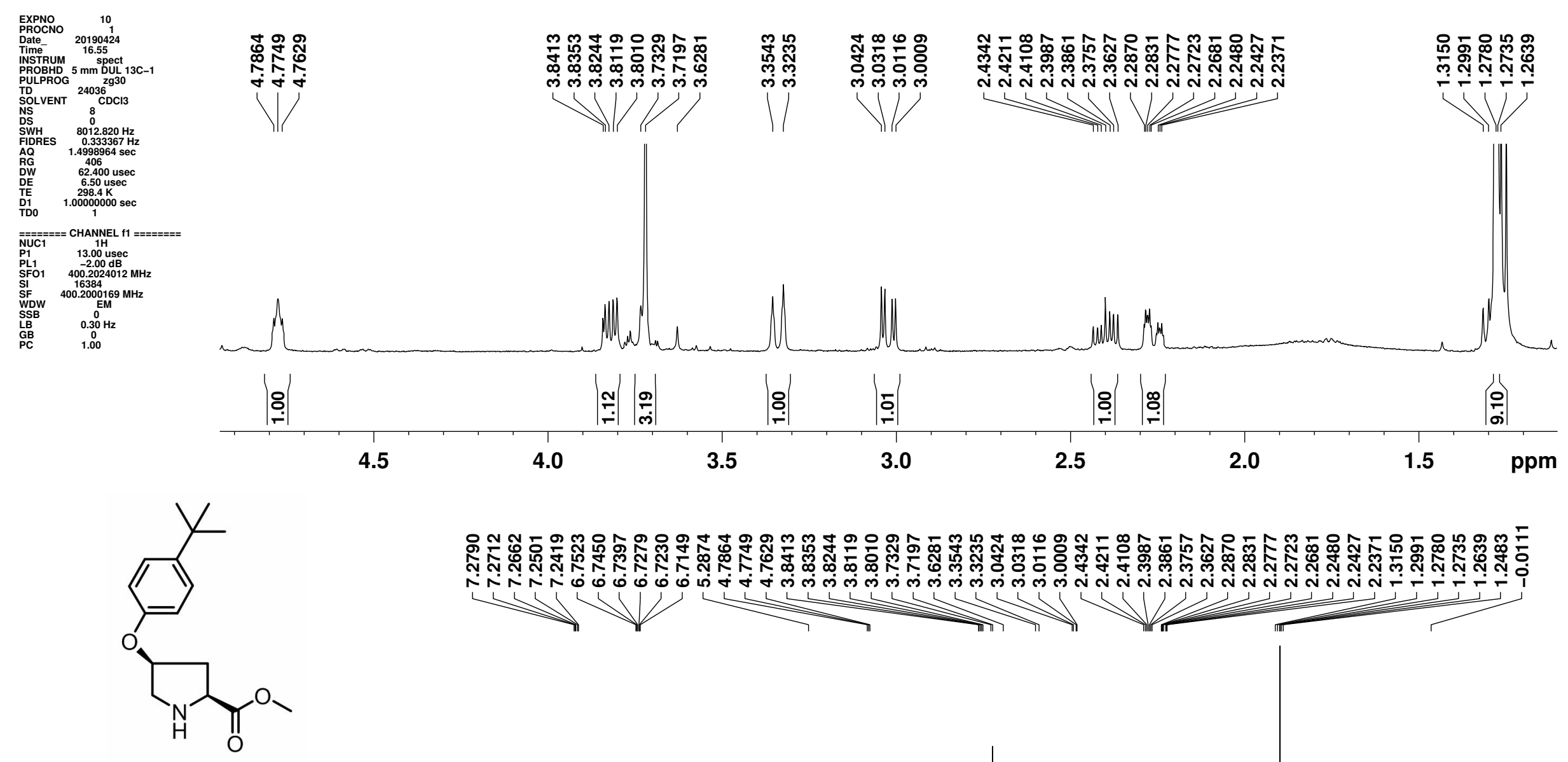

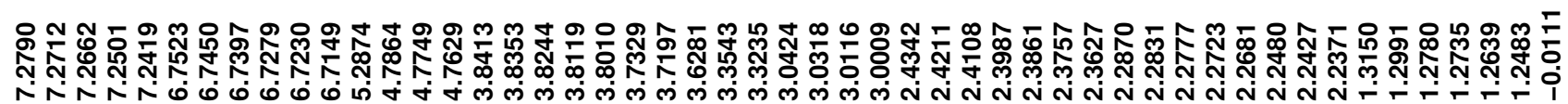
1

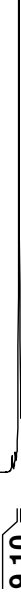

9

8

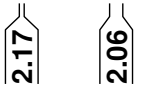

6

5

4

3

2

1

ppm 

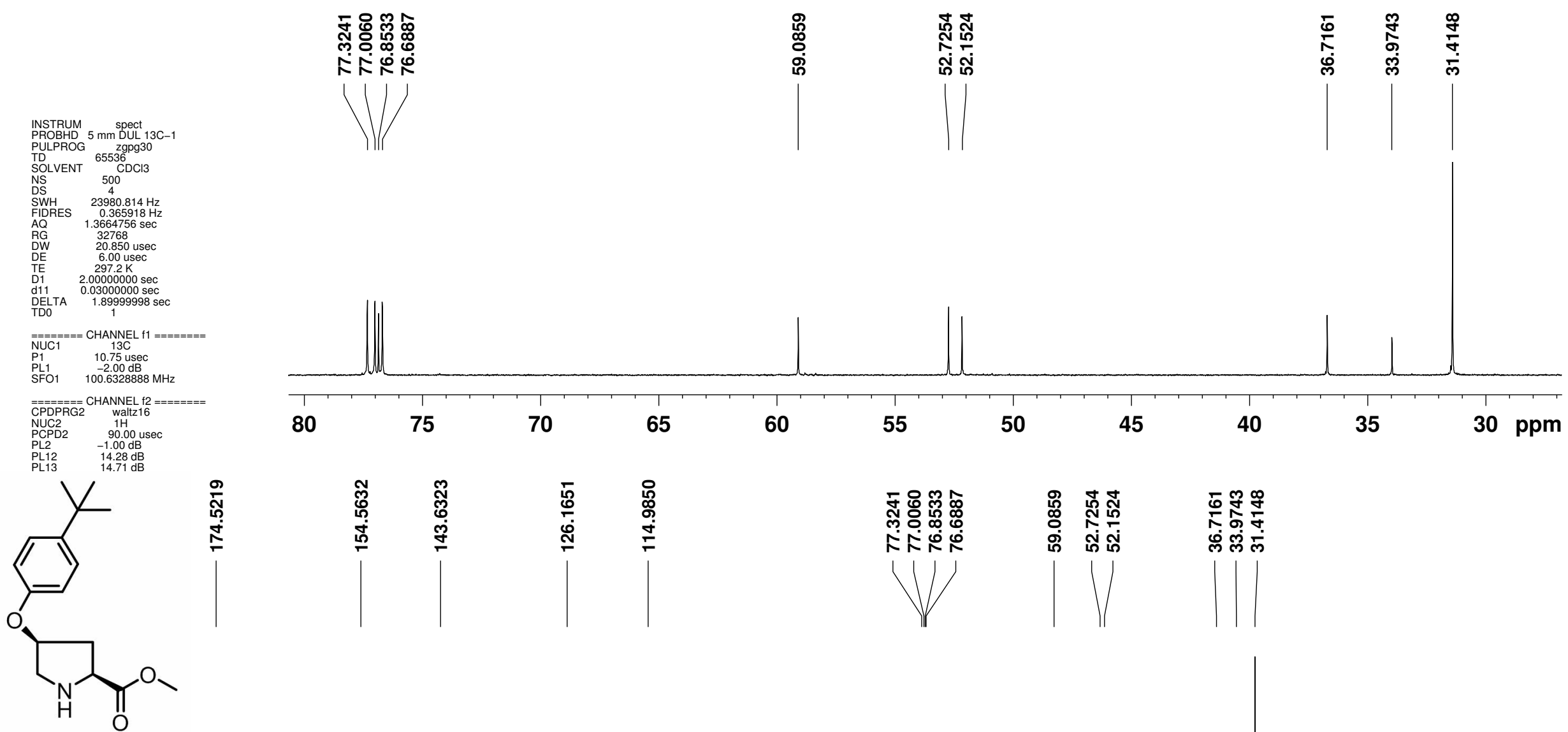

10

${ }^{13} \mathrm{C}\left\{{ }^{1} \mathrm{H}\right\} \mathrm{NMR}, 100 \mathrm{MHz}, \mathrm{CDCl}_{3}$

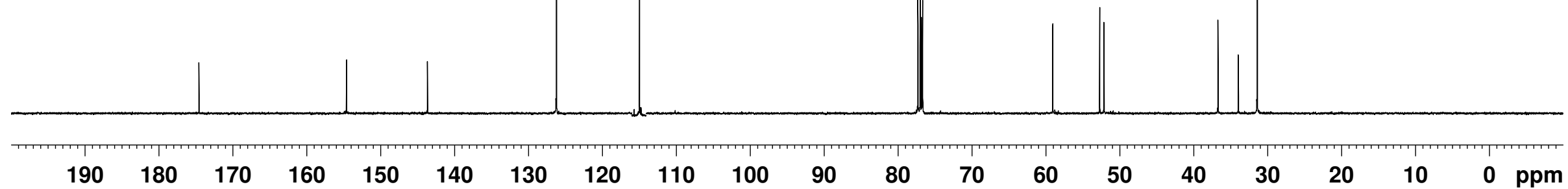



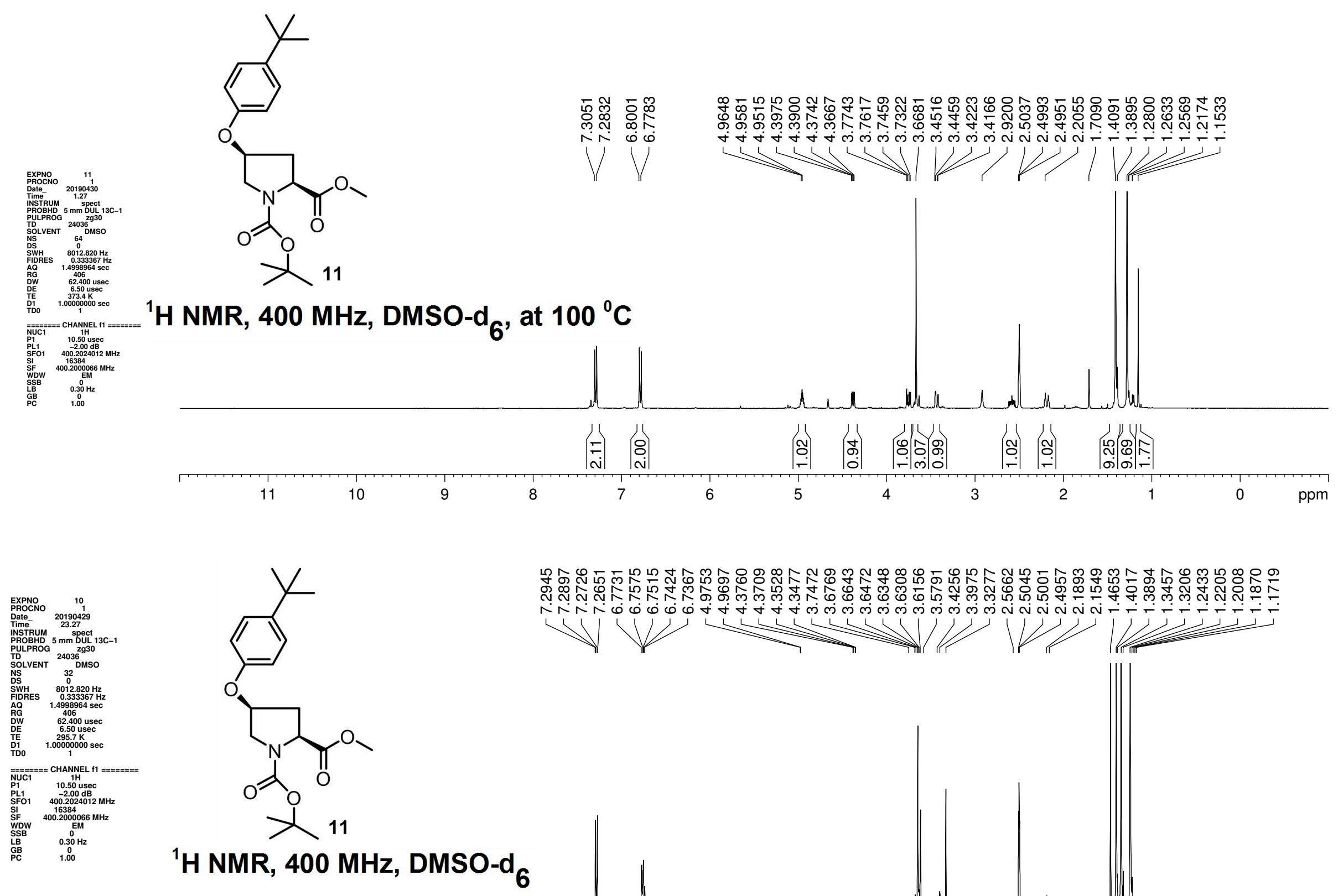

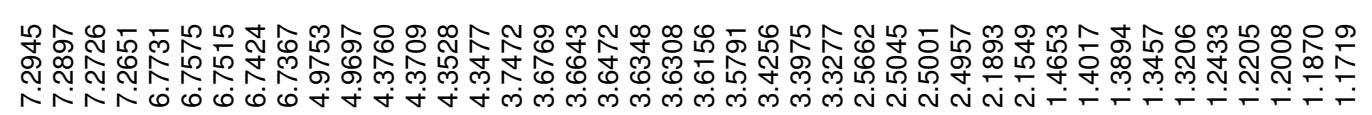

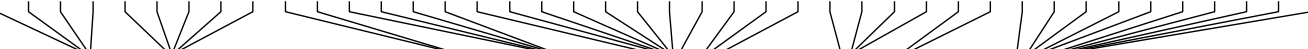

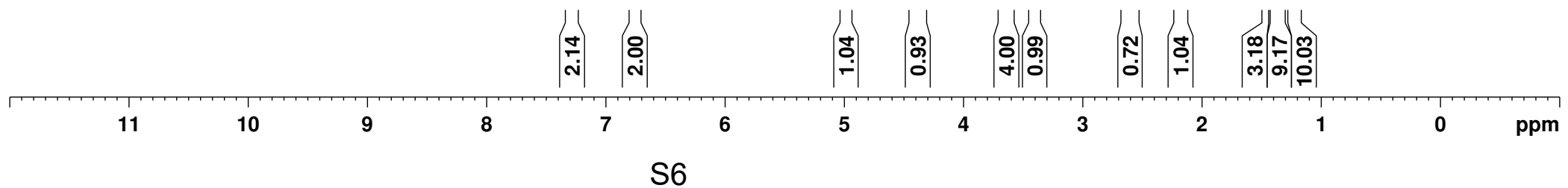



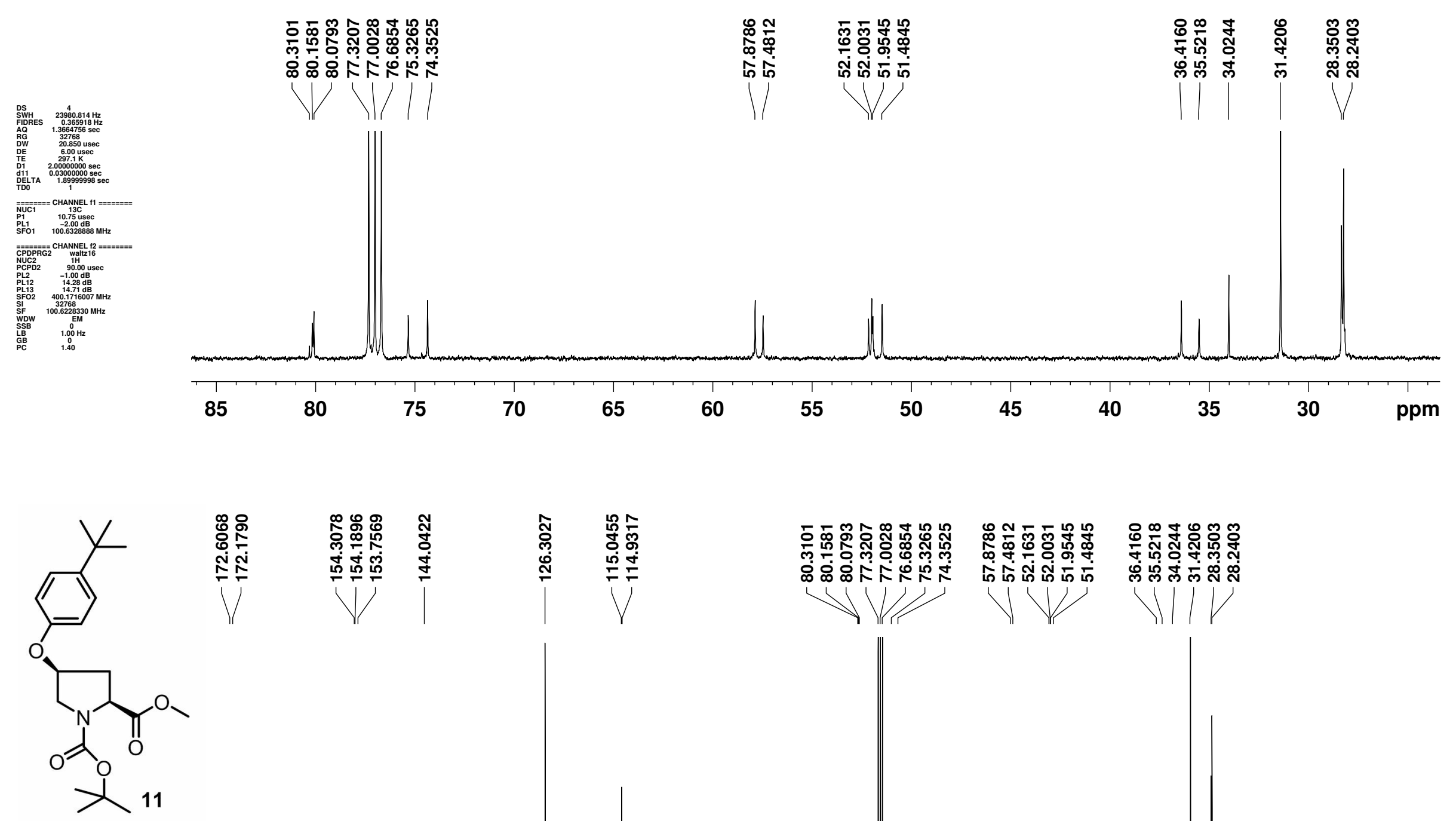

${ }^{13} \mathrm{C}\left\{{ }^{1} \mathrm{H}\right\} \mathrm{NMR}, 100 \mathrm{MHz}, \mathrm{CDCl}_{3}$
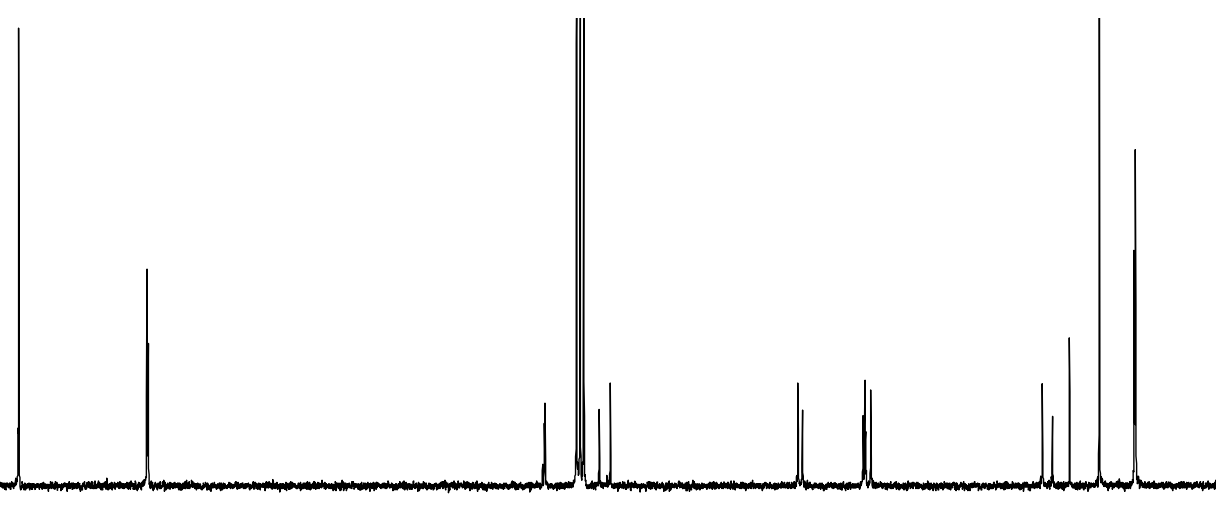

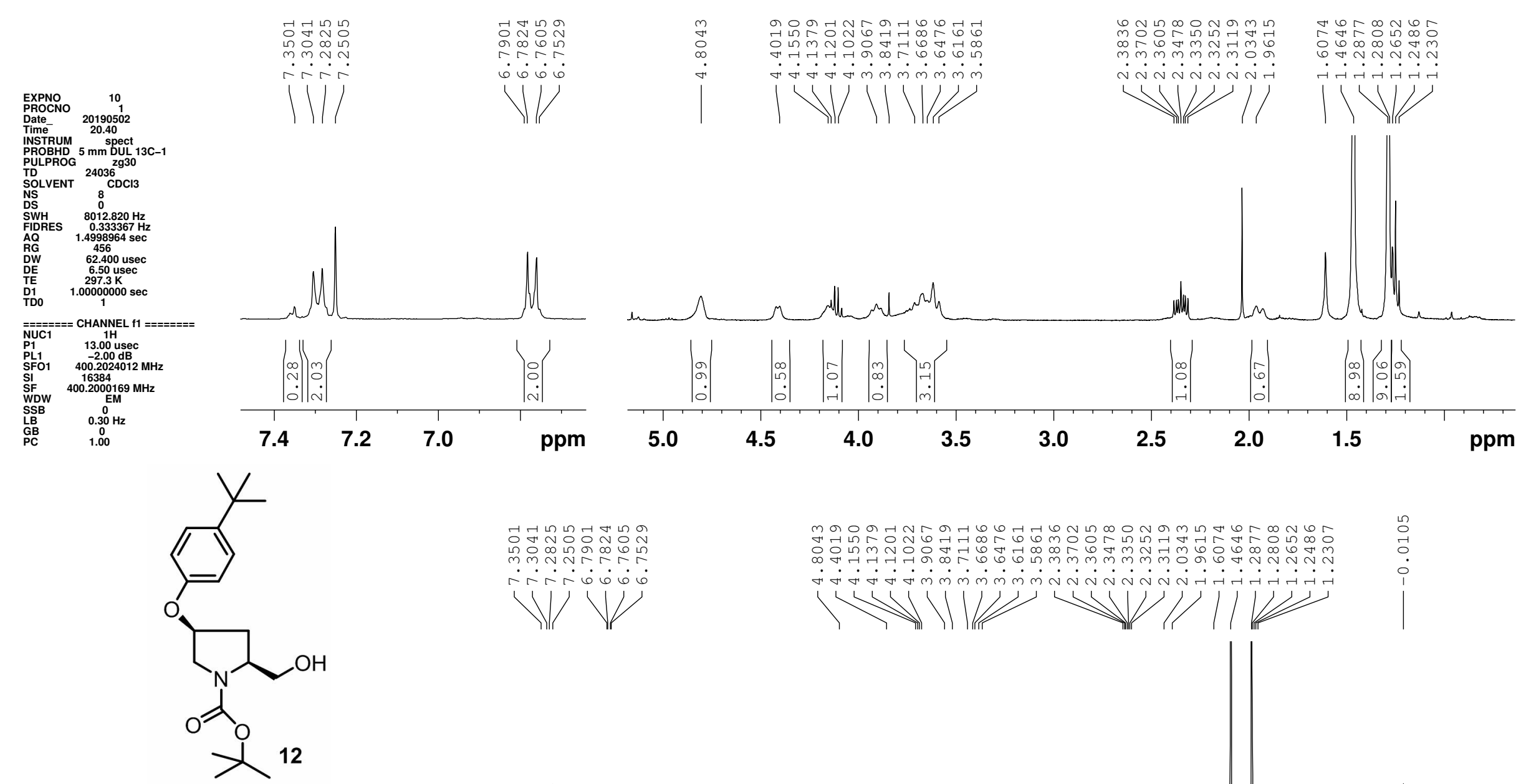

${ }^{1} \mathrm{H}$ NMR, $400 \mathrm{MHz}, \mathrm{CDCl}_{3}$

11

10

9

8

7

6 

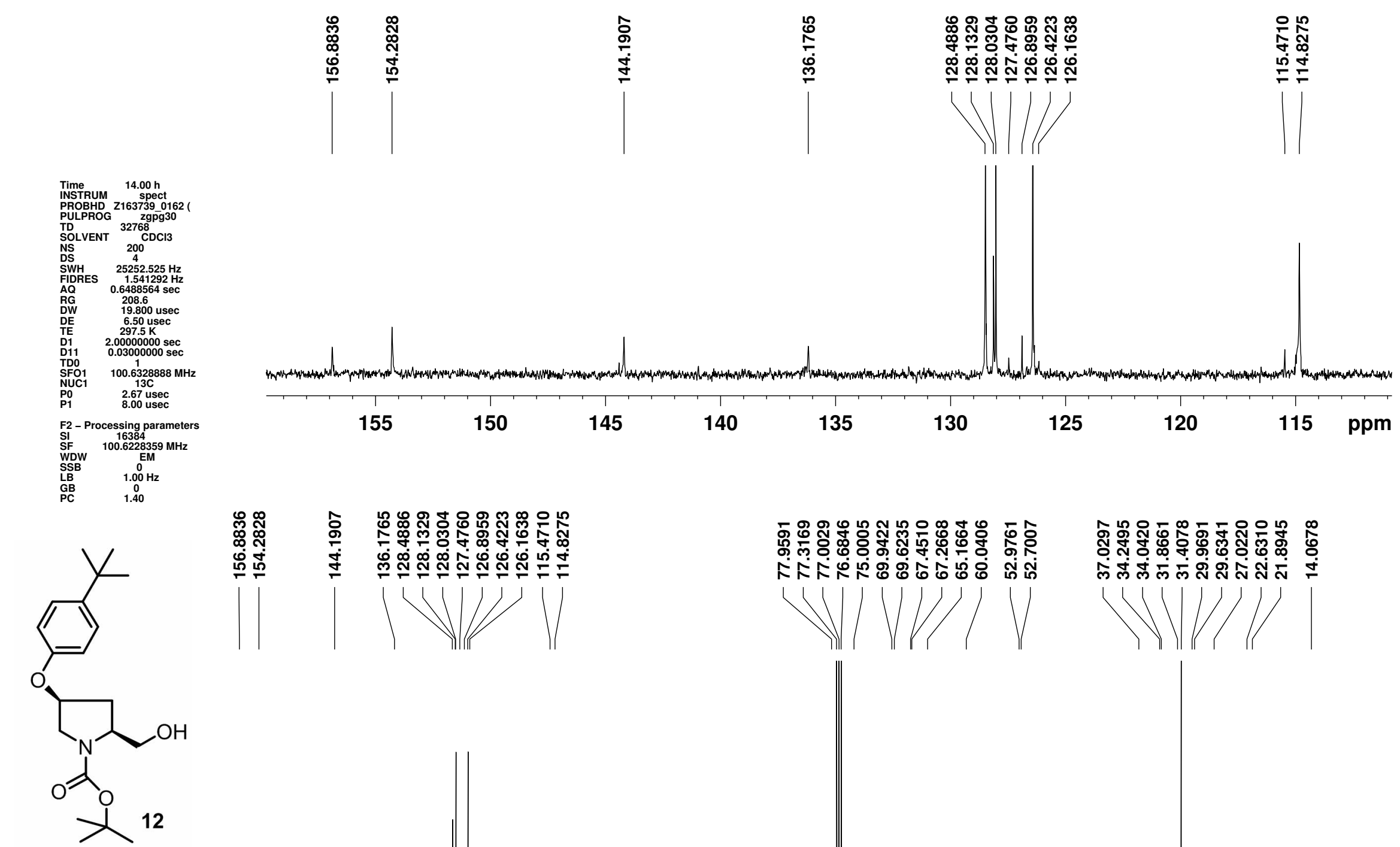

${ }^{13} \mathrm{C}\left\{{ }^{1} \mathrm{H}\right\} \mathrm{NMR}, 100 \mathrm{MHz}, \mathrm{CDCl}_{3}$

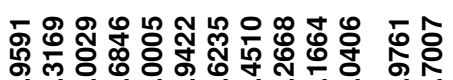
र下ト

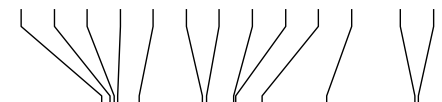

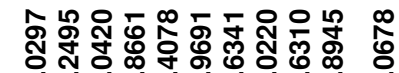

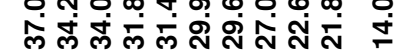

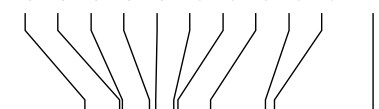

130

120

110100

90

80

70

60

50

40

30

20 

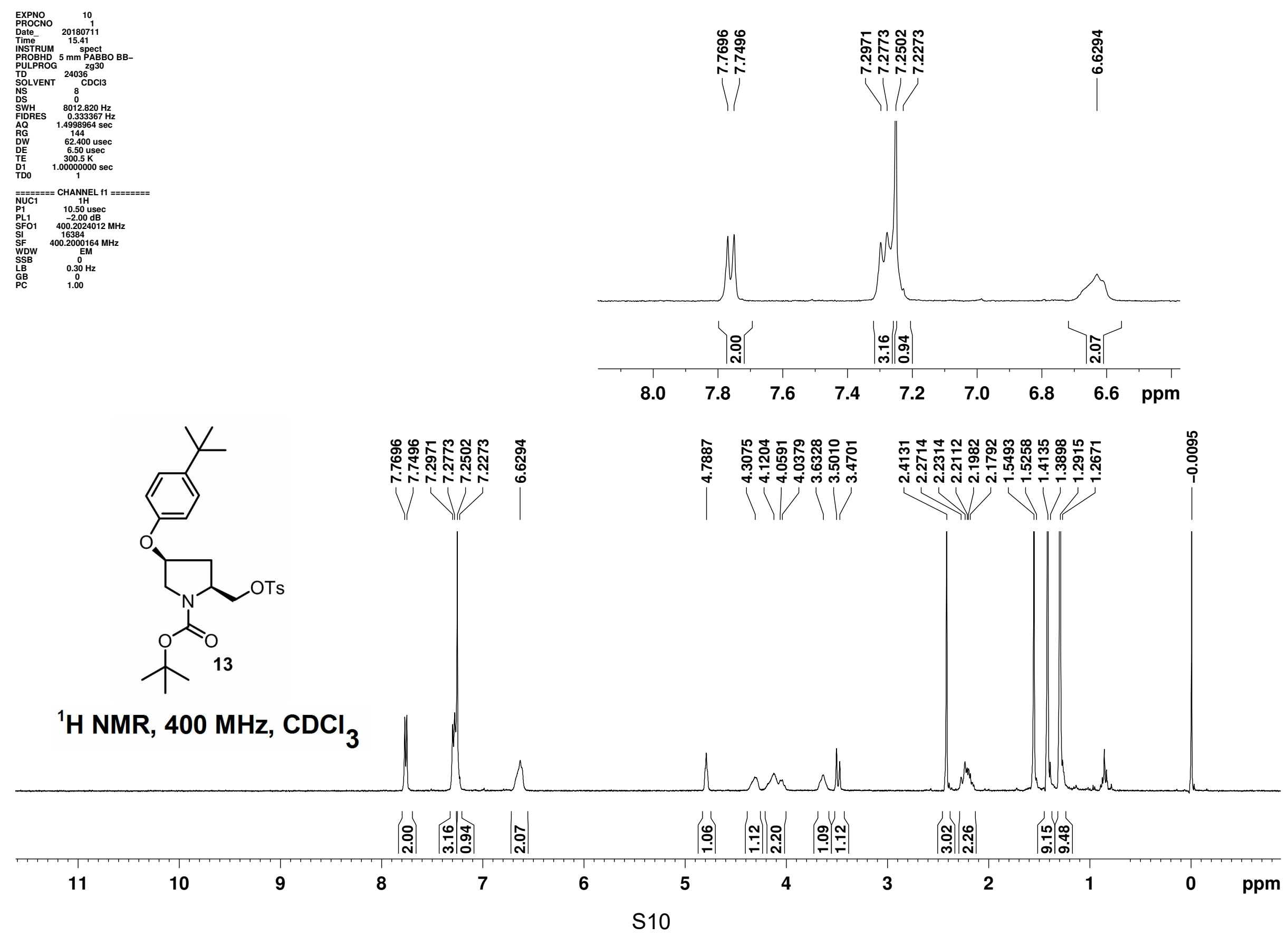

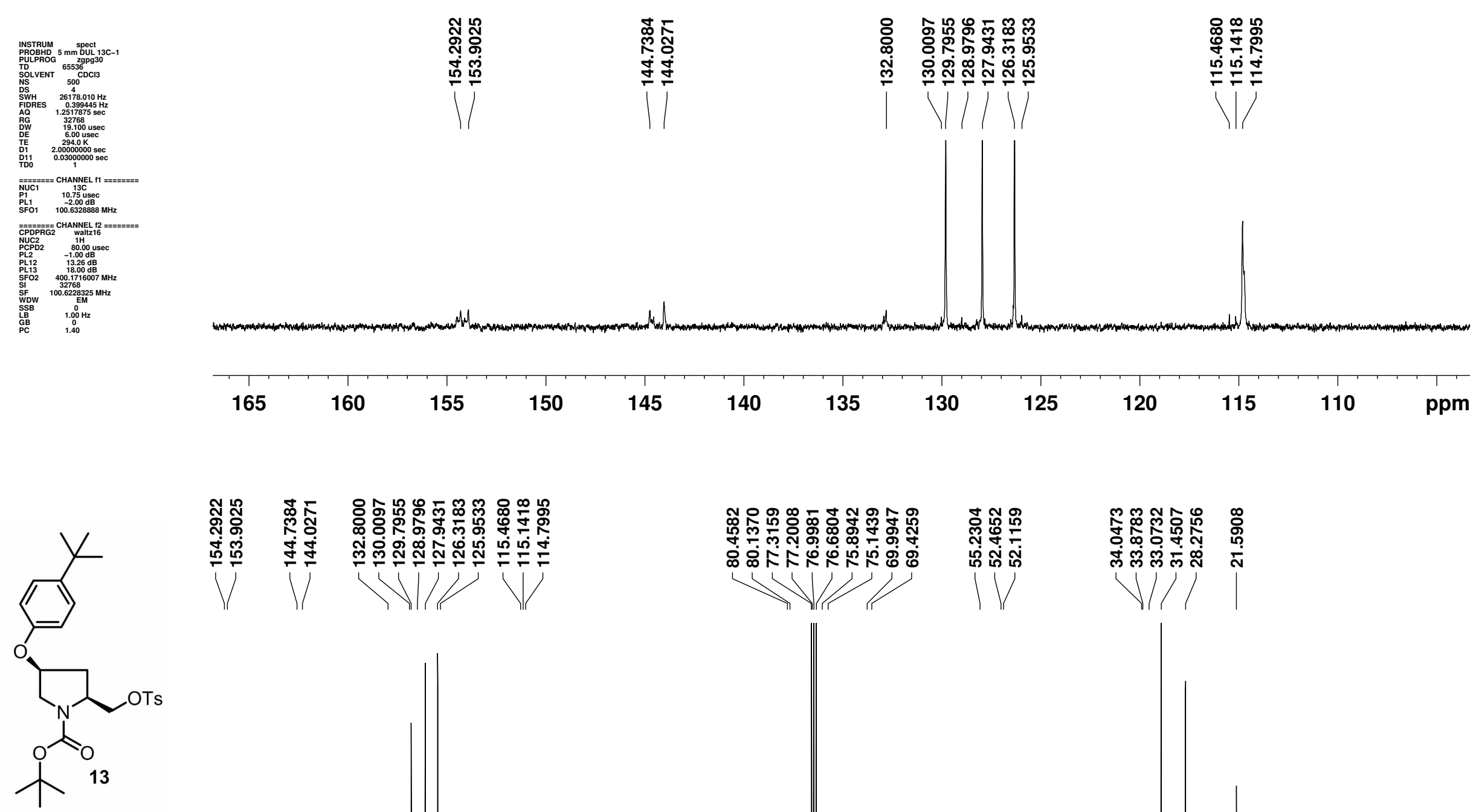

${ }^{13} \mathrm{C}\left\{{ }^{1} \mathrm{H}\right\} \mathrm{NMR}, 100 \mathrm{MHz}, \mathrm{CDCl}_{3}$
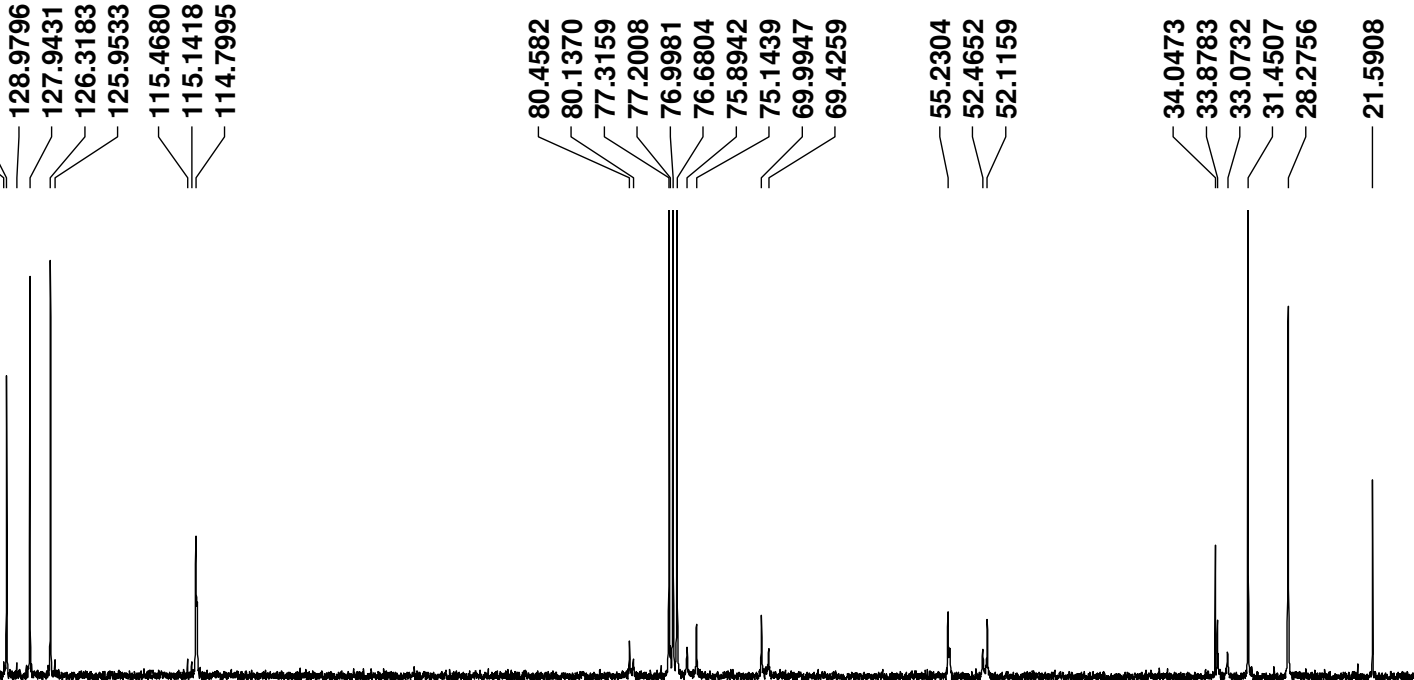

170

160

150

140

130

120

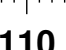

100

90

80

70

60

50

40

30

20

100 ppm 

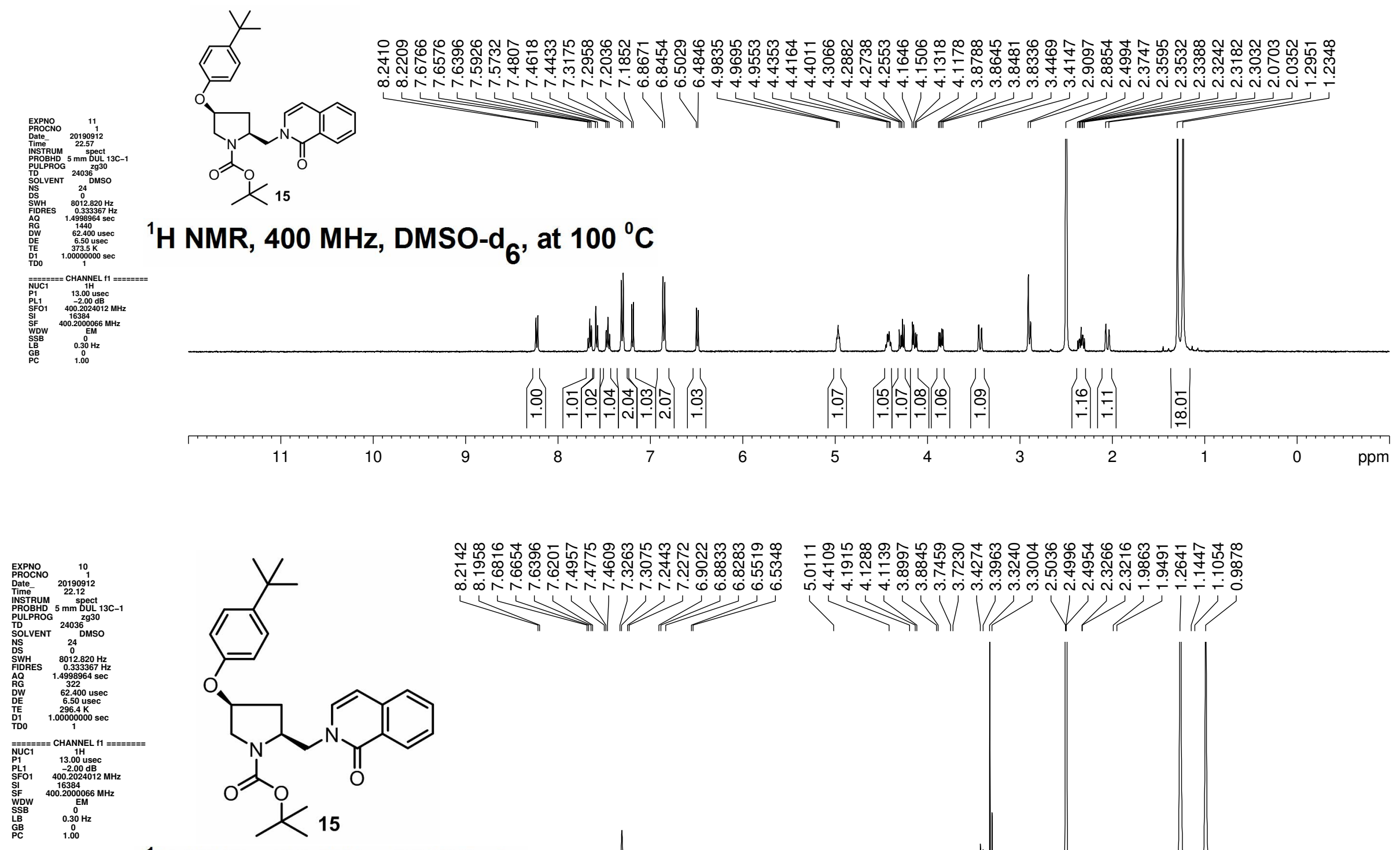

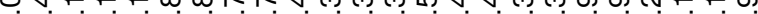

1

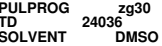

NS 24 MDSO

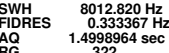

$2.50 .04 \mathrm{usec}$
1.00000000

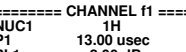

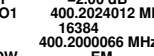

1.00

${ }^{1} \mathrm{H}$ NMR, $400 \mathrm{MHz}$, DMSO-d 6

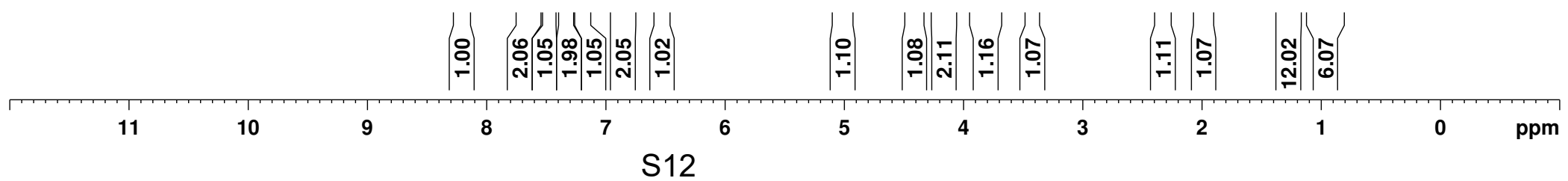


TCGLS/ARD/NMR02/K02
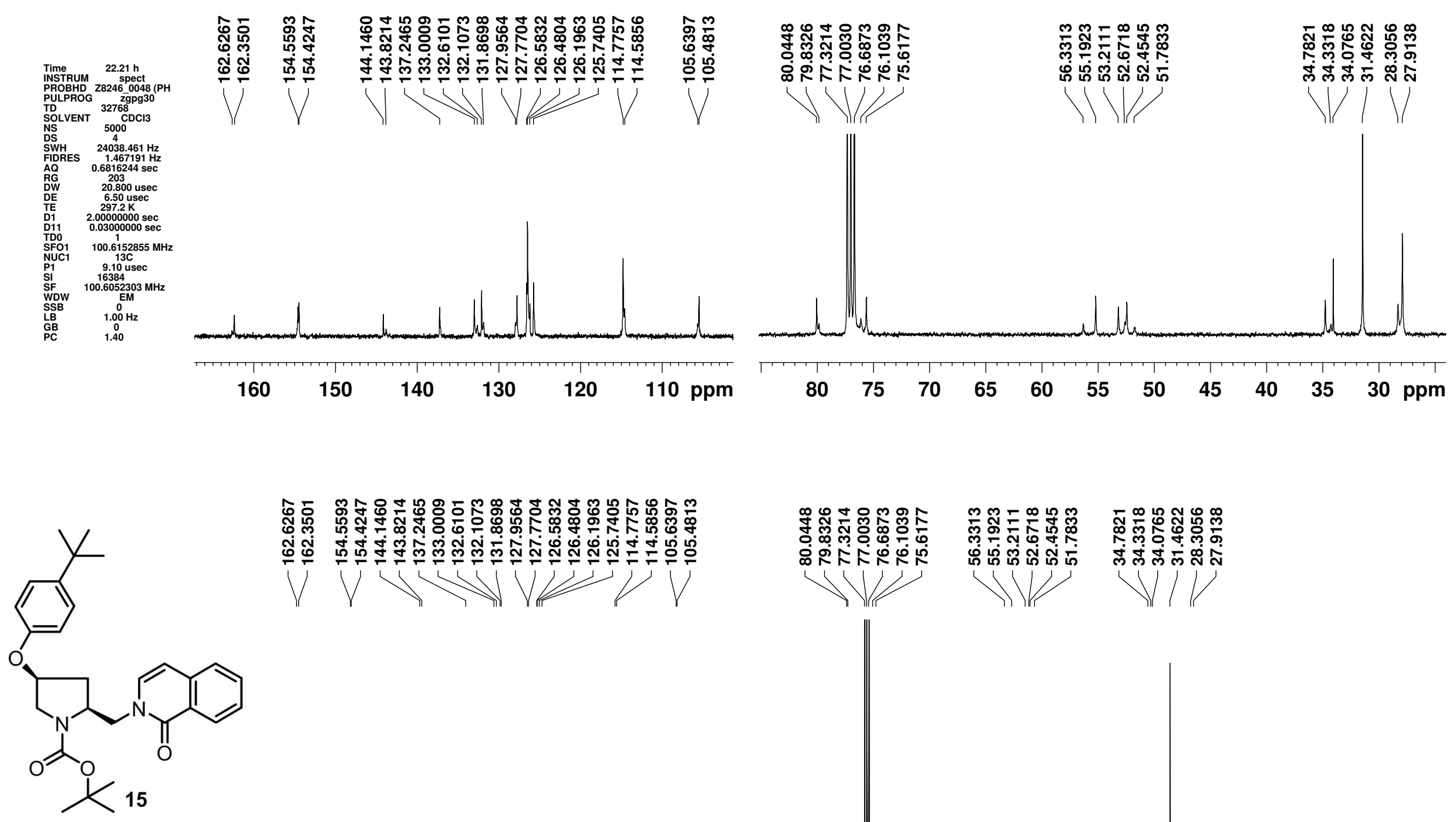

${ }^{13} \mathrm{C}\left\{{ }^{1} \mathrm{H}\right\} \mathrm{NMR}, 100 \mathrm{MHz}, \mathrm{CDCl}_{3}$

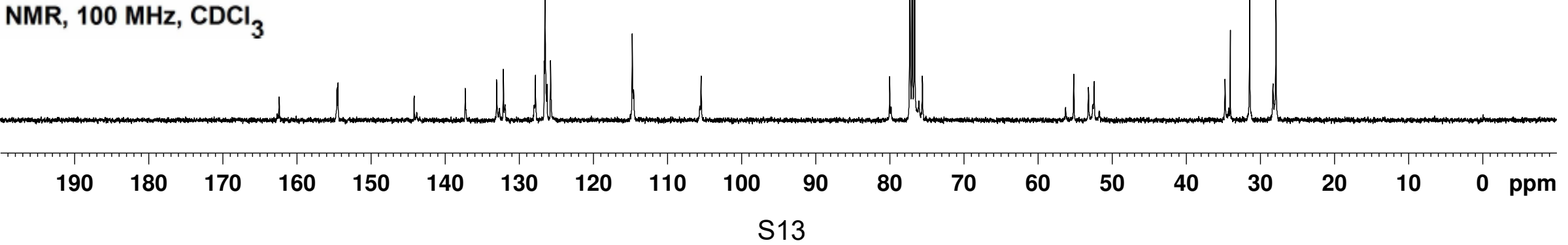



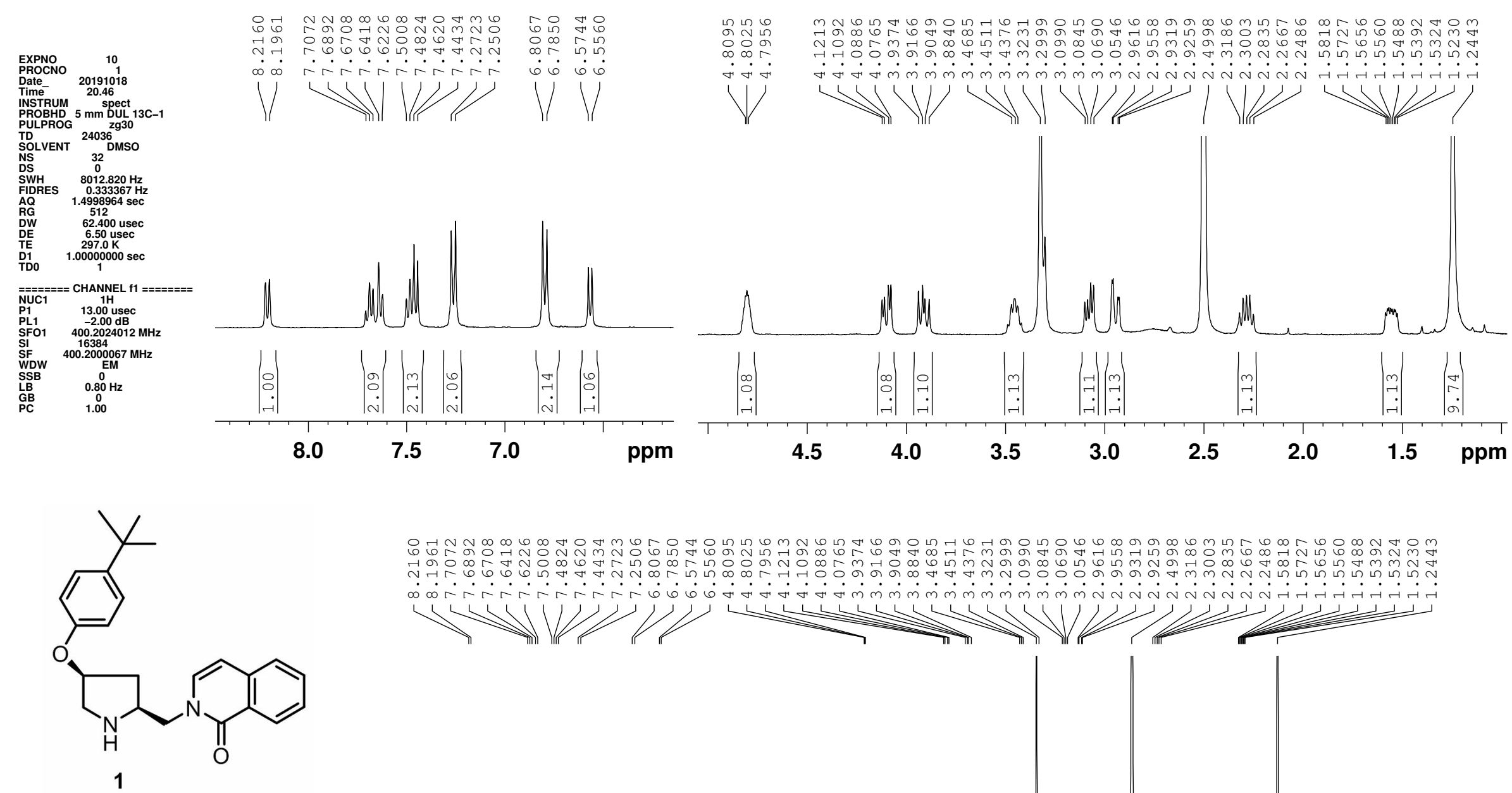

${ }^{1} \mathrm{H}_{\mathrm{NMR}}, 400 \mathrm{MHz}$, DMSO-d 6

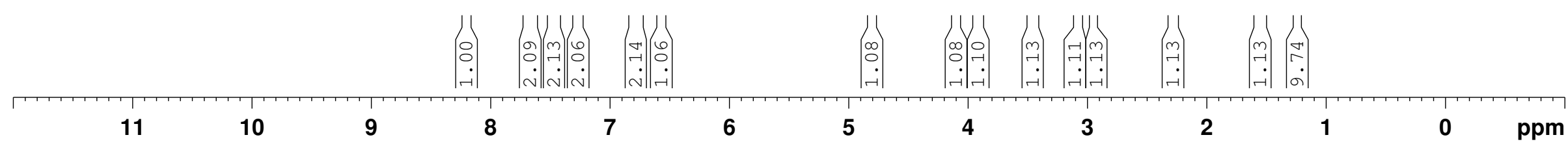



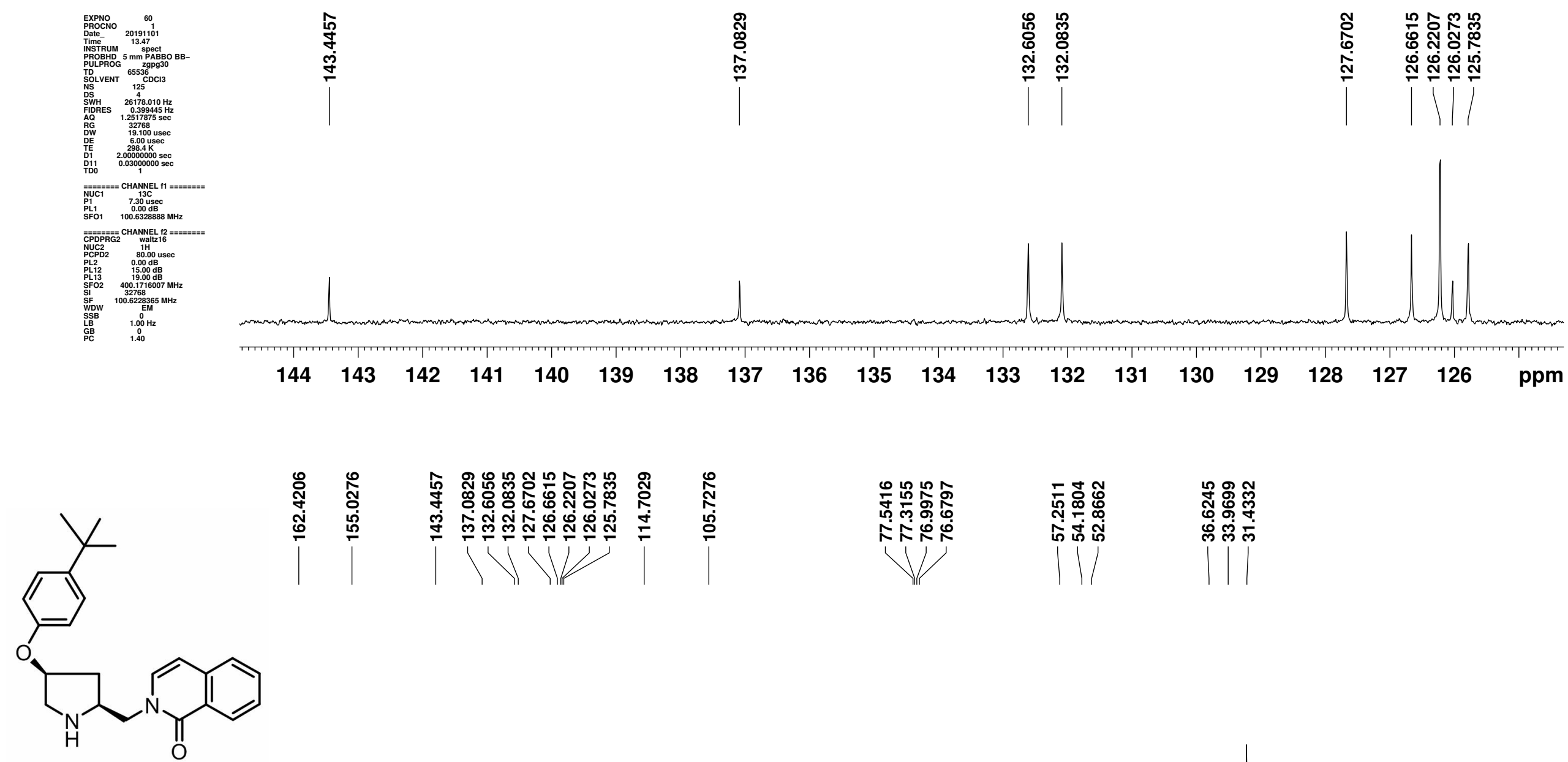

1

${ }^{13} \mathrm{C}\left\{{ }^{1} \mathrm{H}\right\} \mathrm{NMR}, 100 \mathrm{MHz}, \mathrm{CDCl}_{3}$

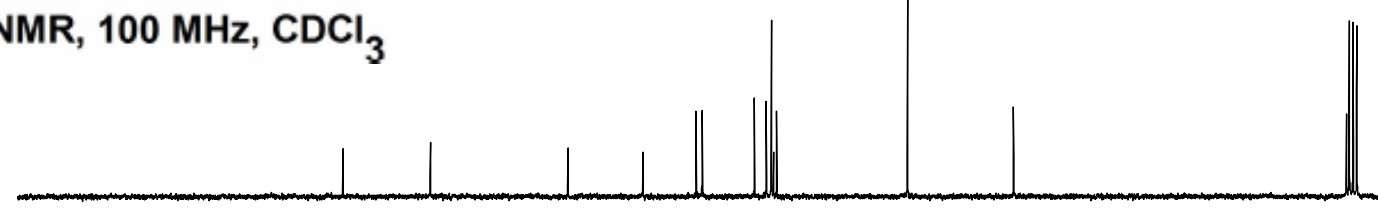




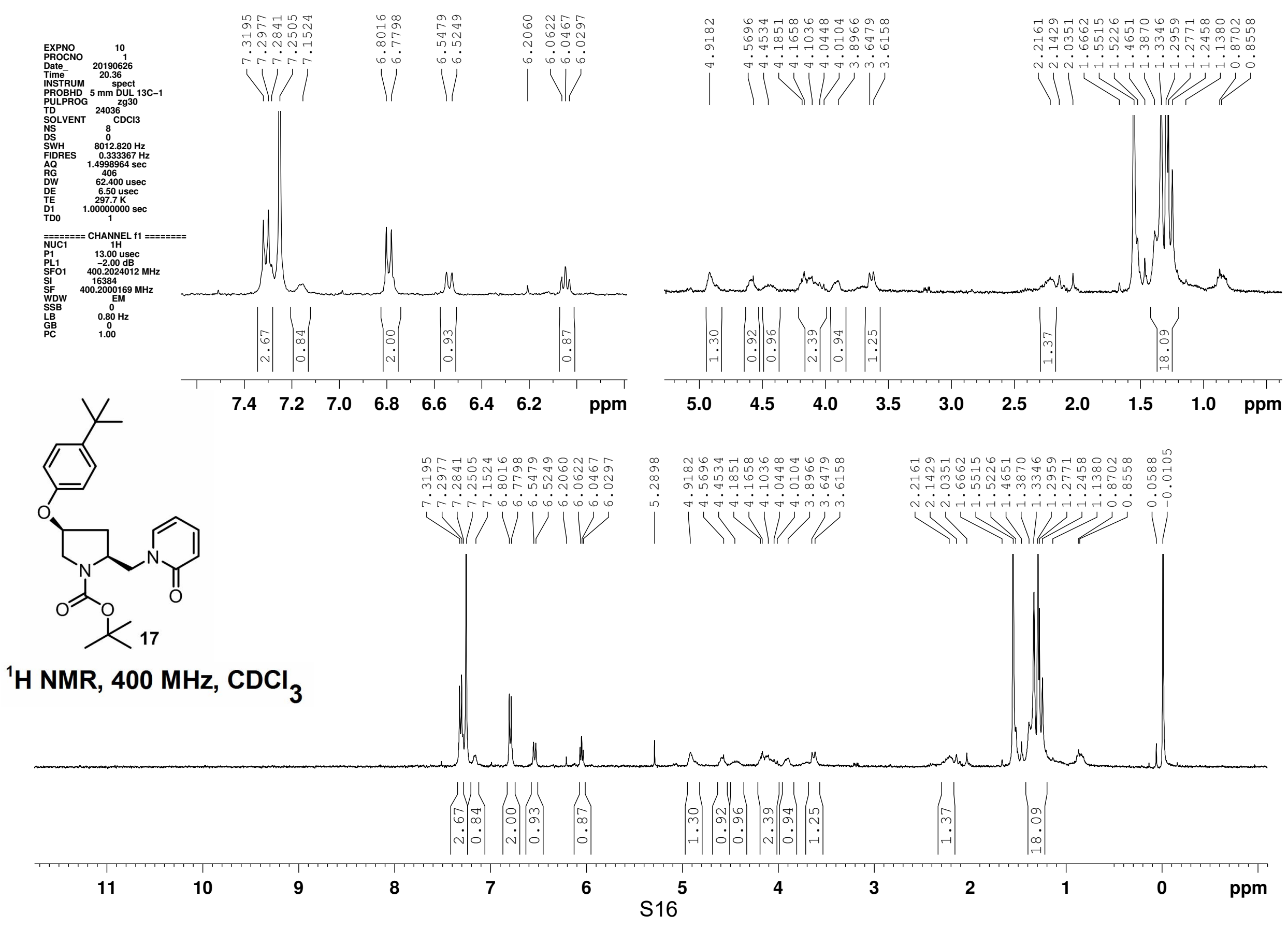



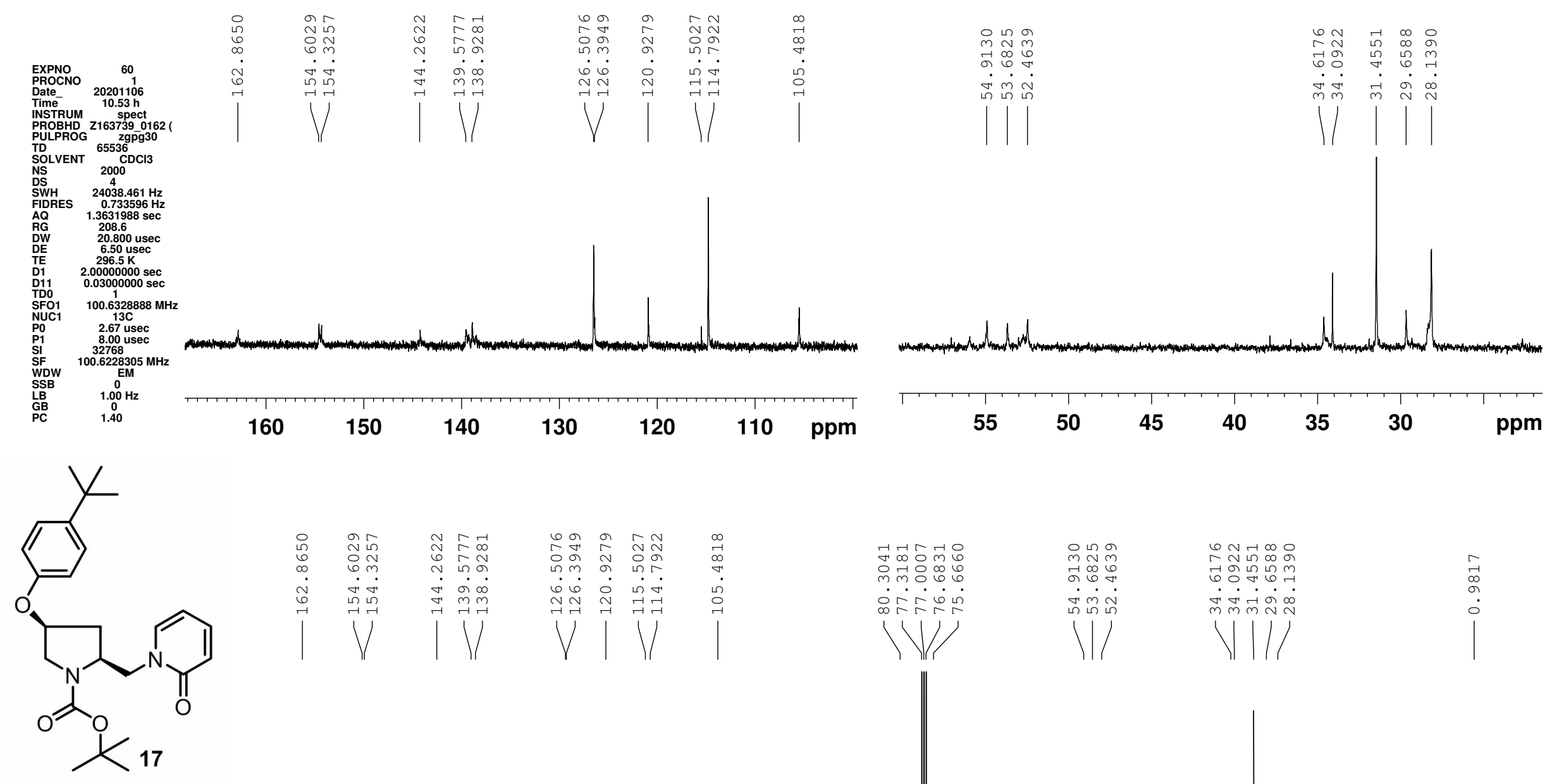

${ }^{13} \mathrm{C}\left\{{ }^{1} \mathrm{H}\right\} \mathrm{NMR}, 100 \mathrm{MHz}, \mathrm{CDCl}_{3}$

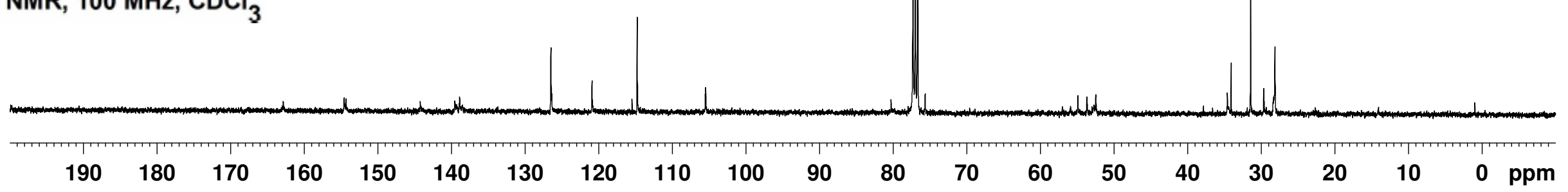




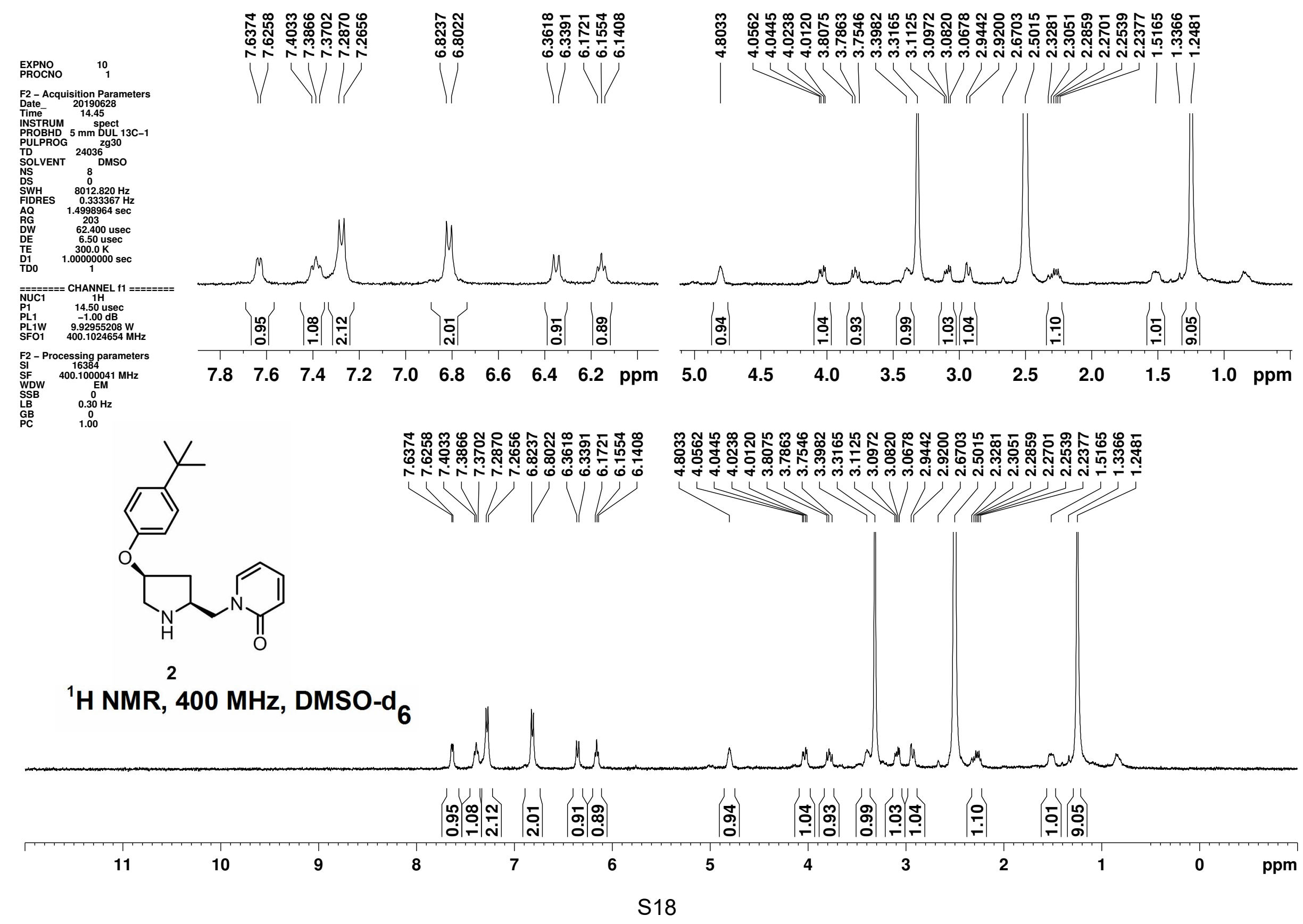




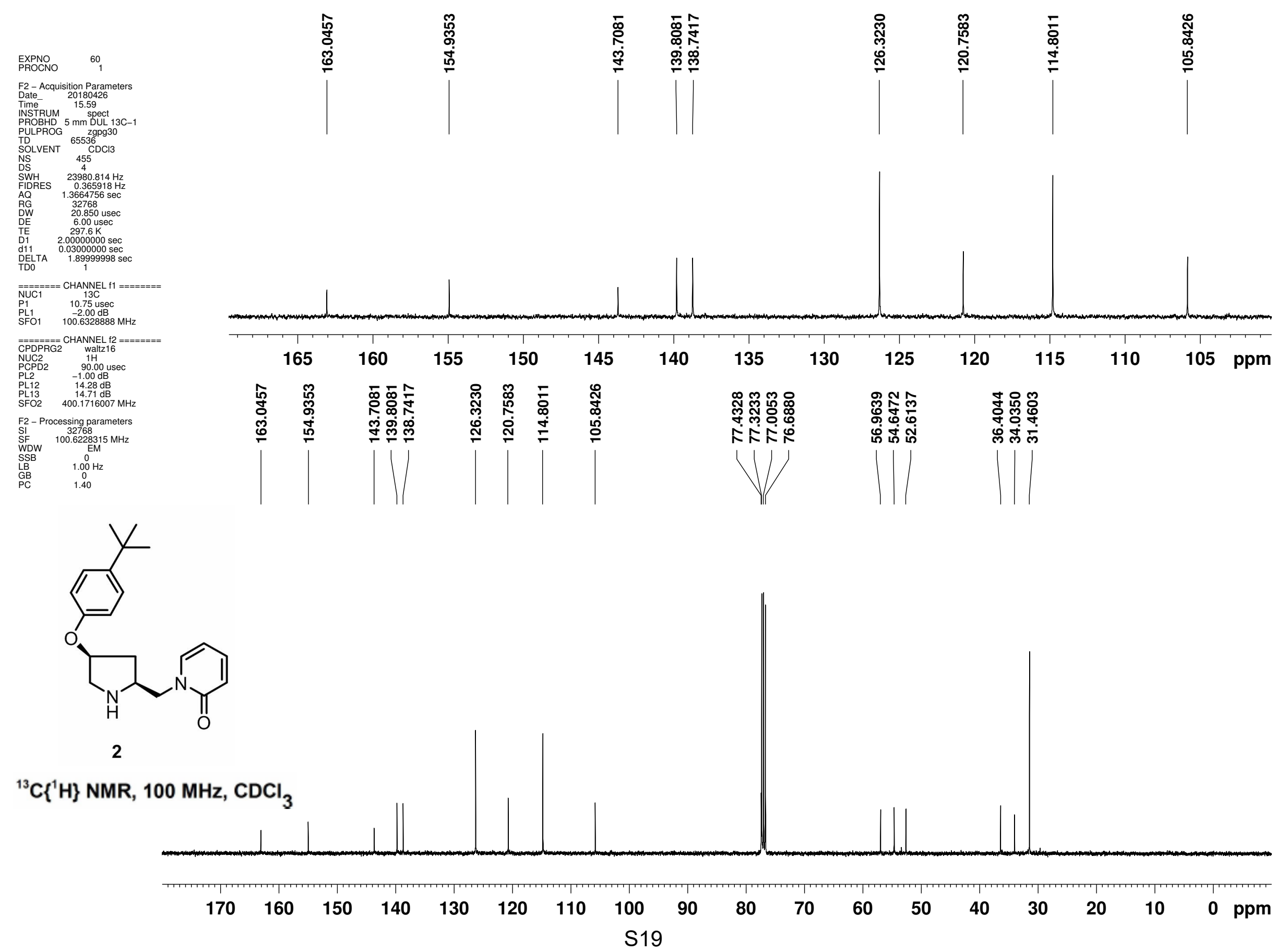



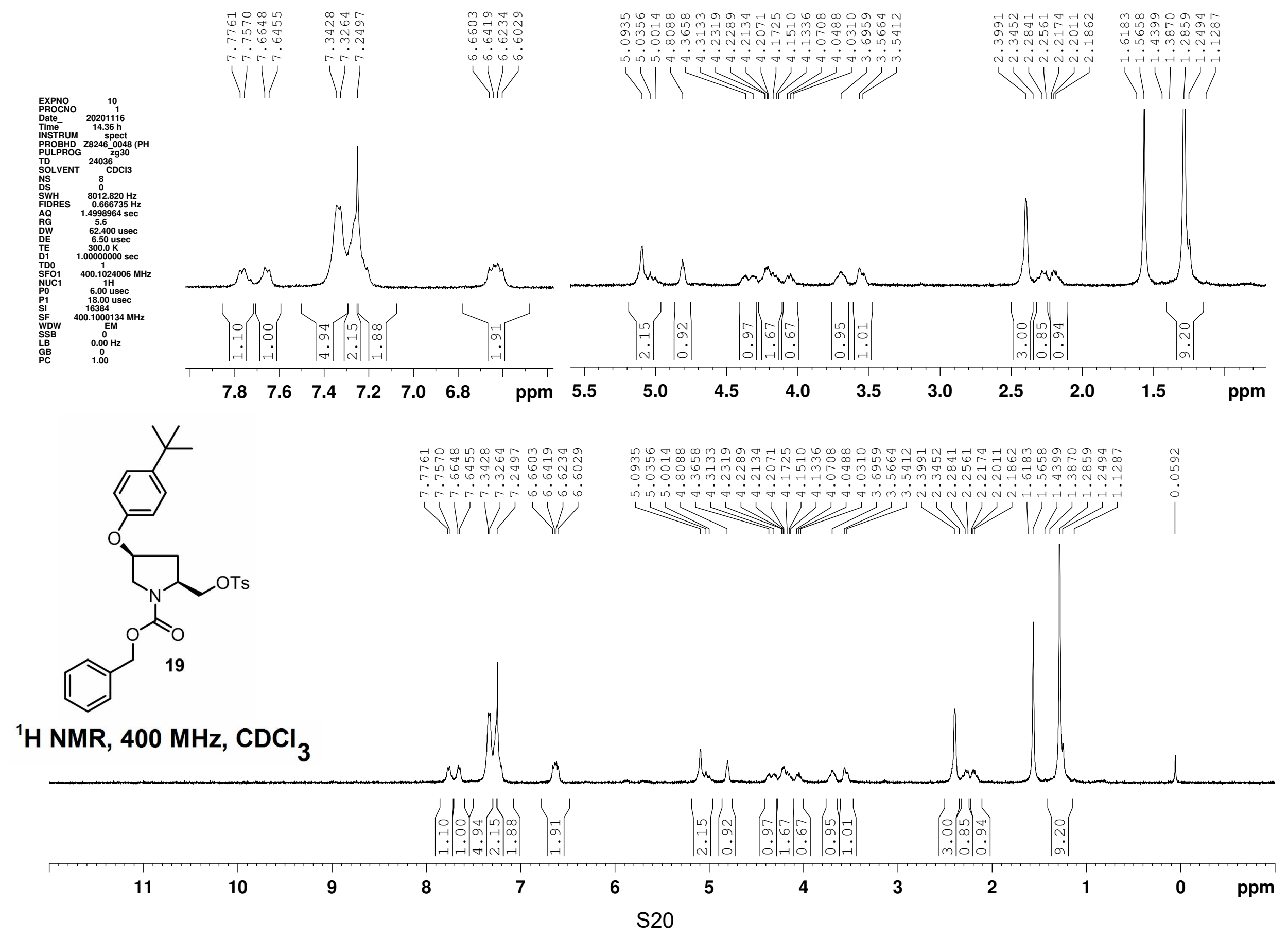

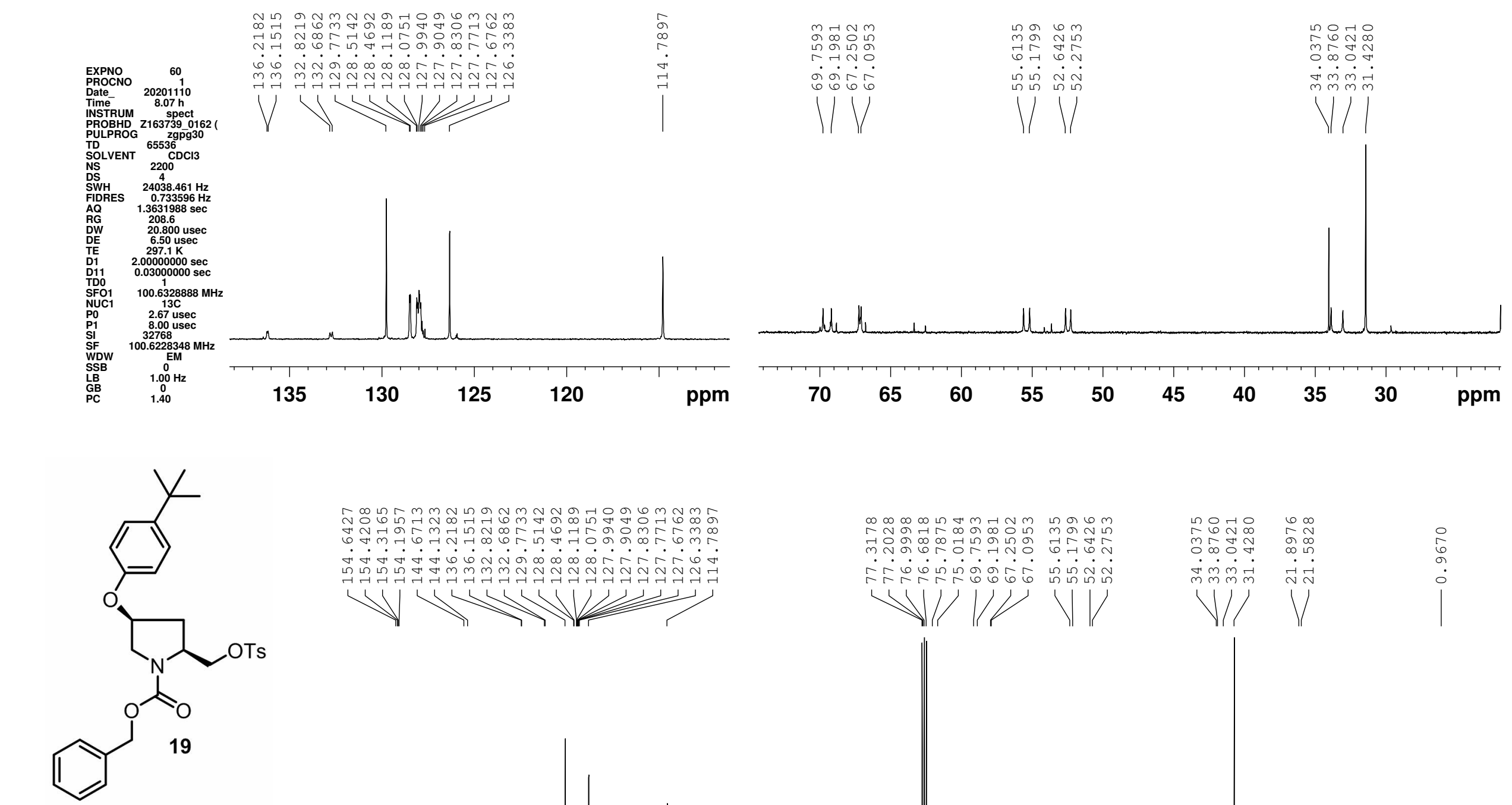

${ }^{13} \mathrm{C}\left\{{ }^{1} \mathrm{H}\right\} \mathrm{NMR}, 100 \mathrm{MHz}, \mathrm{CDCl}_{3}$
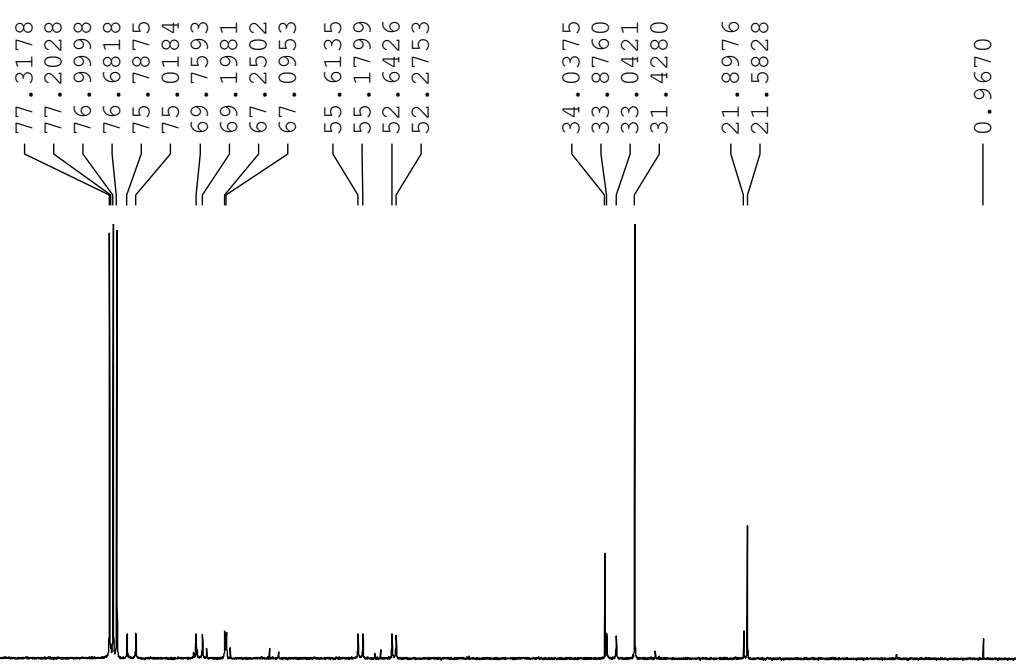

190

180

170

160

150

140

130

120

110100

90

80

70

$60 \quad 50$

5040 

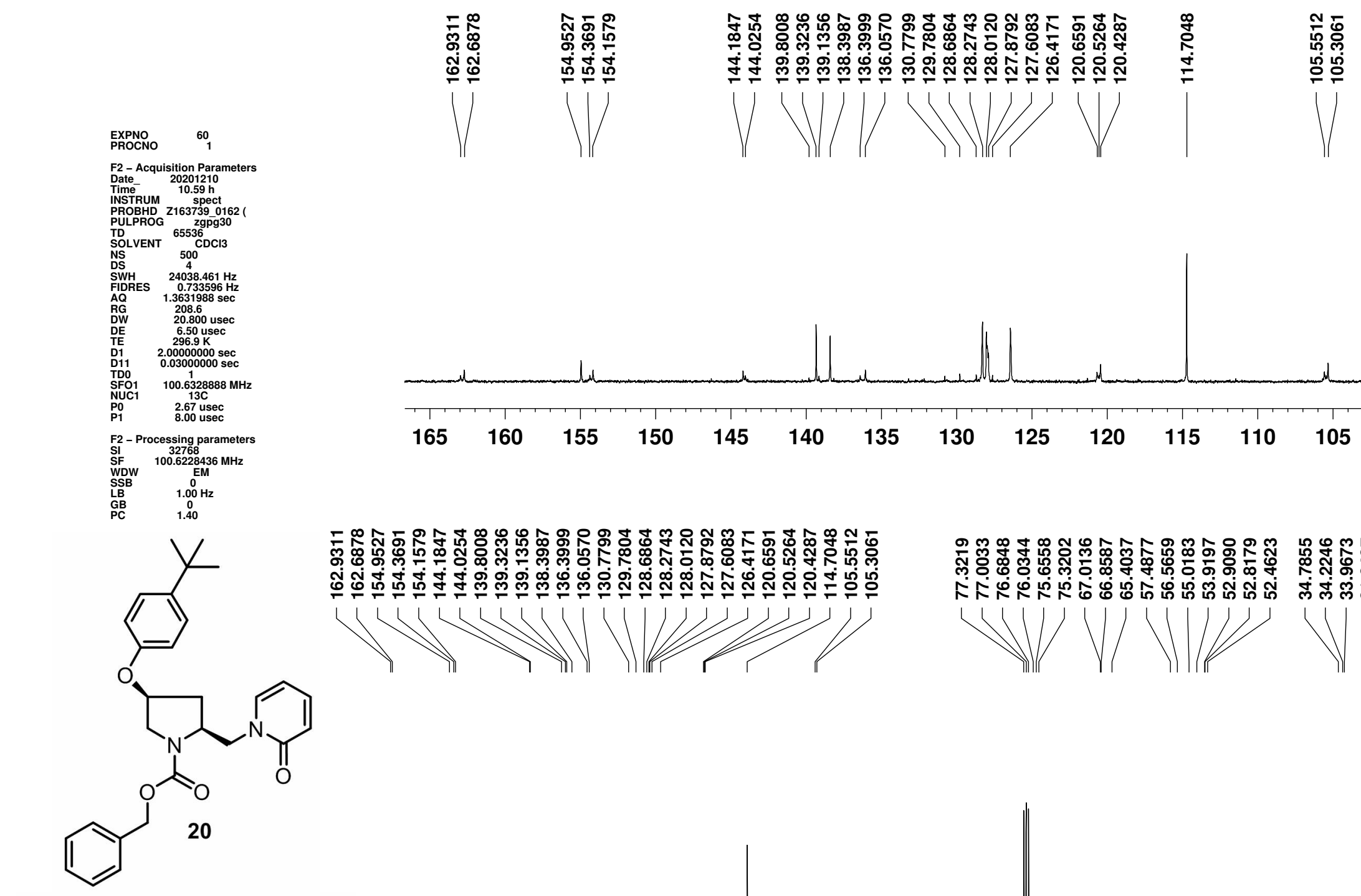

${ }^{13} \mathrm{C}\left\{{ }^{1} \mathrm{H}\right\} \mathrm{NMR}, 100 \mathrm{MHz}, \mathrm{CDCl}_{3}$

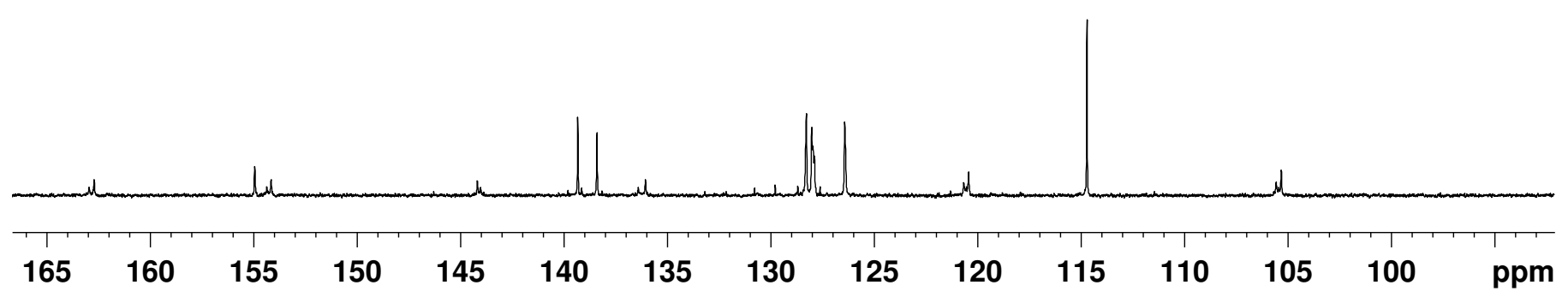

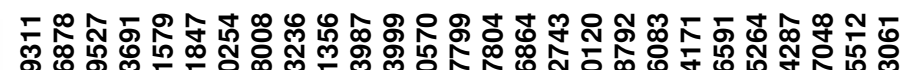

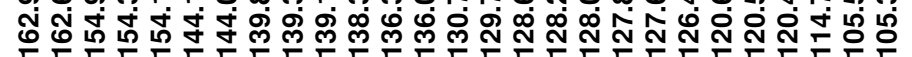
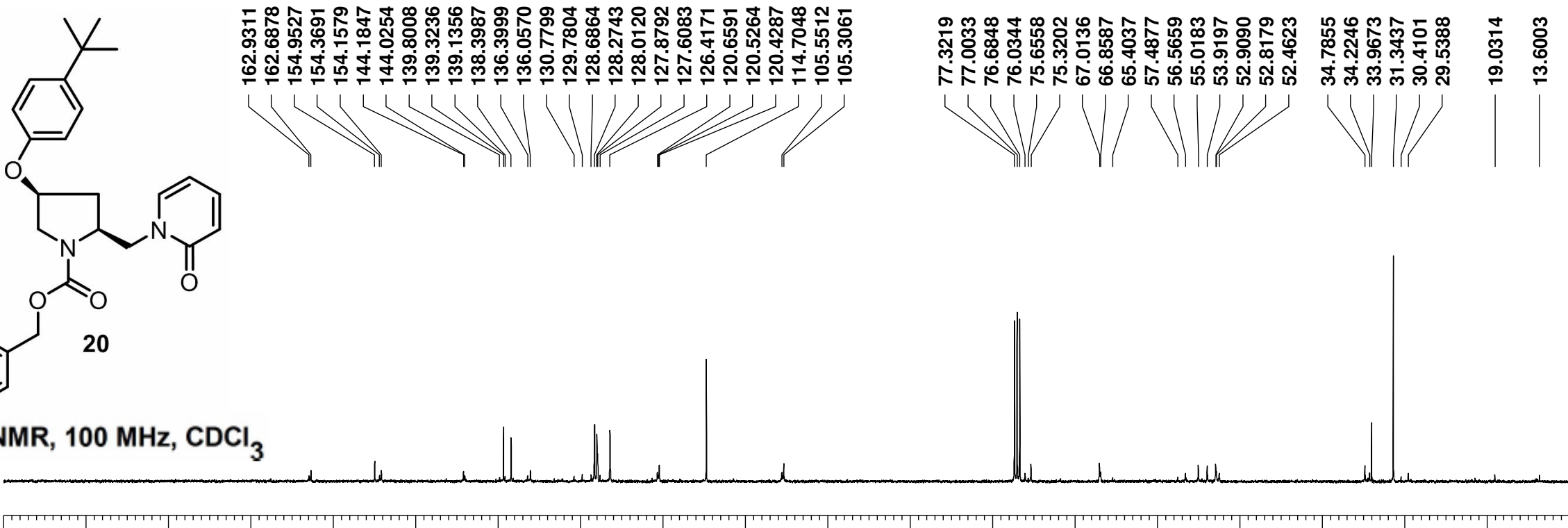

19

$180 \quad 170$

170160

150140

$\begin{array}{lll}130 & 120\end{array}$

20110

100

9080

$\begin{array}{llll}80 & 70 & 60 & 50\end{array}$

$\begin{array}{llll}0 & 40 & 30 & 20\end{array}$ 

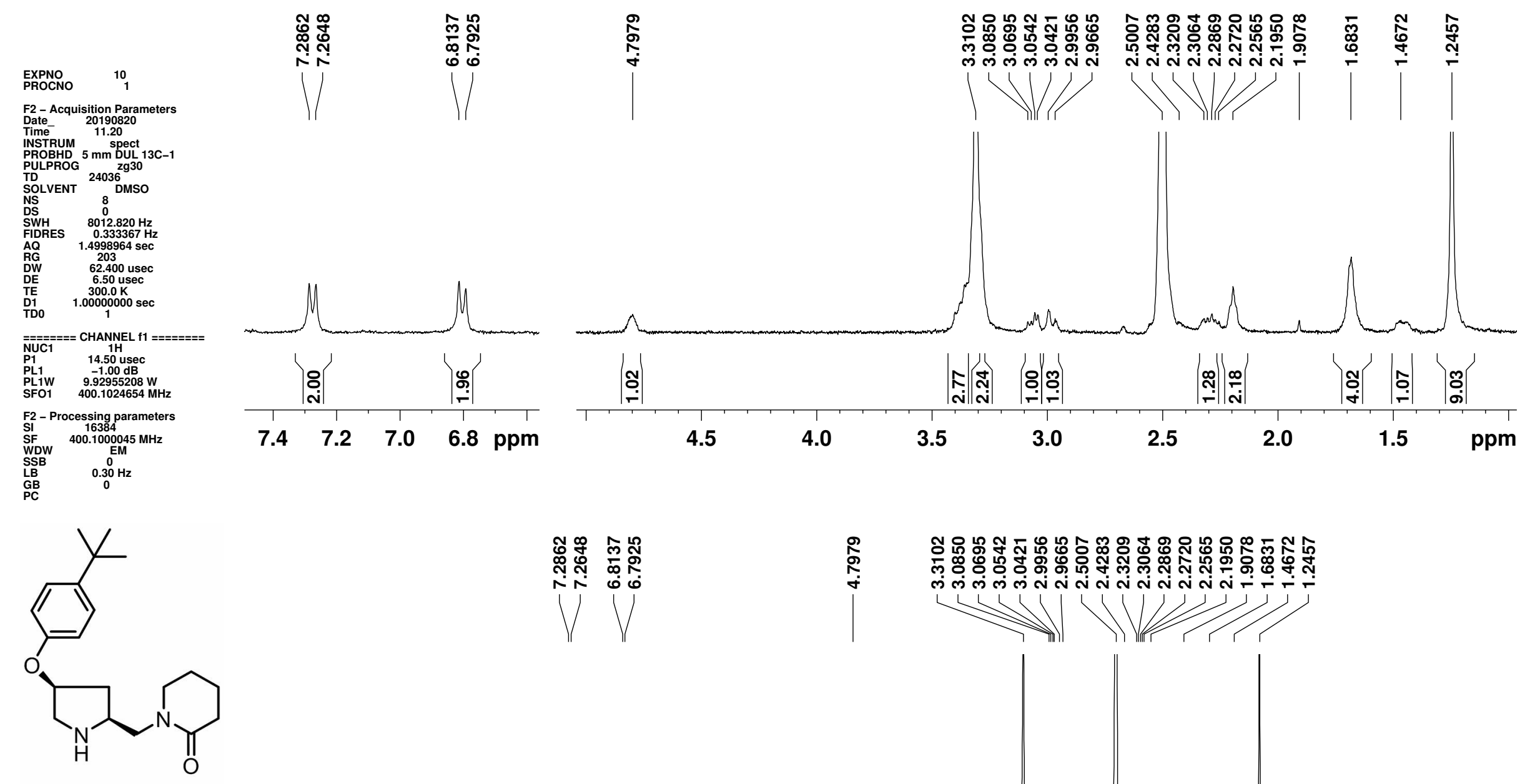

3

${ }^{1} \mathrm{H}$ NMR, $400 \mathrm{MHz}$, DMSO-d 6
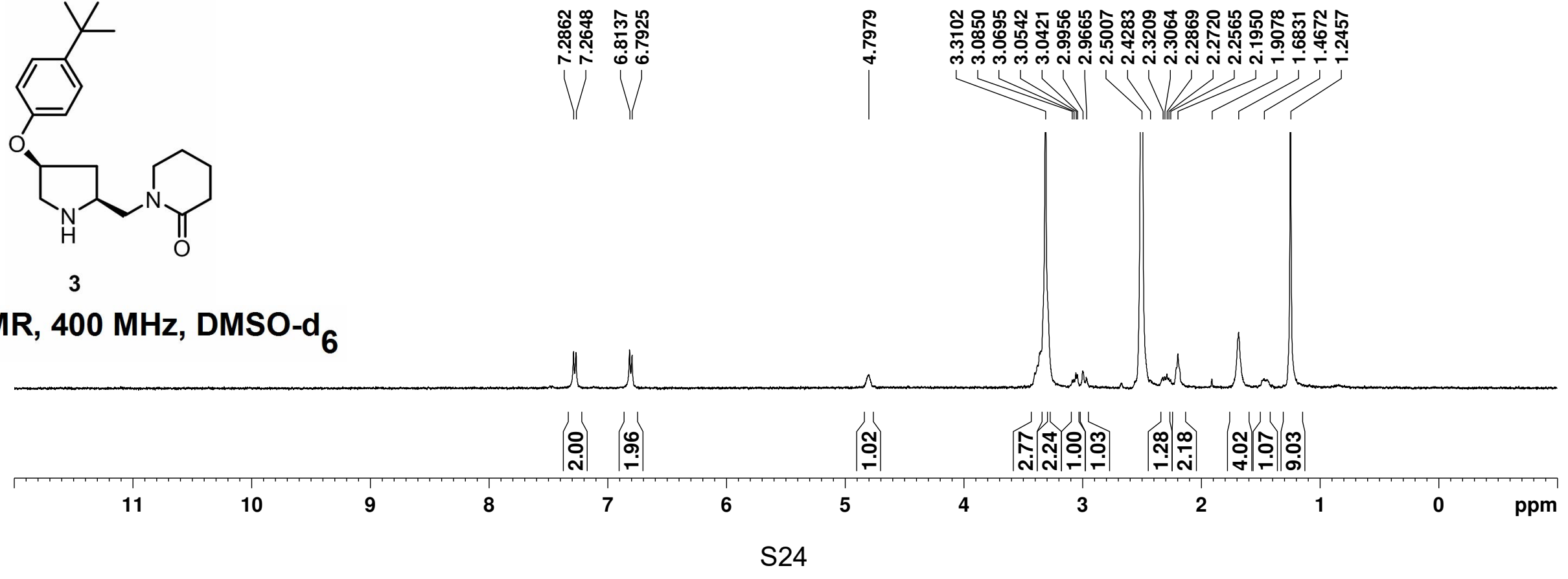

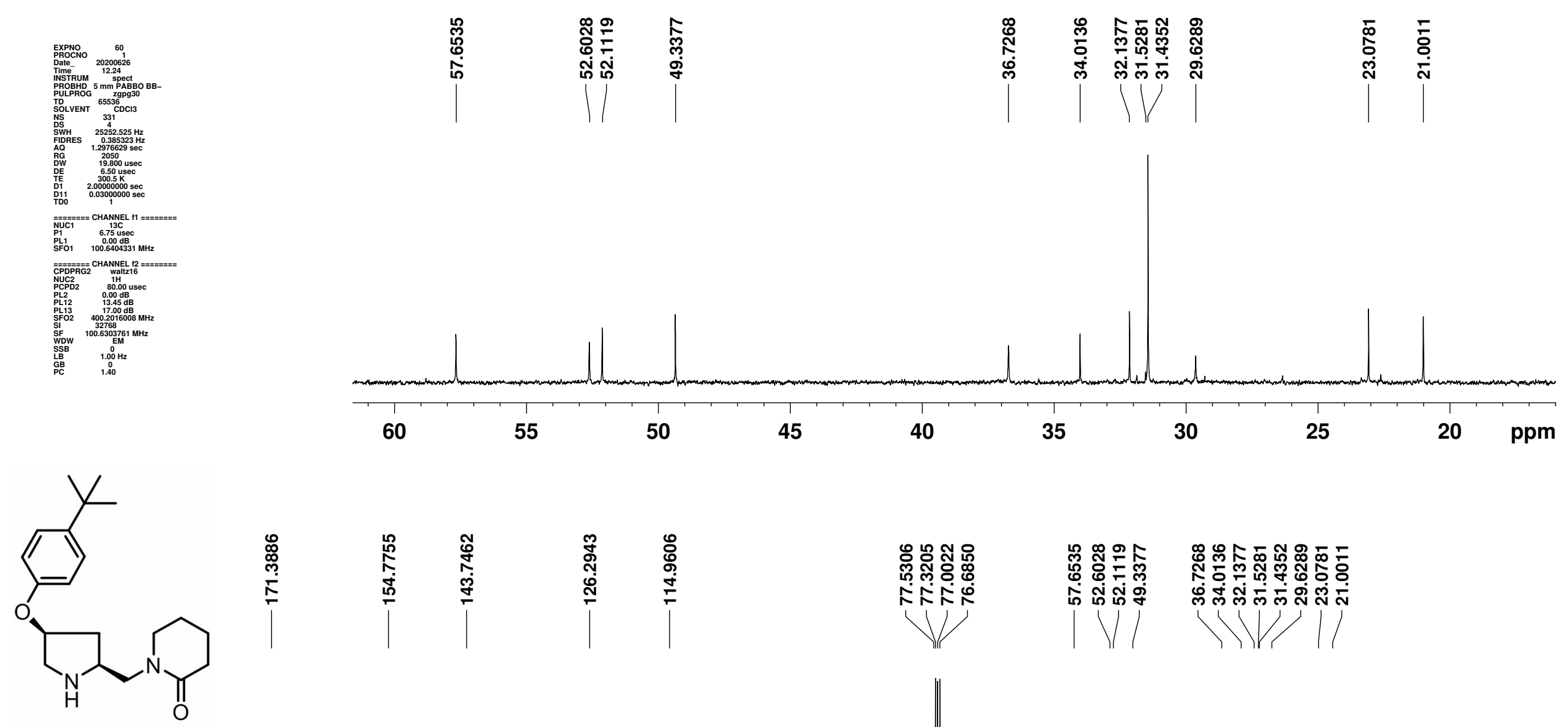

3

${ }^{13} \mathrm{C}\left\{{ }^{1} \mathrm{H}\right\} \mathrm{NMR}, 100 \mathrm{MHz}, \mathrm{CDCl}_{3}$ 

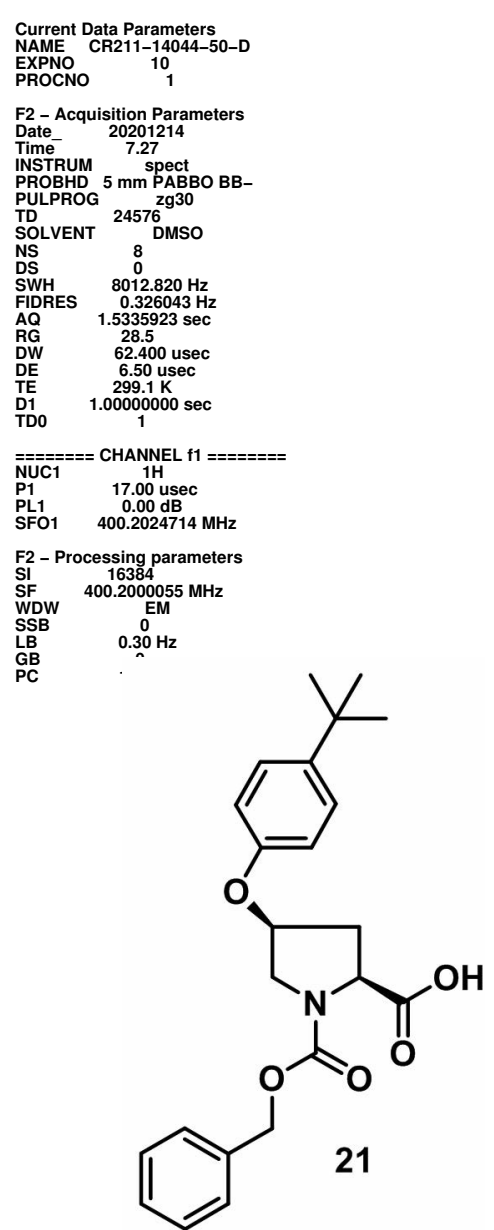

${ }^{1}$ H NMR, 400 MHz, DMSO-d 6

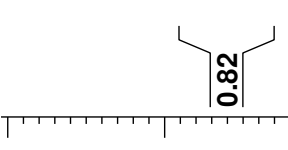

13

10

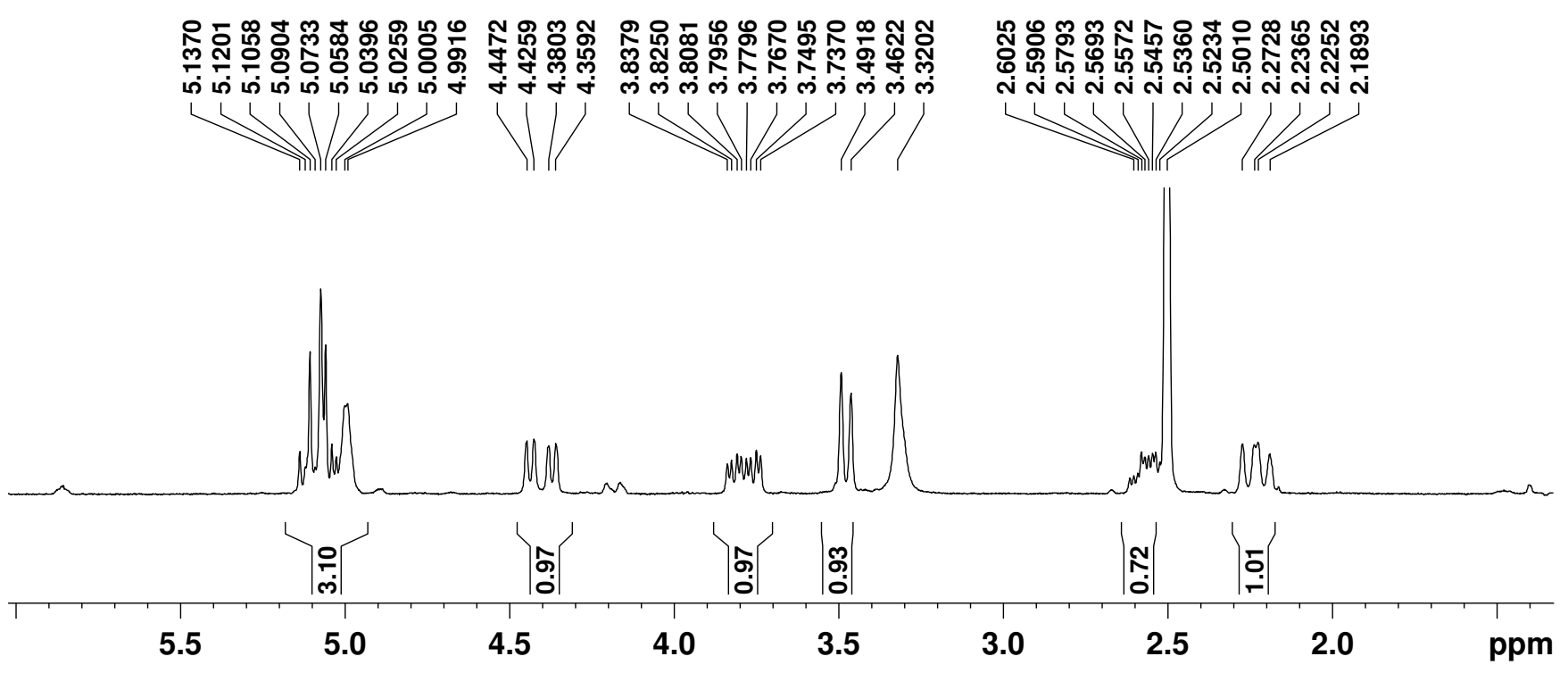

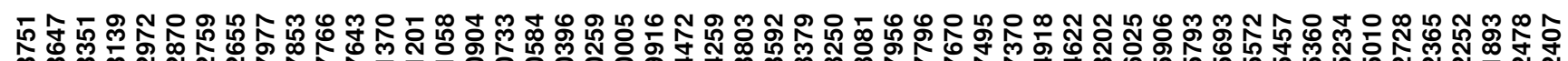

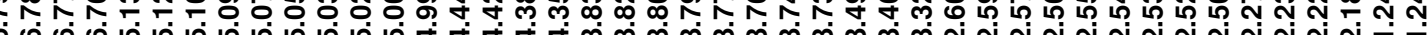
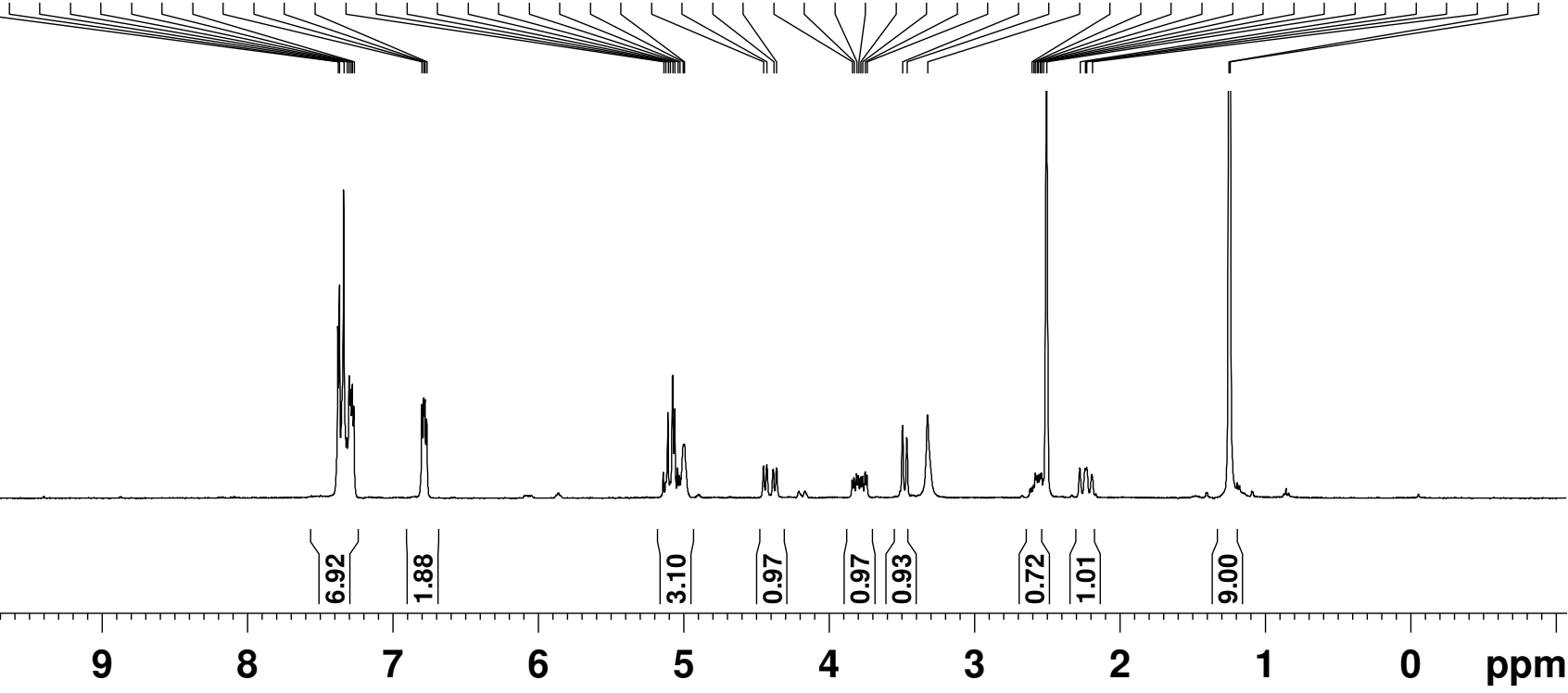


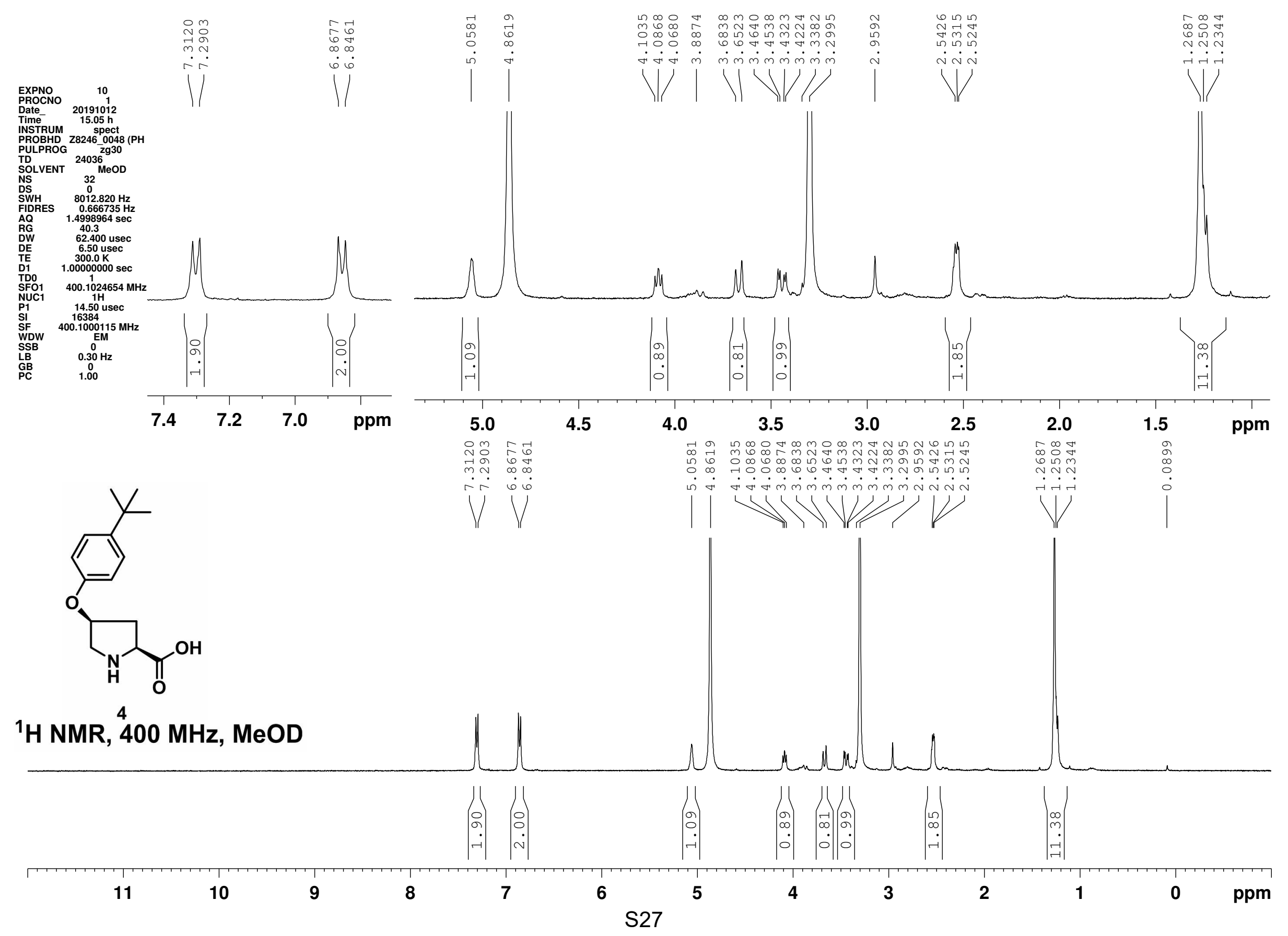




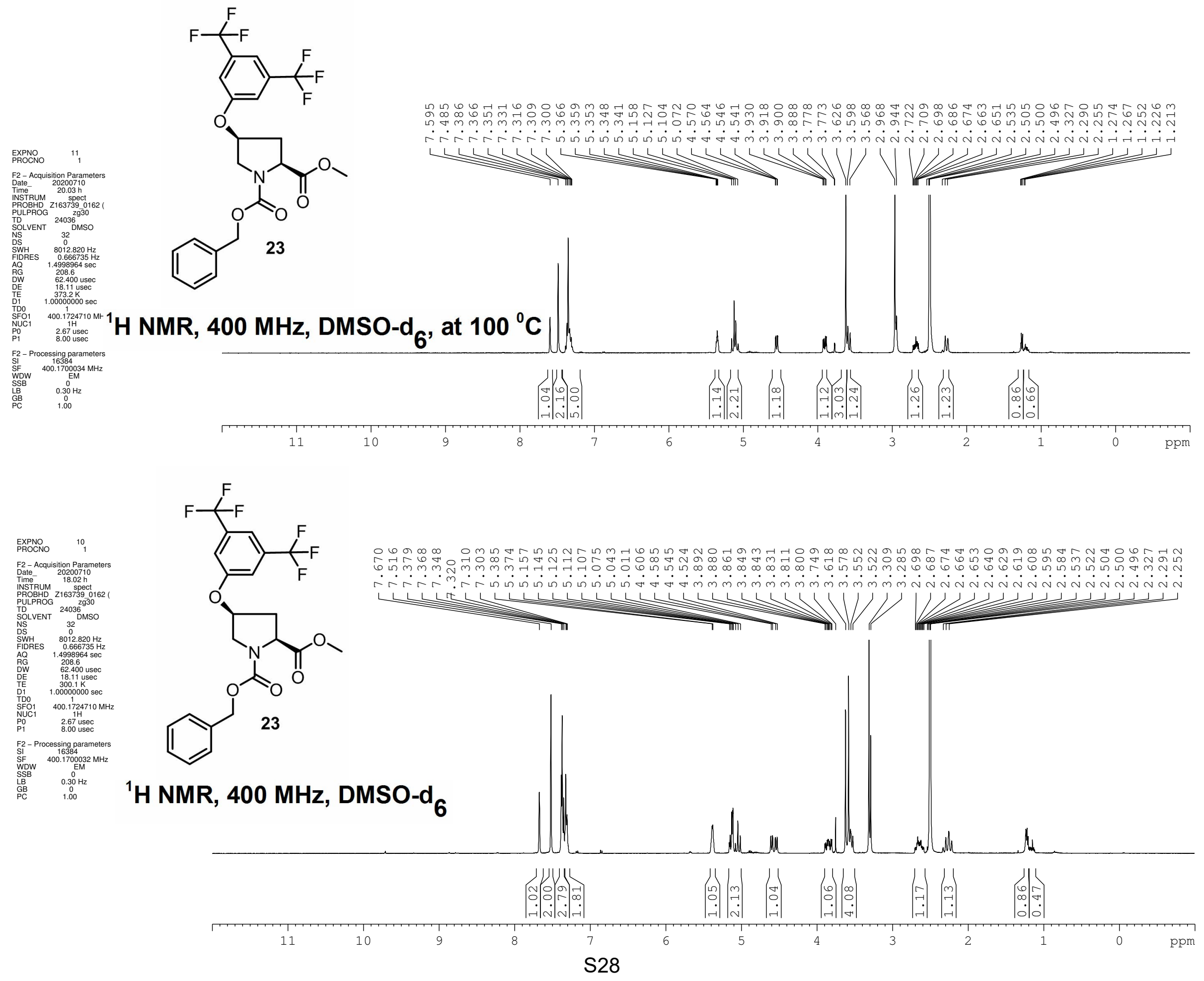




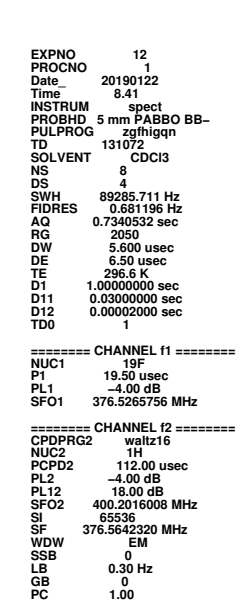

II
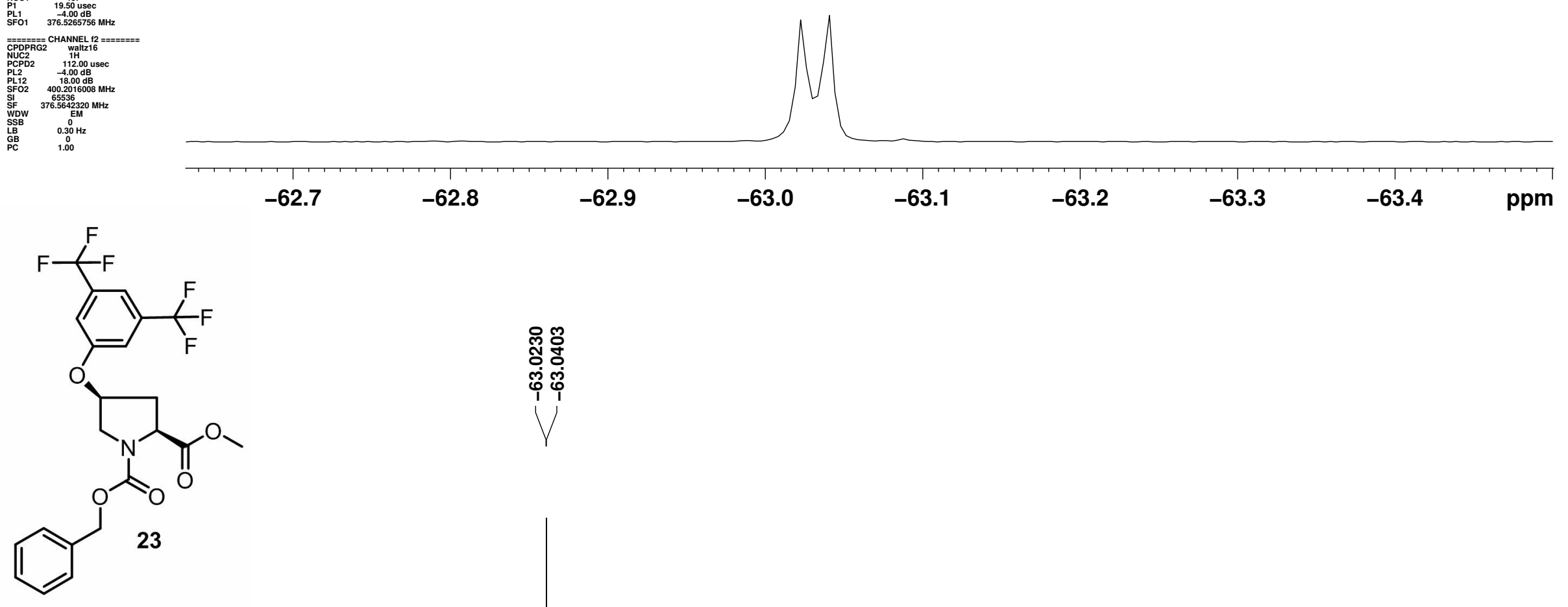

${ }^{19} \mathrm{~F} \mathrm{NMR}, 376 \mathrm{MHz}, \mathrm{CDCl}_{3}$

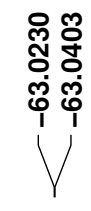

0

$-20$

$-40$

$-60$

$-80$

$-100$

$-120$

$-140$

$-160$

$-180$ 
TCG Lifesciences Private Limited

\section{Kolkata}

NAME CR211-12109-17-P

$\begin{array}{lc} & 60 \\ \text { EXPNO } & 60 C N O \\ \text { Pate } & 20200713 \\ \text { Dime } & 8.10 \mathrm{~h}\end{array}$

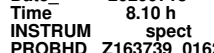

PROBHD Z163739 0162(

TD ${ }_{65}$ zgpog 30

NS 596

$\begin{array}{lr}\text { SWH } & 24038.461 \mathrm{~Hz} \\ \text { FIDRES } & 0.733596 \mathrm{~Hz} \\ \text { AD } & 13.31989 .62\end{array}$

$\begin{array}{lc}\text { AQ } & 1.3631988 \mathrm{sec} \\ \text { RG } & 208.6\end{array}$

$\begin{array}{ll}\text { DW } & 20.800 \text { usec } \\ \text { DE } & 6.50 \text { usec } \\ \text { DE } & 297.3 \mathrm{~K}\end{array}$

$\begin{array}{ll}\text { TE } & 297.3 \mathrm{~K} \\ \text { D1 } & 2.00000000 \\ \text { D11 } & 0.03000000 \mathrm{sec}\end{array}$

$\begin{array}{ll}\text { TDO } & 1 \\ \text { SFO1 } & 100.6328888 \mathrm{MHz}\end{array}$

$\begin{array}{ll}\text { P0 } & 2.67 \text { usec } \\ \text { P1 } & 8.000 \text { usec } \\ \text { SI } & 32768\end{array}$

$\begin{array}{ll}\text { SI } & 32768 \\ \text { SF } & 100.622804 \mathrm{MH}\end{array}$

$\begin{array}{lc}\text { SSB } & 0 \\ \text { LB } & 1.00 \mathrm{~Hz} \\ \text { GB } & 0\end{array}$

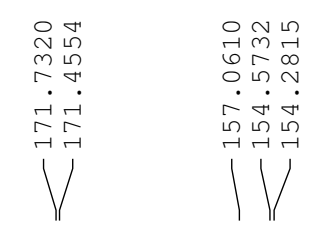

CR211-12109-17-P IN CDCI3-13C
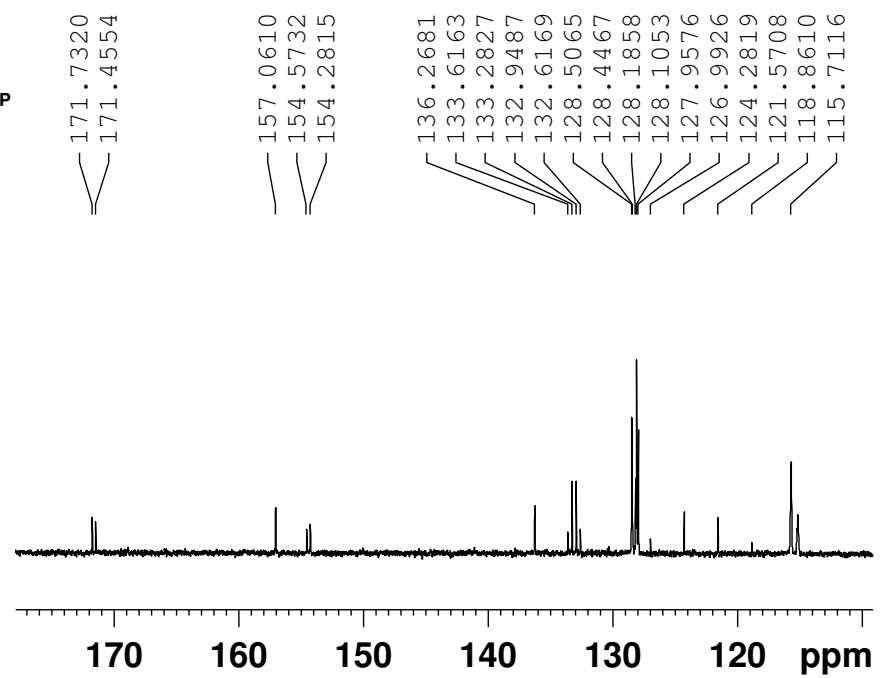
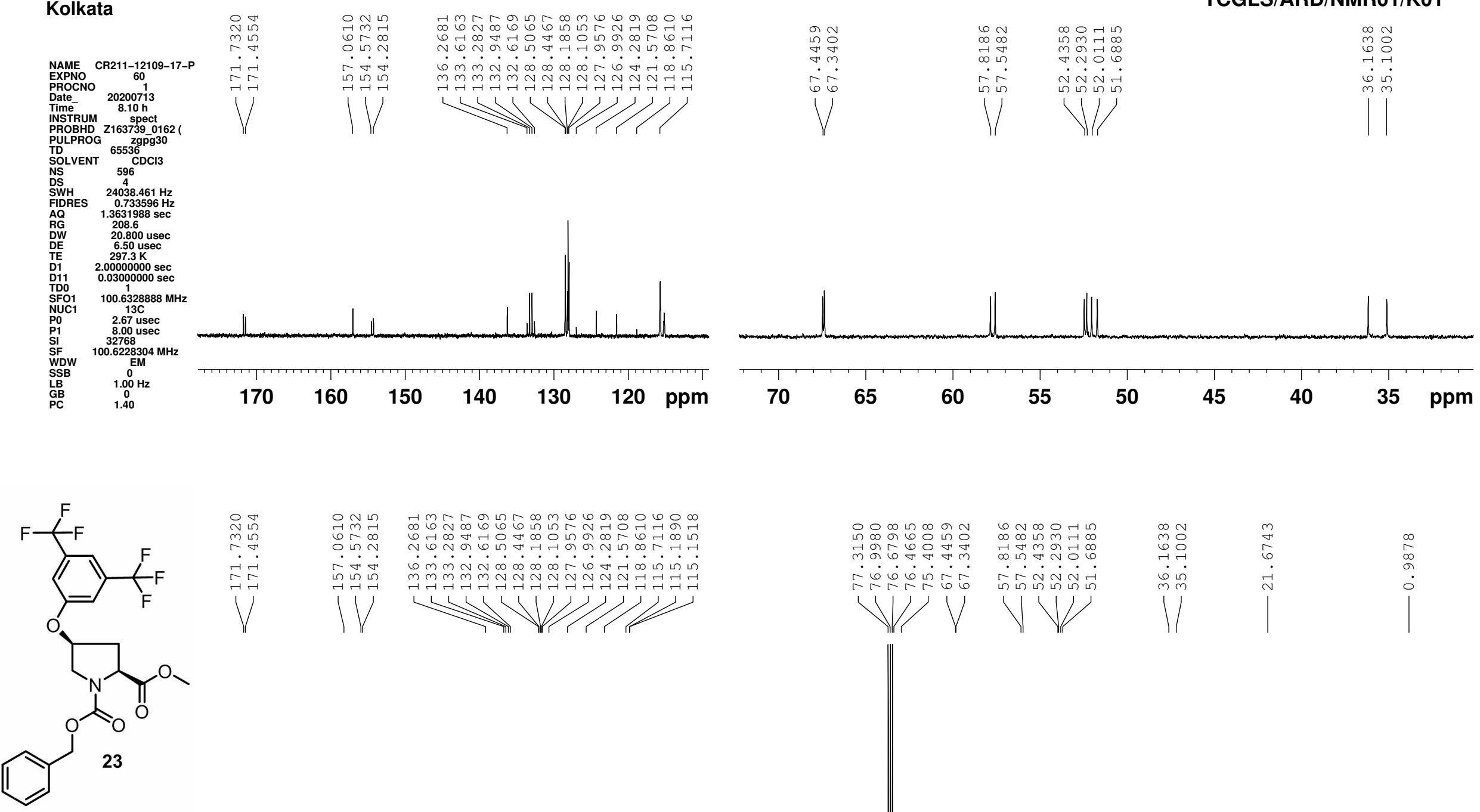

$\left.{ }^{13} \mathrm{C}^{1} \mathrm{H}\right\} \mathrm{NMR}, 100 \mathrm{MHz}, \mathrm{CDCl}_{3}$

190

180

170

150

130

120

110

10090 

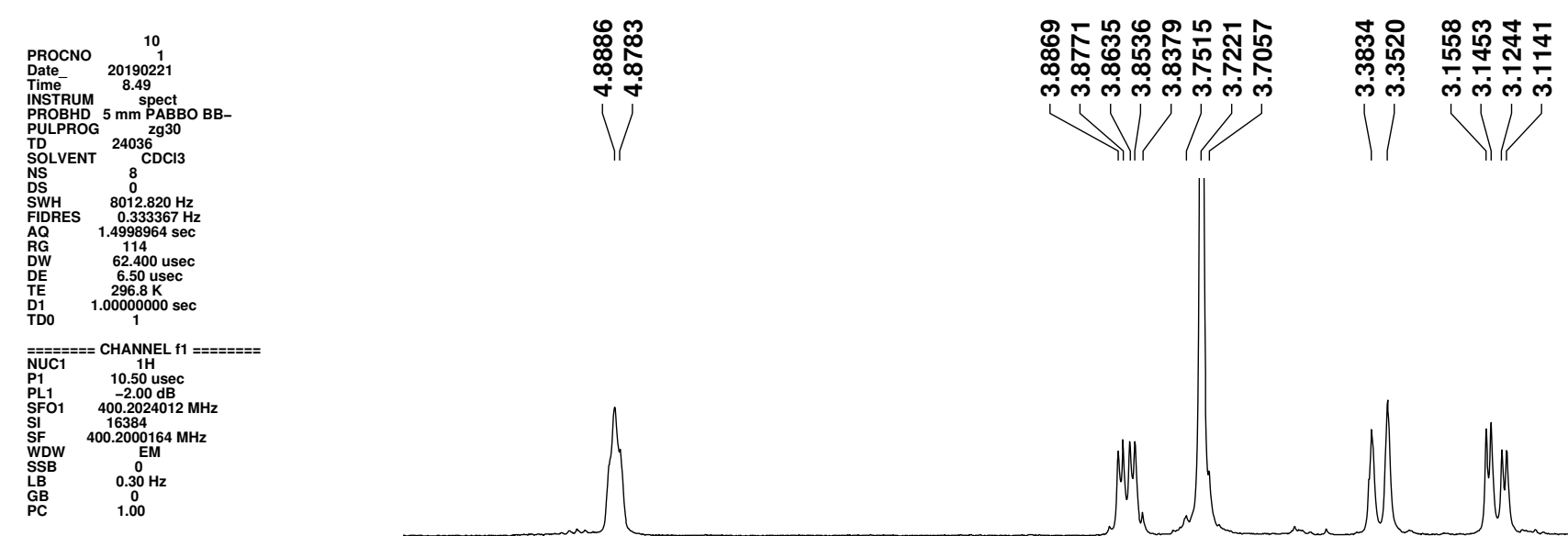

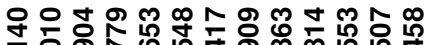

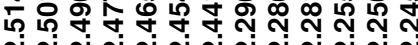
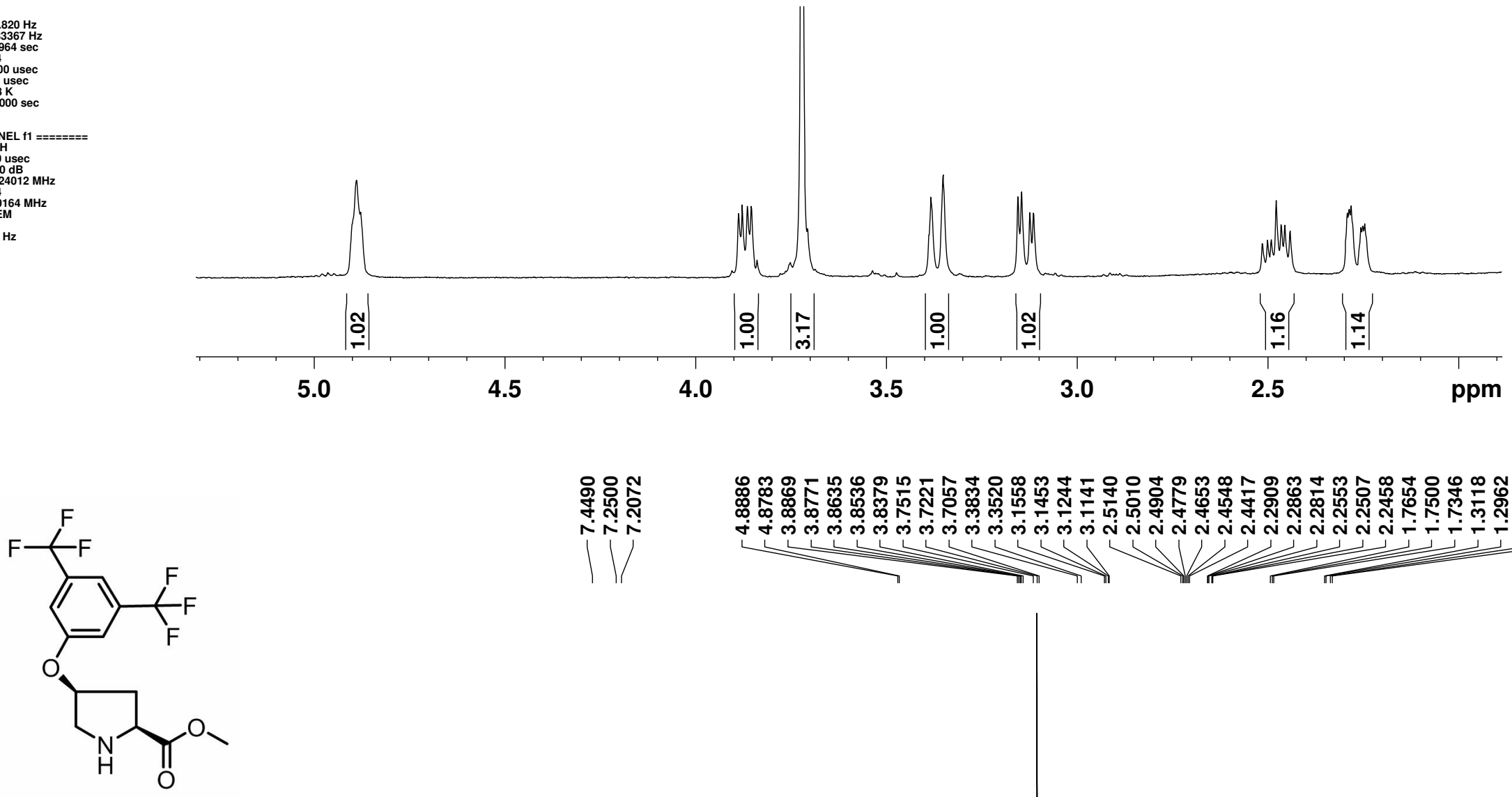

夺莡 商

\&

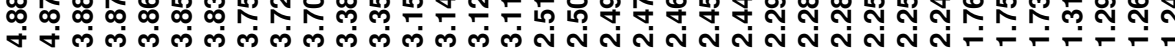

24

${ }^{1} \mathrm{H} \mathrm{NMR}, 400 \mathrm{MHz}, \mathrm{CDCl}_{3}$

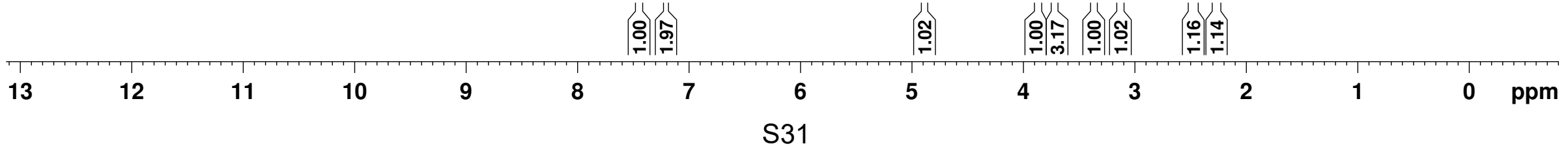



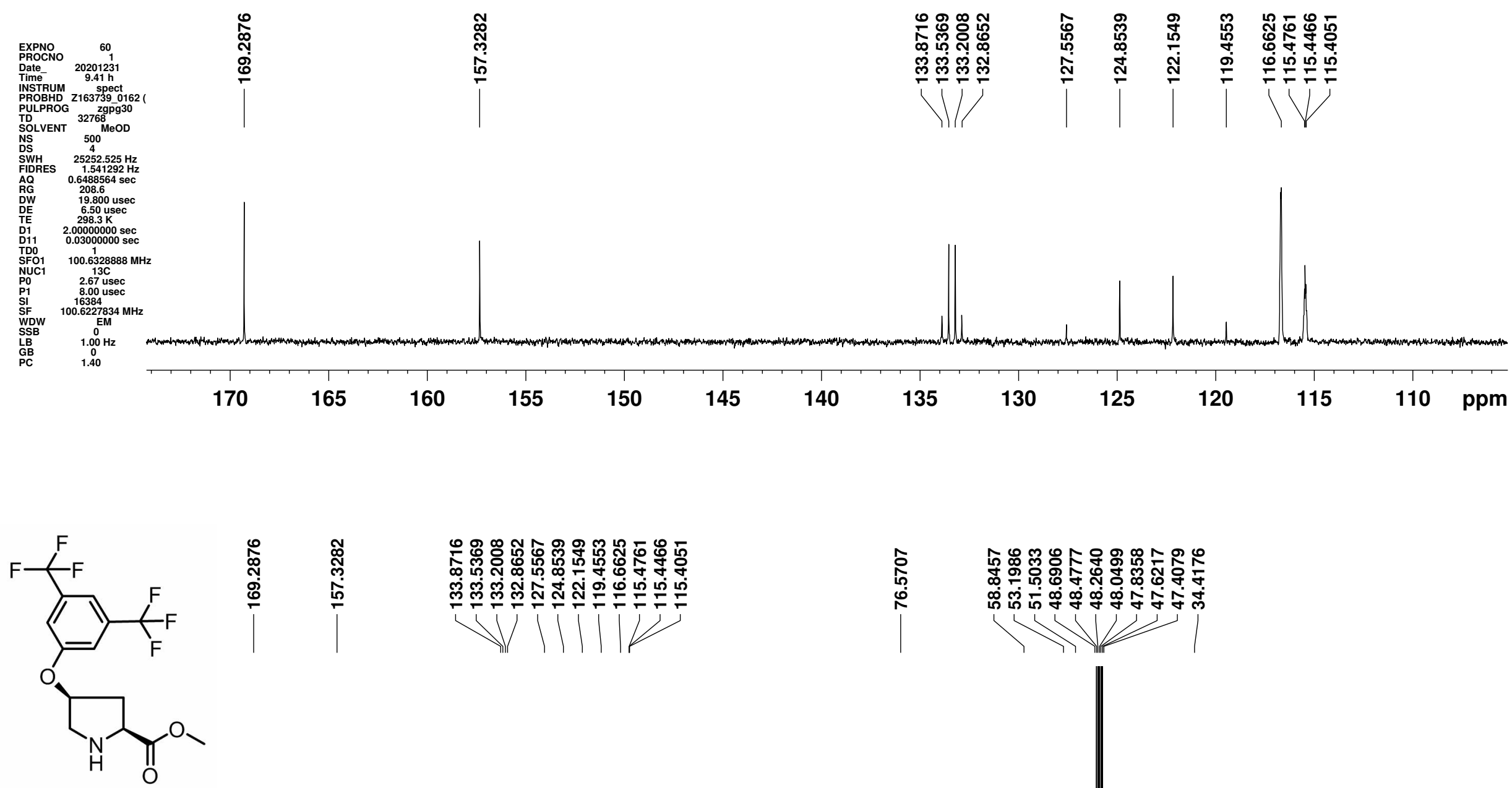

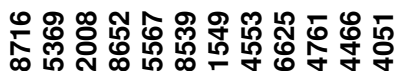

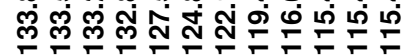

24

${ }^{13} \mathrm{C}\left\{{ }^{1} \mathrm{H}\right\}$ NMR, $100 \mathrm{MHz}, \mathrm{MeOD}$

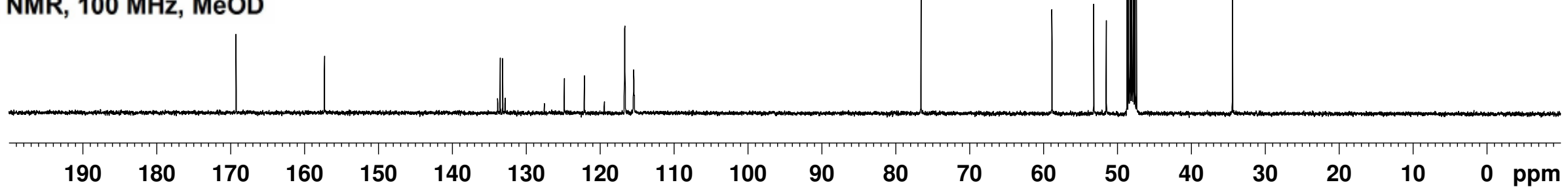



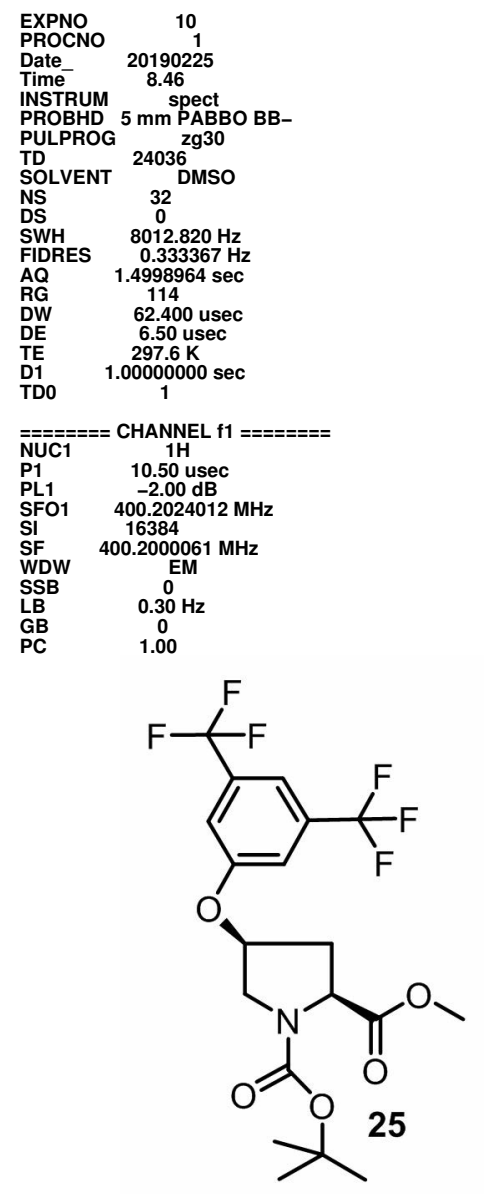
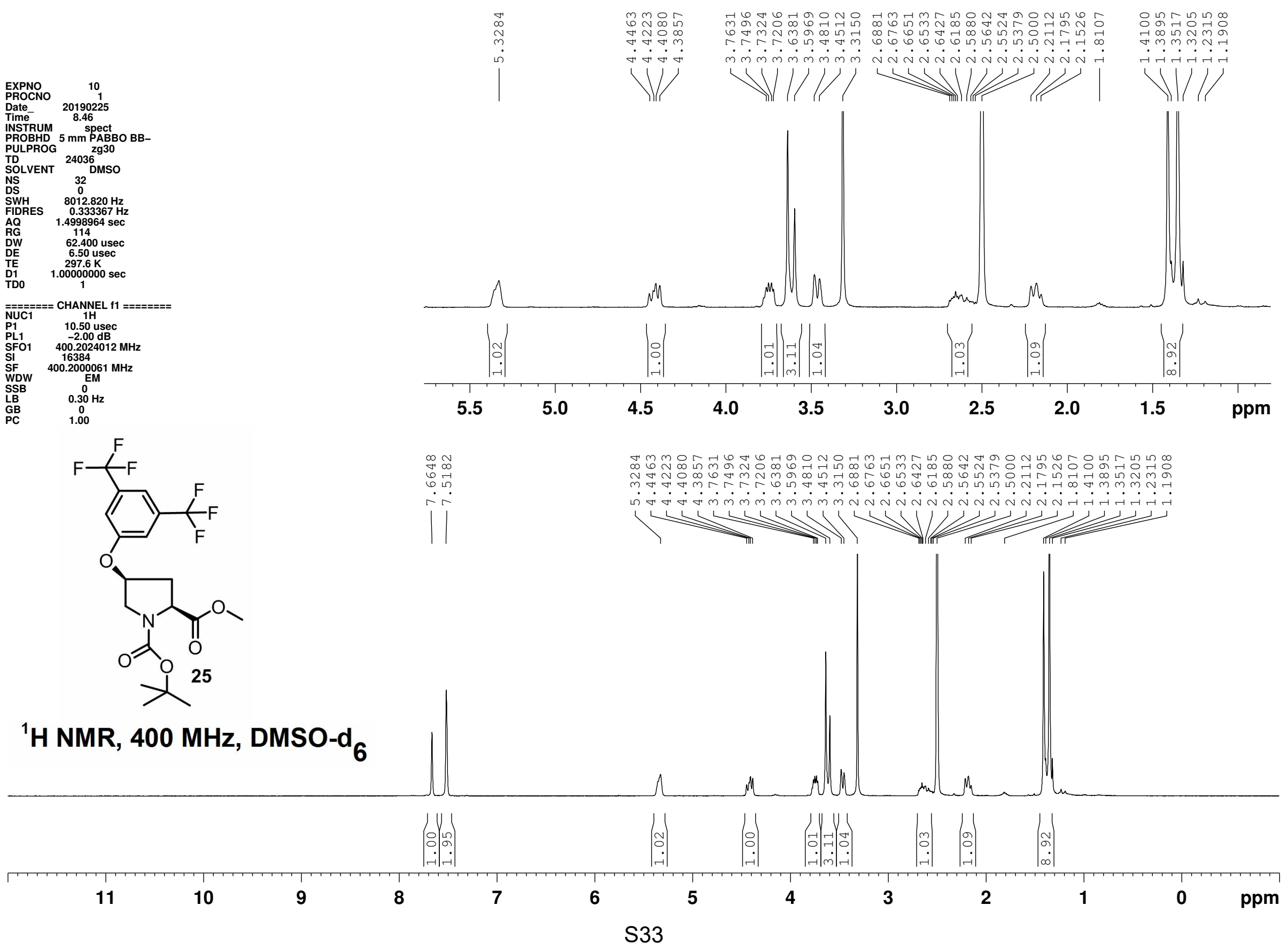


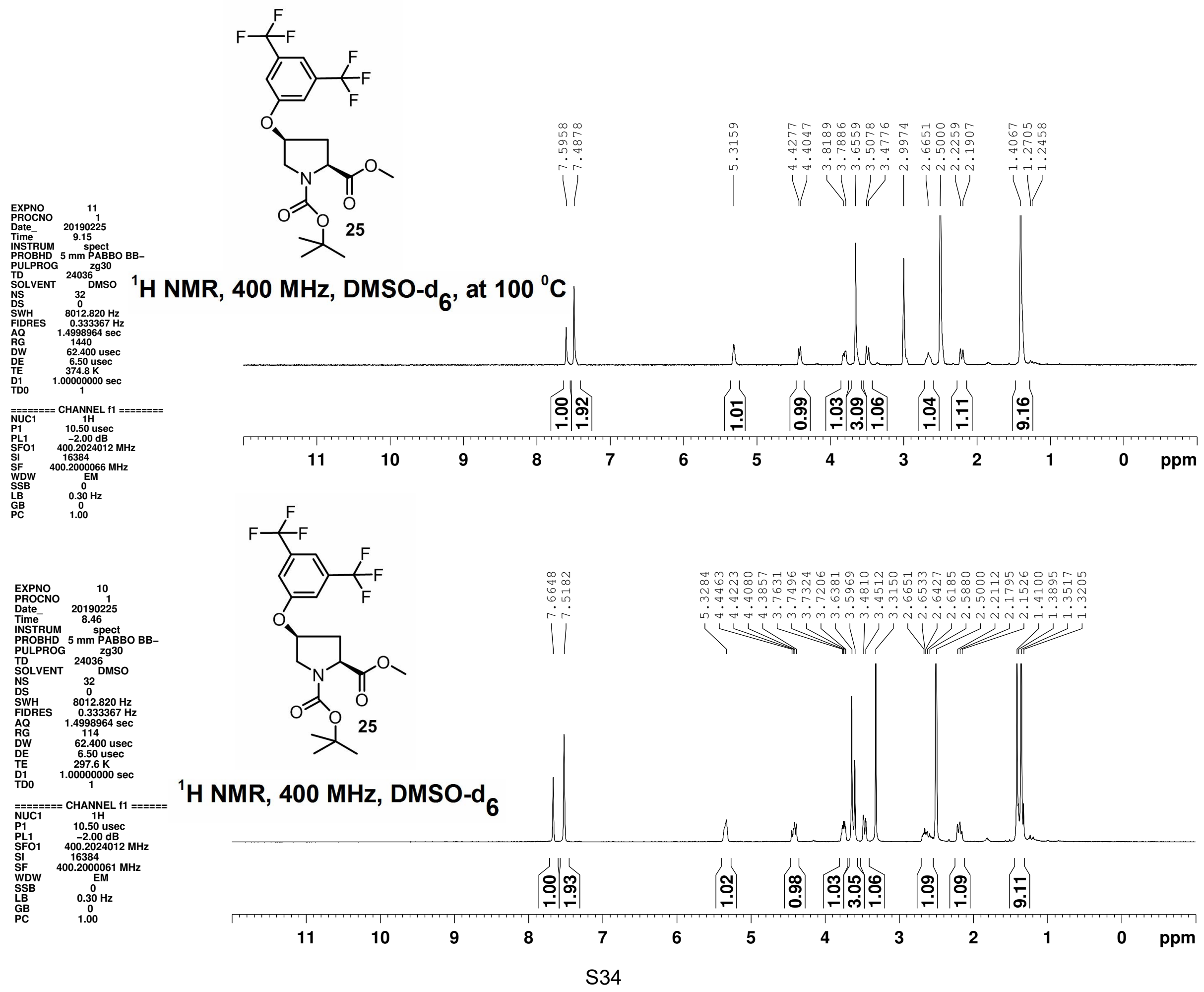




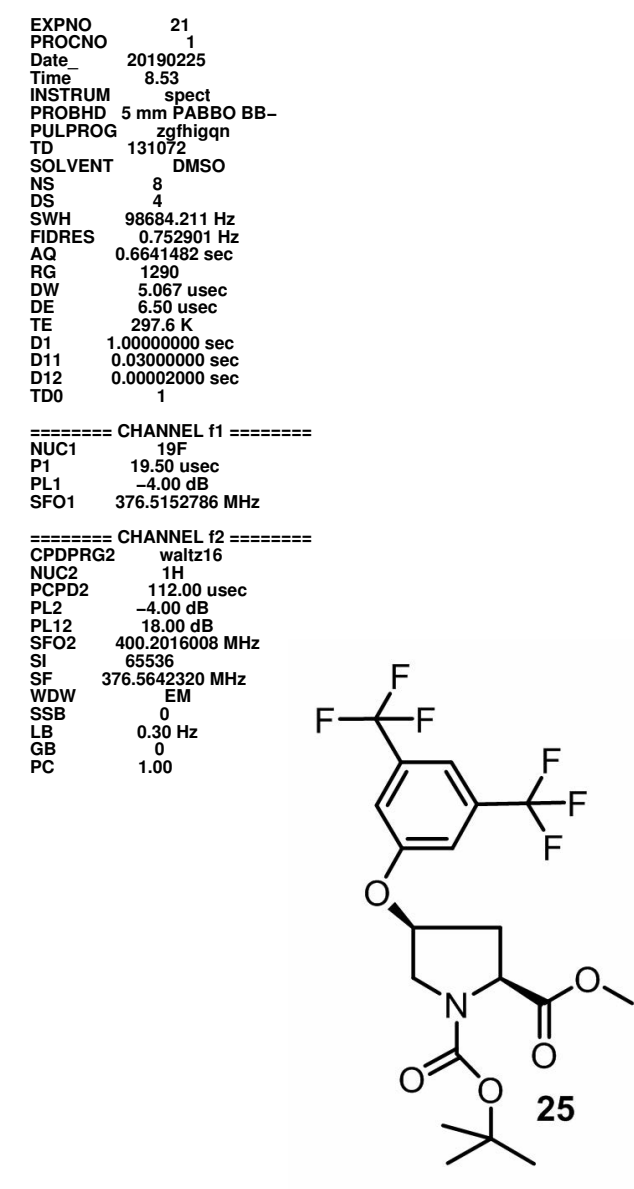

${ }^{19} \mathrm{~F} \mathrm{NMR}, 376 \mathrm{MHz}, \mathrm{CDCl}_{3}$

\begin{tabular}{rlllllllllllll}
\hline-20 & -40 & -60 & -80 & -100 & -120 & -140 & -160 & -180 & -200 & -220 & -240 & ppm
\end{tabular}



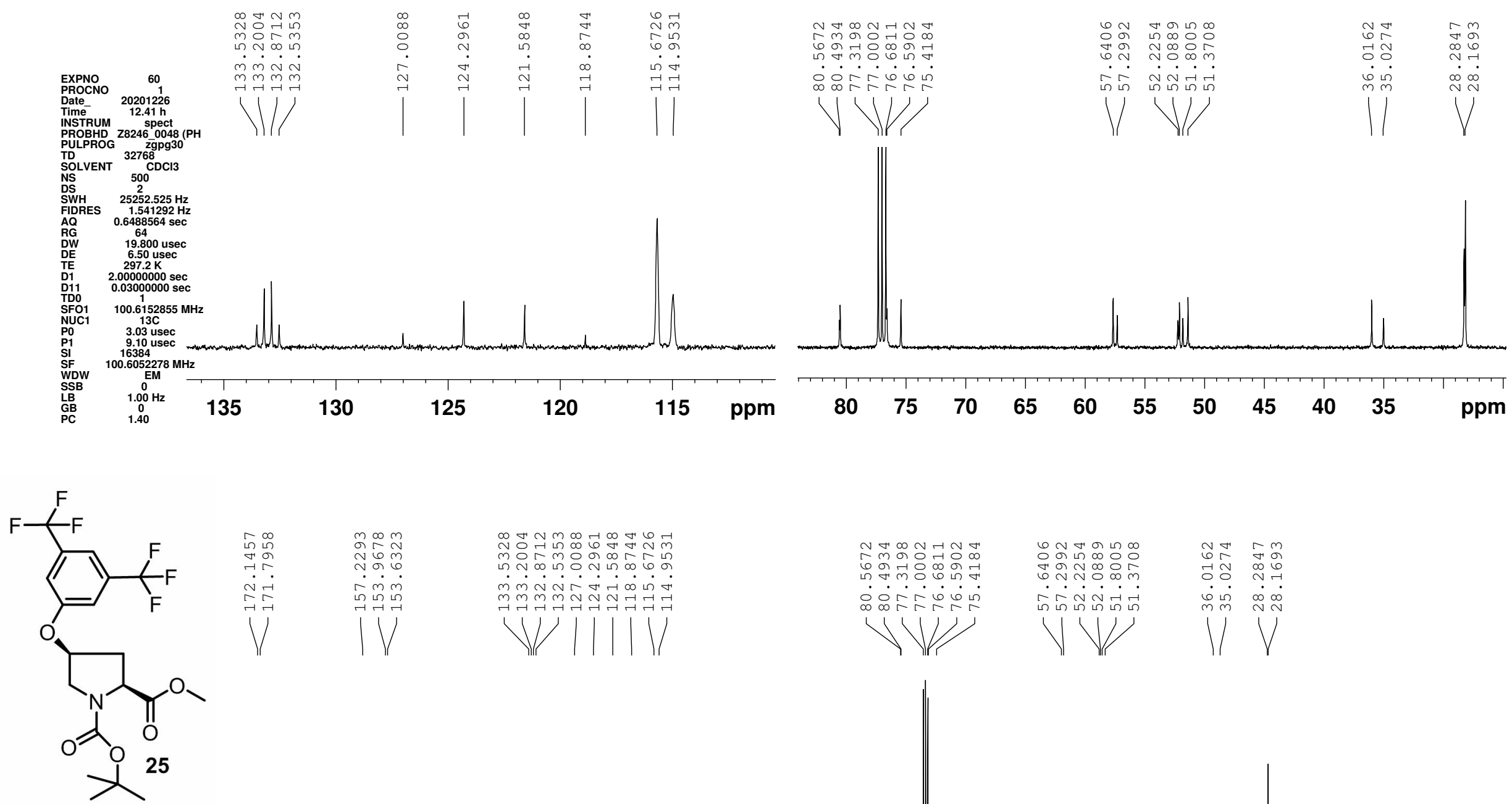

${ }^{13} \mathrm{C}\left\{{ }^{1} \mathrm{H}\right\} \mathrm{NMR}, 100 \mathrm{MHz}, \mathrm{CDCl}_{3}$

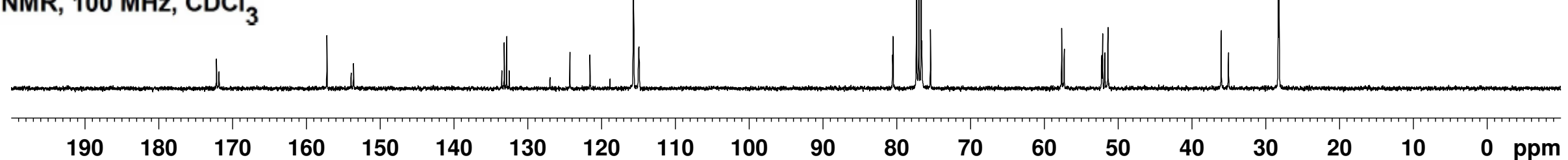



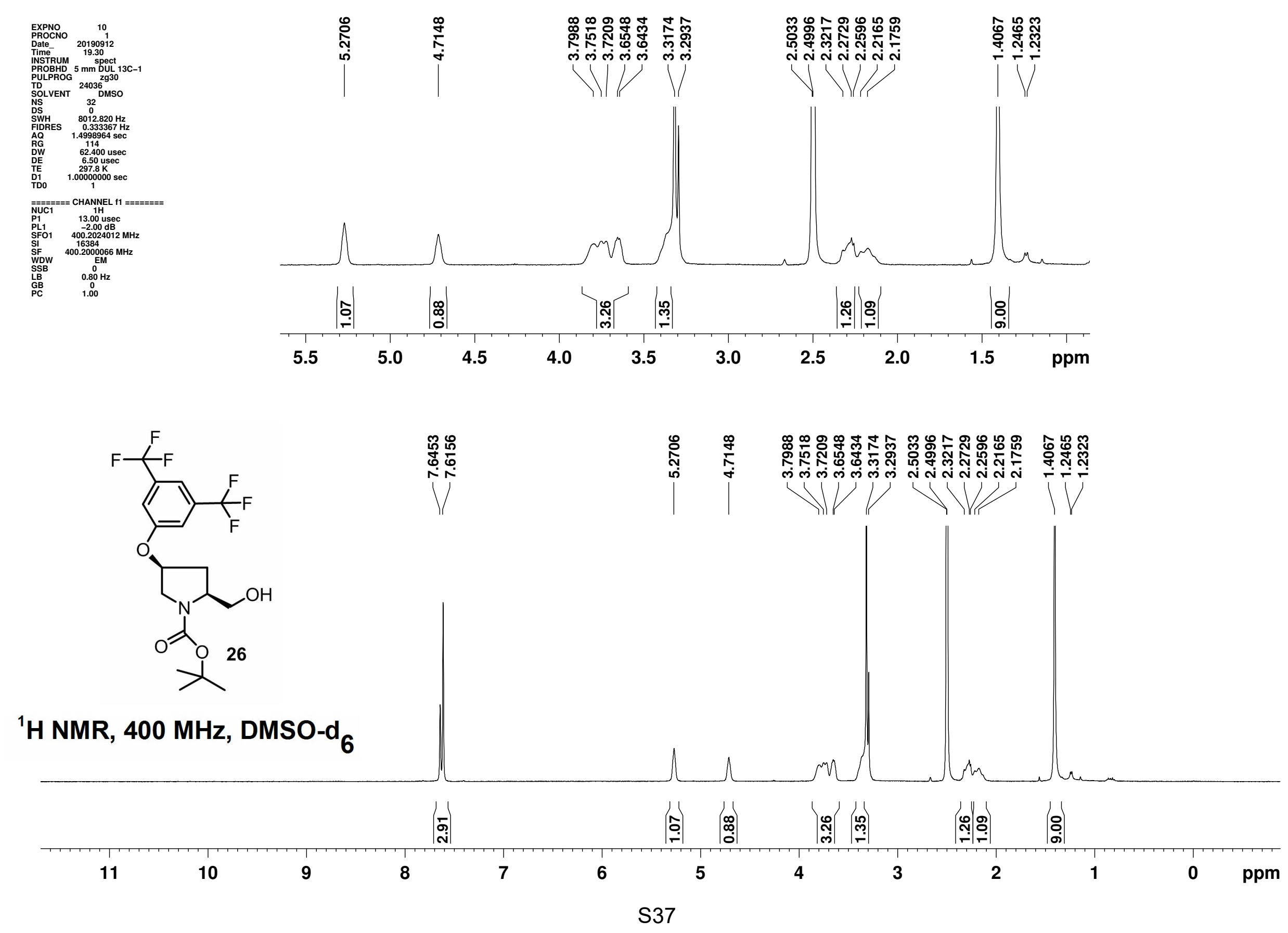


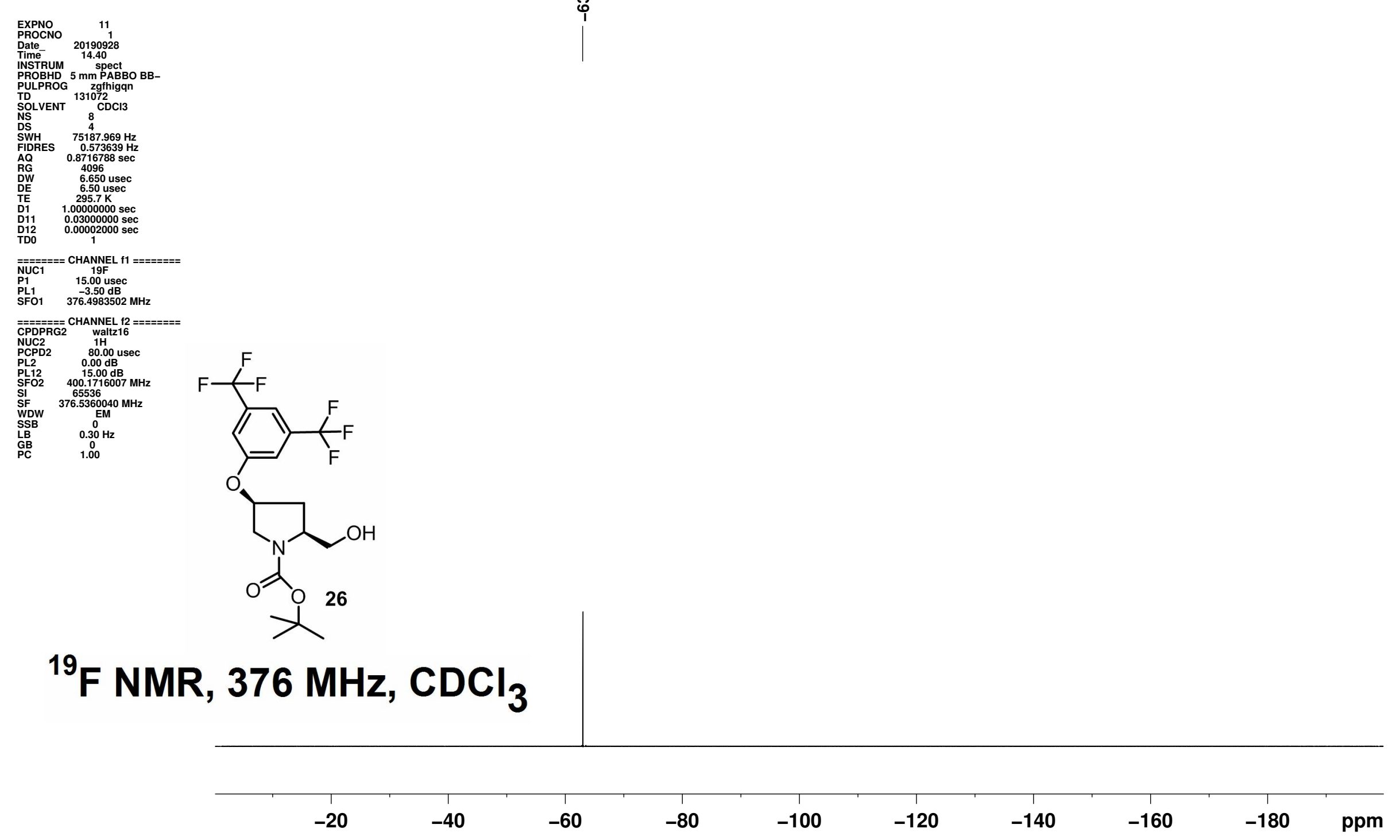



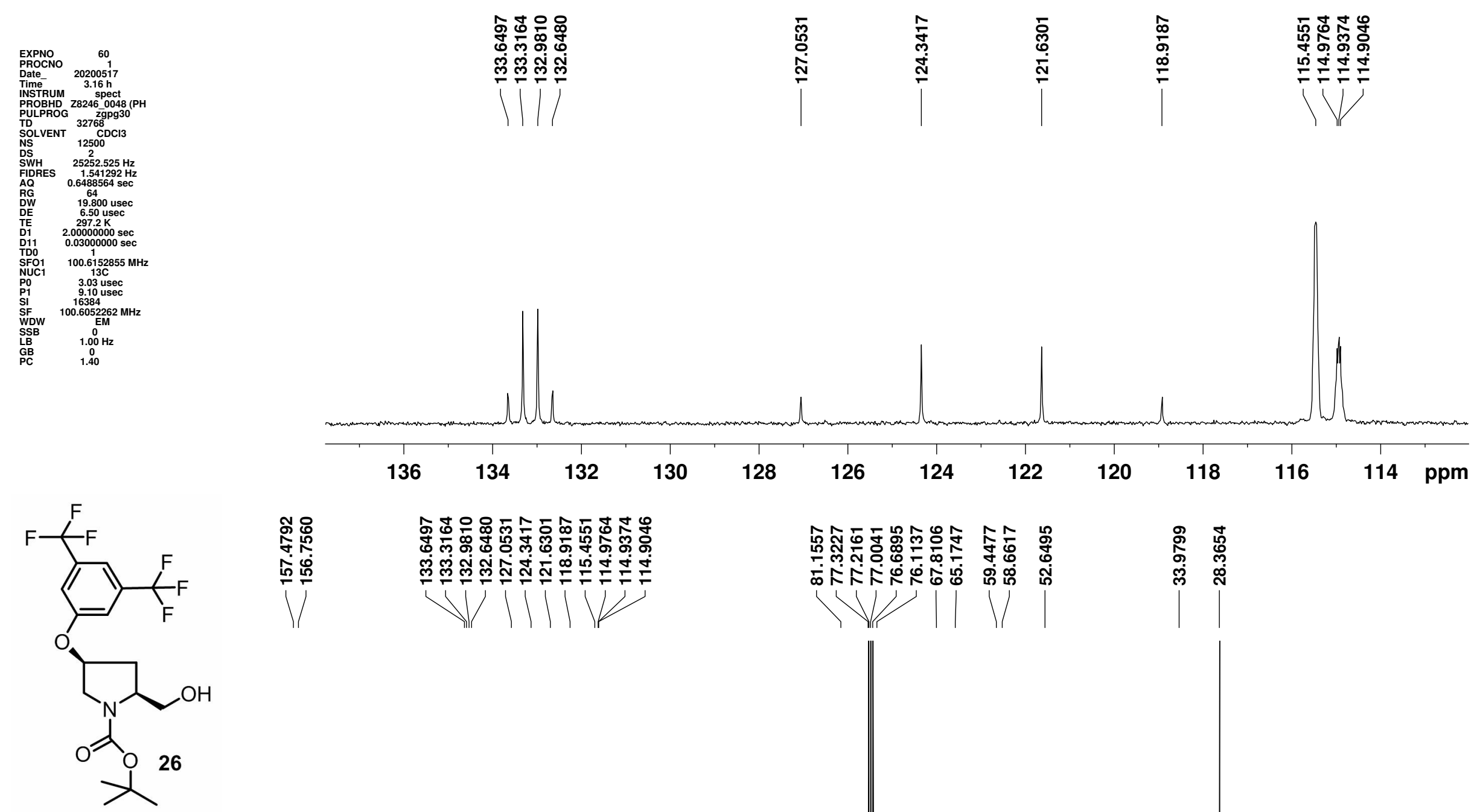

${ }^{13} \mathrm{C}\left\{{ }^{1} \mathrm{H}\right\} \mathrm{NMR}, 100 \mathrm{MHz}, \mathrm{CDCl}_{3}$
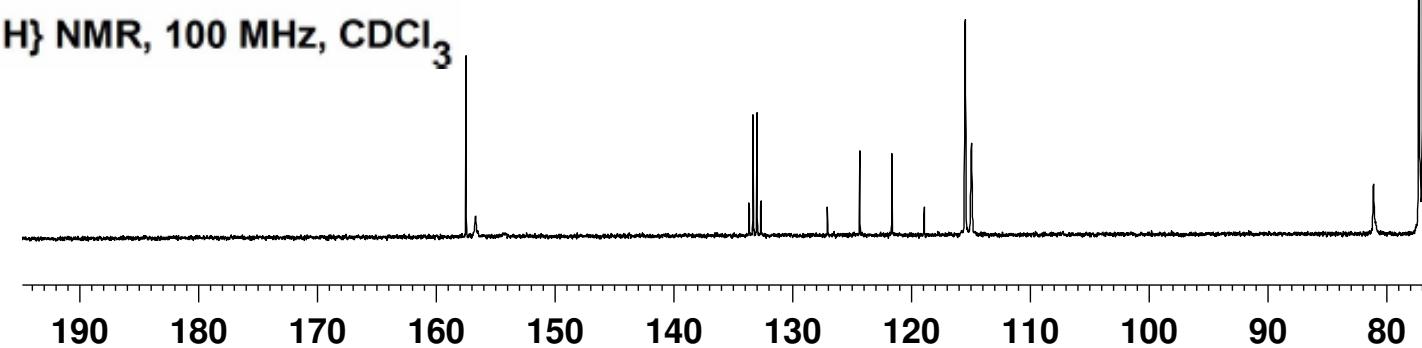

160

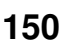

140

130

120

110100

90

80

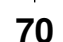

60

50

40

30 


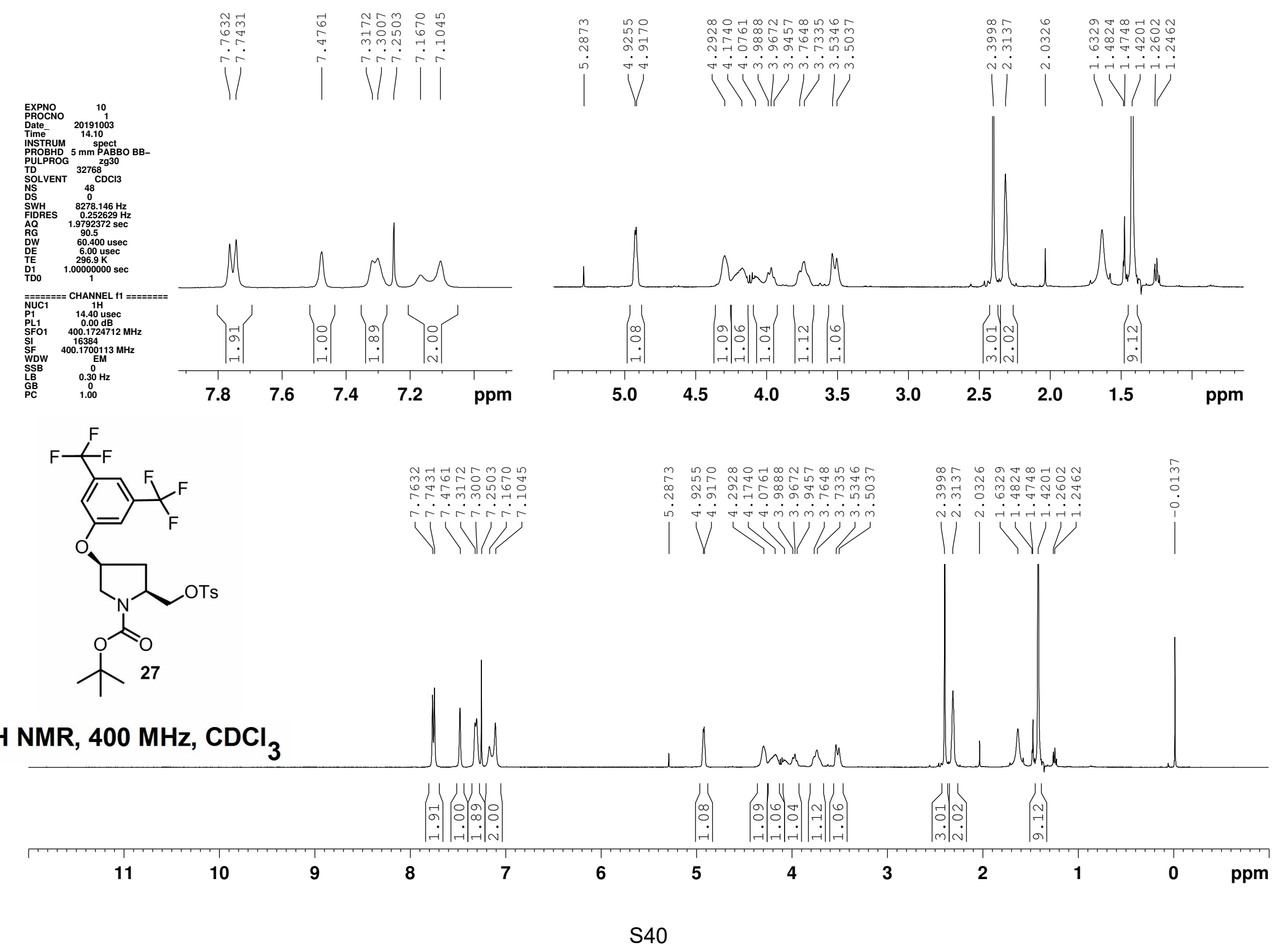




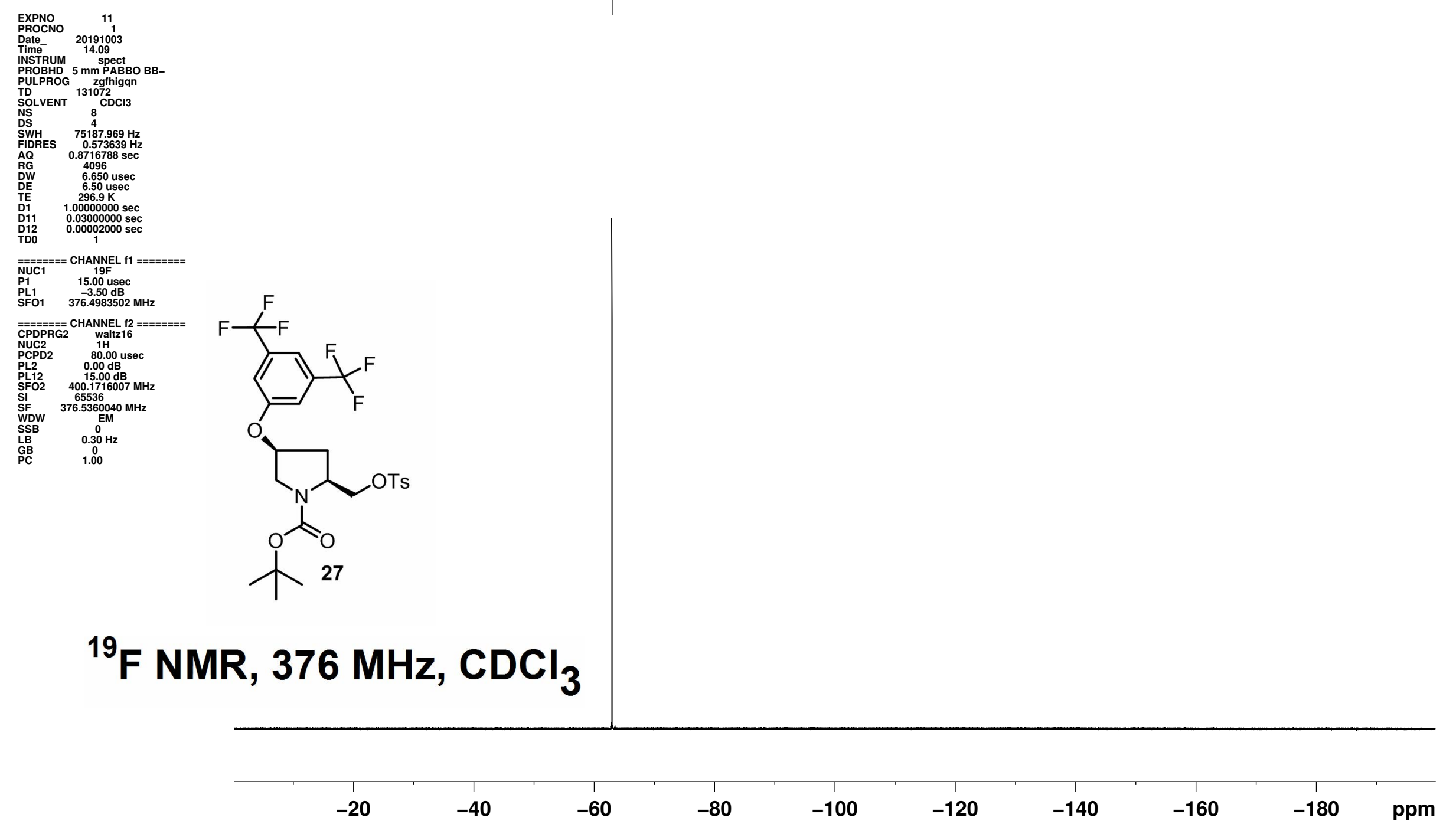



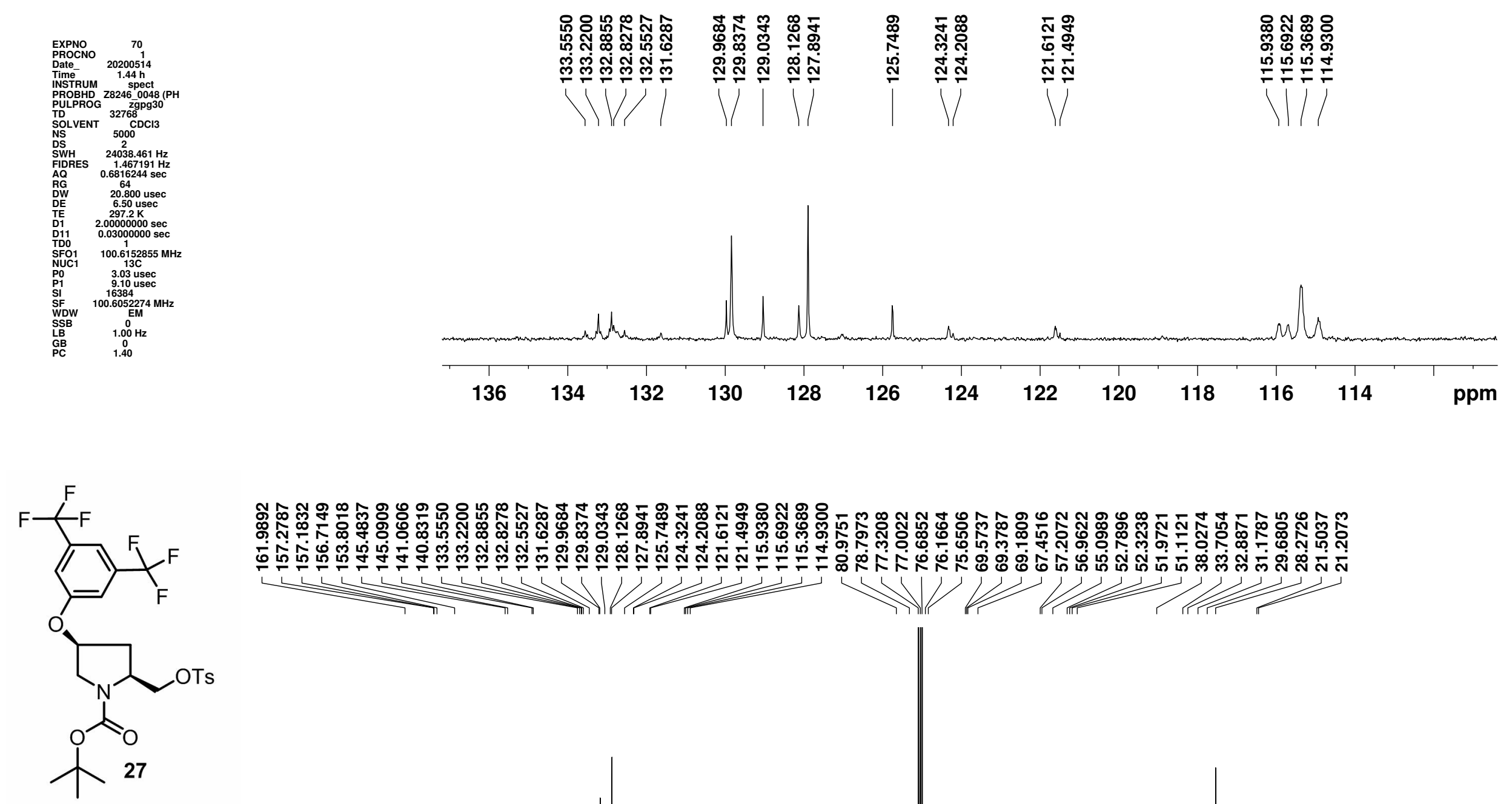

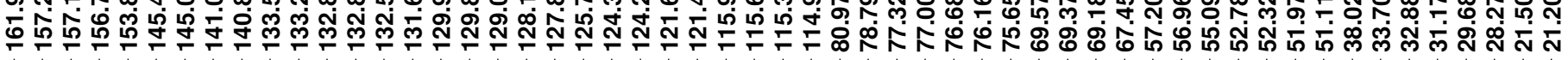

${ }^{13} \mathrm{C}\left\{{ }^{1} \mathrm{H}\right\} \mathrm{NMR}, 100 \mathrm{MHz}, \mathrm{CDCl}_{3}$
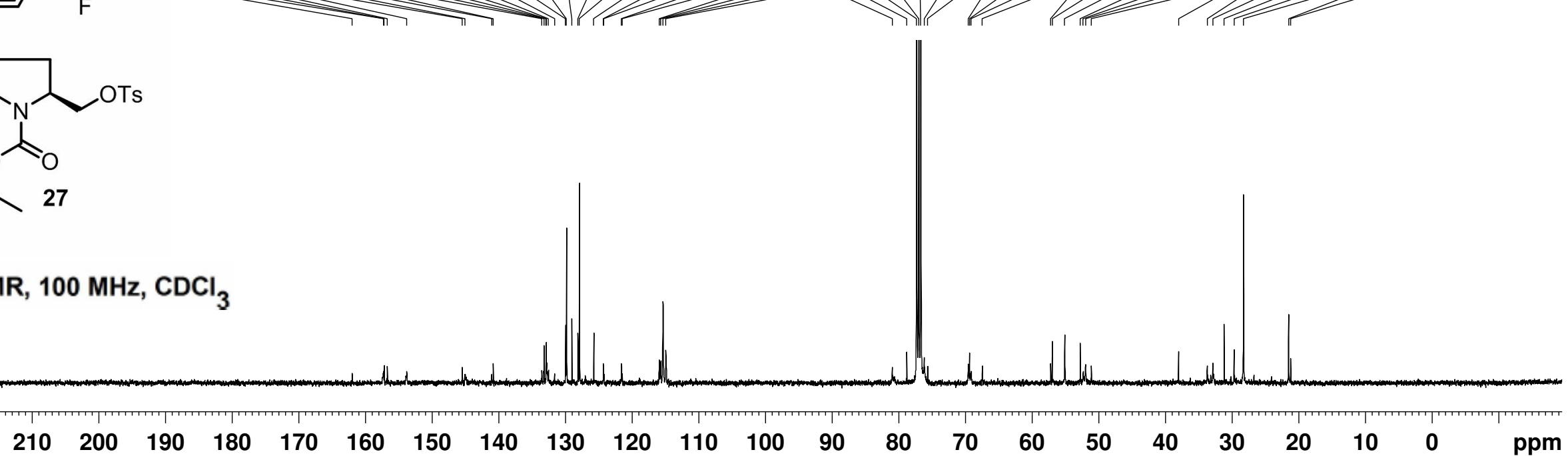

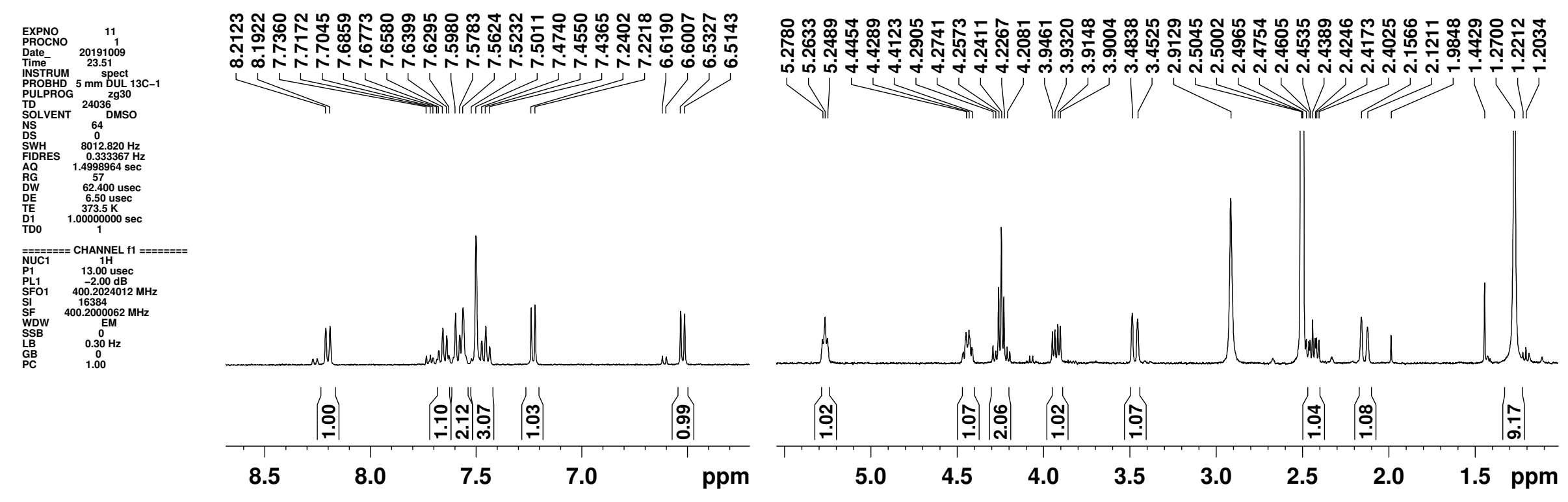

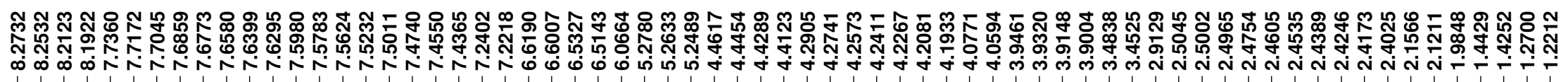

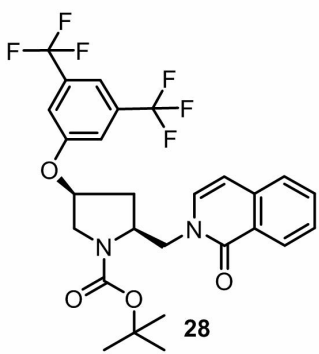

${ }^{1} \mathrm{H}$ NMR, $400 \mathrm{MHz}, \mathrm{DMSO}-\mathrm{d}_{6}$, at $100{ }^{\circ} \mathrm{C}$

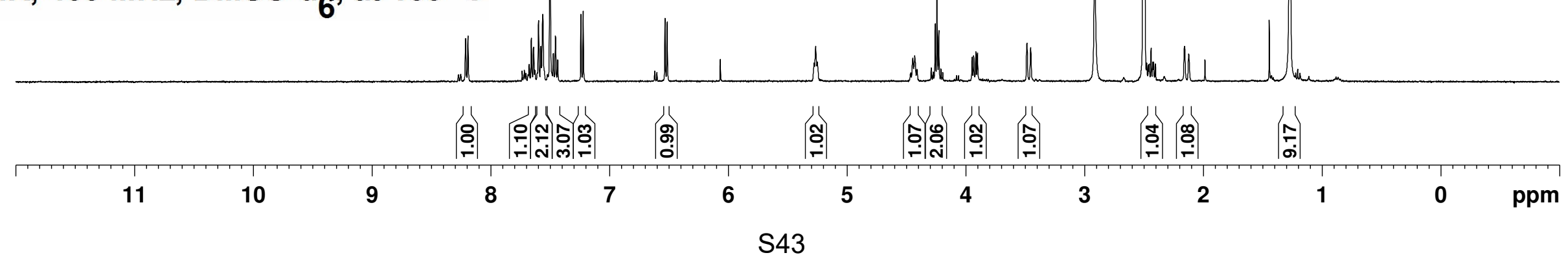



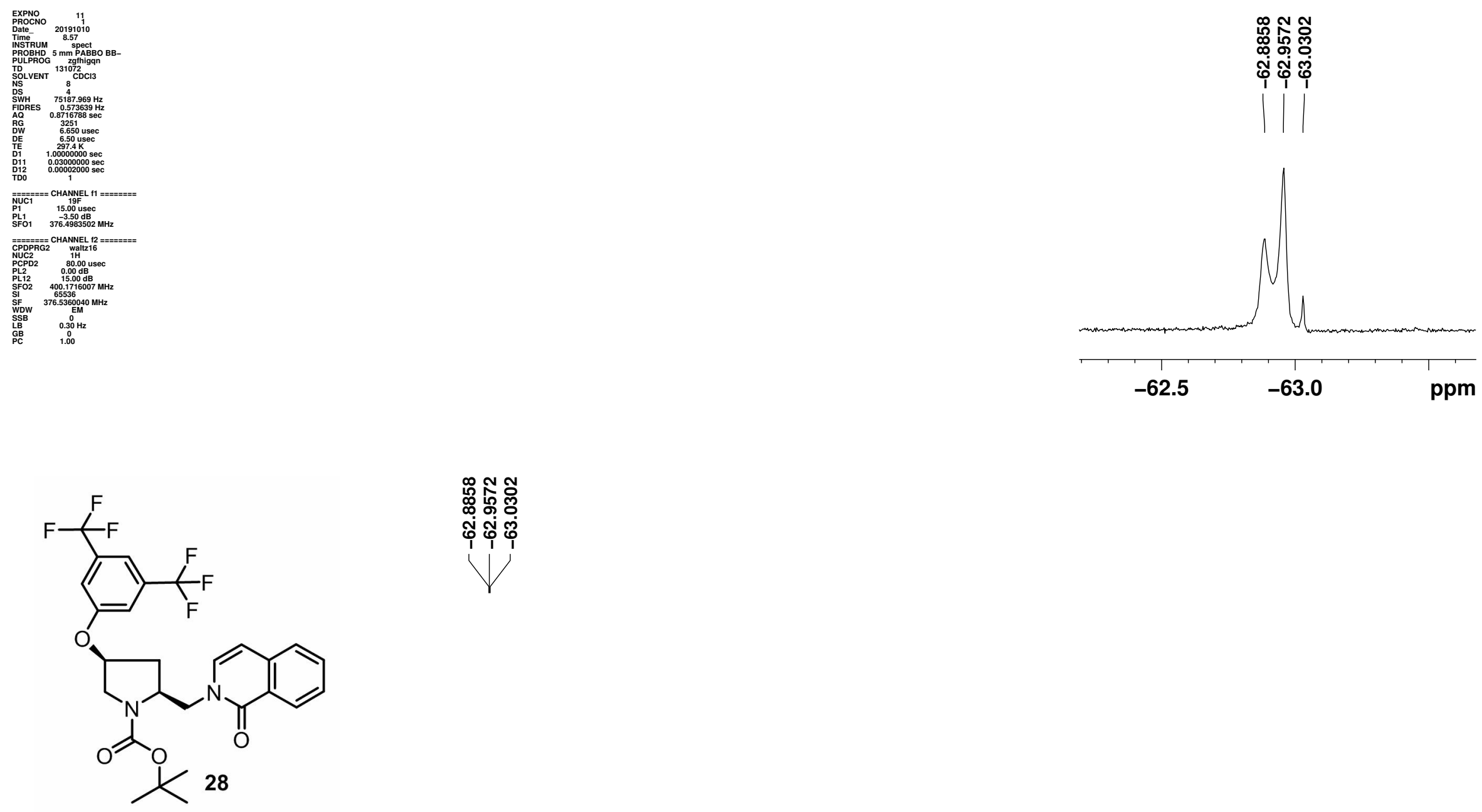

\section{${ }^{19} \mathrm{~F} \mathrm{NMR}, 376 \mathrm{MHz}, \mathrm{CDCl}_{3}$}

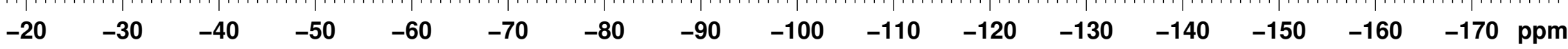



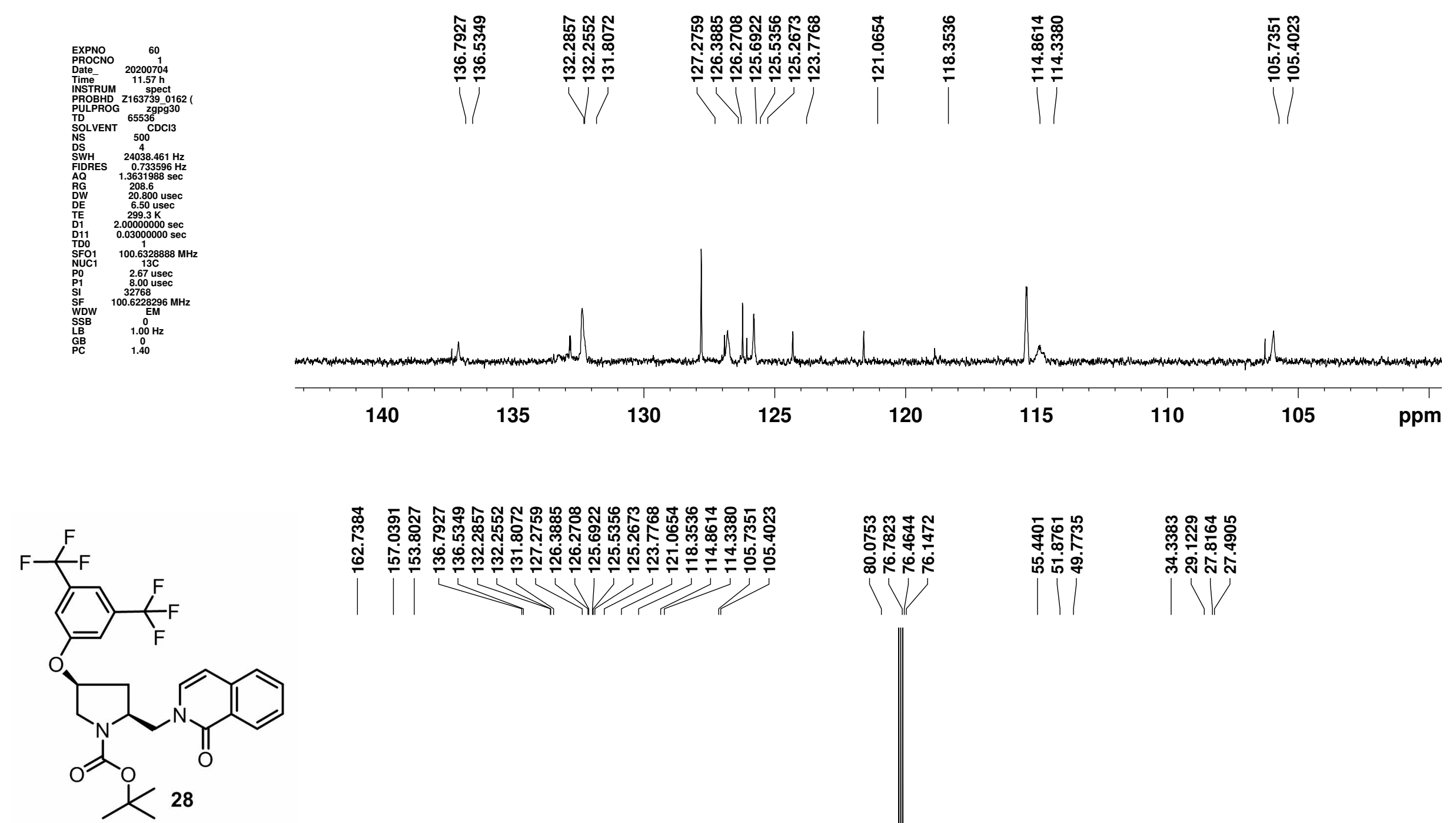

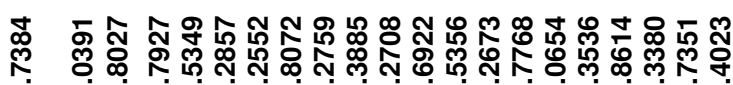

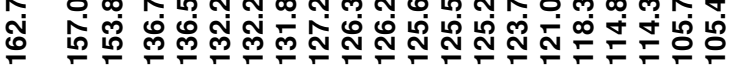

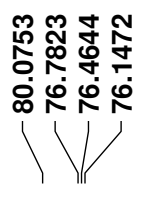

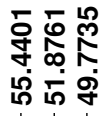

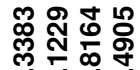

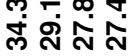

${ }^{13} \mathrm{C}\left\{{ }^{1} \mathrm{H}\right\} \mathrm{NMR}, 100 \mathrm{MHz}, \mathrm{CDCl}_{3}$

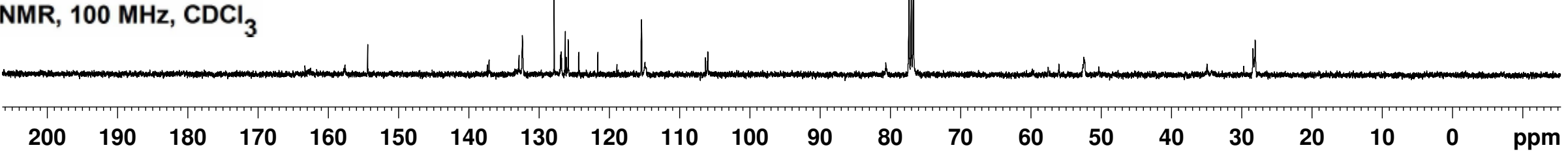



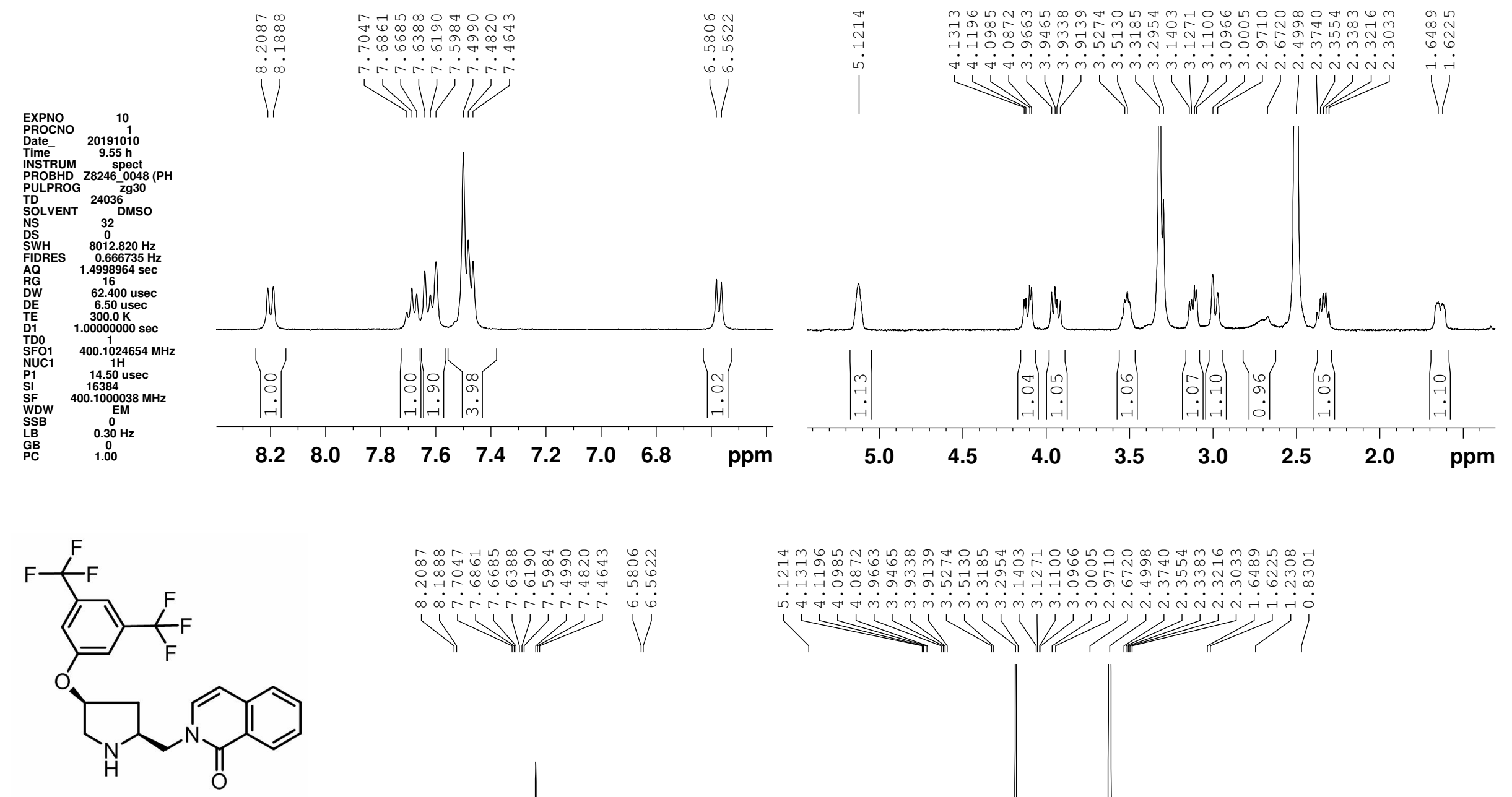

${ }^{1} \mathrm{H} \mathrm{NMR}, 400 \mathrm{MHz}$, DMSO-d 6

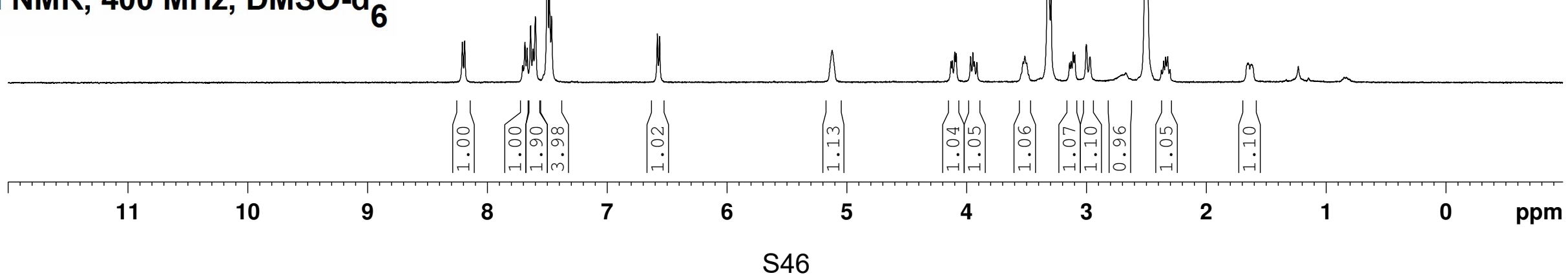



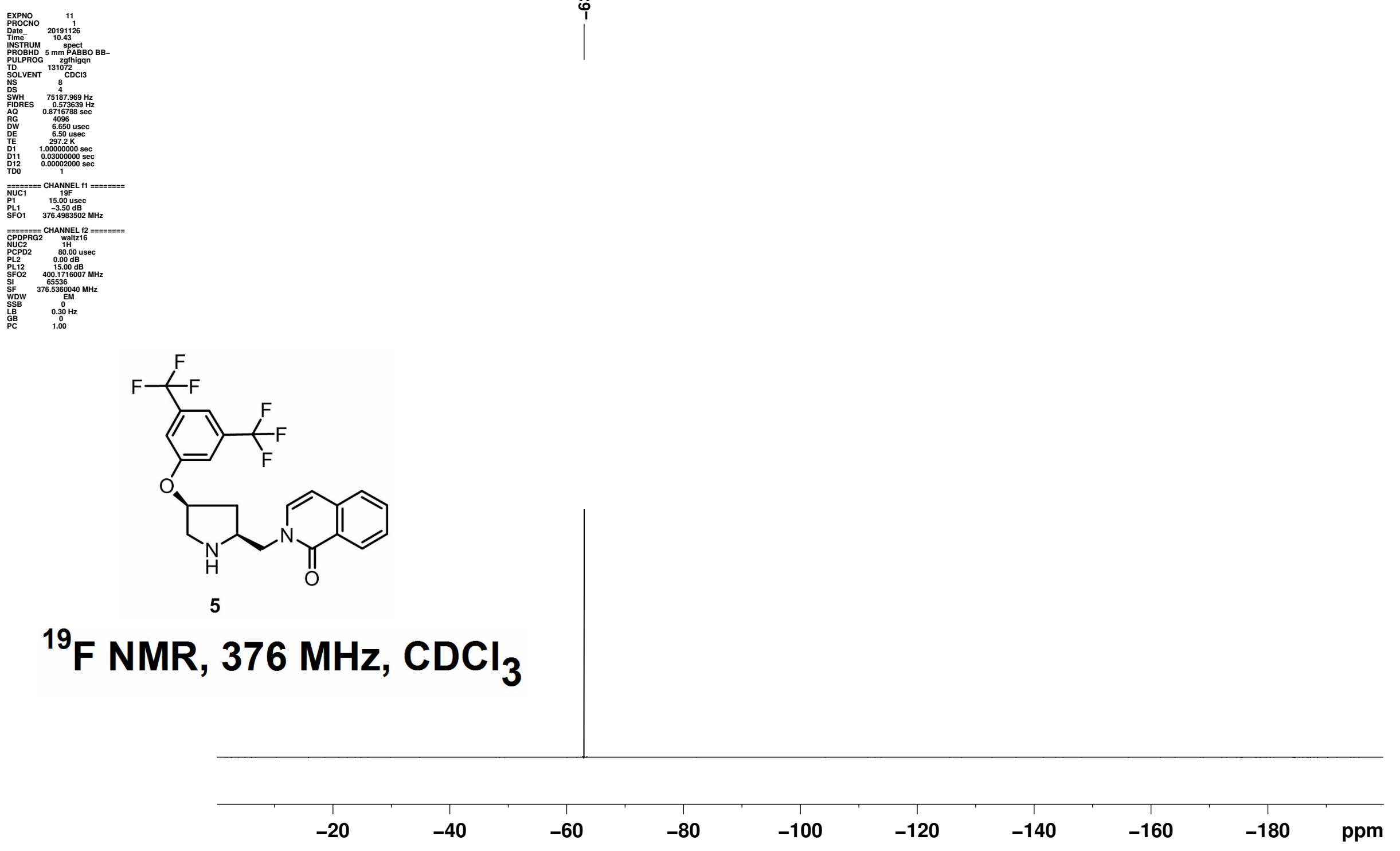

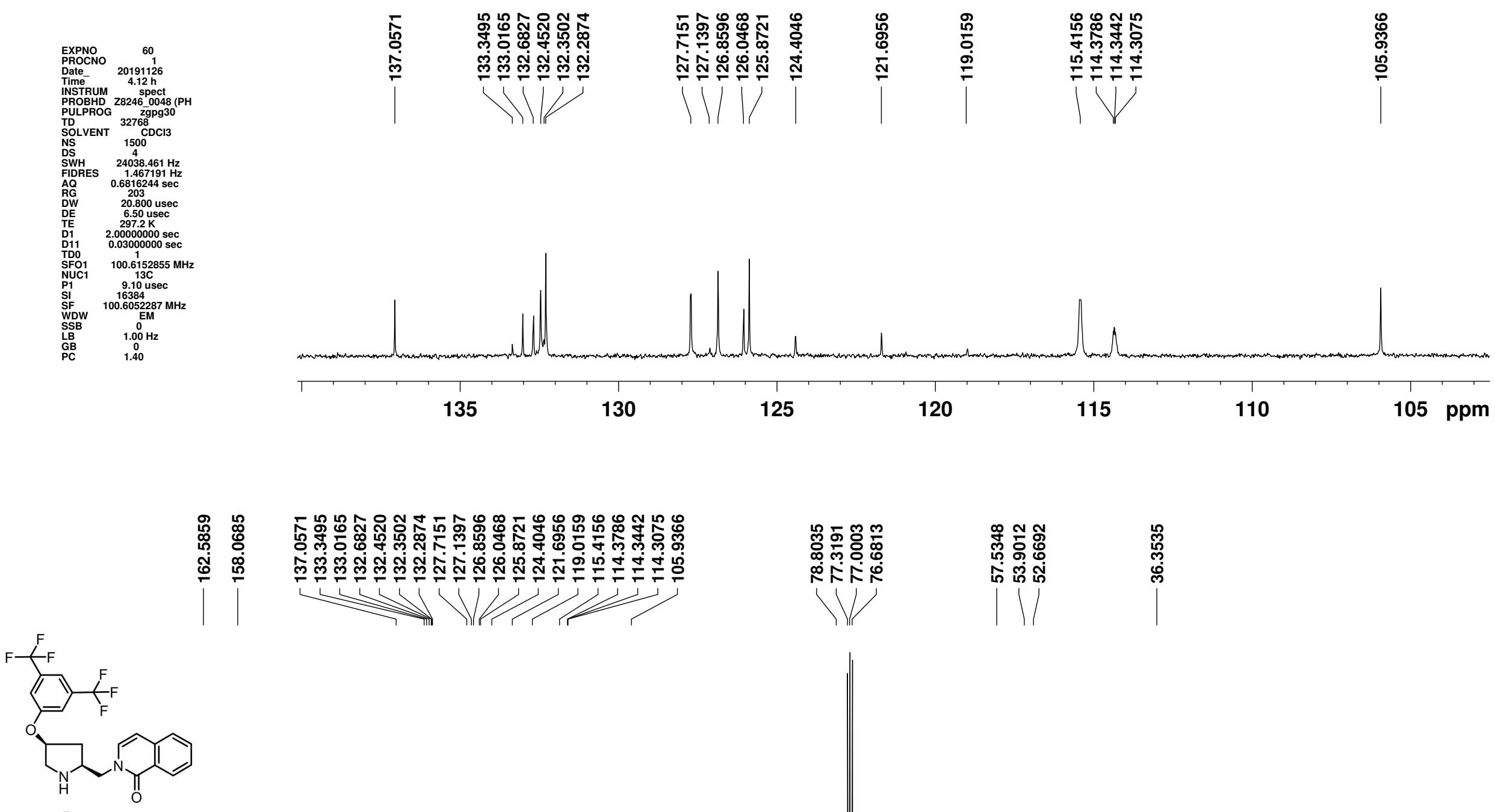

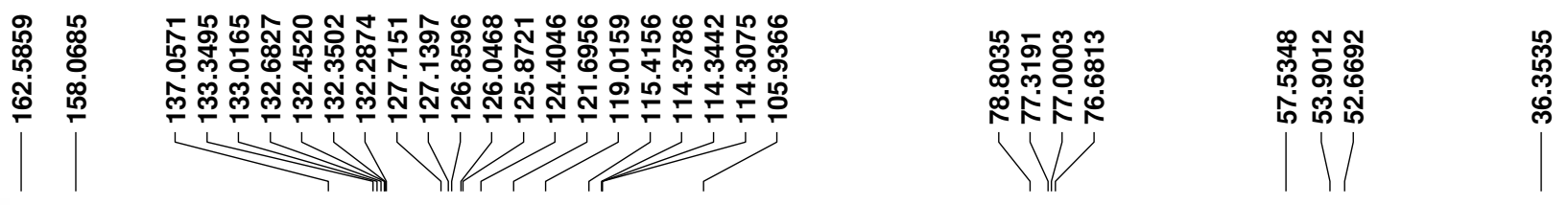

\section{${ }^{13} \mathrm{C}\left\{{ }^{1} \mathrm{H}\right\} \mathrm{NMR}, 100 \mathrm{MHz}, \mathrm{CDCl}_{3}$}
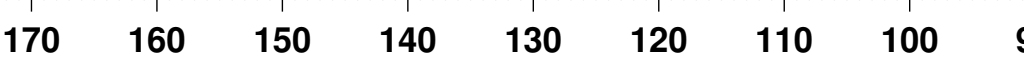

90

80

70

60

50

30

20

$10 \quad 0 \quad \mathrm{ppm}$ 

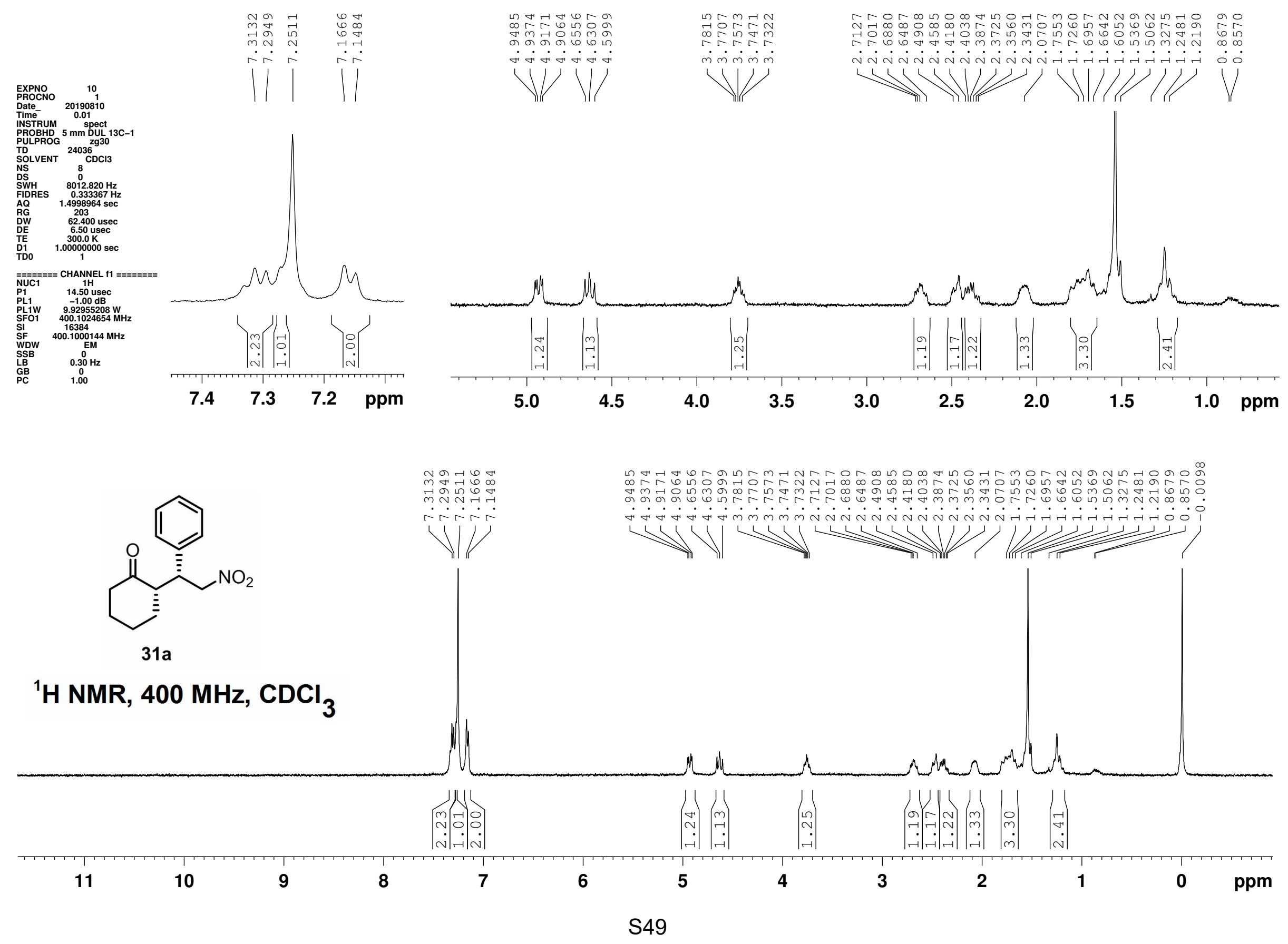

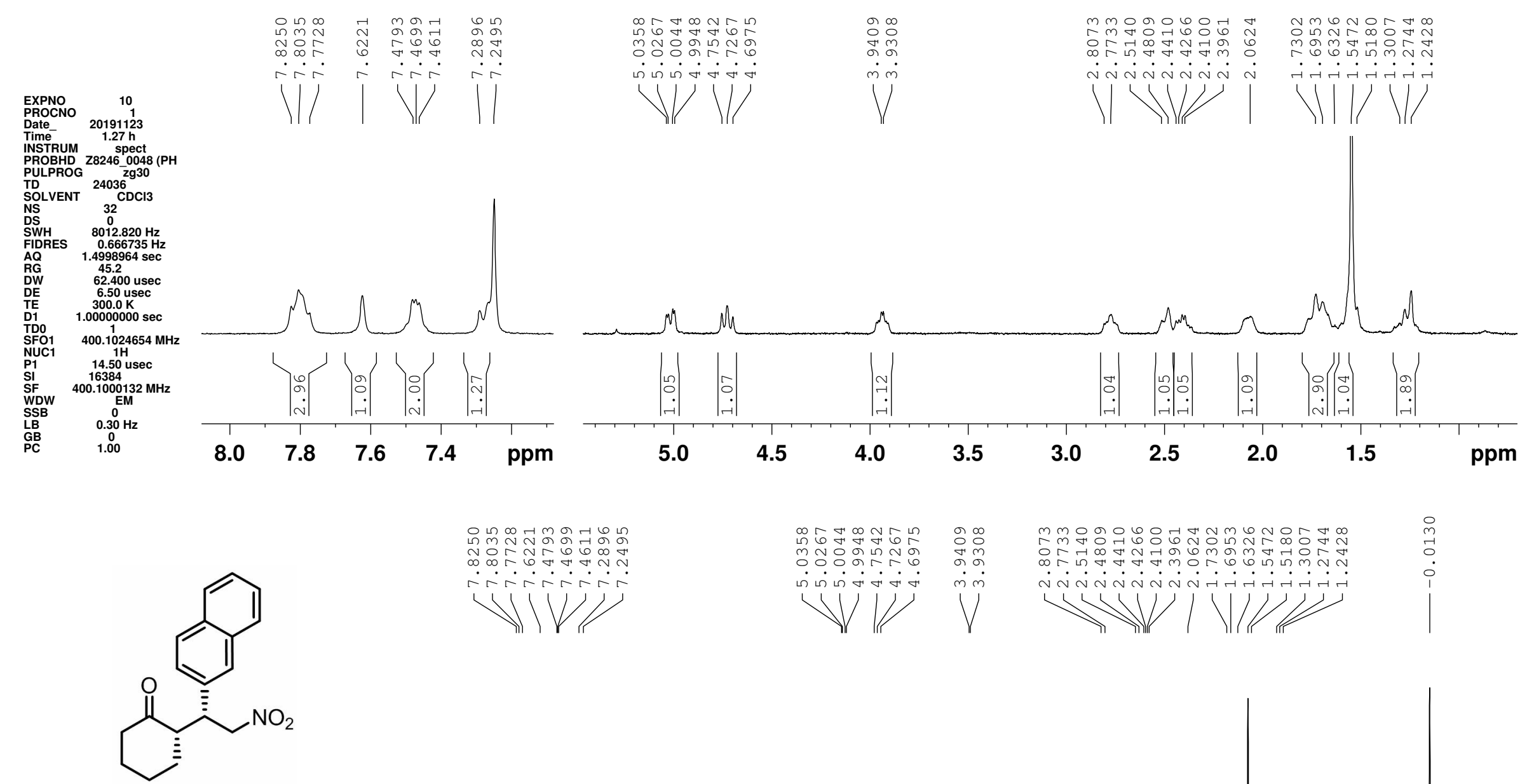

31b

${ }^{1} \mathrm{H}$ NMR, $400 \mathrm{MHz}, \mathrm{CDCl}_{3}$

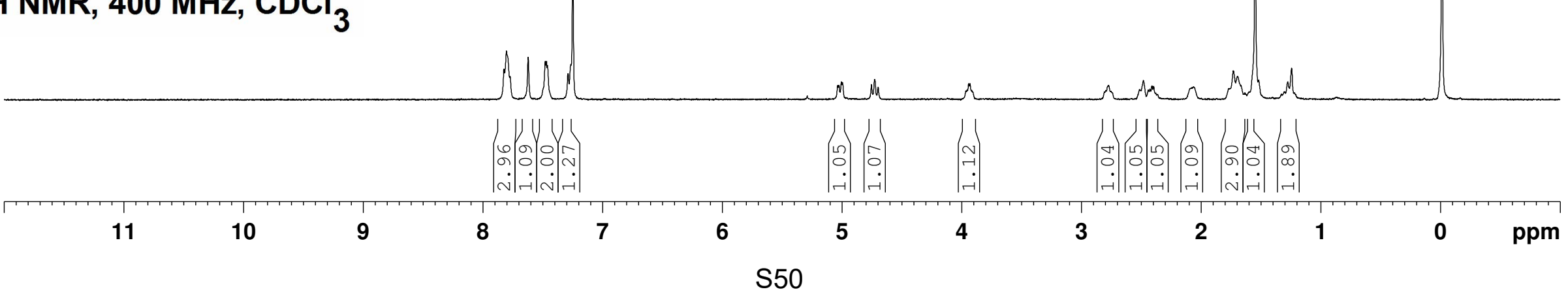



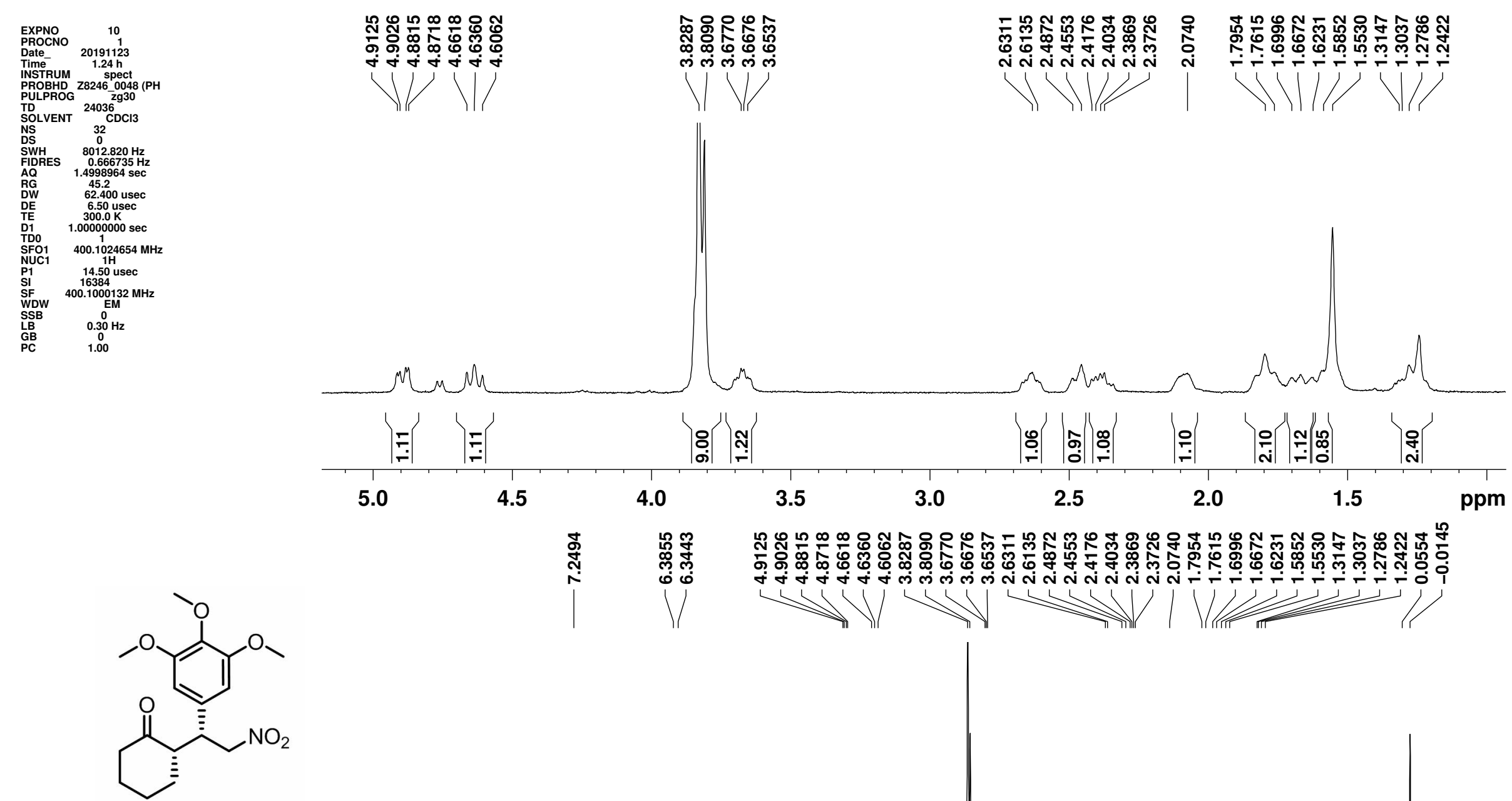

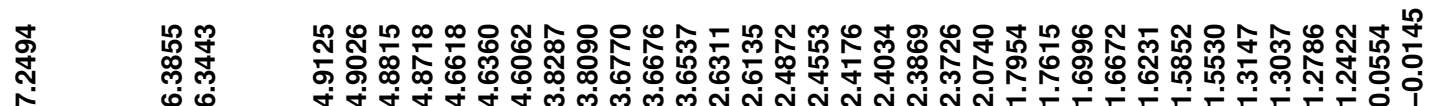

Tุ

$31 \mathrm{c}$

${ }^{1} \mathrm{H}$ NMR, $400 \mathrm{MHz}, \mathrm{CDCl}_{3}$

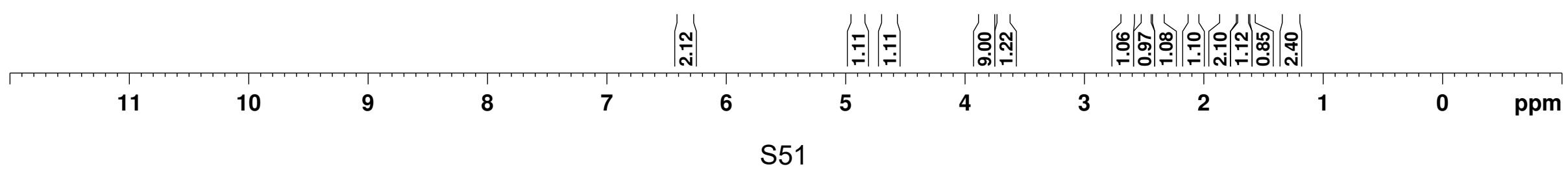



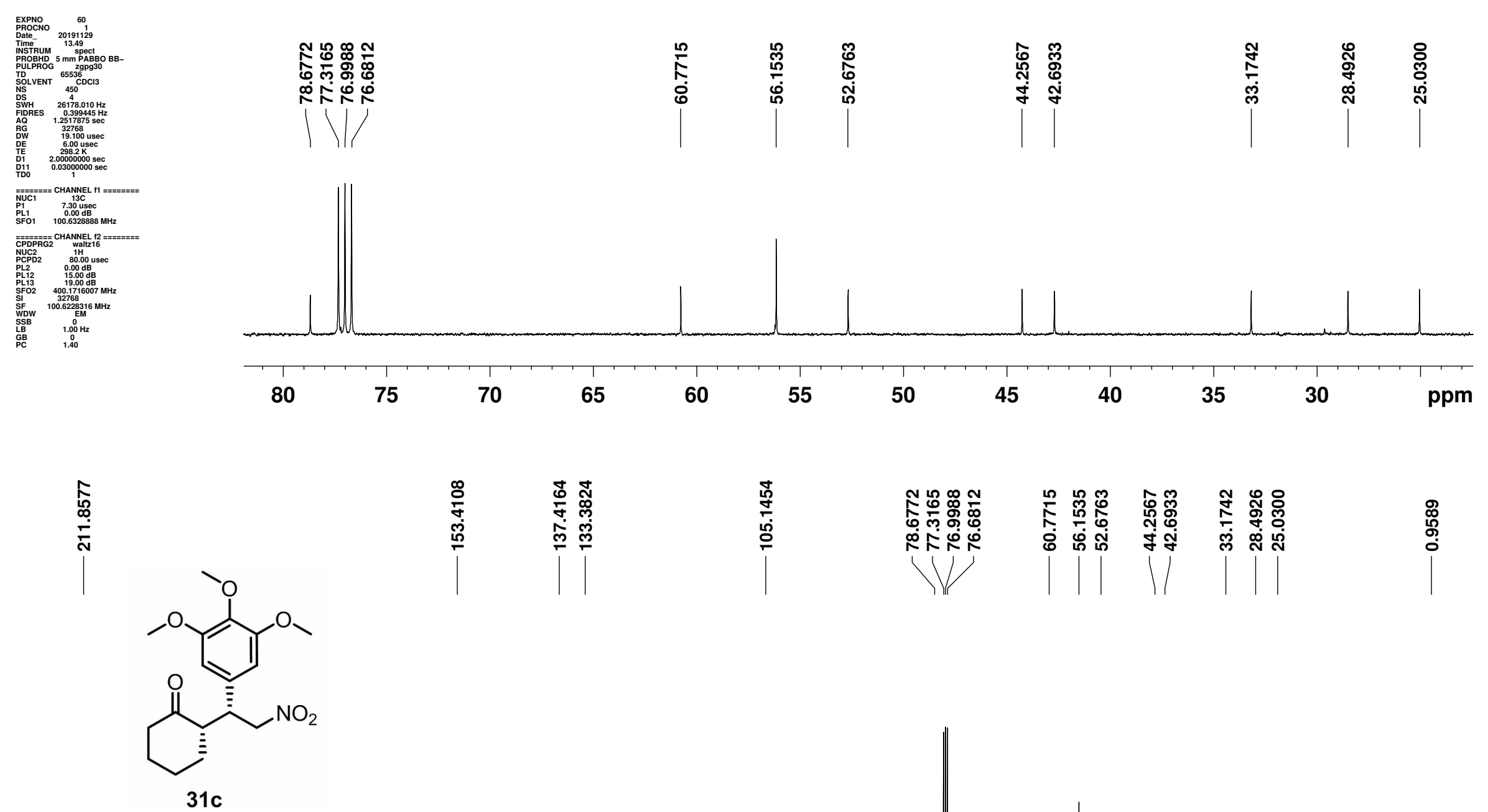

${ }^{13} \mathrm{C}\left\{{ }^{1} \mathrm{H}\right\}$ NMR, $100 \mathrm{MHz}, \mathrm{CDCl}_{3}$

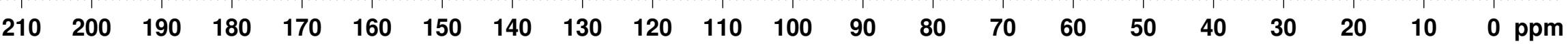



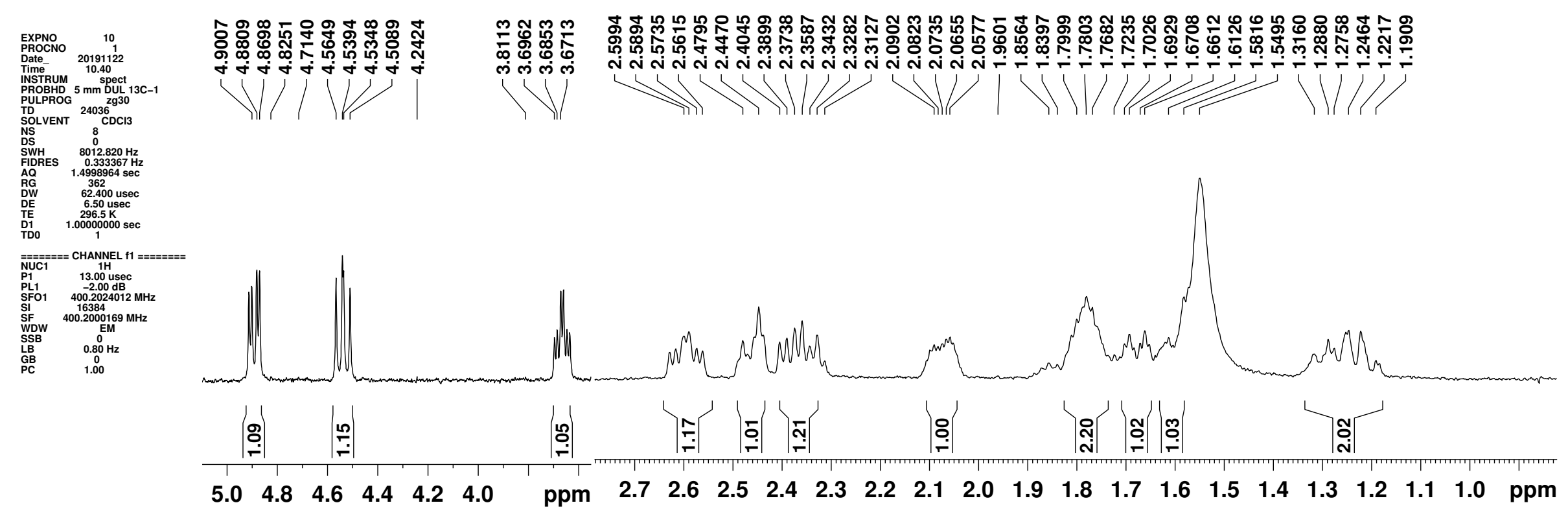

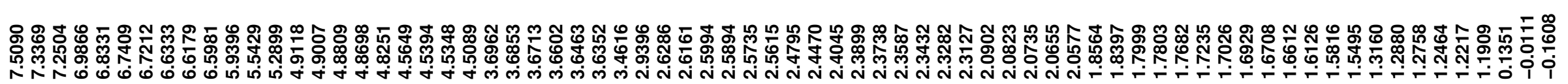<smiles>O=C1CCCCC1[C@H](C[N+](=O)[O-])c1ccc2c(c1)OCO2</smiles>

31d

${ }^{1} \mathrm{H}$ NMR, $400 \mathrm{MHz}, \mathrm{CDCl}_{3}$

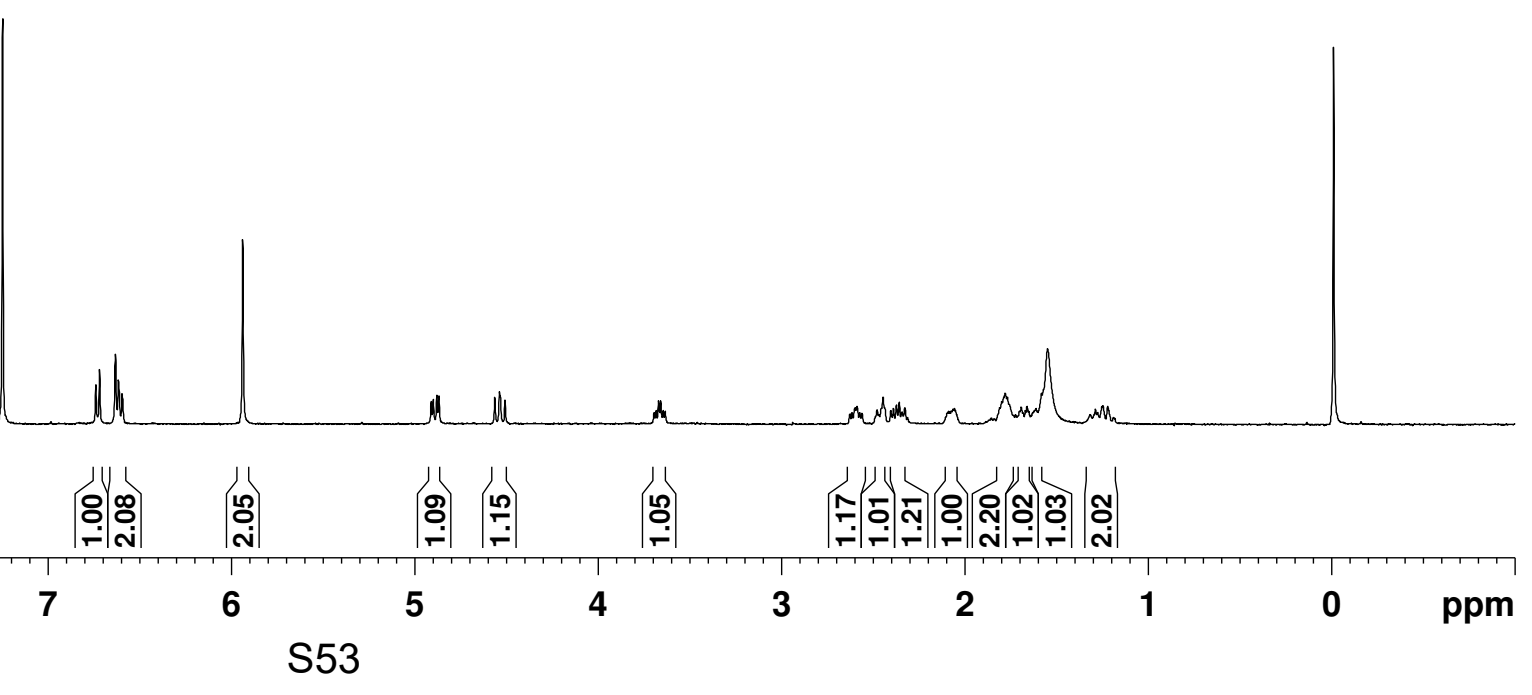



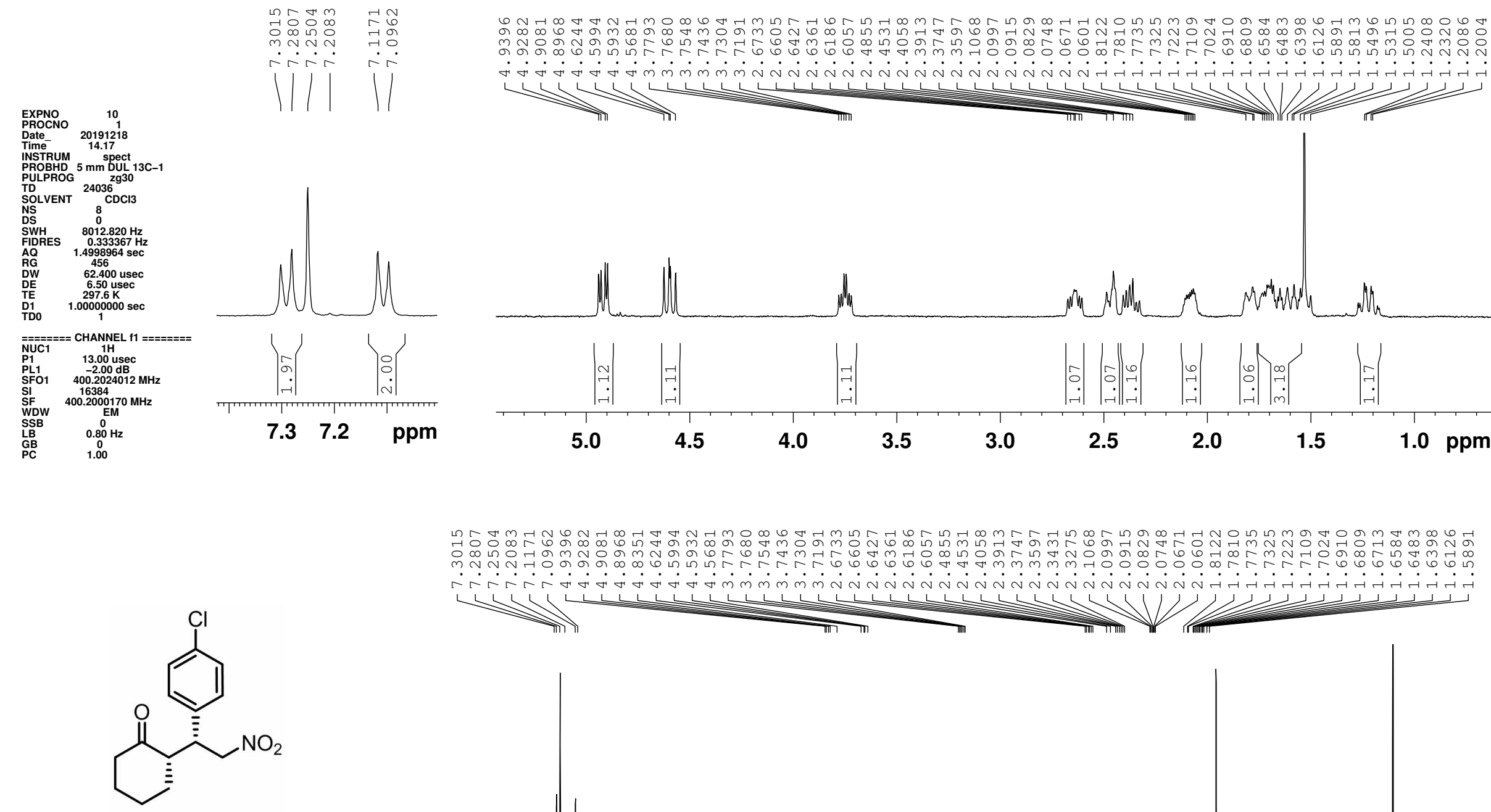

$31 \mathrm{e}$

${ }^{1} \mathrm{H}$ NMR, $400 \mathrm{MHz}, \mathrm{CDCl}_{3}$
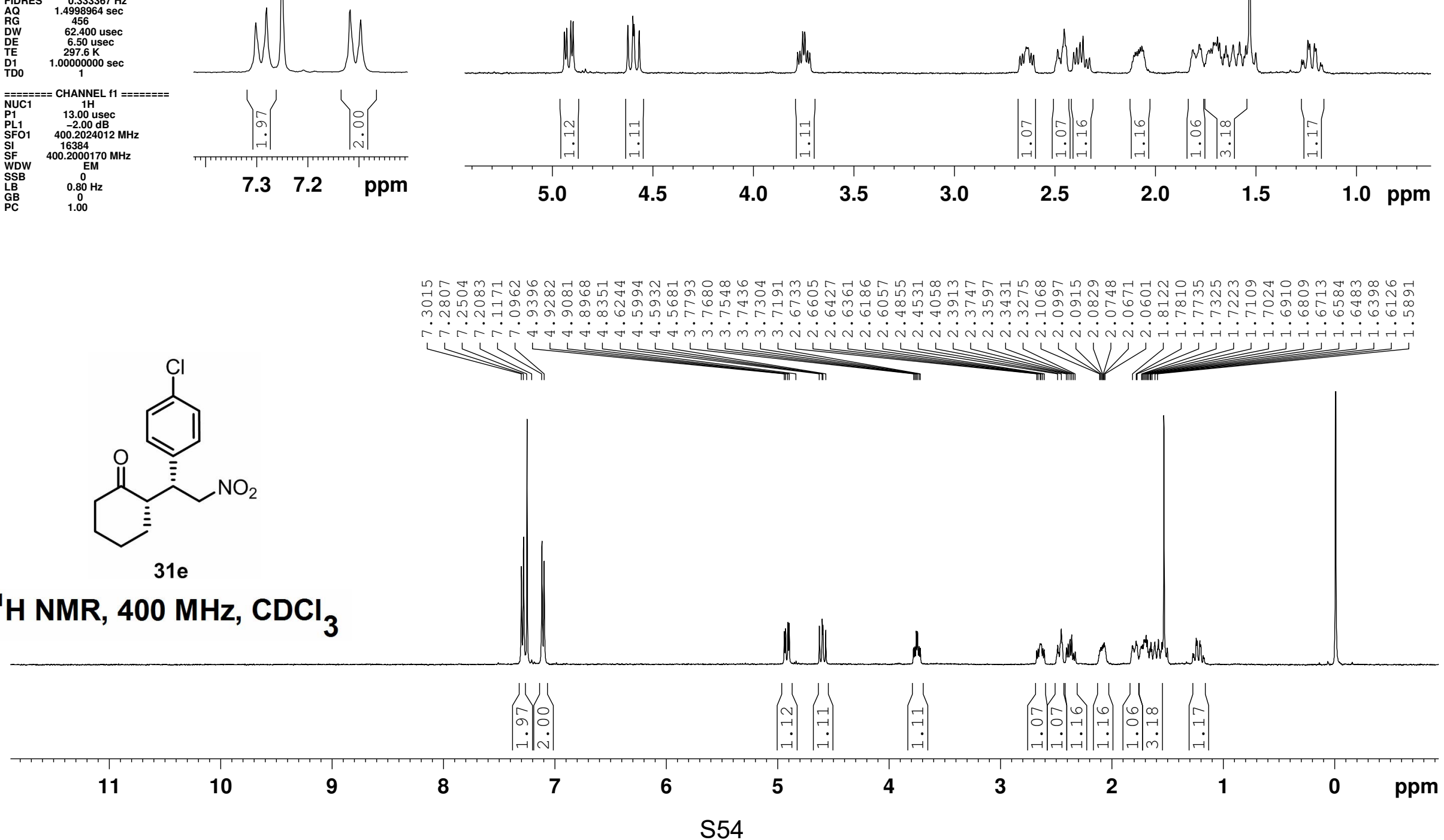

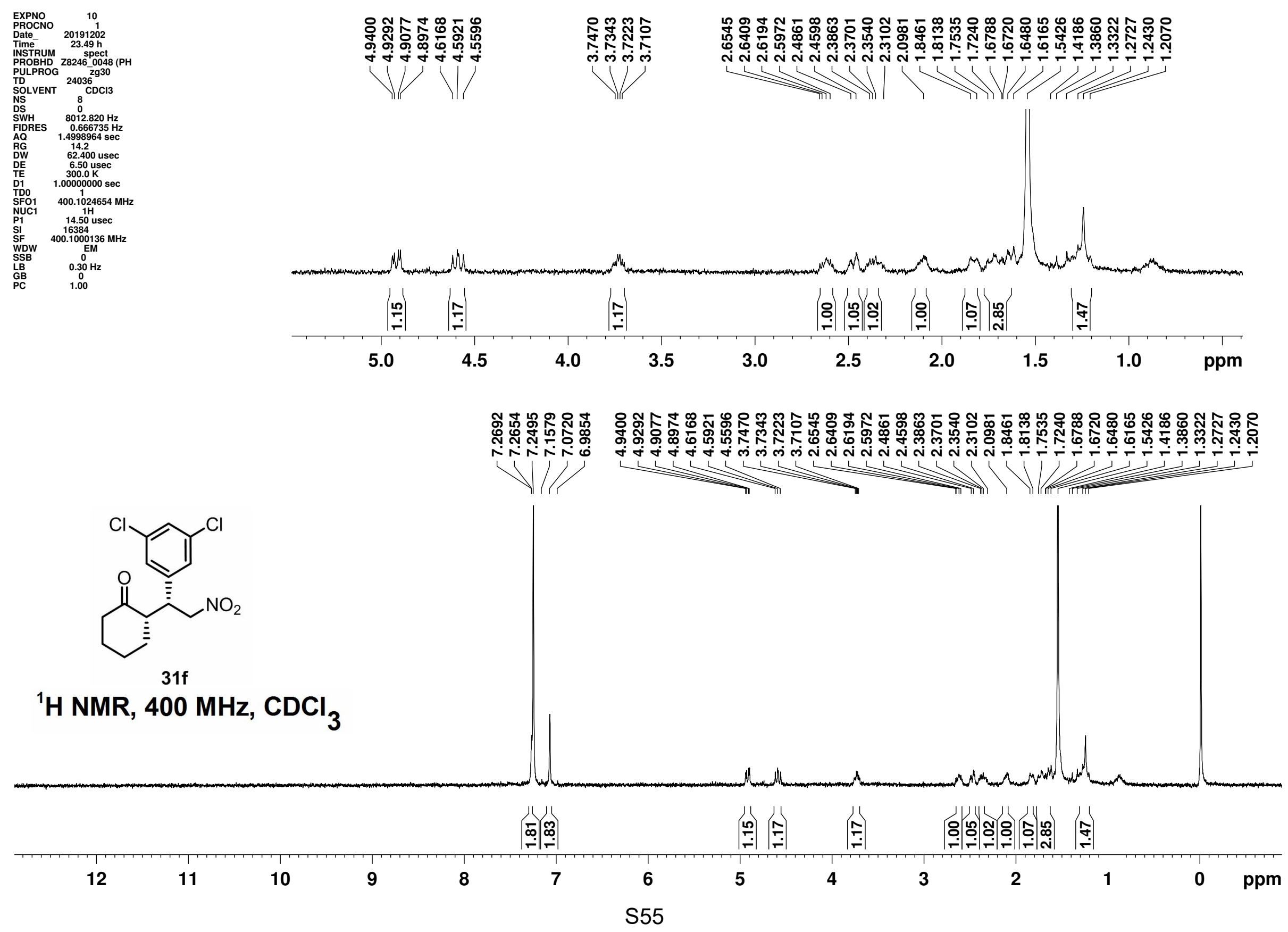

$31 \mathrm{f}$

${ }^{1} \mathrm{H}$ NMR, $400 \mathrm{MHz}, \mathrm{CDCl}_{3}$

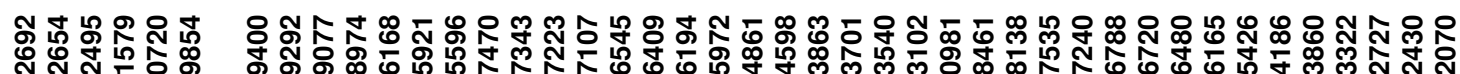

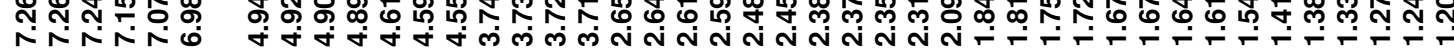



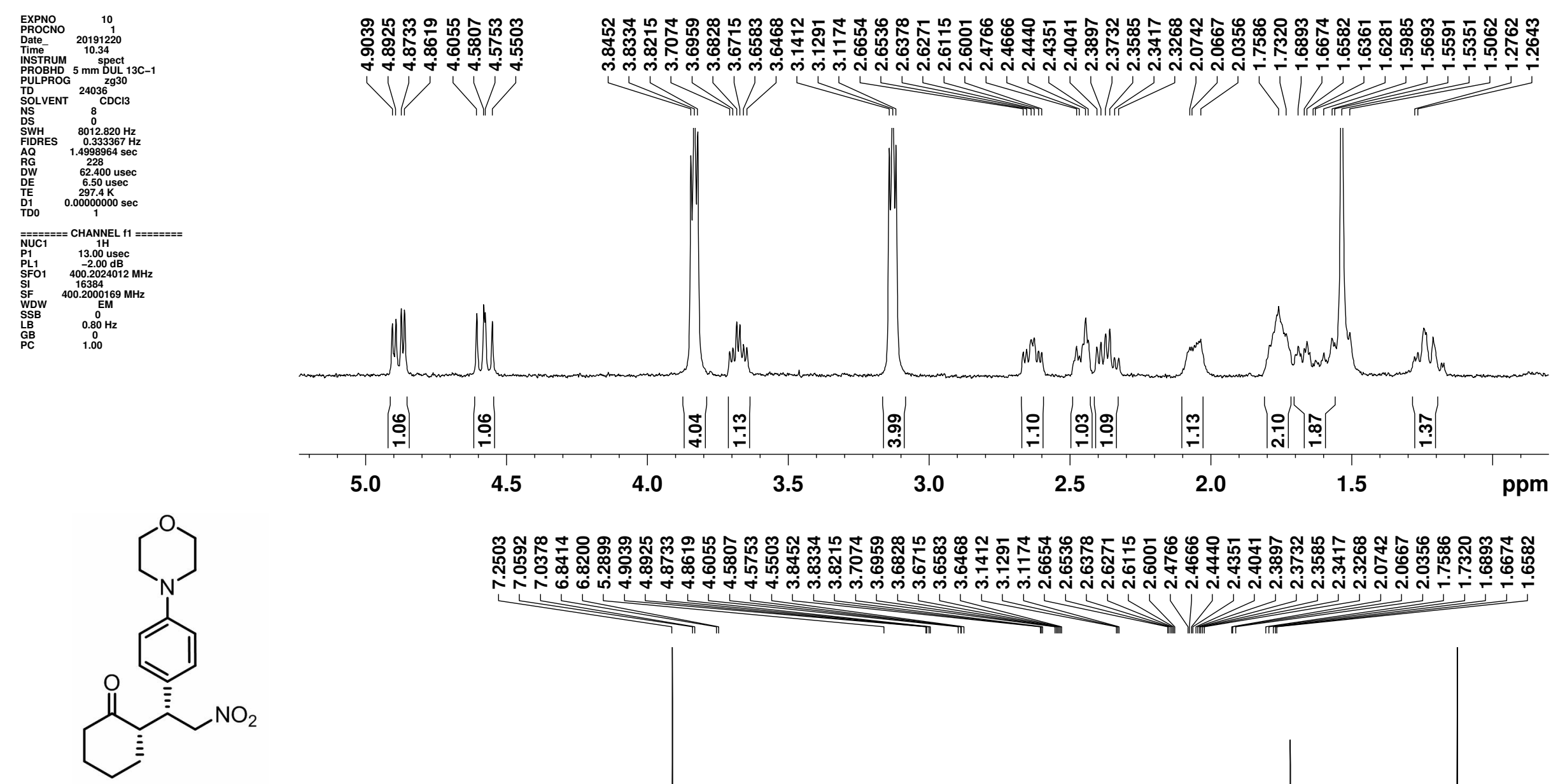

$31 \mathrm{~g}$

${ }^{1} \mathrm{H}$ NMR, $400 \mathrm{MHz}, \mathrm{CDCl}_{3}$

\begin{tabular}{|c|c|c|c|c|c|c|c|c|c|c|c|c|c|c|}
\hline & & & & & & ஸ̣̂ & & $\left|\begin{array}{l}0 \\
\hdashline \\
\dot{1}\end{array}\right|$ & $\left|\begin{array}{l}\mid \\
0\end{array}\right|$ & $\left|\begin{array}{c}\mathbf{D} \\
\text { ஸे }\end{array}\right|$ & 害| & 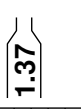 & & \\
\hline 13 & 12 & 11 & 10 & 9 & 8 & 7 & 6 & 5 & 4 & 3 & 2 & 1 & 0 & ppm \\
\hline
\end{tabular}



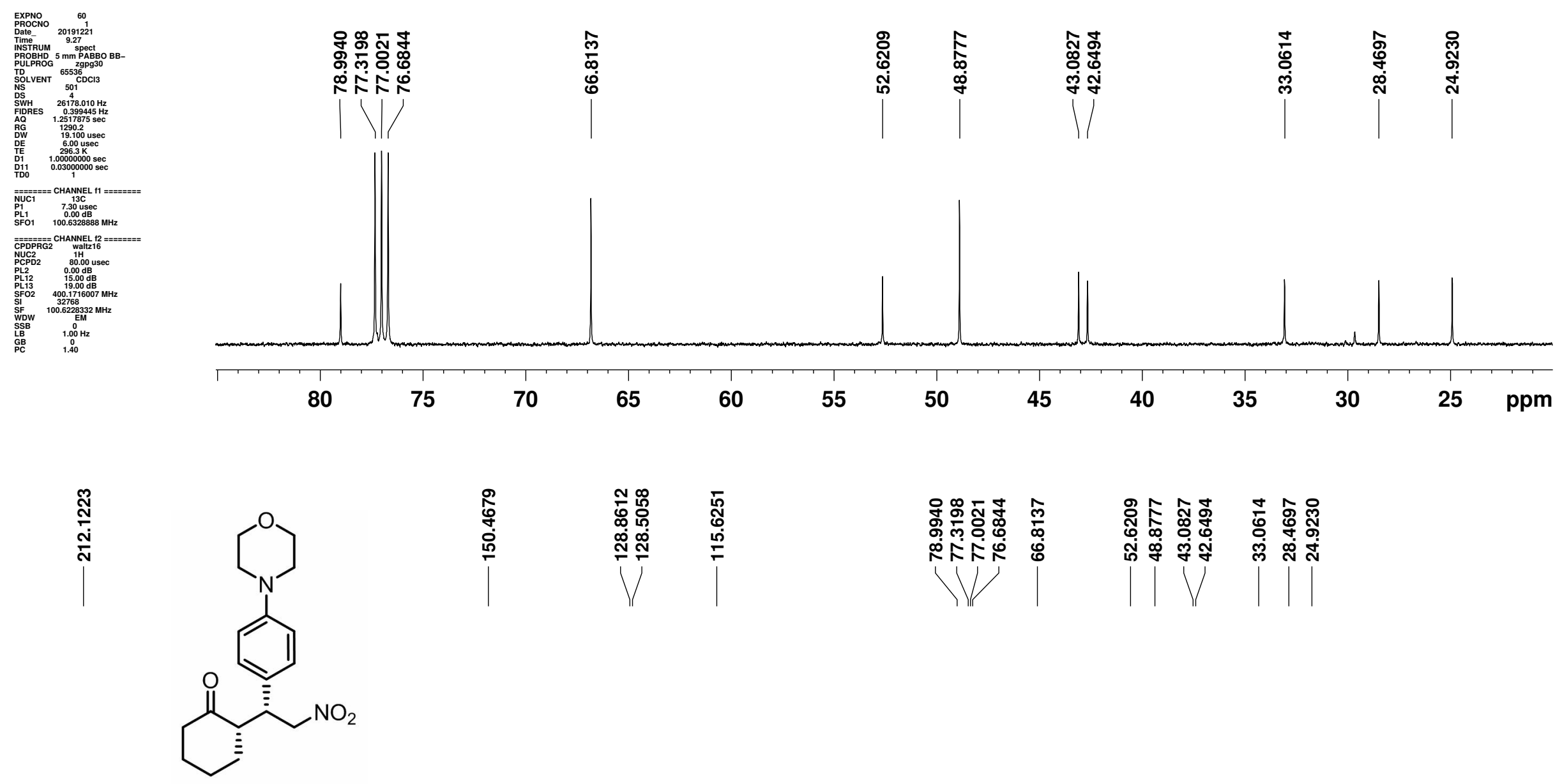

$31 \mathrm{~g}$

${ }^{13} \mathrm{C}\left\{{ }^{1} \mathrm{H}\right\} \mathrm{NMR}, 100 \mathrm{MHz}, \mathrm{CDCl}_{3}$

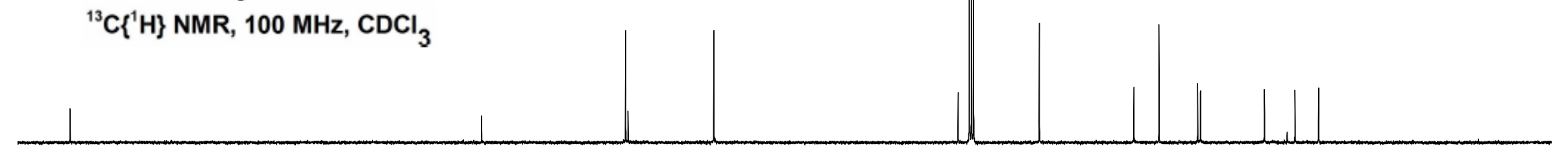

$\begin{array}{lllllllllllllllllllllll}210 & 200 & 190 & 180 & 170 & 160 & 150 & 140 & 130 & 120 & 110 & 100 & 90 & 80 & 70 & 60 & 50 & 40 & 30 & 20 & 10 & 0 & \mathrm{ppm}\end{array}$ 

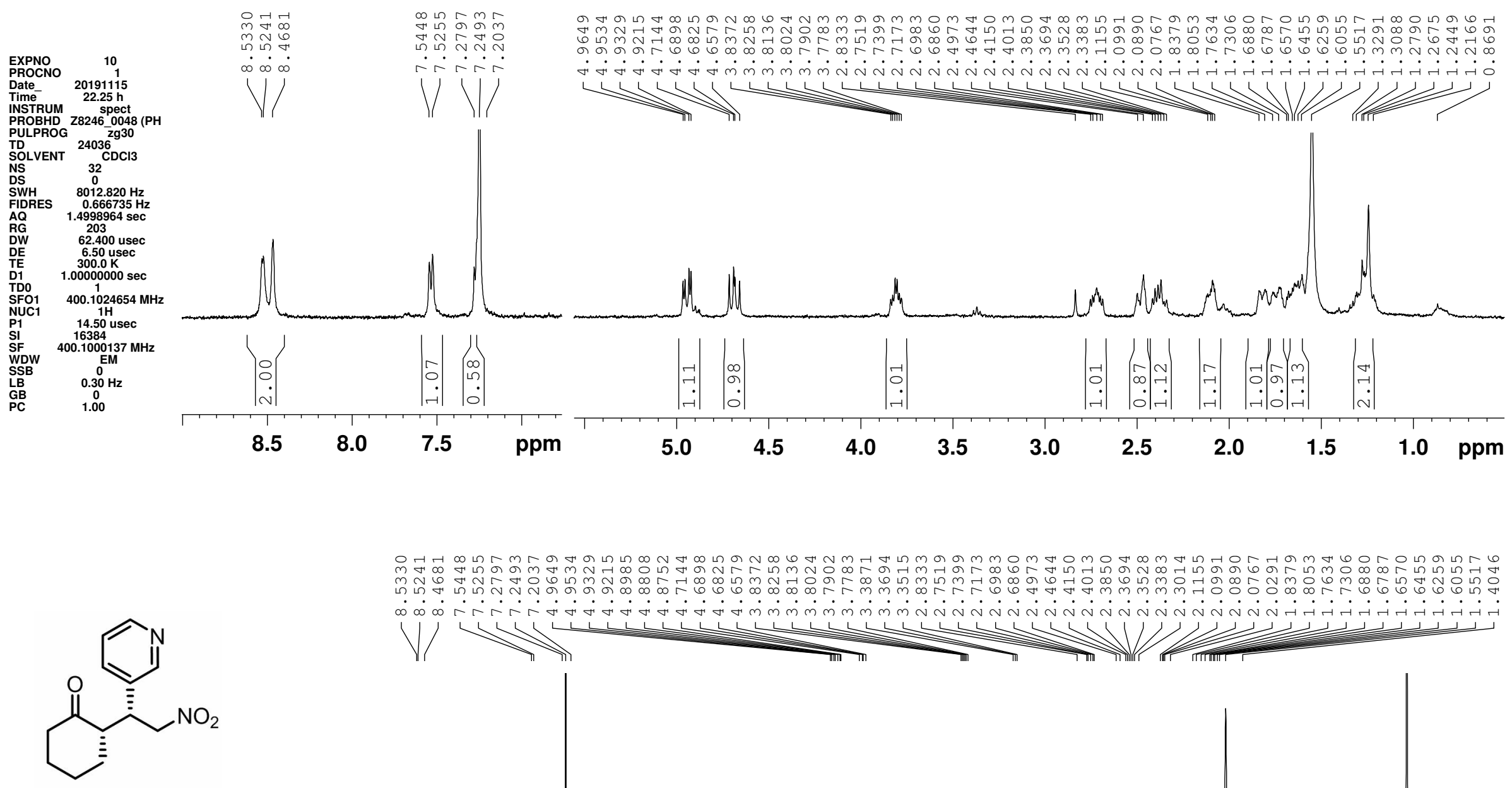

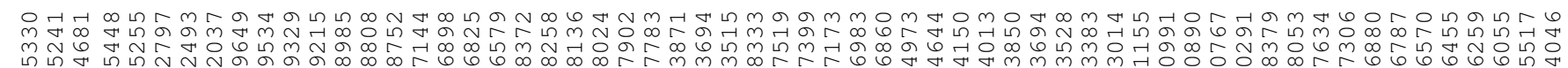

$31 \mathrm{~h}$

${ }^{1} \mathrm{H} \mathrm{NMR}, 400 \mathrm{MHz}, \mathrm{CDCl}_{3}$

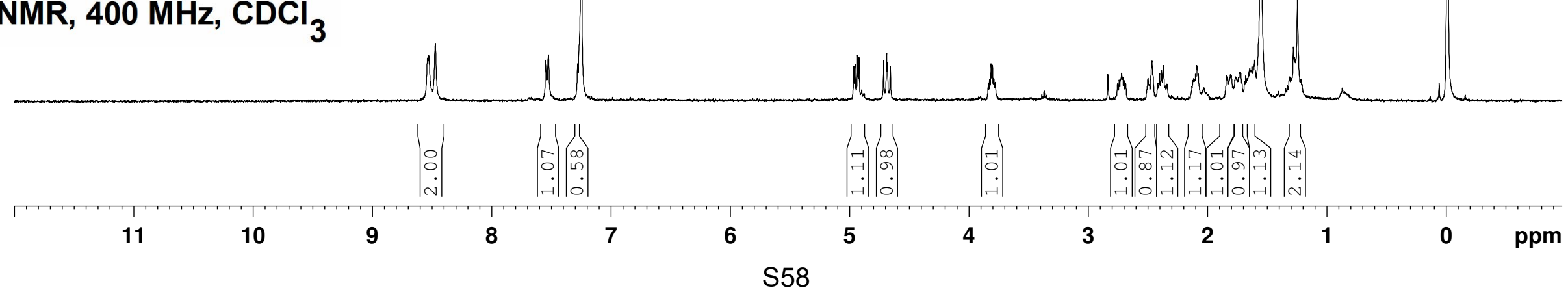



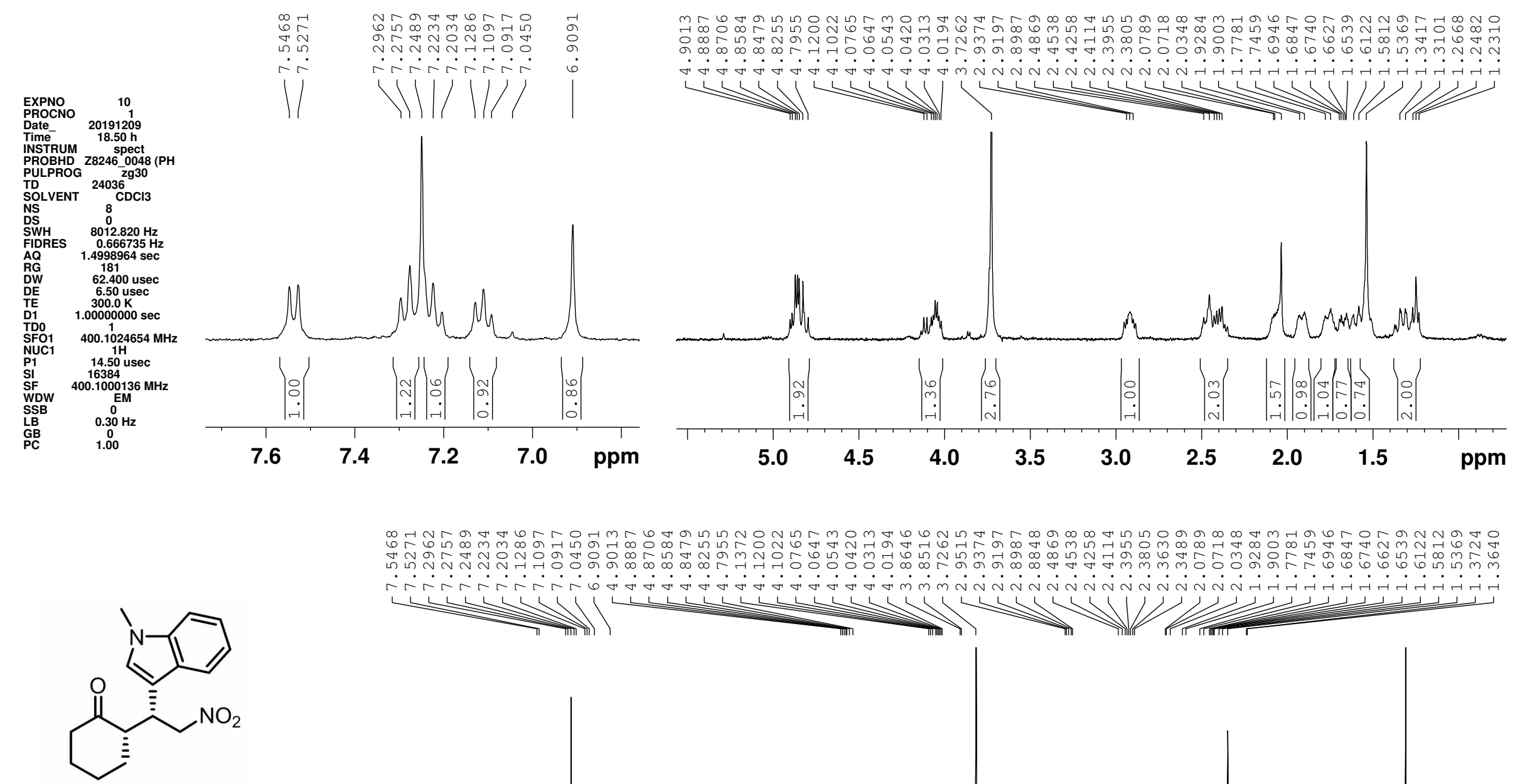

$31 i$

${ }^{1} \mathrm{H}$ NMR, $400 \mathrm{MHz}, \mathrm{CDCl}_{3}$
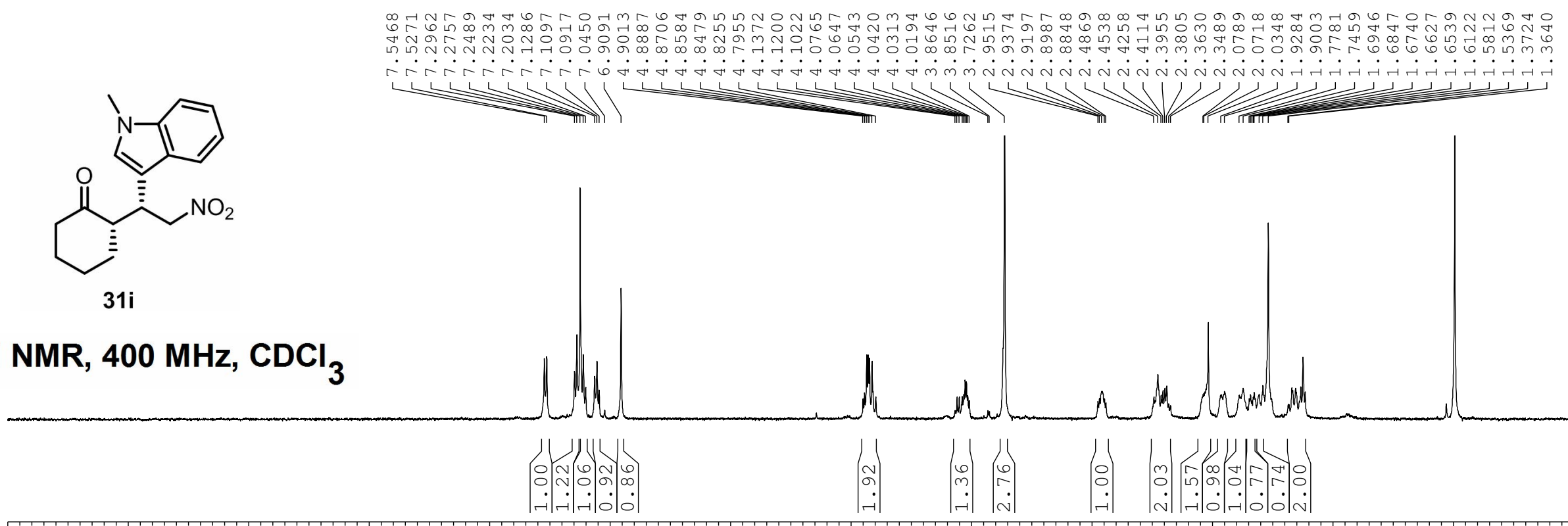

$11 \quad 10$

9

8

7

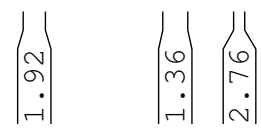

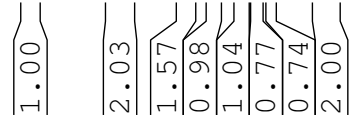

4

32

2

1

0 ppm 

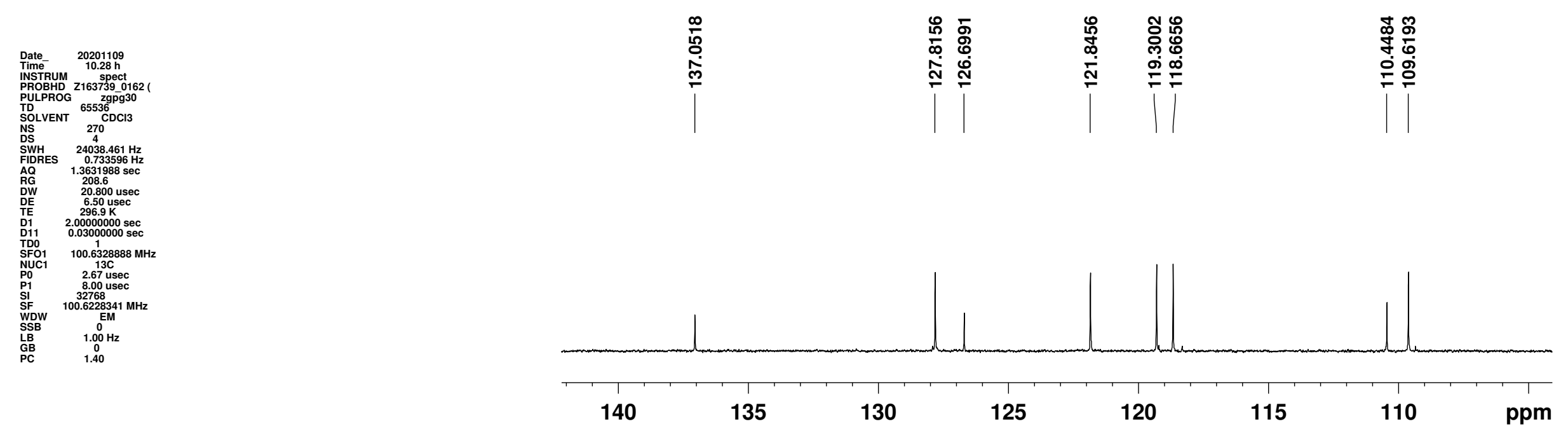

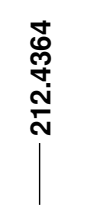
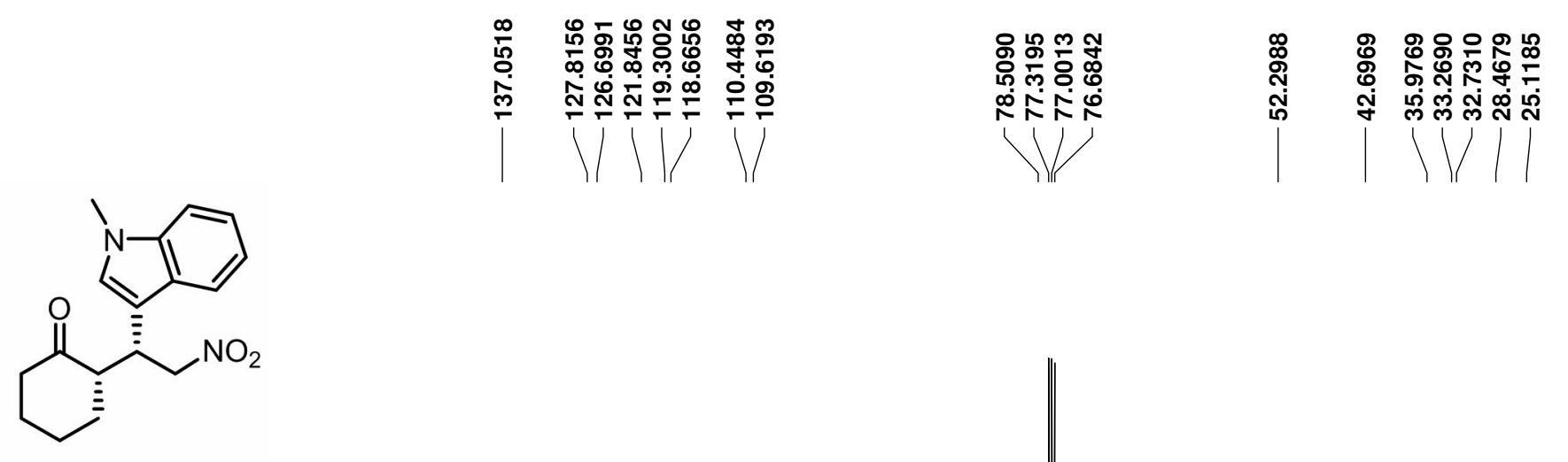

$31 i$

${ }^{13} \mathrm{C}\left\{{ }^{1} \mathrm{H}\right\} \mathrm{NMR}, 100 \mathrm{MHz}, \mathrm{CDCl}_{3}$

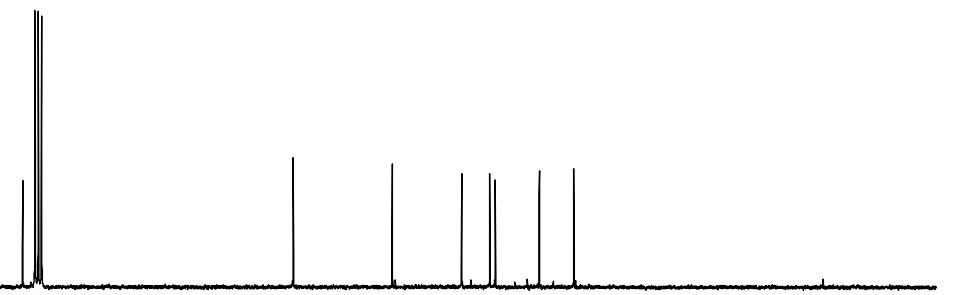

2102

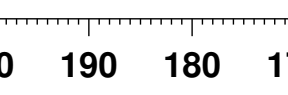

$170-160-150$

130

120

110100

90

80

70

60

50

$40 \quad 30$

20

$10 \quad 0 \mathrm{ppm}$ 


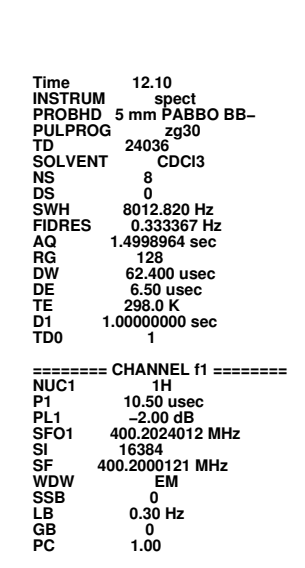

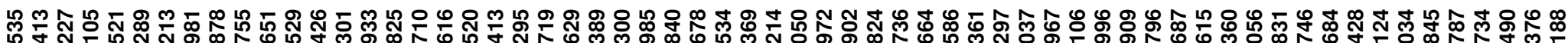

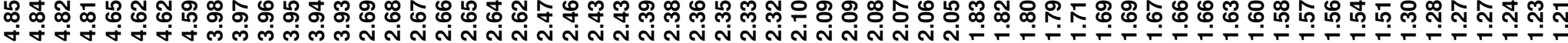

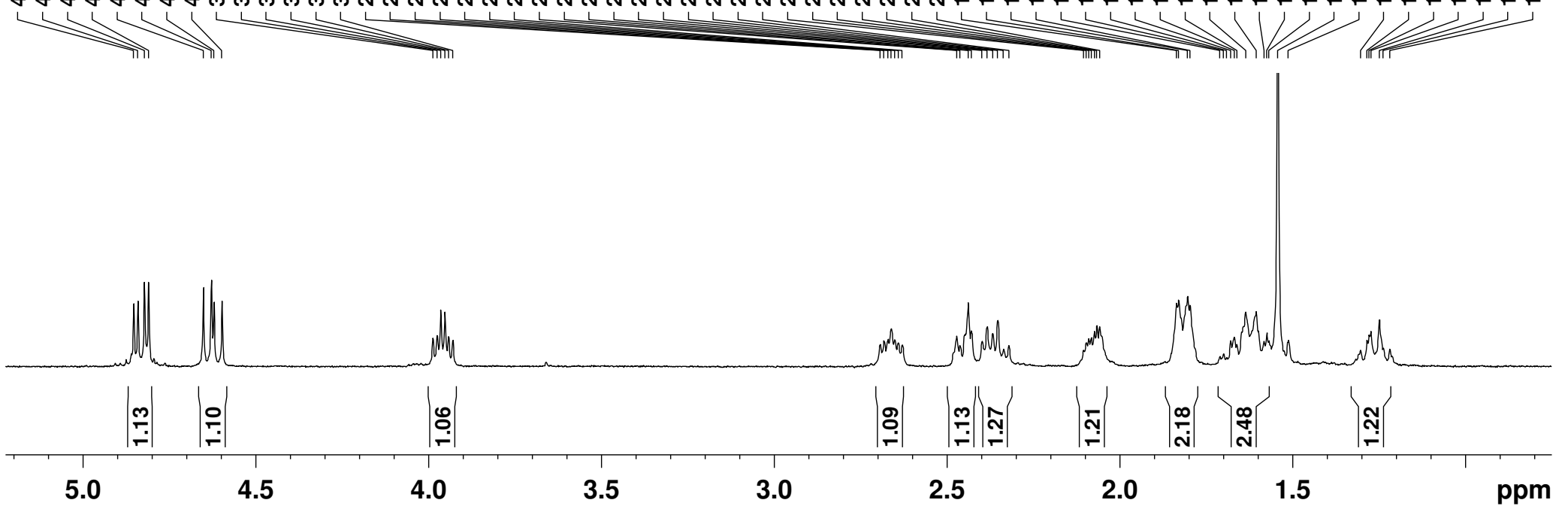

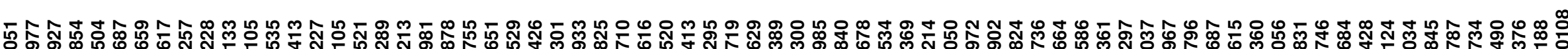
F⿻上丨

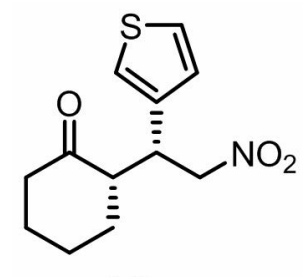

31j

${ }^{1} \mathrm{H}$ NMR, $400 \mathrm{MHz}, \mathrm{CDCl}_{3}$

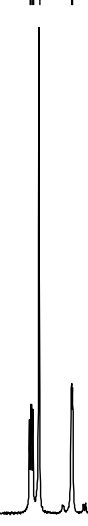

ชِ 

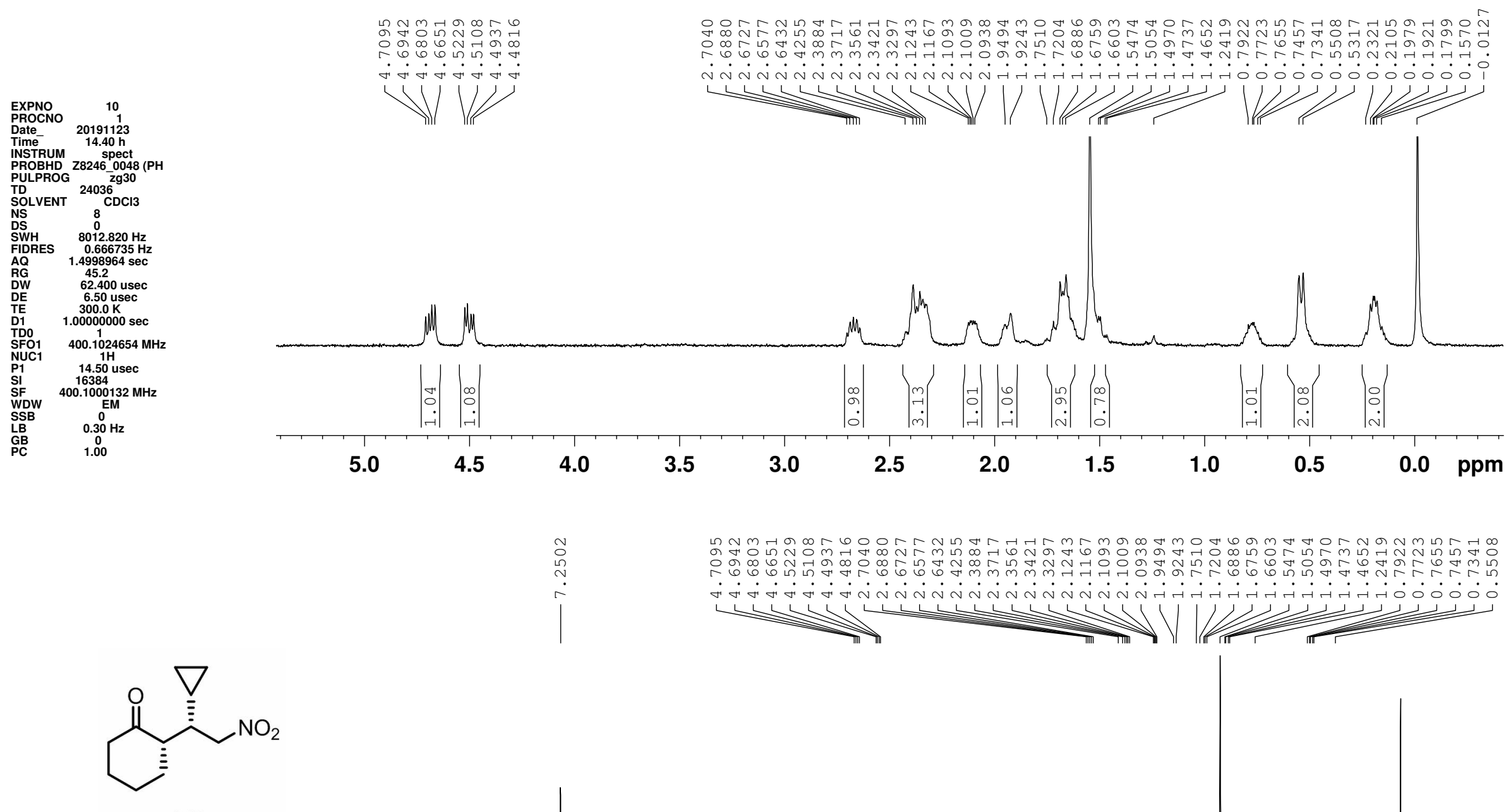

$31 \mathrm{k}$

${ }^{1} \mathrm{H}$ NMR, $400 \mathrm{MHz}, \mathrm{CDCl}_{3}$
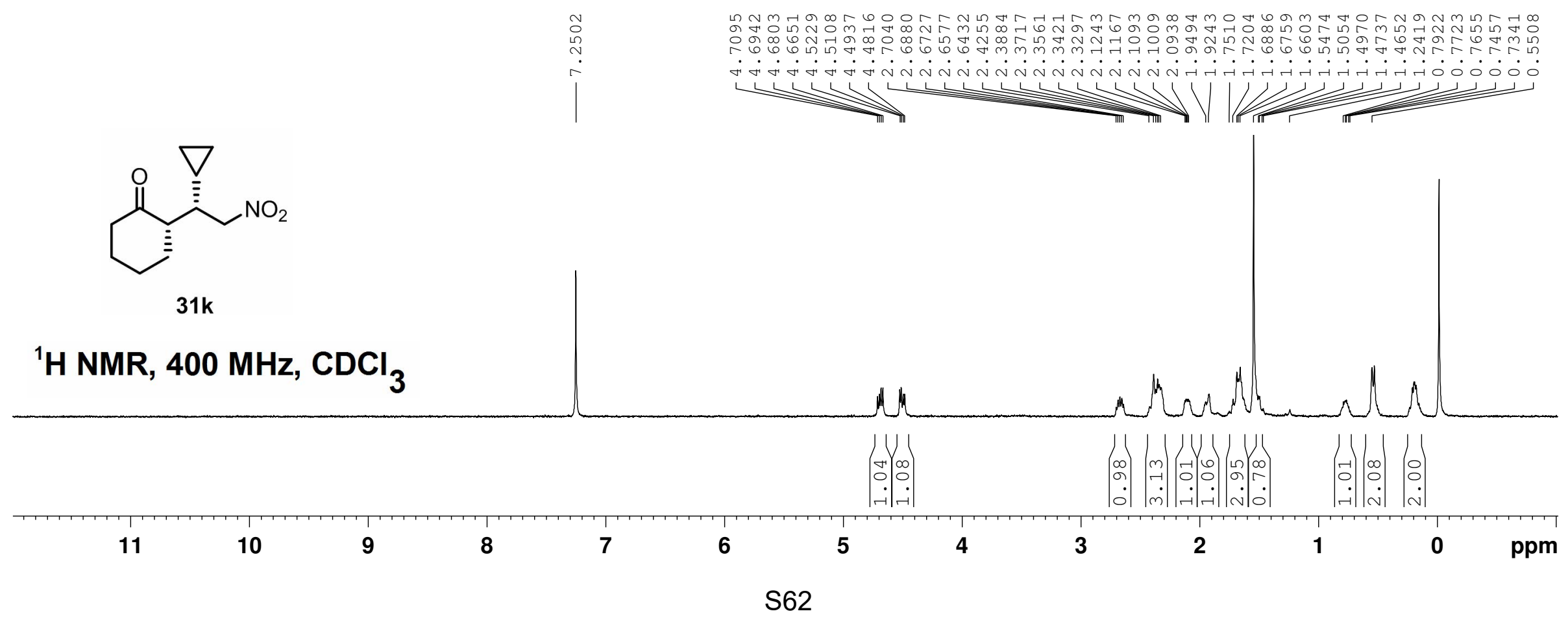

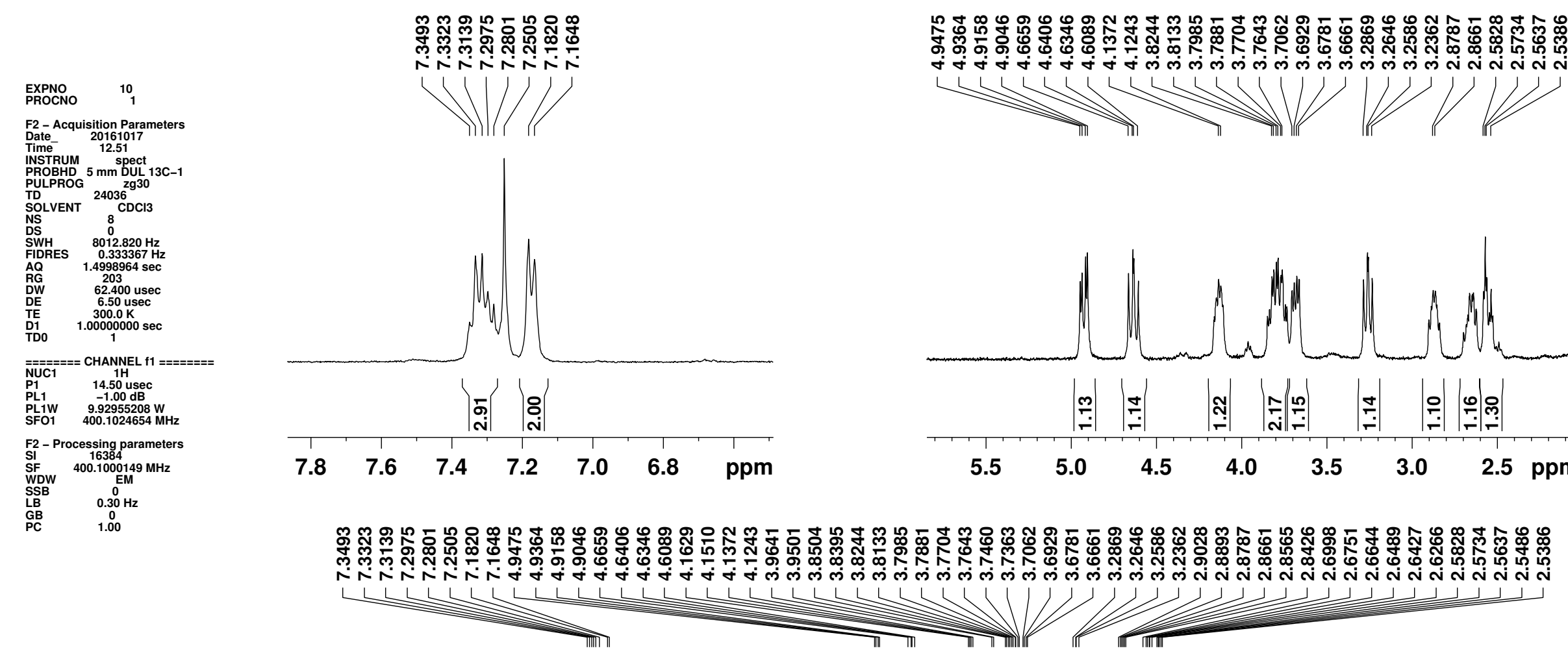

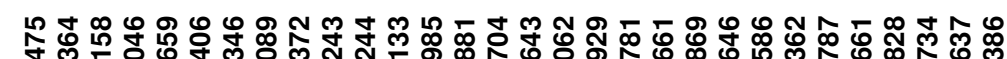

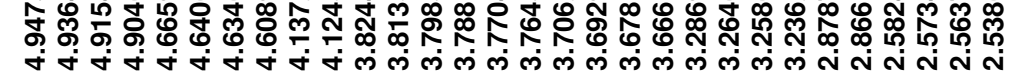

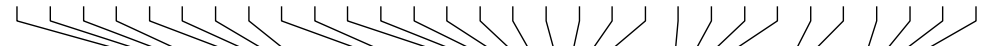

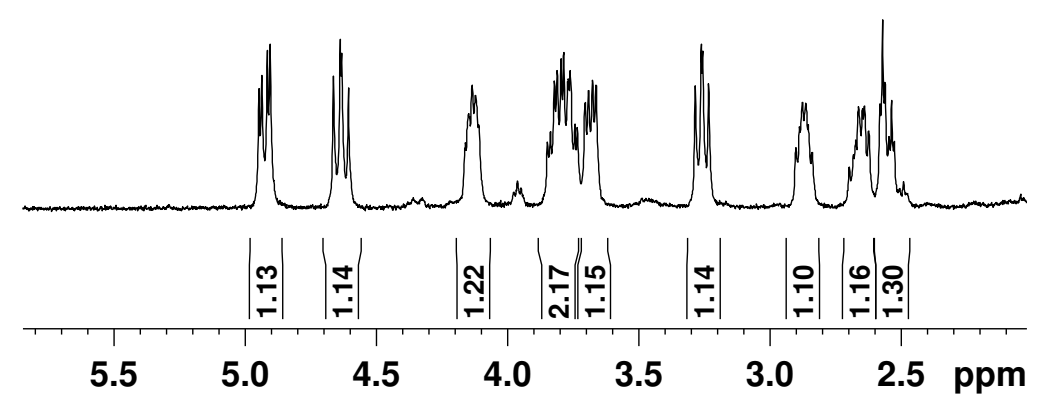<smiles>O=C1CCOC[C@H]1[C@H](C[N+](=O)[O-])c1ccccc1</smiles>

$33 a$ ${ }^{1} \mathrm{H} \mathrm{NMR}, 400 \mathrm{MHz}, \mathrm{CDCl}_{3}$

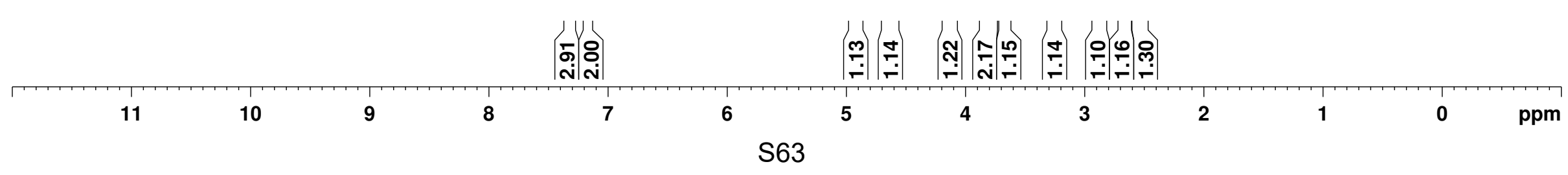




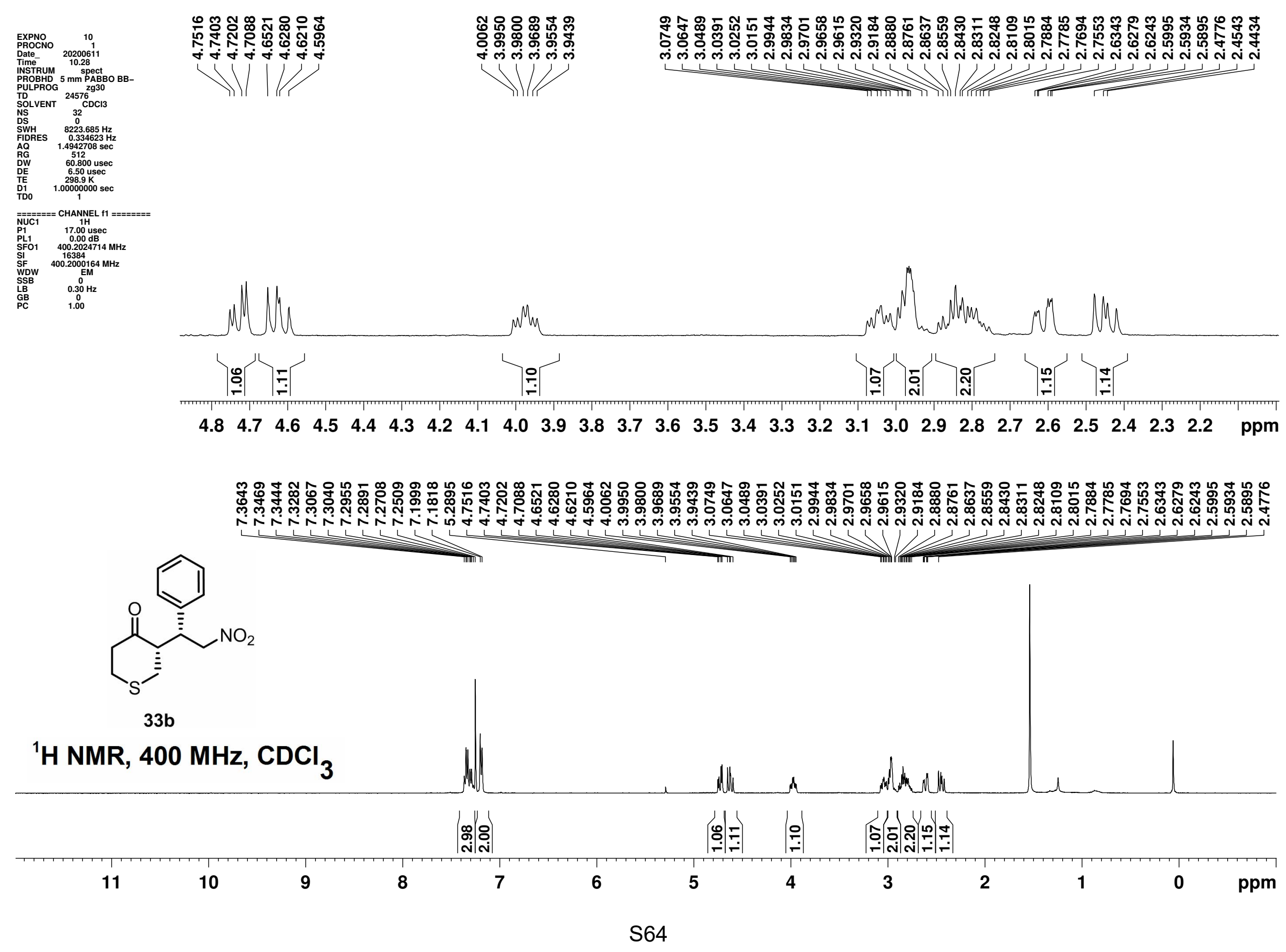



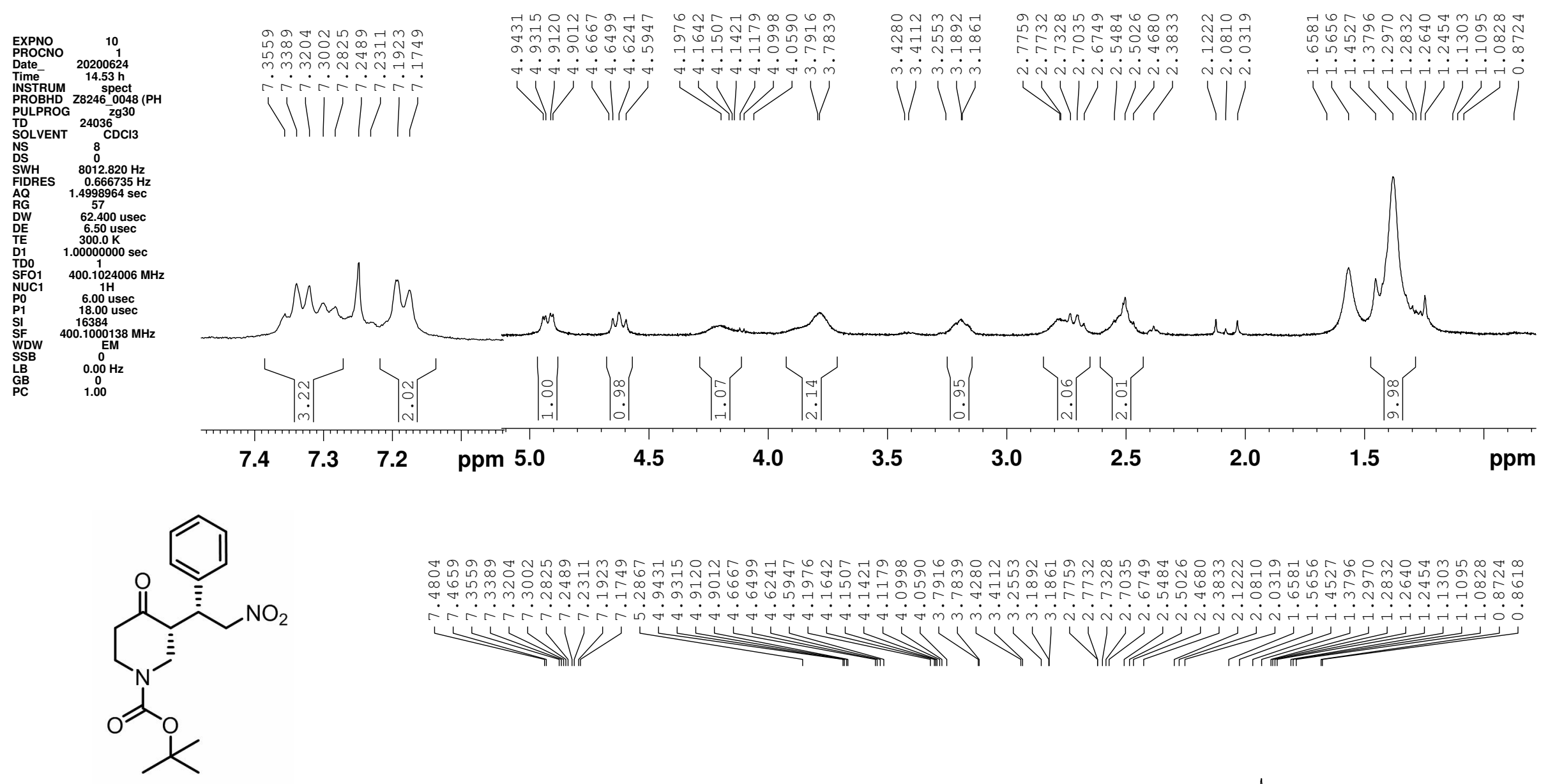

$33 c$

${ }^{1} \mathrm{H}$ NMR, $400 \mathrm{MHz}, \mathrm{CDCl}_{3}$

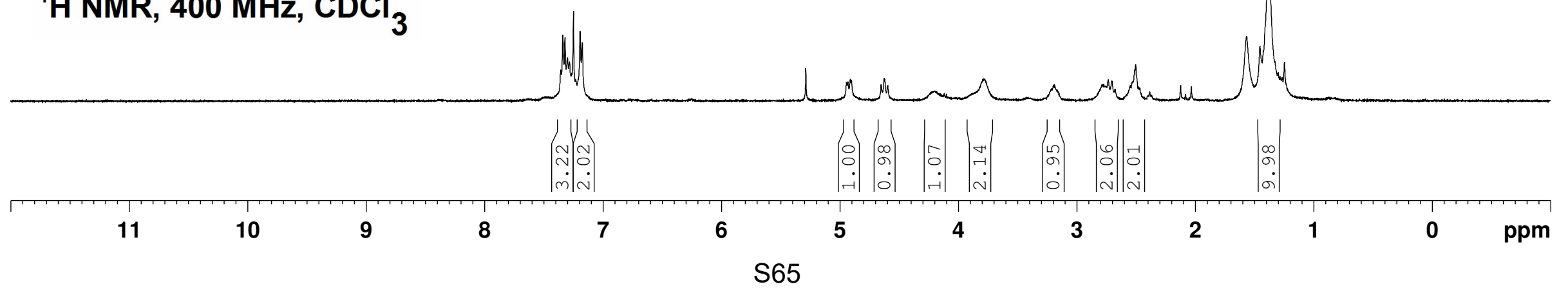



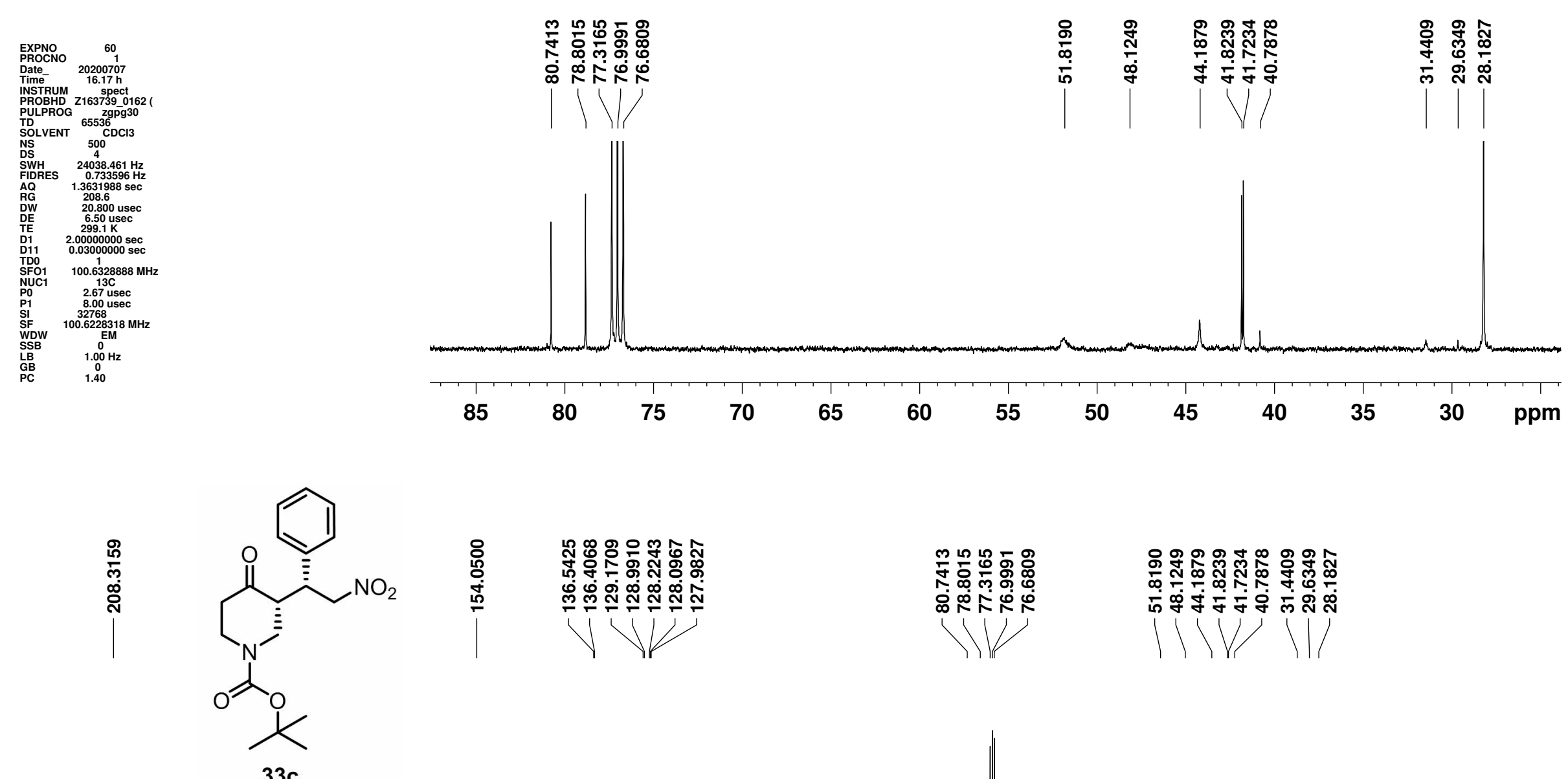

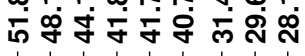

33c

${ }^{13} \mathrm{C}\left\{{ }^{1} \mathrm{H}\right\} \mathrm{NMR}, 100 \mathrm{MHz}, \mathrm{CDCl}_{3}$

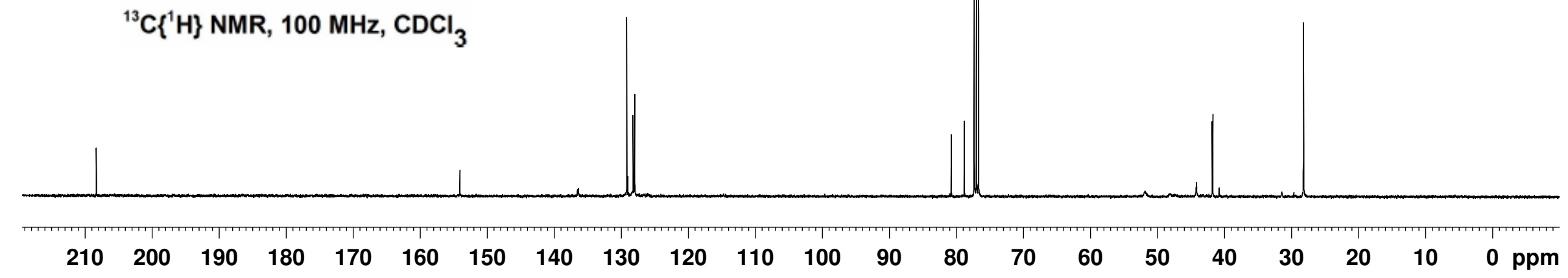




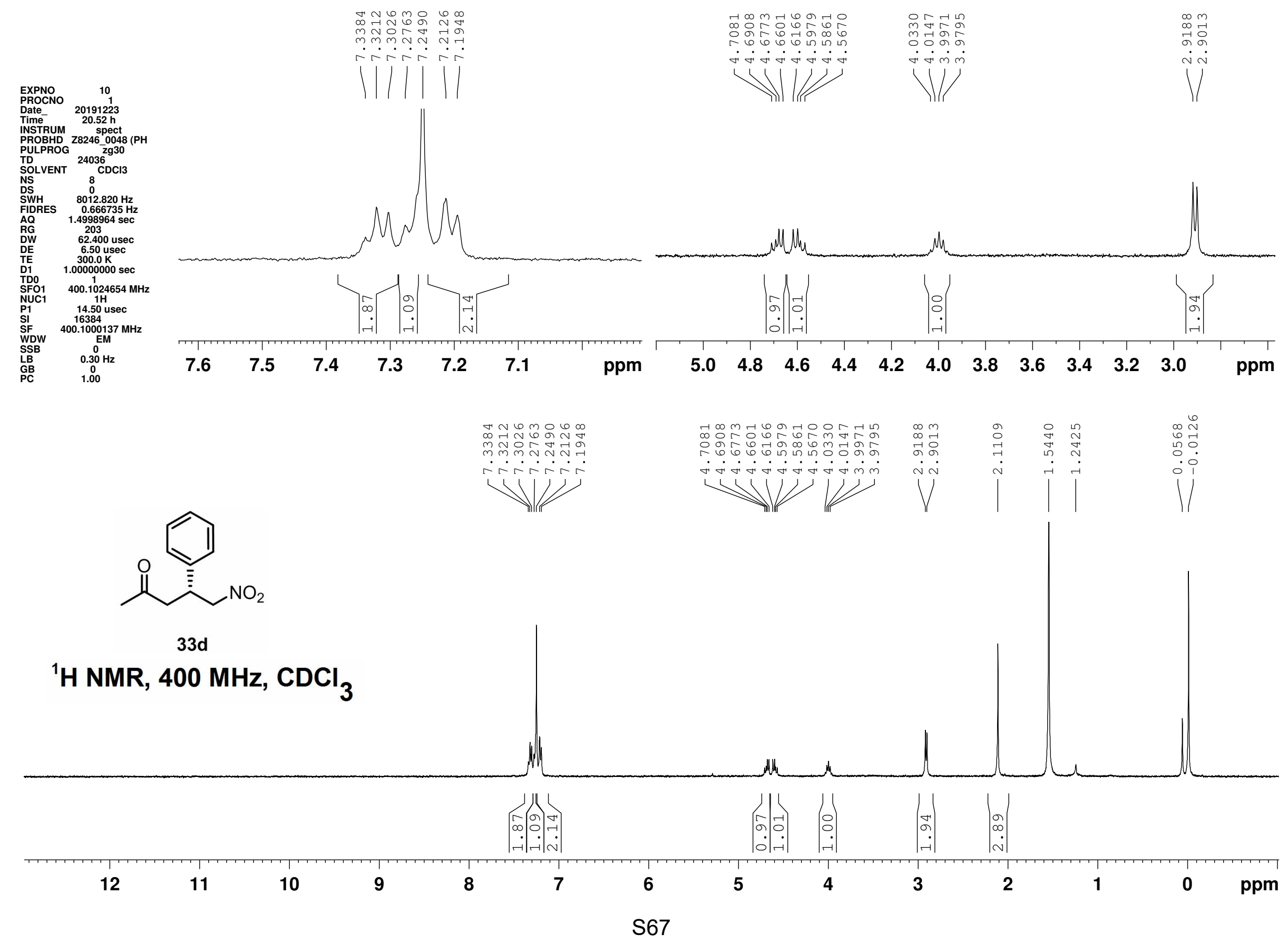



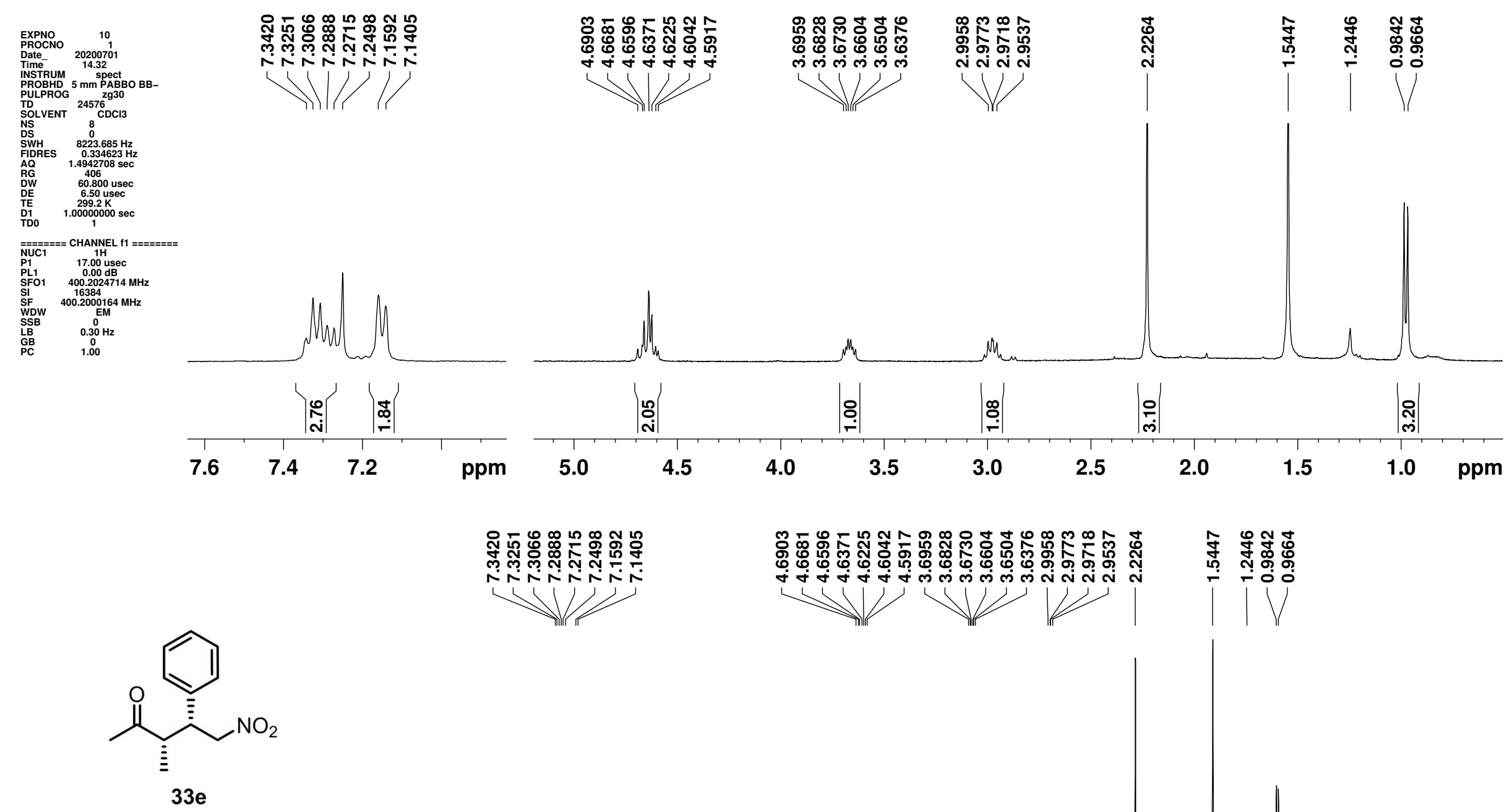

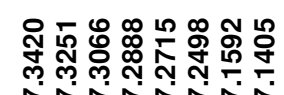

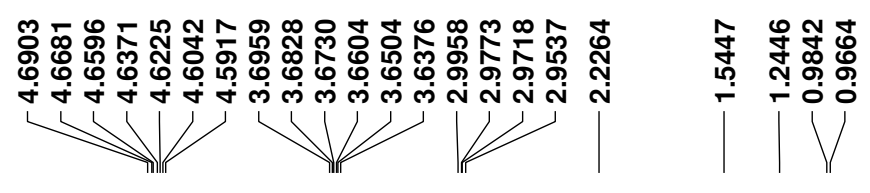

${ }^{1} \mathrm{H}$ NMR, $400 \mathrm{MHz}, \mathrm{CDCl}_{3}$

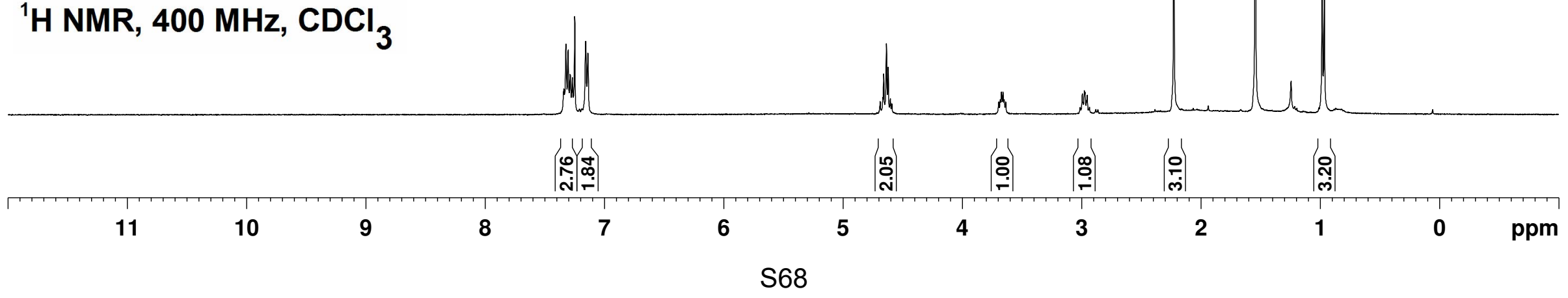



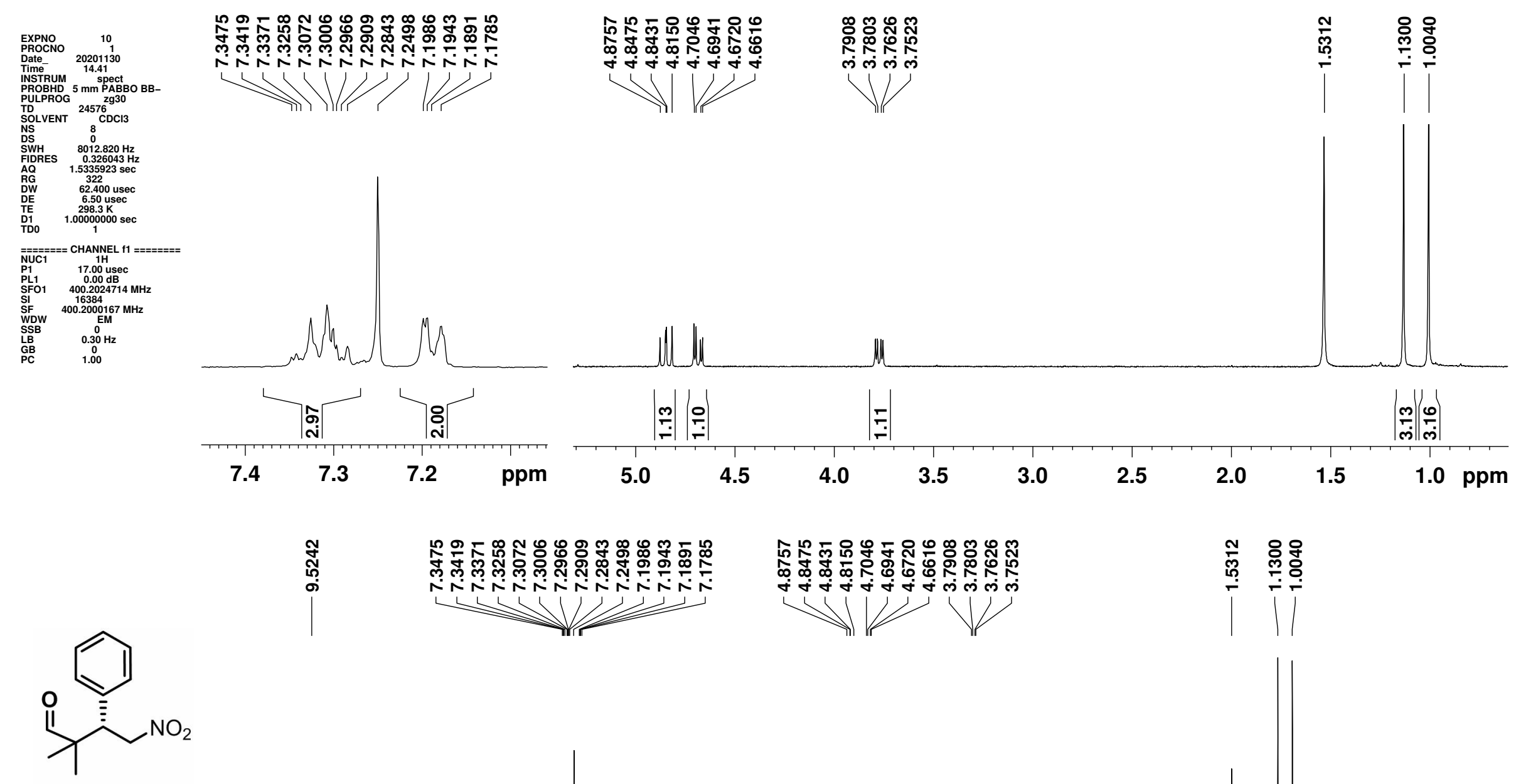

$33 f$

${ }^{1} \mathrm{H}$ NMR, $400 \mathrm{MHz}, \mathrm{CDCl}_{3}$

\begin{tabular}{|c|c|c|c|c|c|c|c|c|c|c|c|}
\hline & : & & & : & & $\left(\begin{array}{l}0 \\
0\end{array}=0\right.$ & $F$ & & & : & \\
\hline 11 & 10 & 9 & 8 & 7 & 6 & 5 & 4 & 3 & 2 & 1 & 0 \\
\hline
\end{tabular}



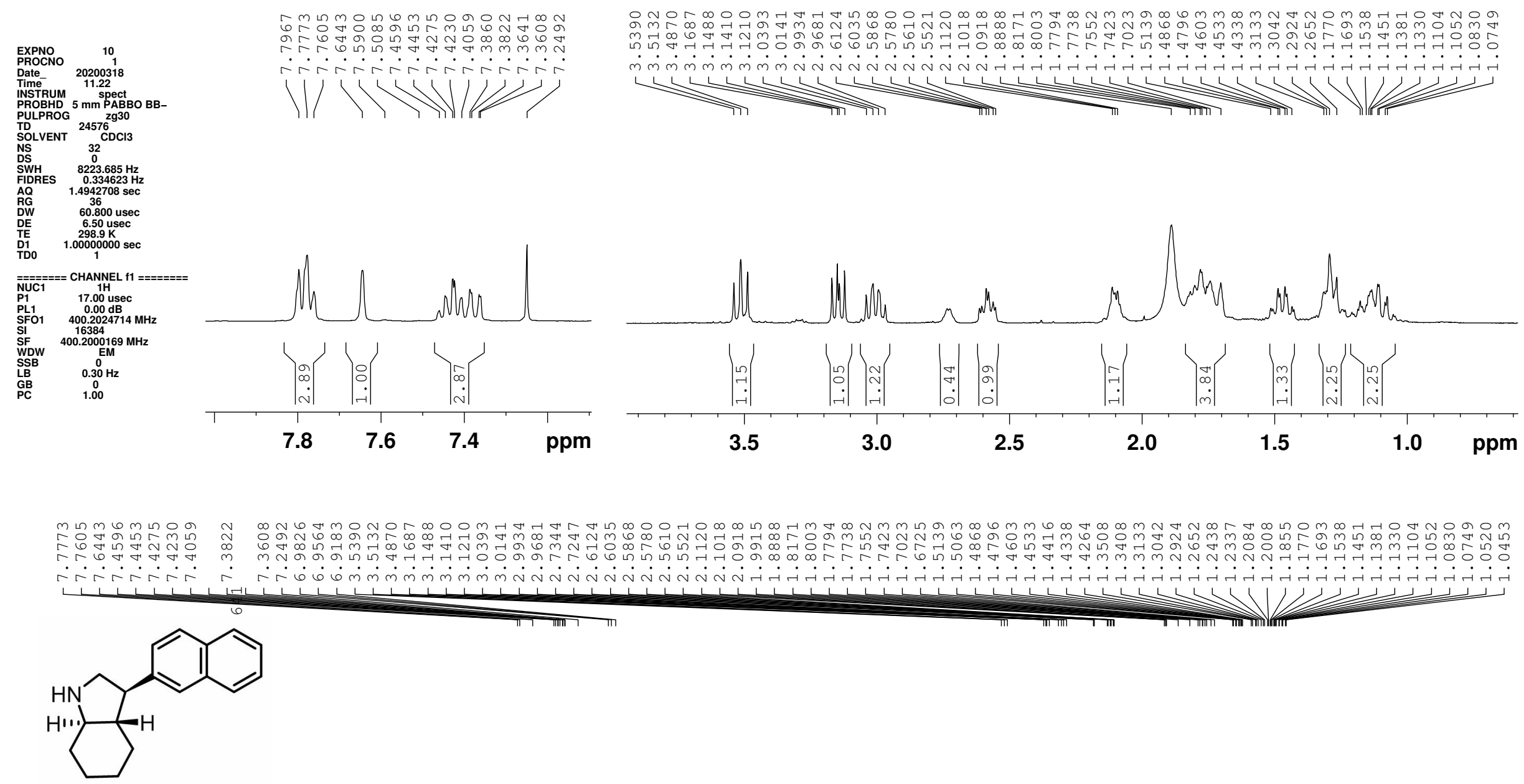

$34 a$

${ }^{1} \mathrm{H}$ NMR, $400 \mathrm{MHz}, \mathrm{CDCl}_{3}$

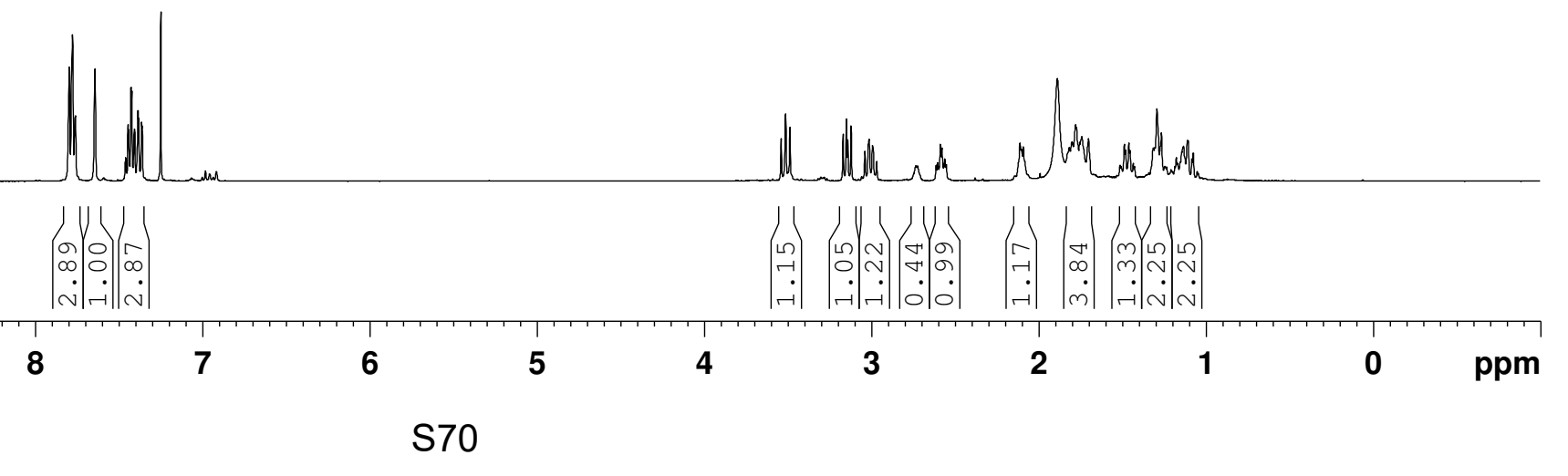



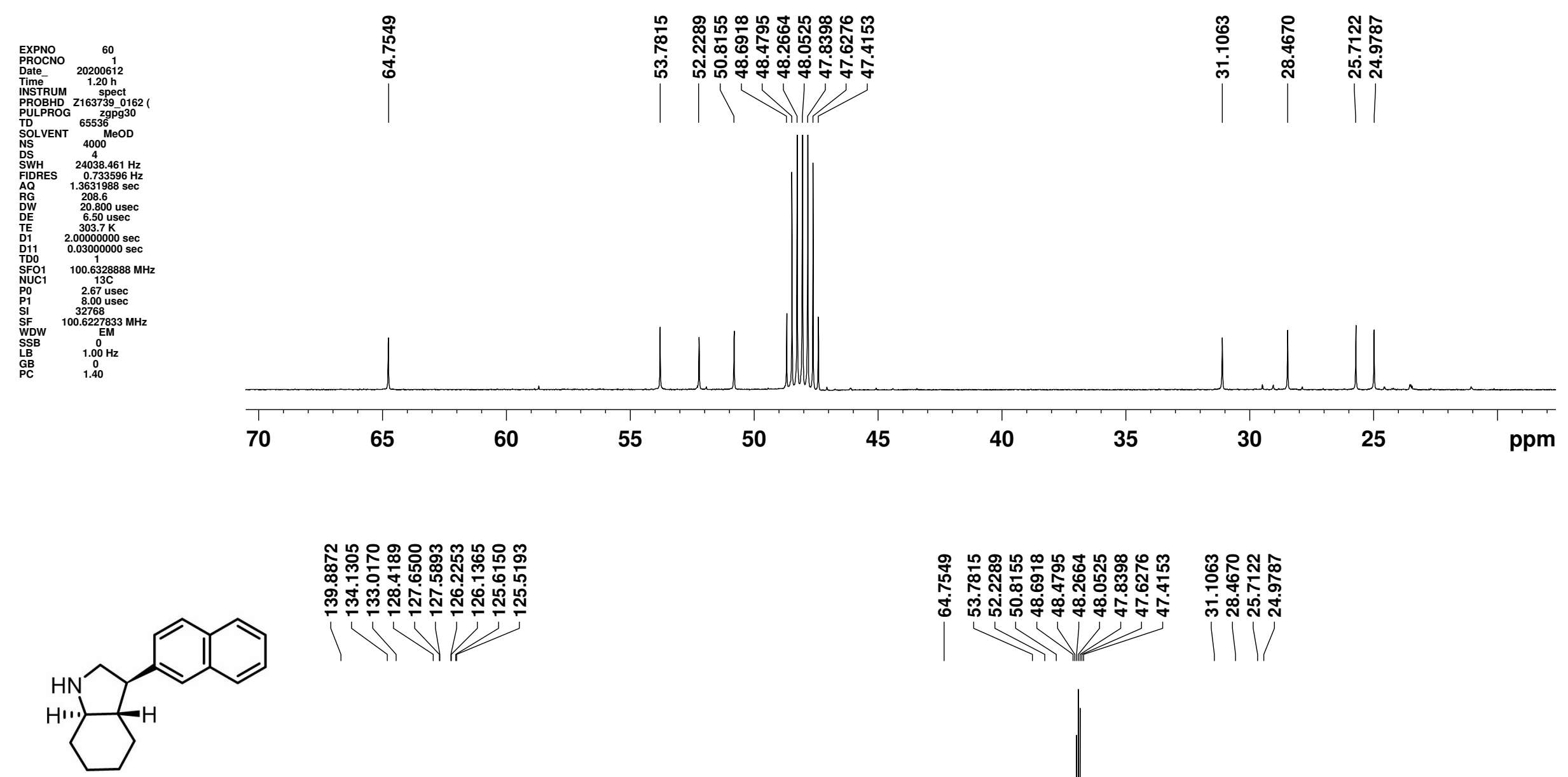

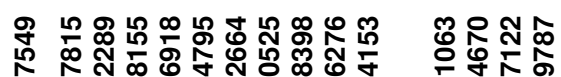

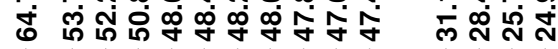

$34 a$

${ }^{13} \mathrm{C}\left\{{ }^{1} \mathrm{H}\right\} \mathrm{NMR}, 100 \mathrm{MHz}, \mathrm{MeOD}$

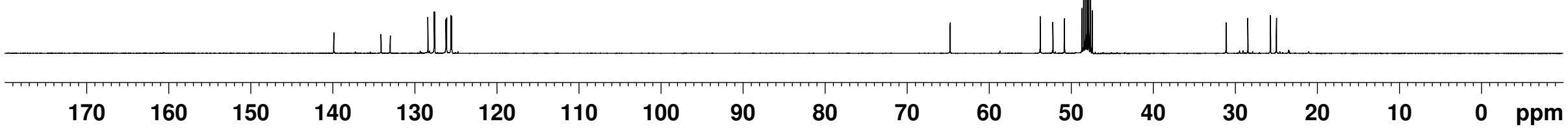



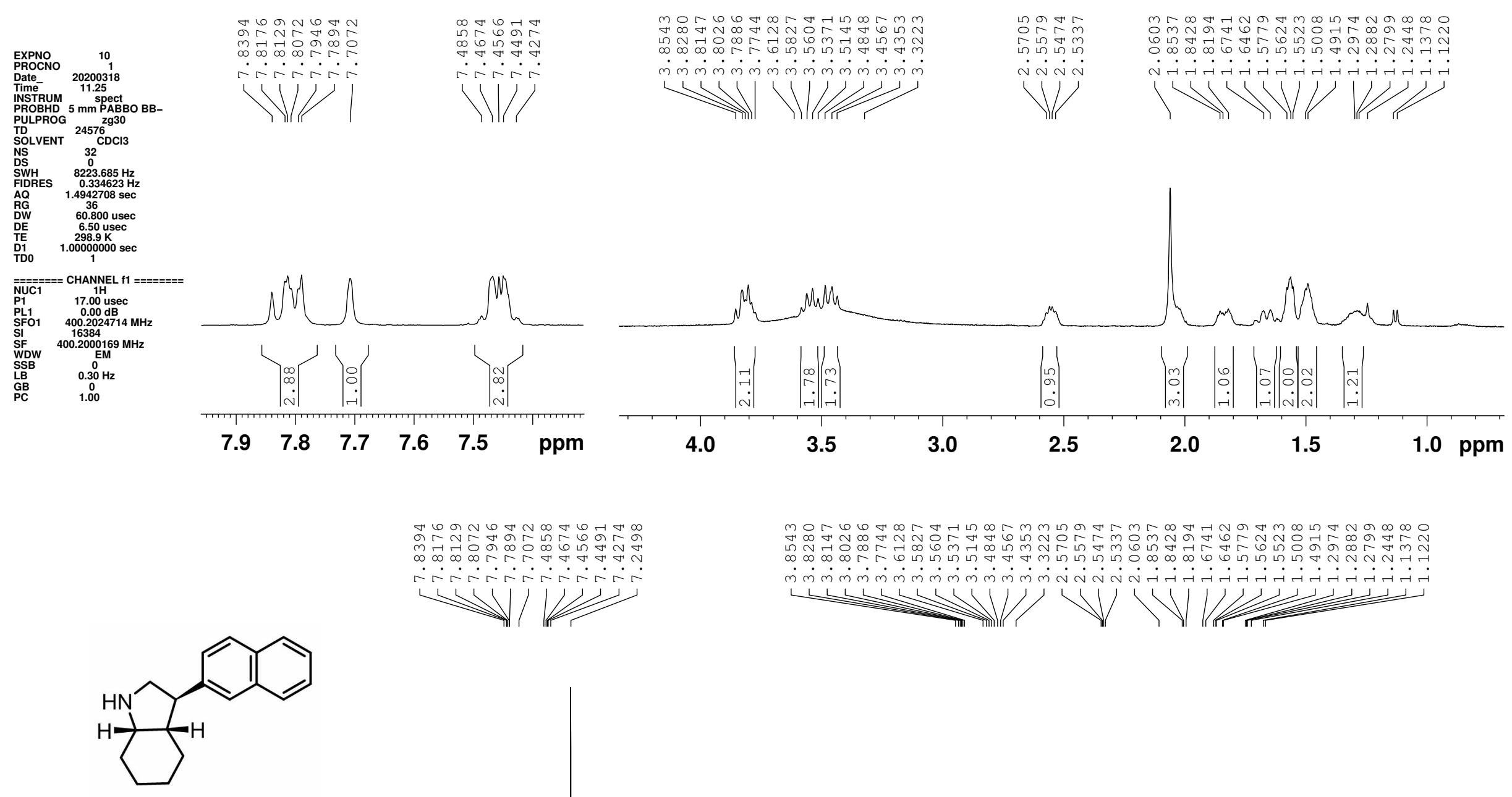

34b

${ }^{1} \mathrm{H} \mathrm{NMR}, 400 \mathrm{MHz}, \mathrm{CDCl}_{3}$

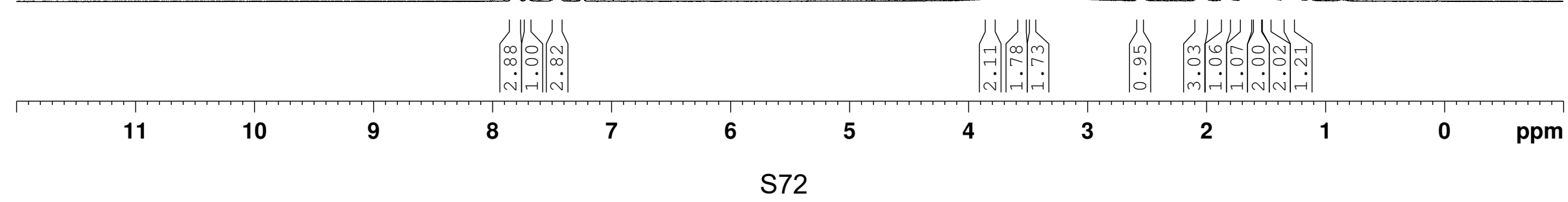



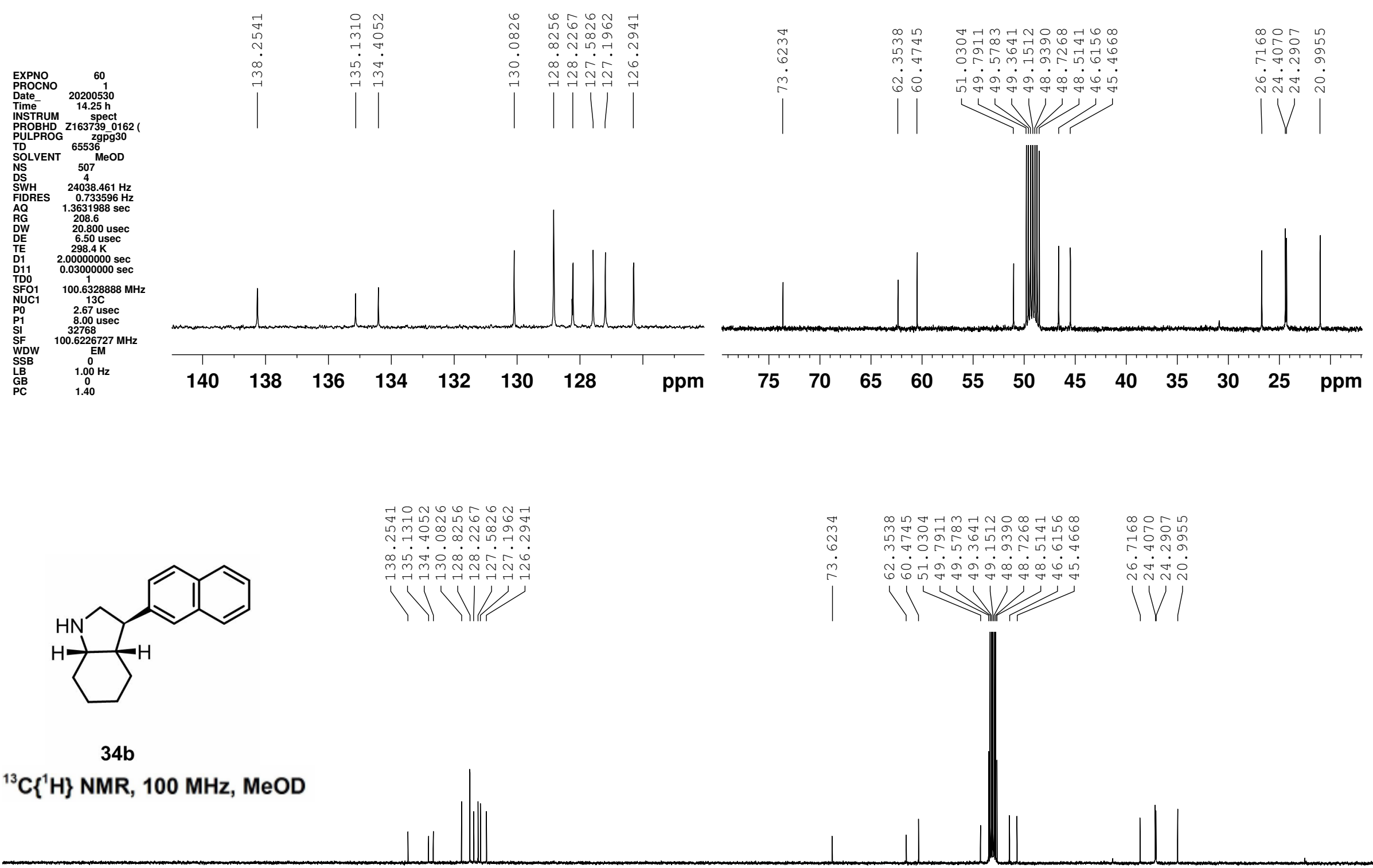

${ }^{13} \mathrm{C}\left\{{ }^{1} \mathrm{H}\right\}$ NMR, $100 \mathrm{MHz}, \mathrm{MeOD}$ 
Column Name : Chiralpak IA (4.6 x $250 \mathrm{~mm}), 5 \mu$

Mobile phase : EtOH : 100

Flow Rate : $0.5 \mathrm{ml} / \mathrm{min}$

Solubility : $\mathrm{MeOH}$
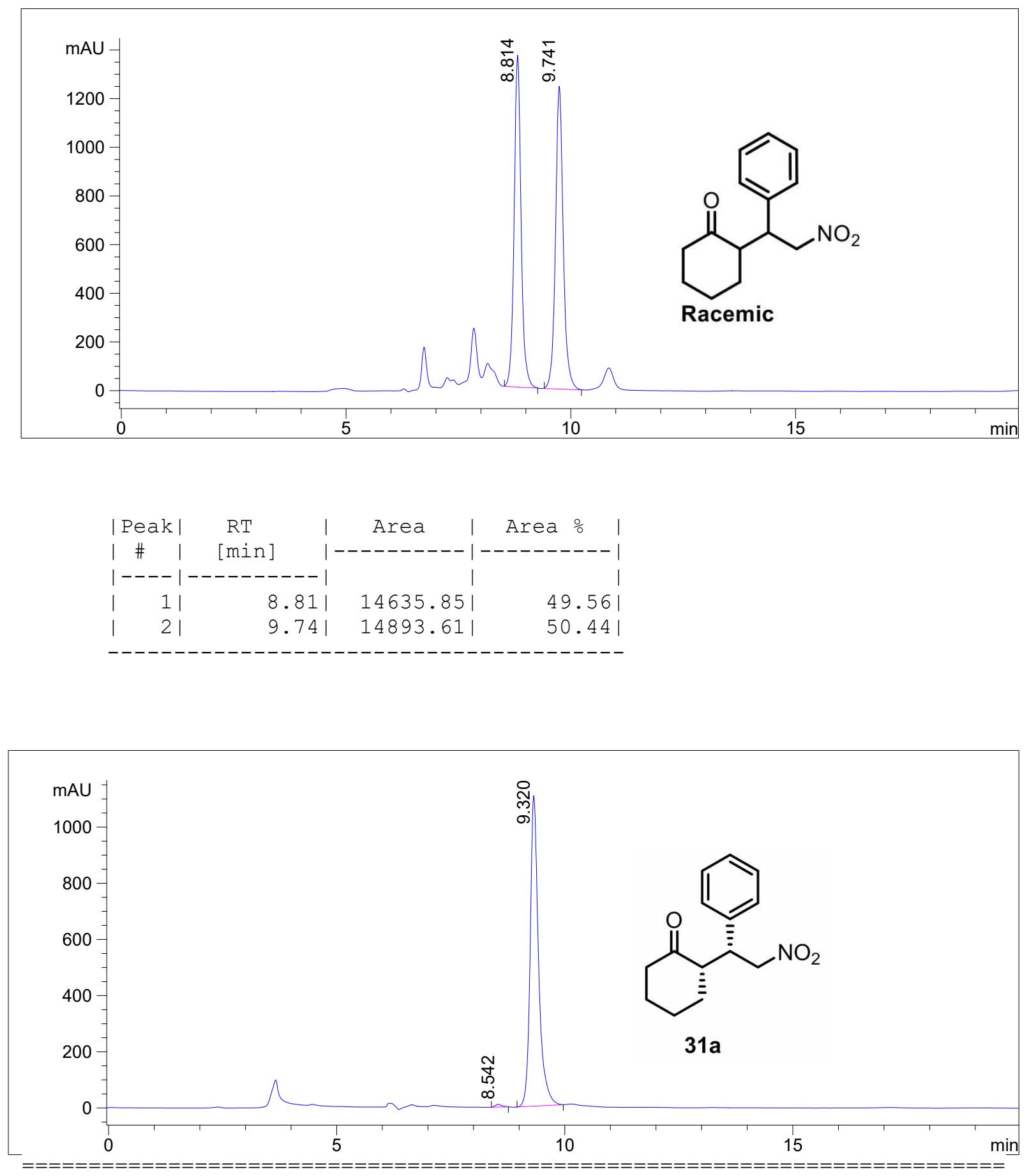

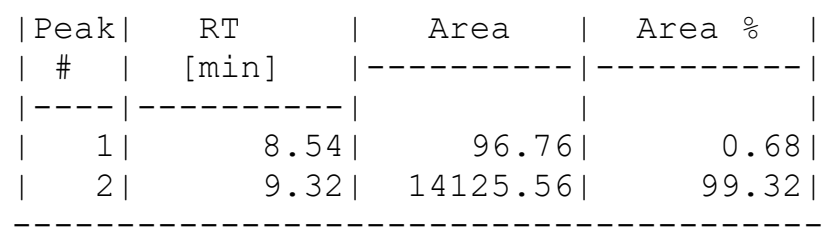


Column Name : CHIRALPAK IA $(4.6 \times 250 \mathrm{~mm}), 5 \mu$

Mobile Phase : HEXANE/ETOH/IPAMINE : 80/20/0.1

Flow Rate : $1.0 \mathrm{ml} / \mathrm{min}$

Solubility : $\mathrm{MeOH}$

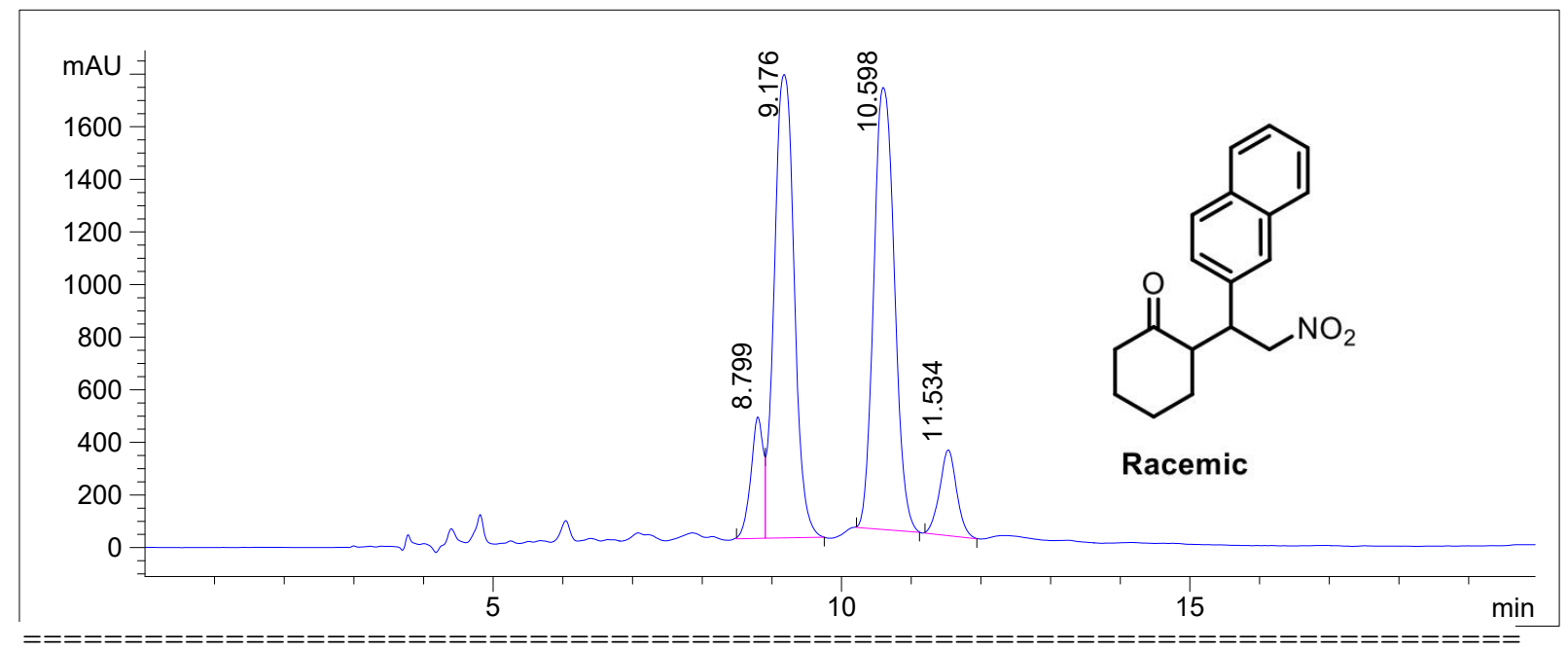

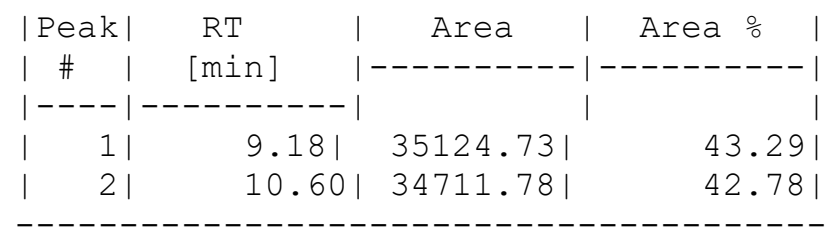

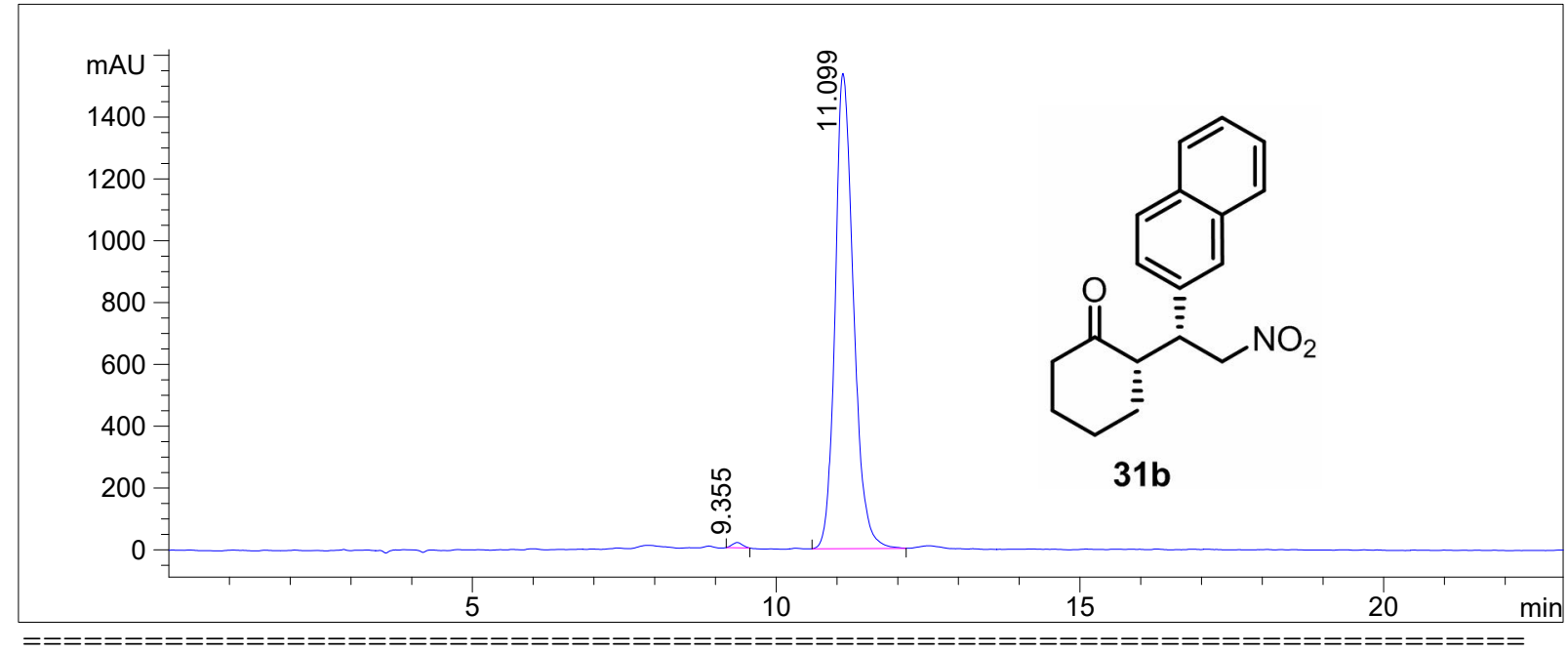

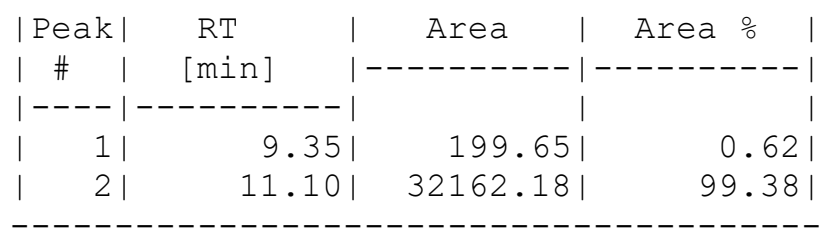


Column Name : Chiralcel OJ-H (4.6 x $250 \mathrm{~mm}), 5 \mu$ Mobile Phase : Hexane/EtoH/IPamine : 80/20/0.1 Flow Rate : $1.0 \mathrm{ml} / \mathrm{min}$

Solubility : $\mathrm{MeOH}$

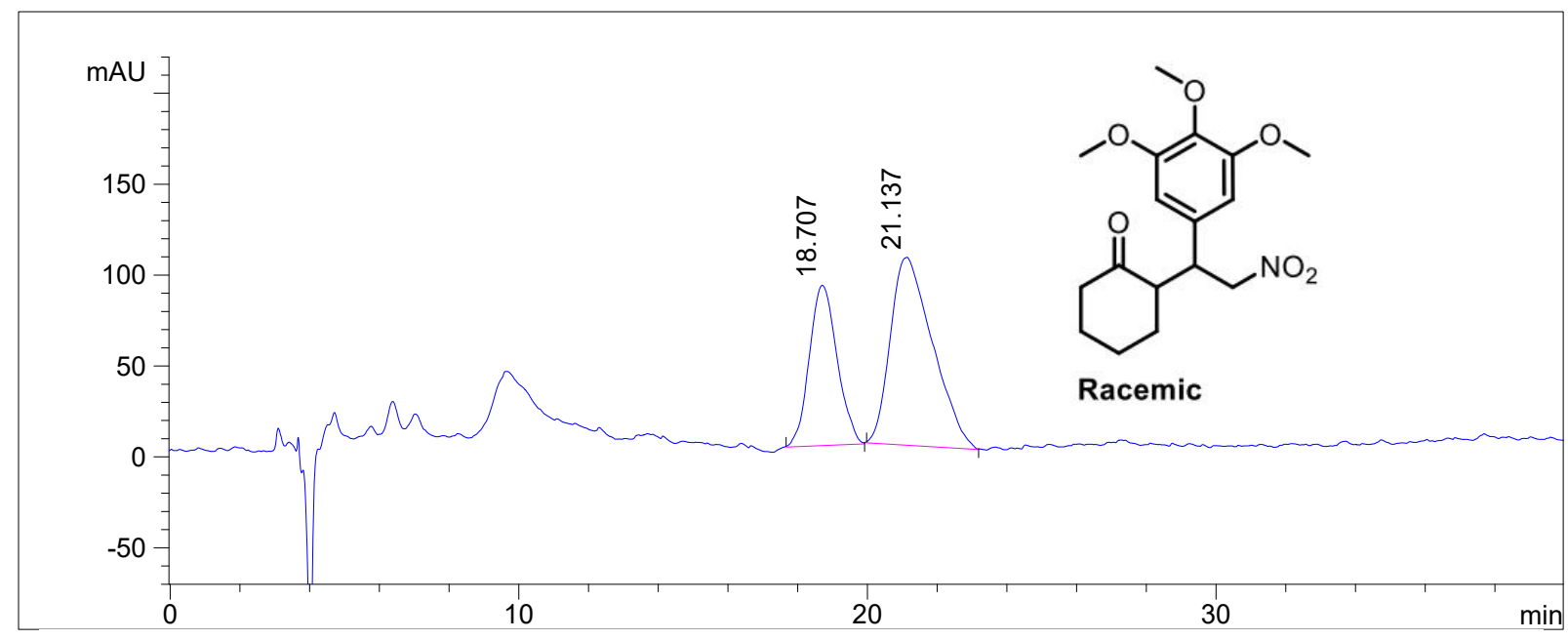

\begin{tabular}{|c|c|c|c|}
\hline Peak| & $\mathrm{RT}$ & Area & $2 a$ \\
\hline | & {$[\mathrm{min}]$} & $-------\mid$ & ---- \\
\hline----1 & $---------\mid$ & | & \\
\hline 11 & $18.71 \mid$ & 4879.761 & 35.94 \\
\hline 21 & $21.14 \mid$ & 8696.661 & 64.06 \\
\hline
\end{tabular}

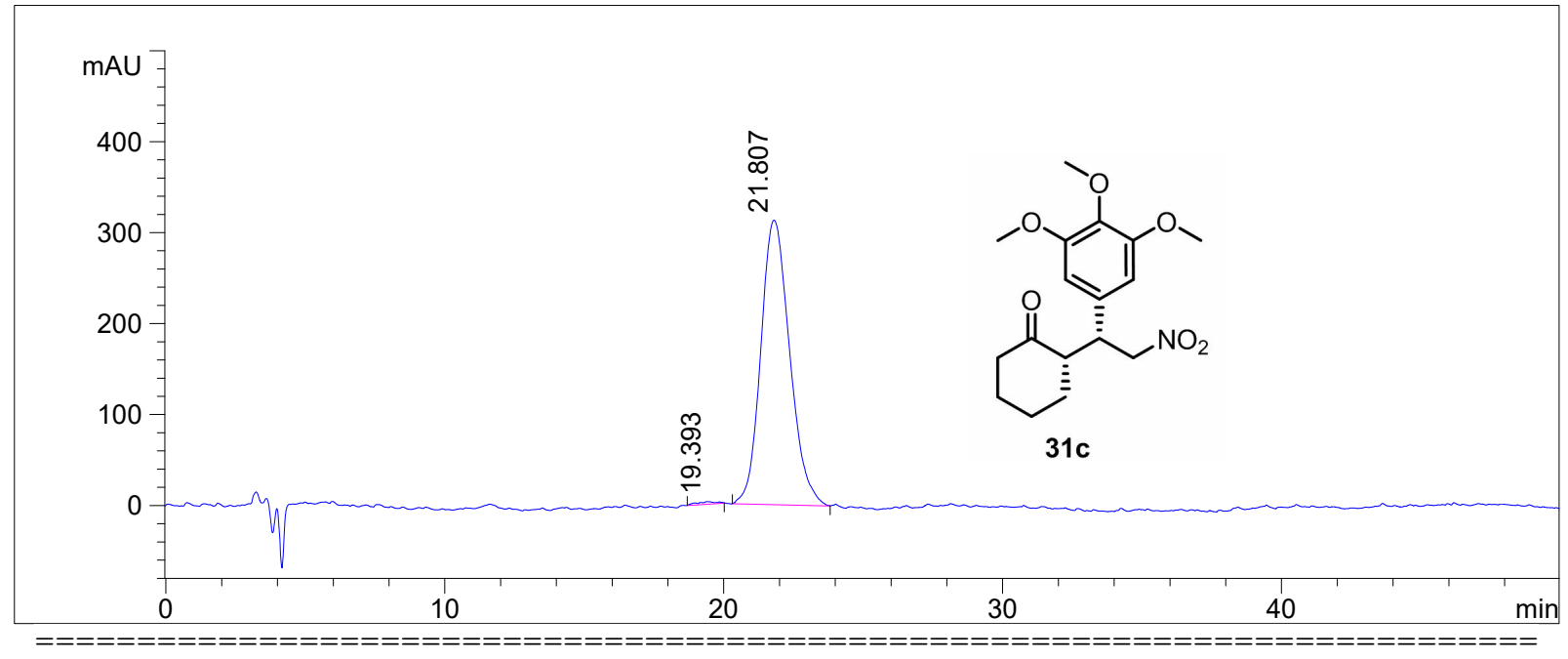

\begin{tabular}{|c|c|c|c|}
\hline & $\mathrm{RT}$ & Area & $a \div$ \\
\hline \multirow{2}{*}{$\begin{array}{c}\mid \text { Peak } \\
\left|\begin{array}{|}\mid \\
\mid \\
|----|\end{array}\right|\end{array}$} & [min $]$ & $----\mid$ & -----1 \\
\hline & $---------\mid$ & | & \\
\hline $1 \mid$ & 19.39 & 142.54 & 0.631 \\
\hline 21 & 21.811 & $22494.87 \mid$ & 99.371 \\
\hline
\end{tabular}


Column Name : Chiralpak IA (4.6 x $250 \mathrm{~mm}), 5 \mu$

Mobile Phase : Hexane/EtoH/IPamine : 80/20/0.1

Flow Rate : $1.0 \mathrm{ml} / \mathrm{min}$

Solubility : $\mathrm{MeOH}$

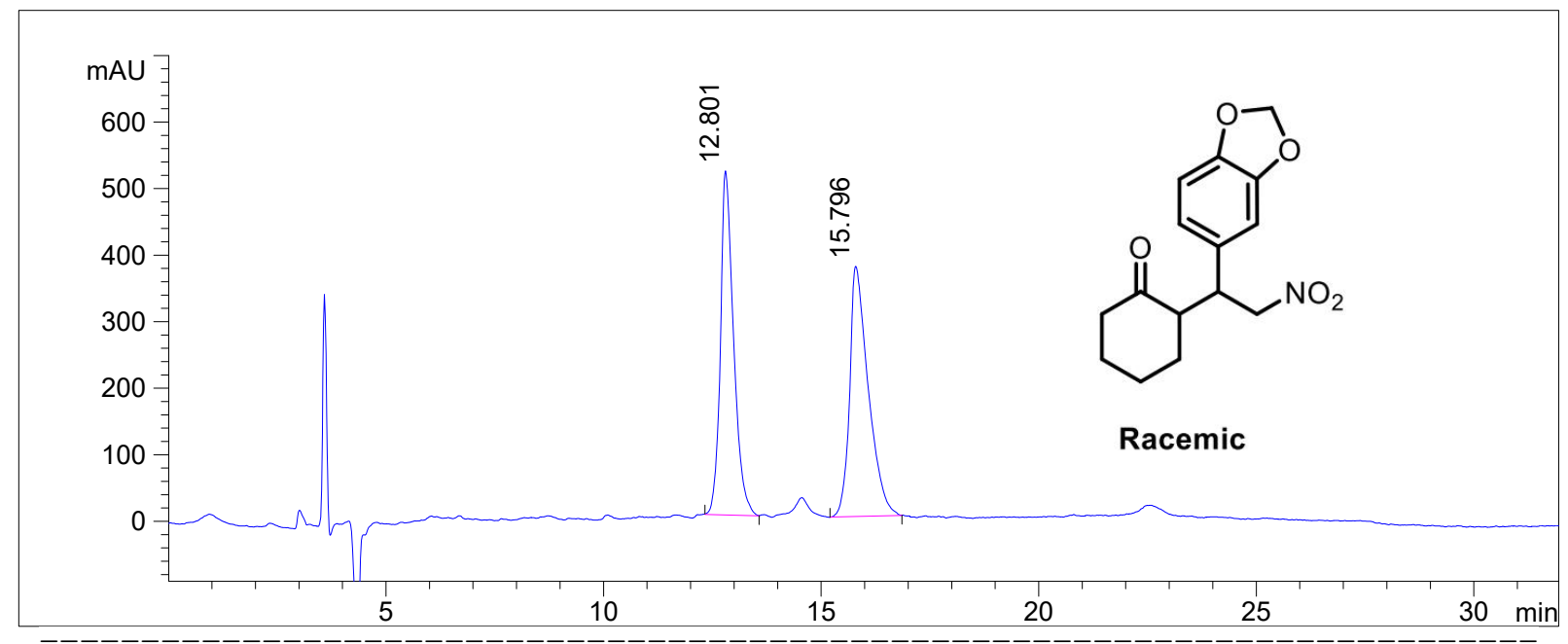

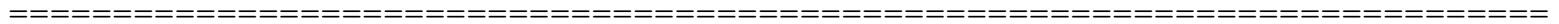

\begin{tabular}{|c|c|c|c|}
\hline | Peak | & RT & Area & ea \\
\hline | \# $\quad$ | & {$[\mathrm{min}]$} & $-----\mid$ & $-----\mid$ \\
\hline$|----|$ & $---------\mid$ & | & \\
\hline | 1 | & 12.80 & $10872.57 \mid$ & 49.29 \\
\hline 21 & $15.80 \mid$ & $11183.94 \mid$ & 50.71 \\
\hline
\end{tabular}

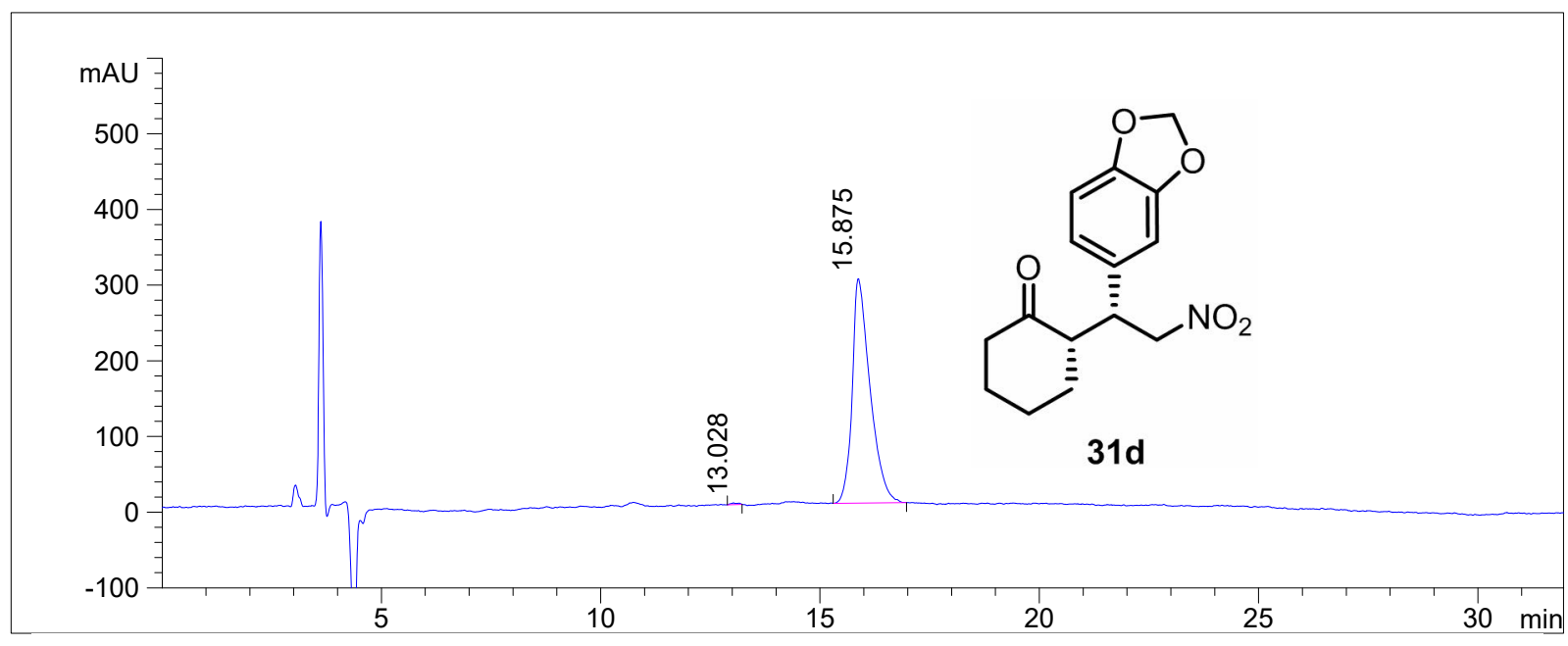

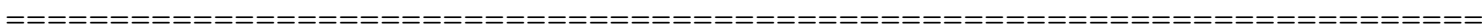

\begin{tabular}{|c|c|c|c|}
\hline Peak| & $\mathrm{RT}$ & Area & Area \% \\
\hline$\# \quad \mid$ & {$[\mathrm{min}]$} & -----1 & ------ \\
\hline----1 & $--------\mid$ & 1 & \\
\hline 1 & 13.031 & 29.671 & \\
\hline 21 & $15.87 \mid$ & 8399.581 & 99 \\
\hline
\end{tabular}


Column Name : Chiralpak IA (4.6 x $250 \mathrm{~mm}), 5 \mu$

Mobile phase : EtOH : 100

Flow Rate : $0.5 \mathrm{ml} / \mathrm{min}$

Solubility : $\mathrm{MeOH}$

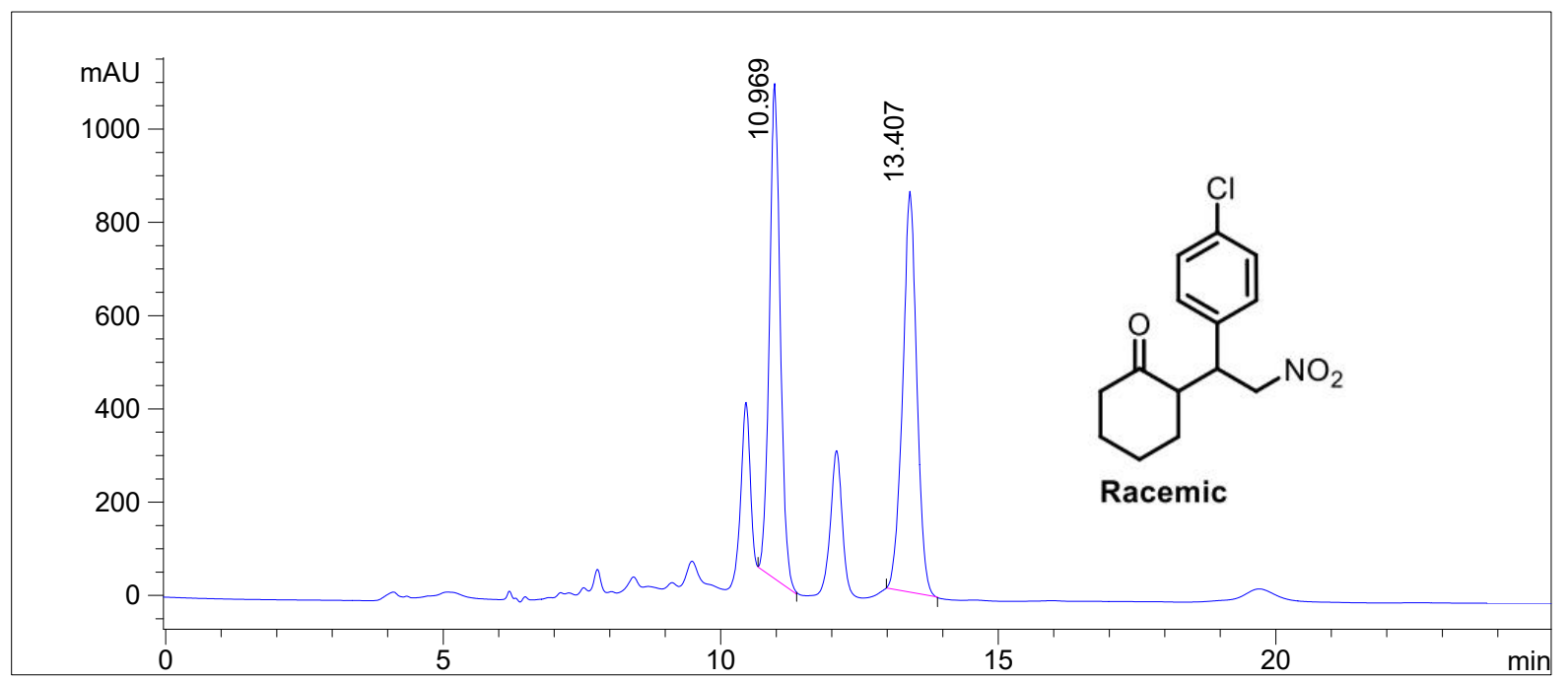

\begin{tabular}{|c|c|c|c|}
\hline | Peak| & $\mathrm{RT}$ & Area & $2 \%$ \\
\hline | $\# \quad$ 1 & [min] & $---------\mid$ & $------\mid$ \\
\hline$|----|$ & $---------\mid$ & | & \\
\hline 11 & $10.97 \mid$ & $14389.37 \mid$ & 48.671 \\
\hline 21 & 13.411 & $15174.06 \mid$ & 51.33 \\
\hline
\end{tabular}

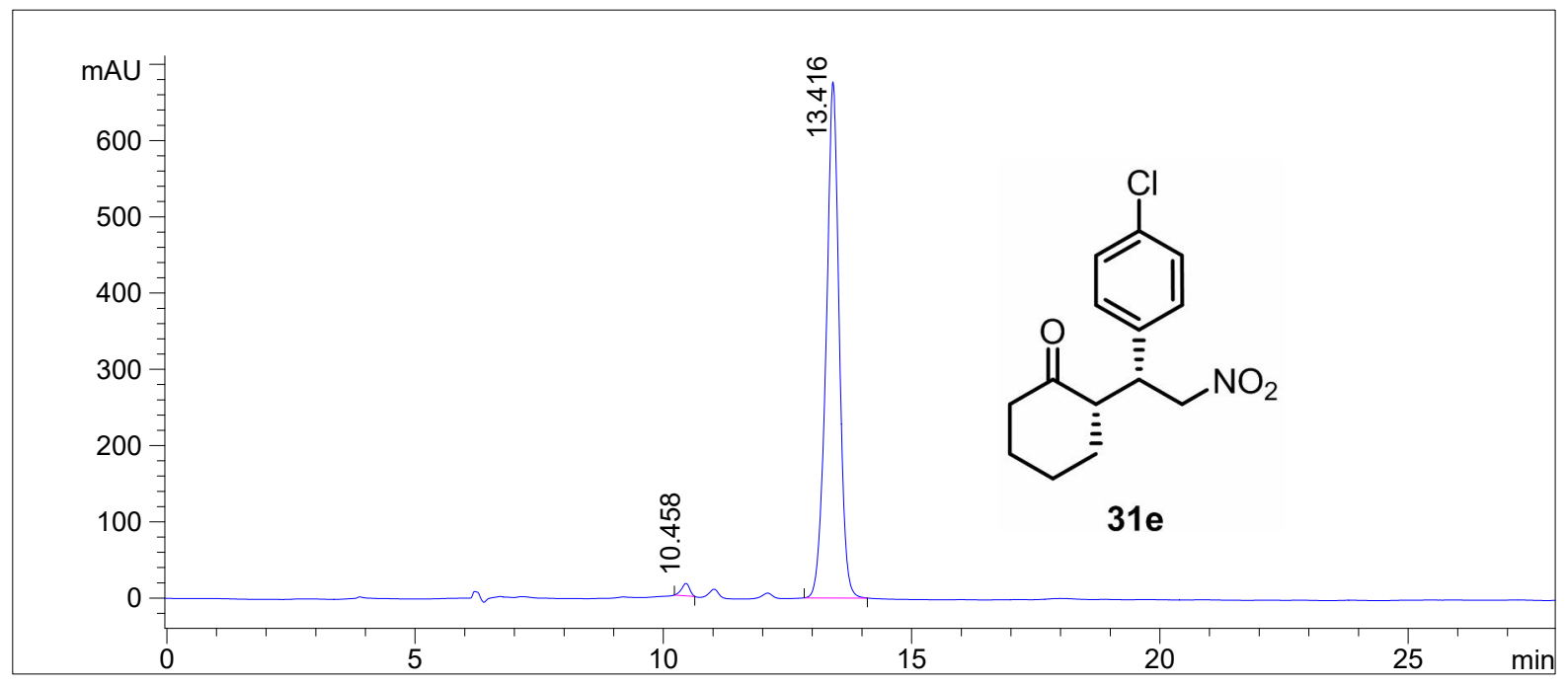

\begin{tabular}{|c|c|c|c|}
\hline | Peak | & $\mathrm{RT}$ & Area & ea $\%$ \\
\hline | \# & [min] & $--------\mid$ & $------\mid$ \\
\hline$|----|$ & $--------\mid$ & | & \\
\hline | 1 | & 10.46 & $187.45 \mid$ & $1.50 \mid$ \\
\hline 21 & $13.42 \mid$ & $12296.12 \mid$ & 98.501 \\
\hline
\end{tabular}


Column Name : Chiralpak IF-3 (4.6 x $250 \mathrm{~mm}), 3 \mu$

Mobile Phase : EtOH : 100

Flow Rate : $0.3 \mathrm{ml} / \mathrm{min}$

Solubility : $\mathrm{MeOH}$
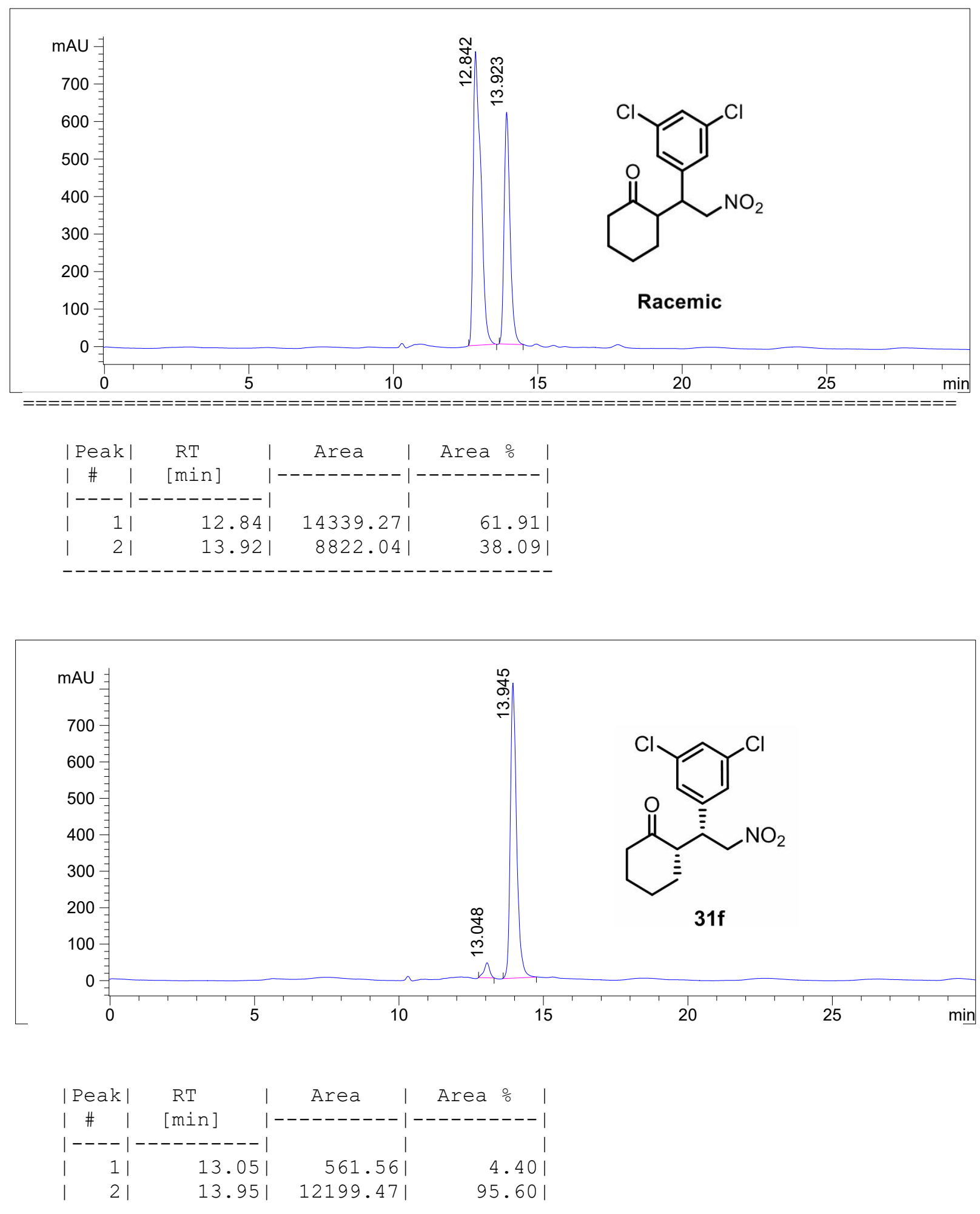
Column Name : Chiralpak IA (4.6 x $250 \mathrm{~mm}), 5 \mu$

Mobile Phase : EtoH : 100

Flow Rate : $0.5 \mathrm{ml} / \mathrm{min}$

Solubility : $\mathrm{MeOH}$

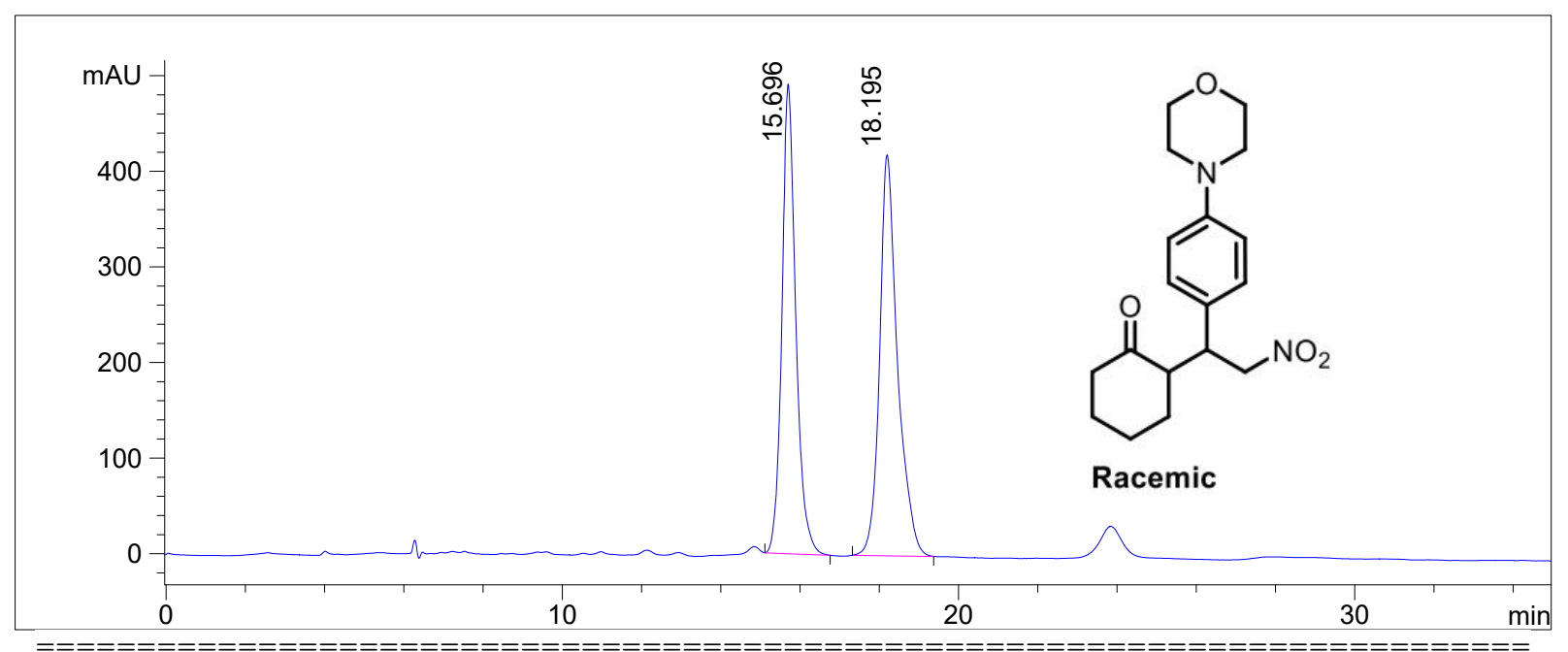

\begin{tabular}{|c|c|c|c|}
\hline | Peak | & $\mathrm{RT}$ & Area & $2 \%$ \\
\hline | \# & [min] & $---------\mid$ & $-----\mid$ \\
\hline$|----|$ & $--------\mid$ & | & \\
\hline | 1 | & 15.70 & 11946.56 & 47.511 \\
\hline 2 & 18.19 & 13197.25 & 52.491 \\
\hline
\end{tabular}

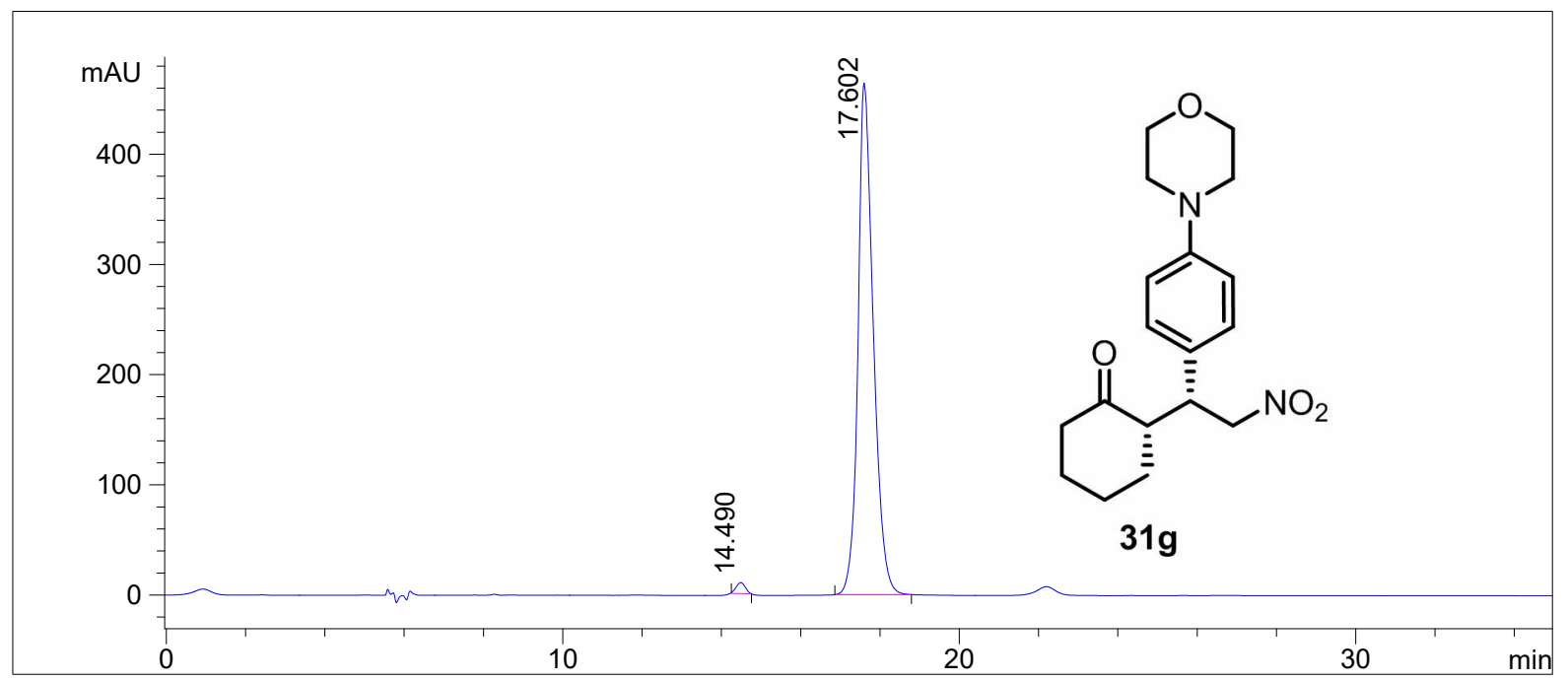

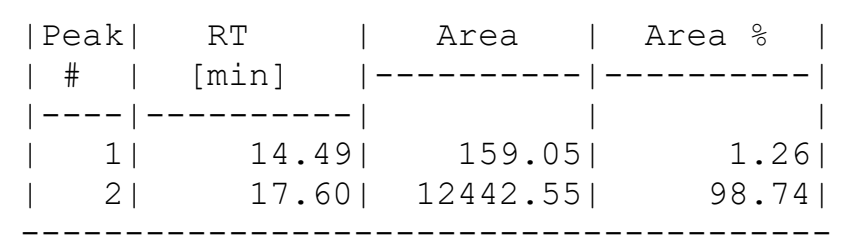


Column Name : Chiralpak IA (4.6 x $250 \mathrm{~mm}), 5 \mu$

Mobile Phase : Hexane/EtoH/IPamine : 80/20/0.1

Flow Rate : $1.0 \mathrm{ml} / \mathrm{min}$

Solubility : $\mathrm{MeOH}$

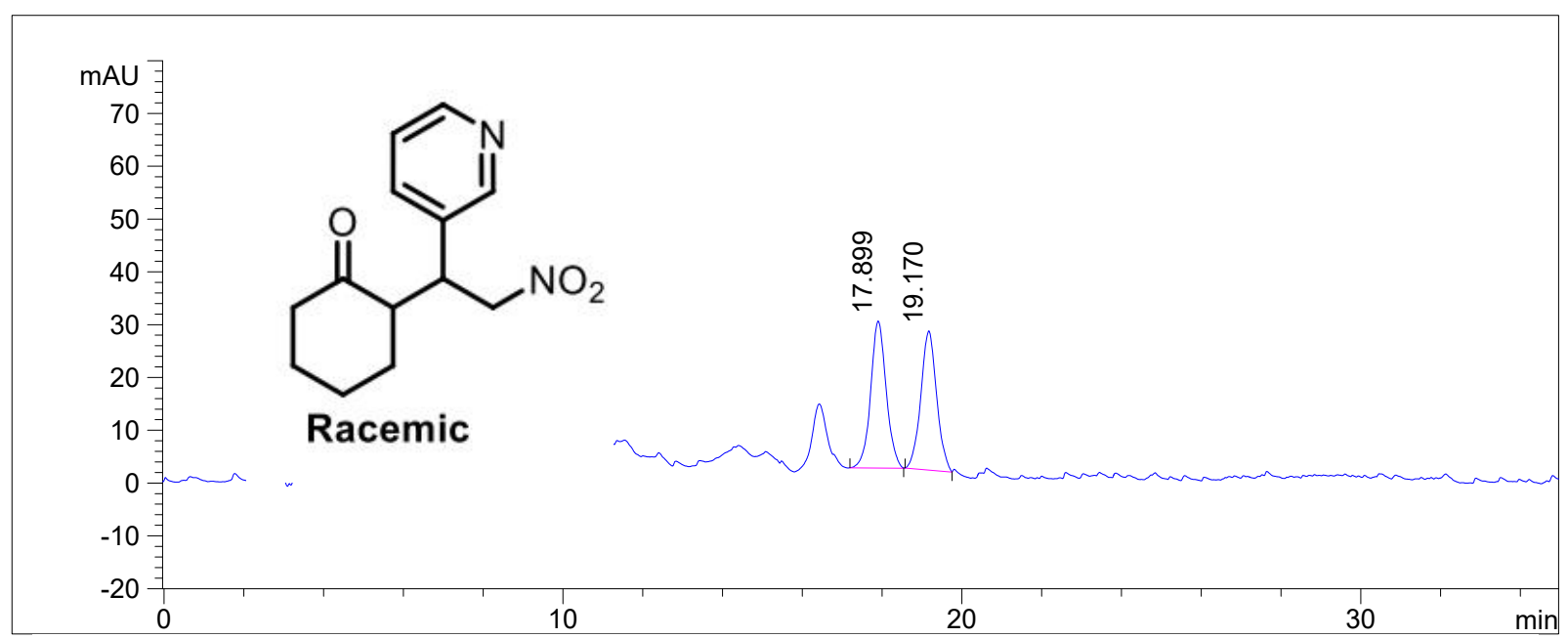

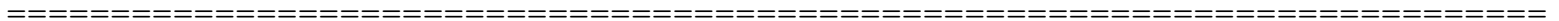

\begin{tabular}{|c|c|c|c|}
\hline | Peak| & RT & Area & ea $\%$ \\
\hline | \# $\quad$ | & [min $]$ & $-------\mid$ & $------\mid$ \\
\hline$|----|$ & $--------\mid$ & | & \\
\hline | 1 | & 17.90 & $753.46 \mid$ & 50.931 \\
\hline 21 & $19.17 \mid$ & $725.84 \mid$ & 49.071 \\
\hline
\end{tabular}

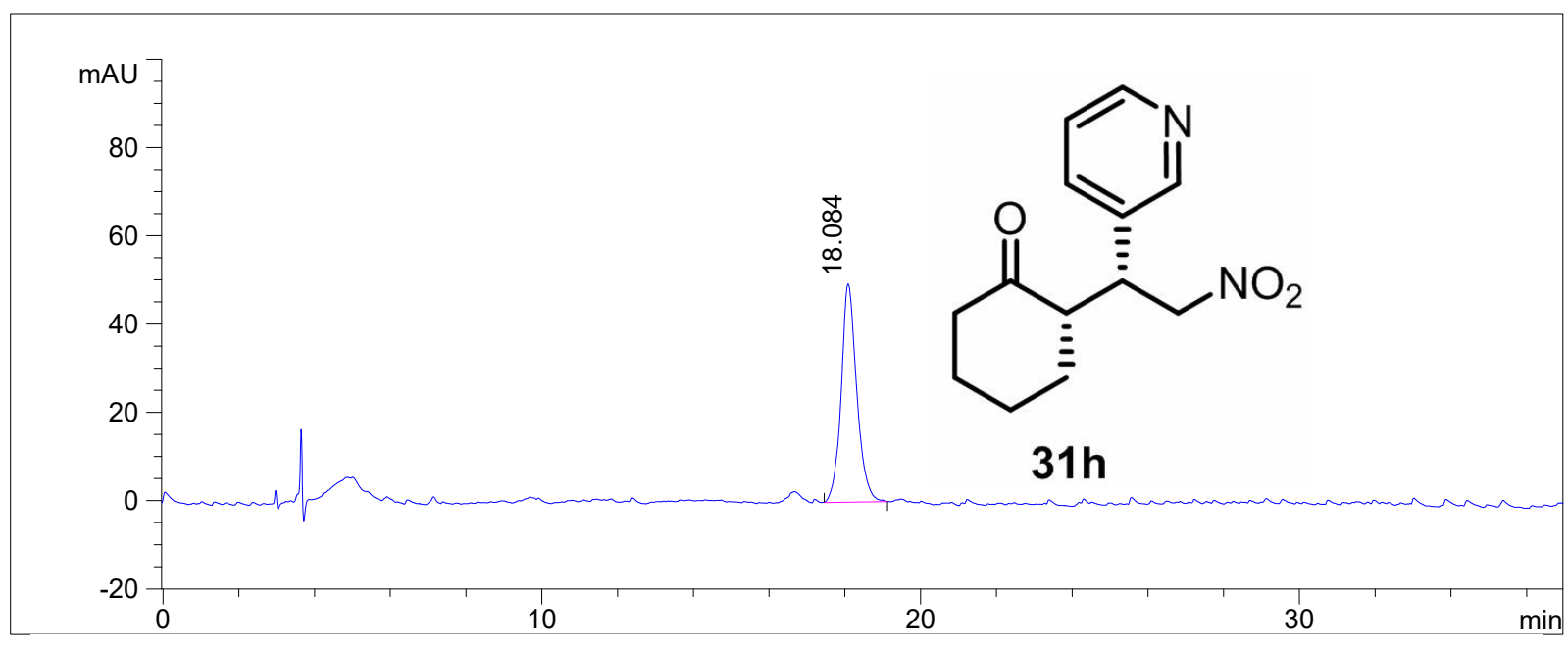

\begin{tabular}{|c|c|c|c|}
\hline | Peak | & RT & Area & rea \\
\hline | \# $\quad$ | & [min] & $--------\mid$ & $------\mid$ \\
\hline$|----|$ & $--------\mid$ & | & \\
\hline | 1 | & 18.08 & $1415.76 \mid$ & $100.00 \mid$ \\
\hline
\end{tabular}


Column Name : Chiralpak IA (4.6 x $250 \mathrm{~mm}), 5 \mu$

Mobile Phase : Hexane/EtoH/IPamine : 80/20/0.1

Flow Rate : $1.0 \mathrm{ml} / \mathrm{min}$

Solubility : $\mathrm{MeOH}$

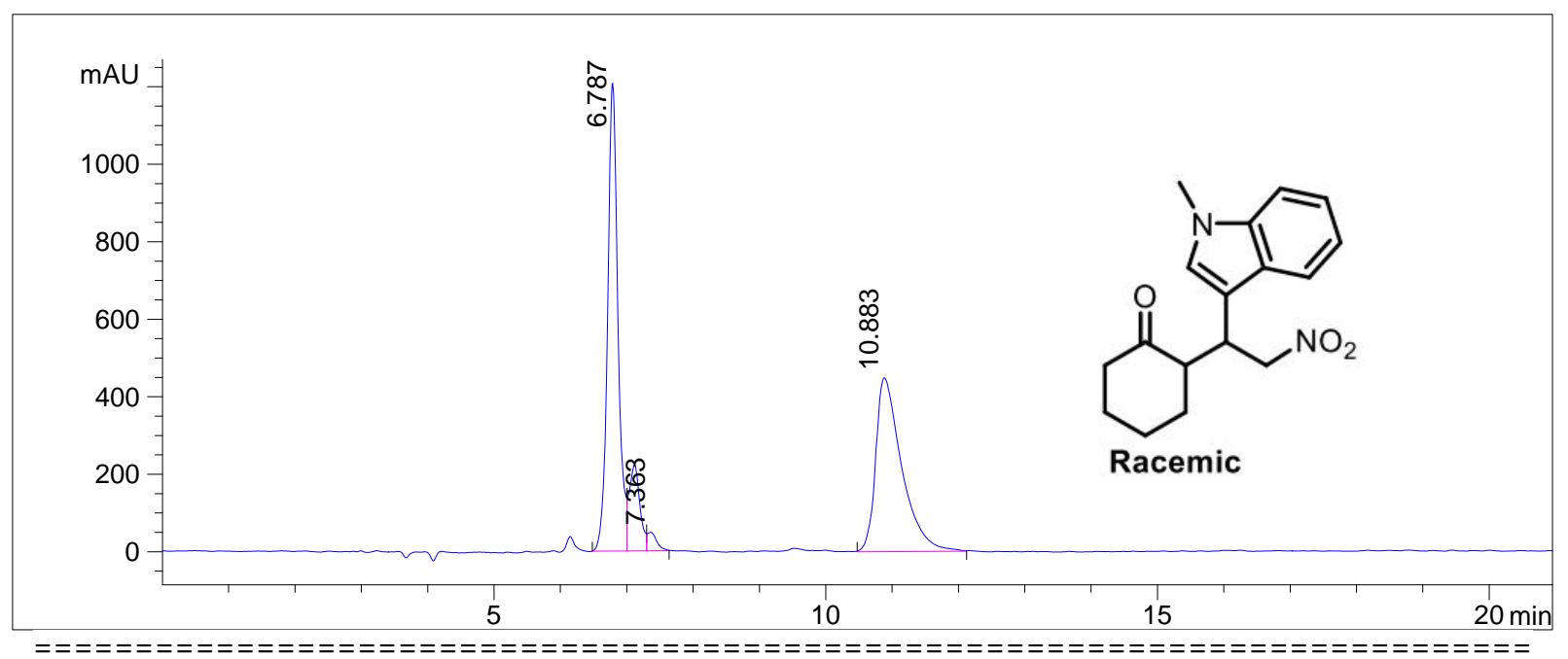

\begin{tabular}{|c|c|c|c|}
\hline | Peak | & RT & Area & rea $\%$ \\
\hline | \# 1 & $\min ] \quad \mid$ & $----------\mid$ & $-------\mid$ \\
\hline$|----|$ & $--------\mid$ & | & \\
\hline $1 \quad 1 \mid$ & 6.791 & 13060.47 & 46.321 \\
\hline $2 \mid$ & 10.88 & 12183.00 & 43.211 \\
\hline
\end{tabular}

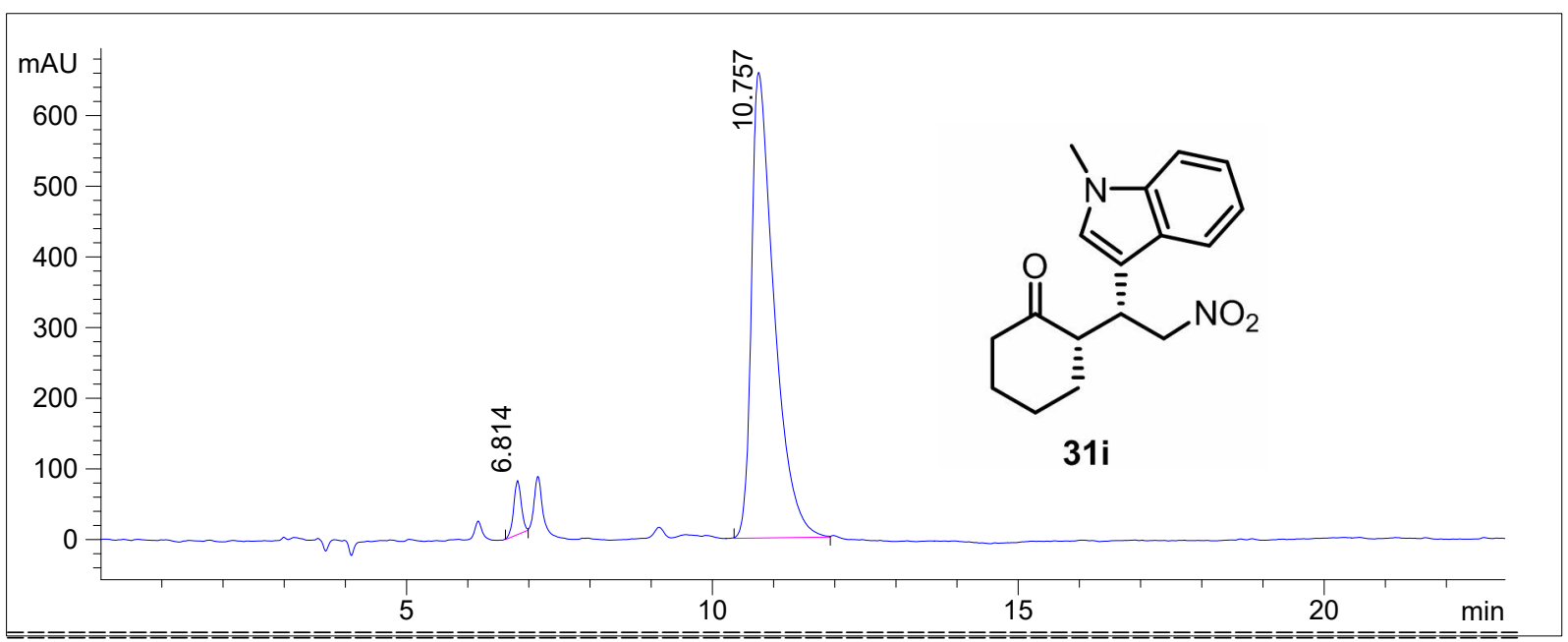

\begin{tabular}{|c|c|c|c|}
\hline | Peak | & $\mathrm{RT}$ & Area & $a$ \\
\hline | \# & [min] & $--------\mid$ & $-----\mid$ \\
\hline$|----|$ & $---------\mid$ & | & \\
\hline | 1 | & 6.81 & 659.211 & 3.741 \\
\hline 2 & $10.76 \mid$ & $16962.65 \mid$ & 96.261 \\
\hline
\end{tabular}


Column Name : Chiralpak IA (4.6 x 250 mm), $5 \mu$

Mobile Phase : EtOH : 100

Flow Rate : $0.5 \mathrm{ml} / \mathrm{min}$

Solubility : $\mathrm{MeOH}$
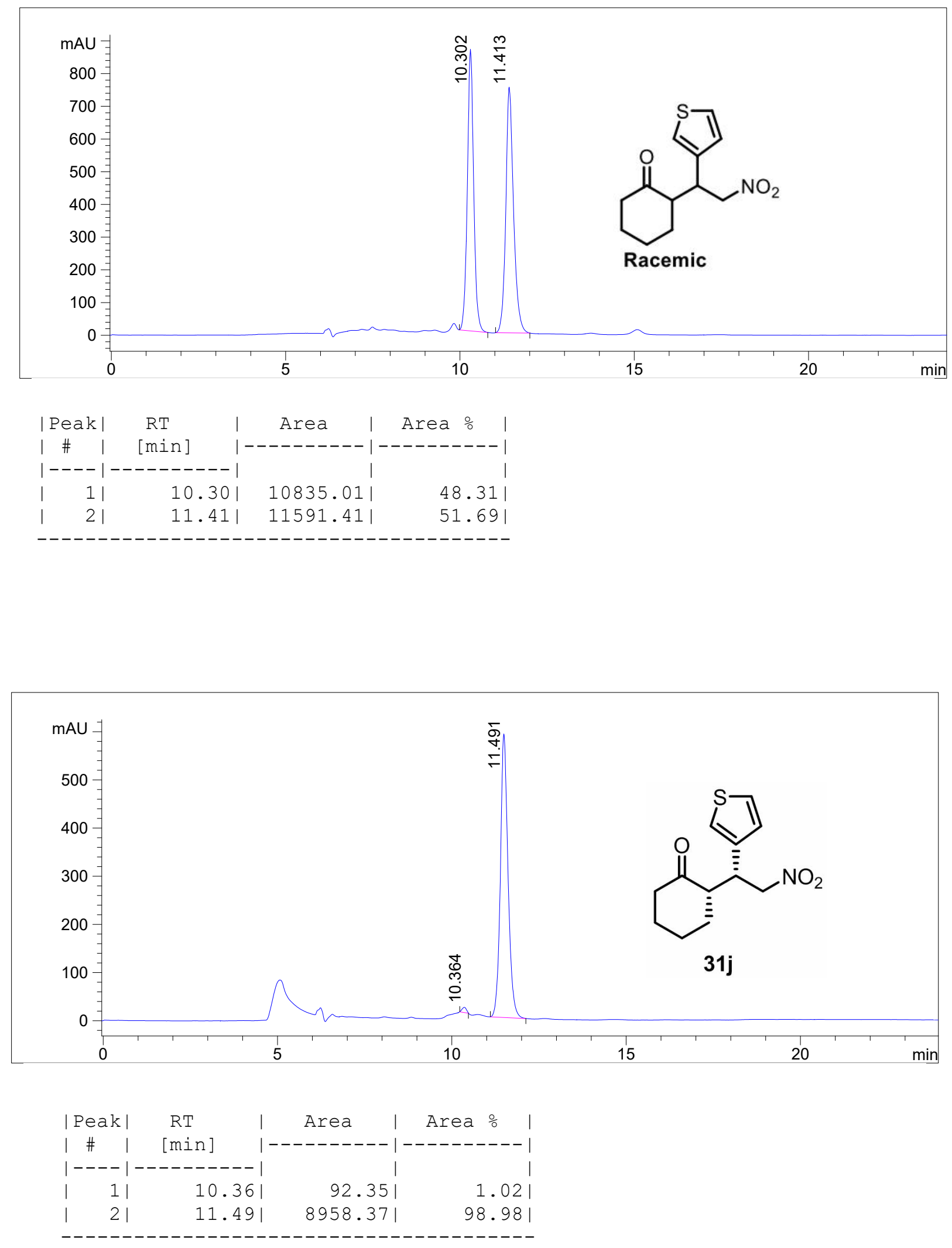
Column Name : Chiralpak IA (4.6 x $250 \mathrm{~mm}), 5 \mu$

Mobile phase : EtoH : 100

Flow Rate : $0.5 \mathrm{ml} / \mathrm{min}$

Solubility : $\mathrm{MeOH}$

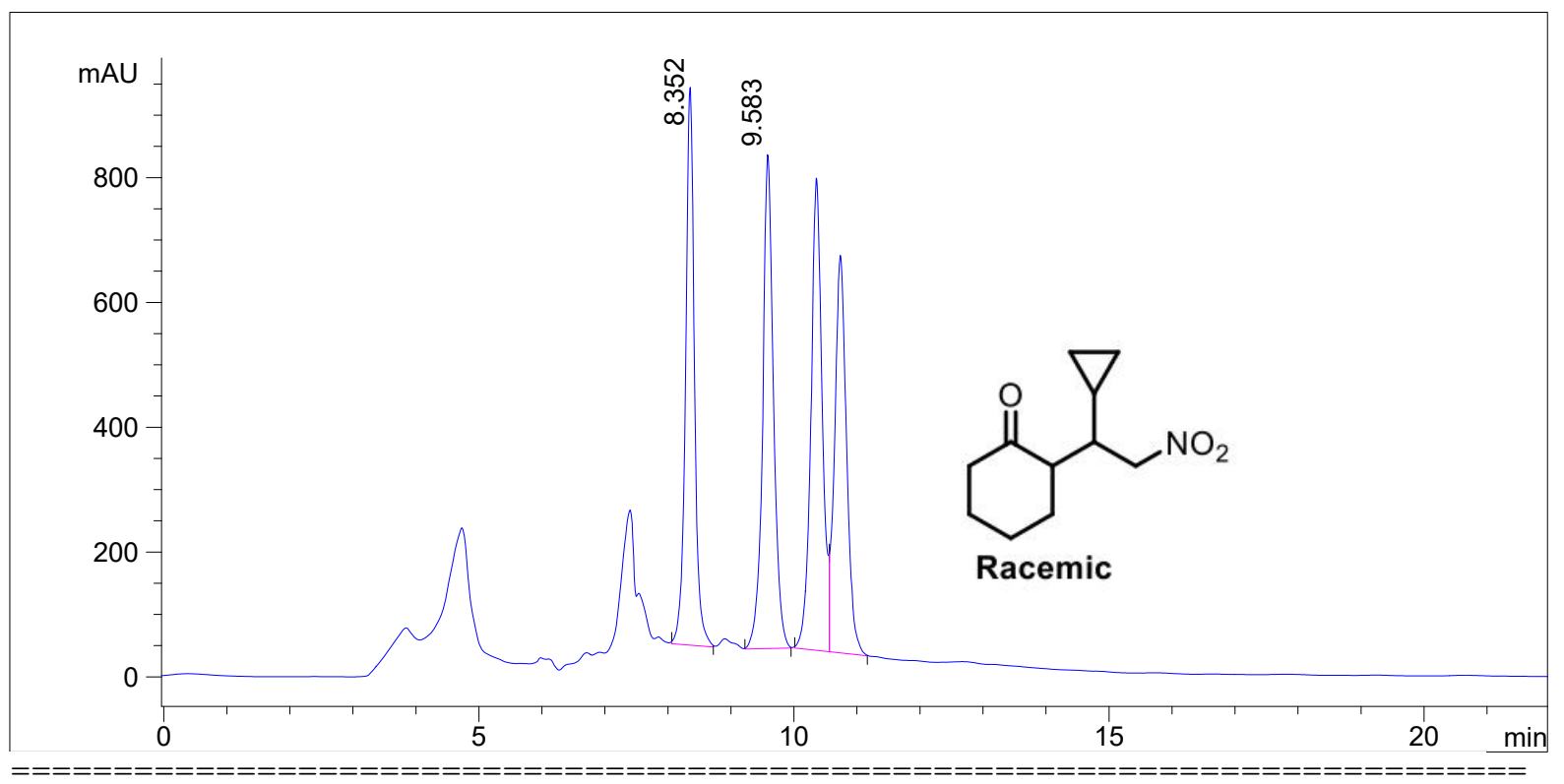

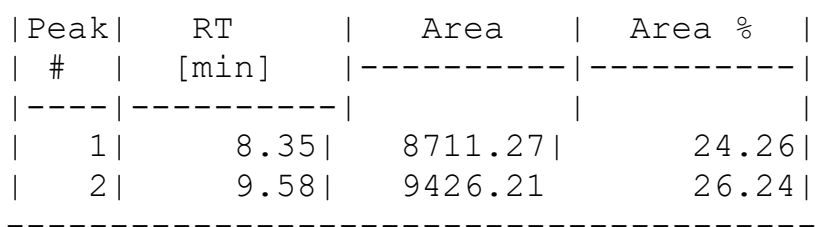
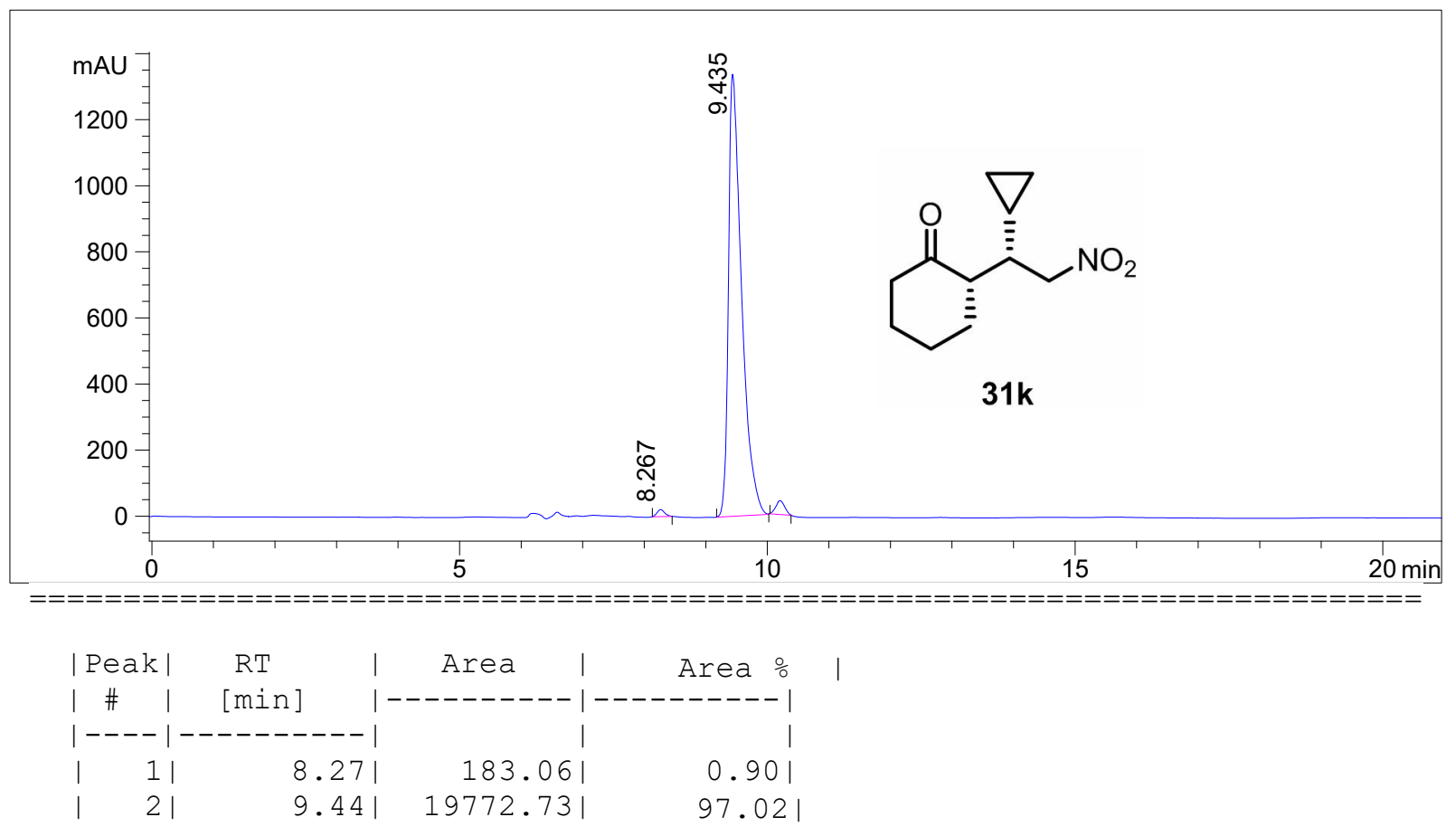
Column Name : Chiralpak IA (4.6 x $250 \mathrm{~mm}), 5 \mu$

Mobile Phase : EtOH : 100

Flow Rate : $0.5 \mathrm{ml} / \mathrm{min}$

Solubility : $\mathrm{MeOH}$
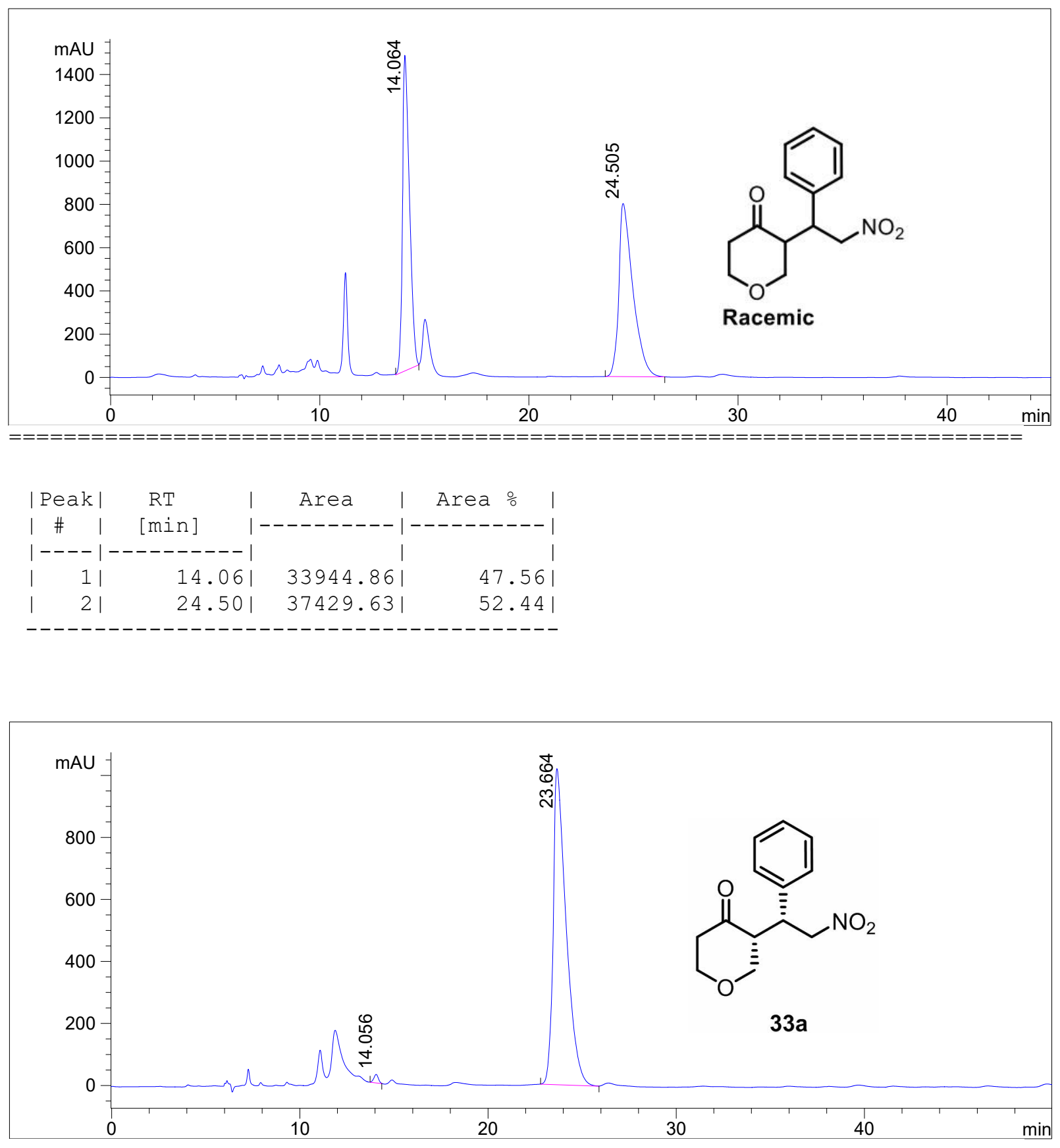

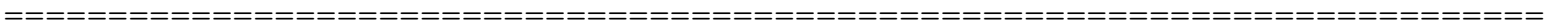

\begin{tabular}{|c|c|c|c|}
\hline | Peak | & RT & Area & Area \% \\
\hline | $\#$ | & [min $]$ & $---------\mid$ & $----------\mid$ \\
\hline$|----|$ & $--------\mid$ & | & \\
\hline | 1 | & 14.06 & $457.26 \mid$ & 0.931 \\
\hline 21 & 23.661 & 48726.741 & 99.071 \\
\hline
\end{tabular}


Column Name : Chiralpak IA (4.6 x $250 \mathrm{~mm}), 5 \mu$

Mobile Phase : EtoH : 100

Flow Rate : $0.5 \mathrm{ml} / \mathrm{min}$

Solubility : $\mathrm{MeOH}$

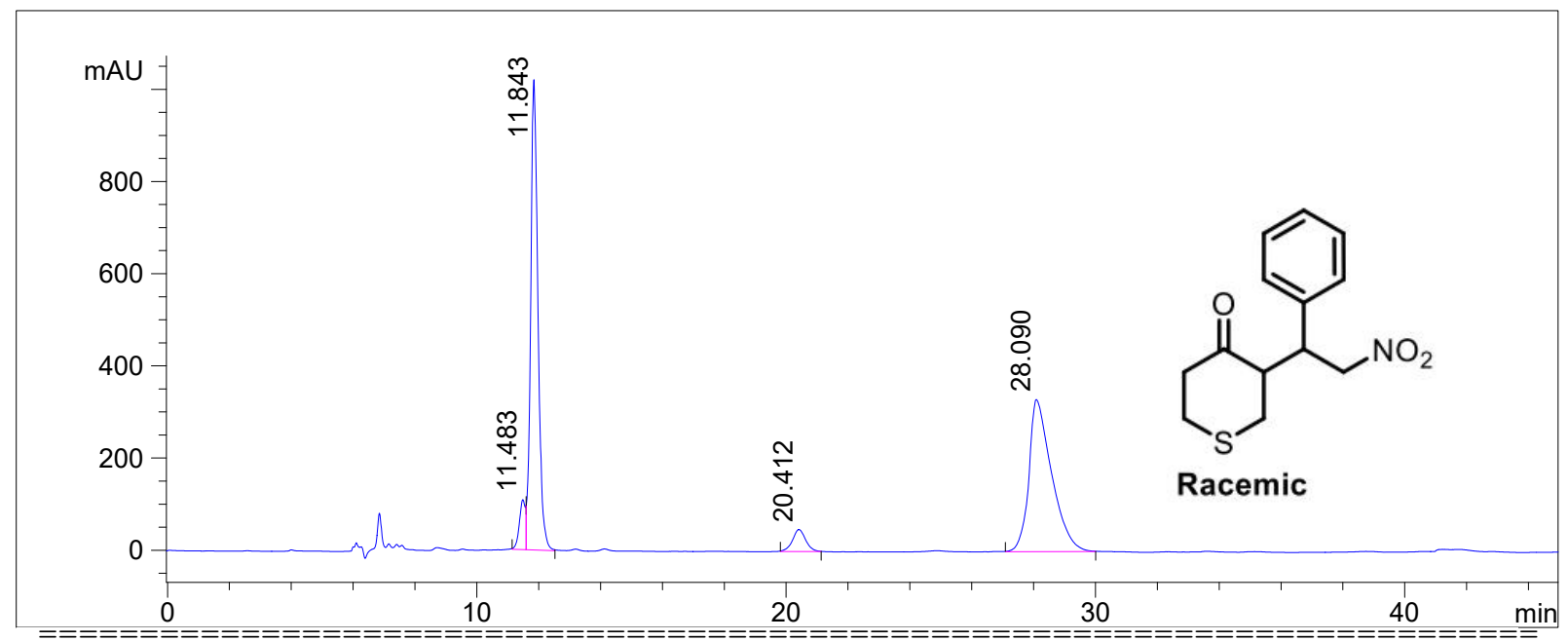

\begin{tabular}{|c|c|c|c|}
\hline | Peak | & $\mathrm{RT}$ & Area & ea $\frac{0}{\circ}$ \\
\hline | \# & [min] & $----\mid$ & ----1 \\
\hline$|----|$ & $---------\mid$ & | & \\
\hline | 1 | & 11.84 & 26589.241 & 45.641 \\
\hline 21 & 28.091 & 16848.431 & 46.351 \\
\hline
\end{tabular}

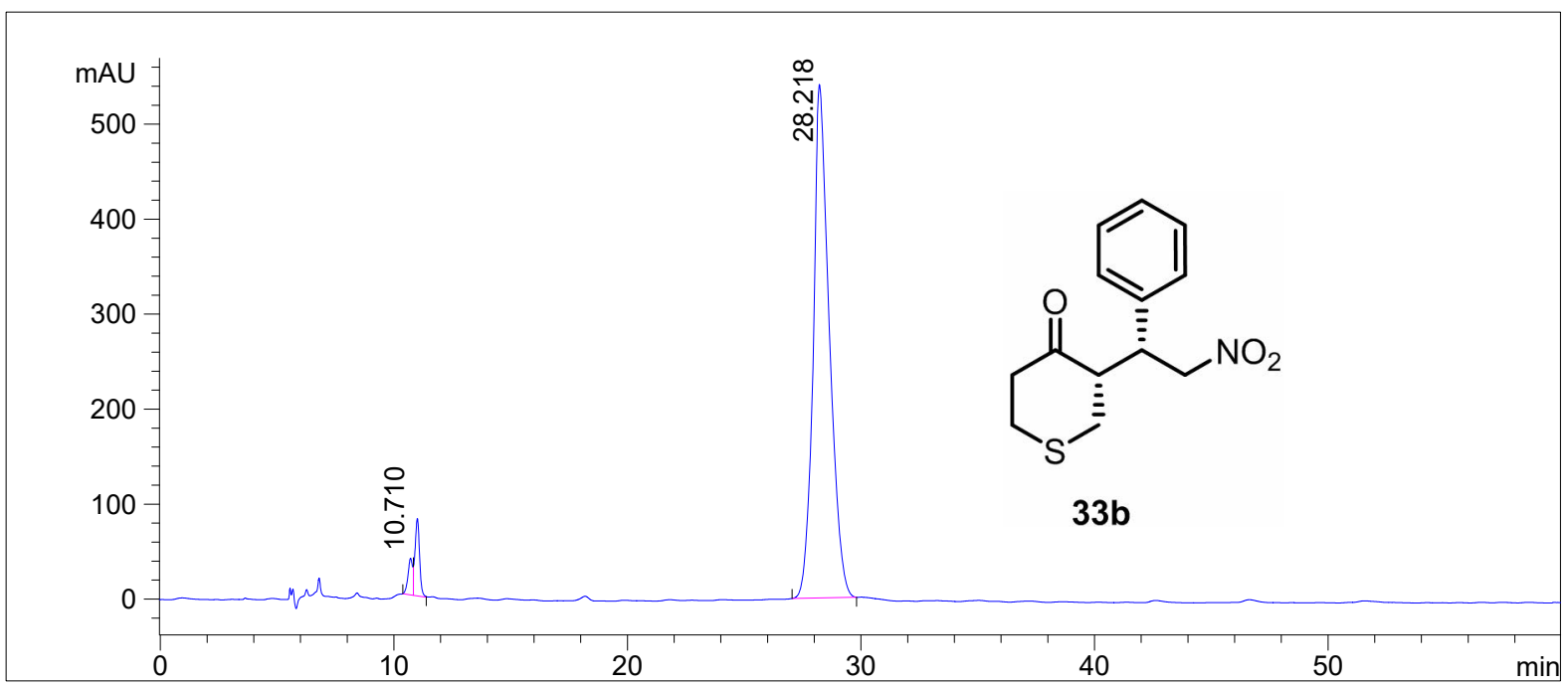

\begin{tabular}{|c|c|c|c|}
\hline | Peak | & $\mathrm{RT}$ & Area & $2 \div$ \\
\hline | \# $\quad$ | & [min] & $---------\mid$ & ----- \\
\hline$|----|$ & $---------\mid$ & & \\
\hline 11 & 10.71 & 540.001 & $1.97 \mid$ \\
\hline 21 & $11.00 \mid$ & $1112.76 \mid$ & 4.0 \\
\hline 31 & 28.221 & $25722.50 \mid$ & 93.96 \\
\hline
\end{tabular}


Column Name : Chiralpak IG (4.6 x $250 \mathrm{~mm}), 5 \mu$

Mobile phase : Hexane/DCM/EtOH : 80/10/10

Flow Rate : $1.0 \mathrm{ml} / \mathrm{min}$

Solubility : $\mathrm{MeOH}$

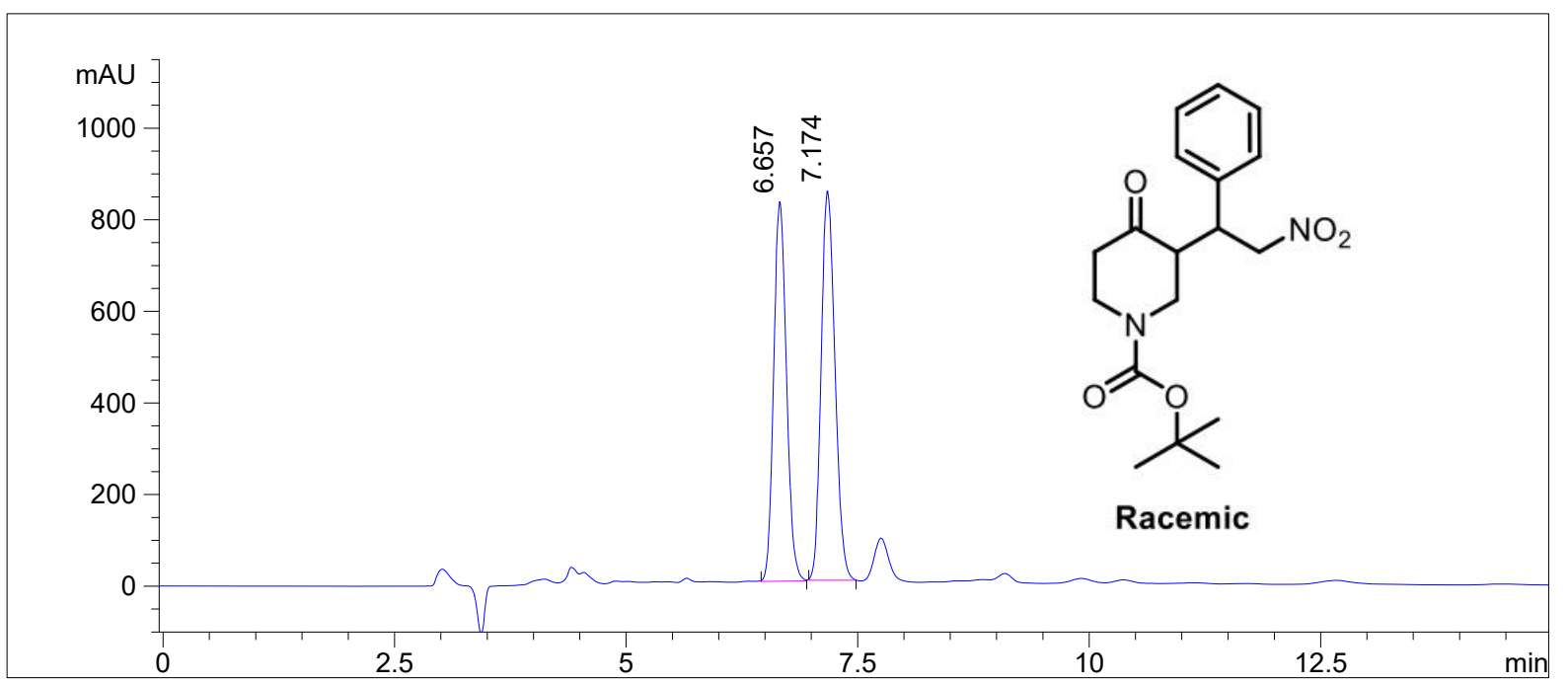

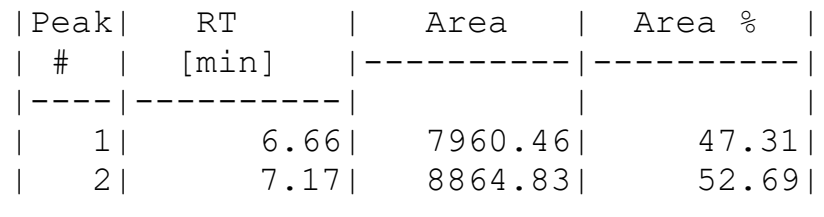

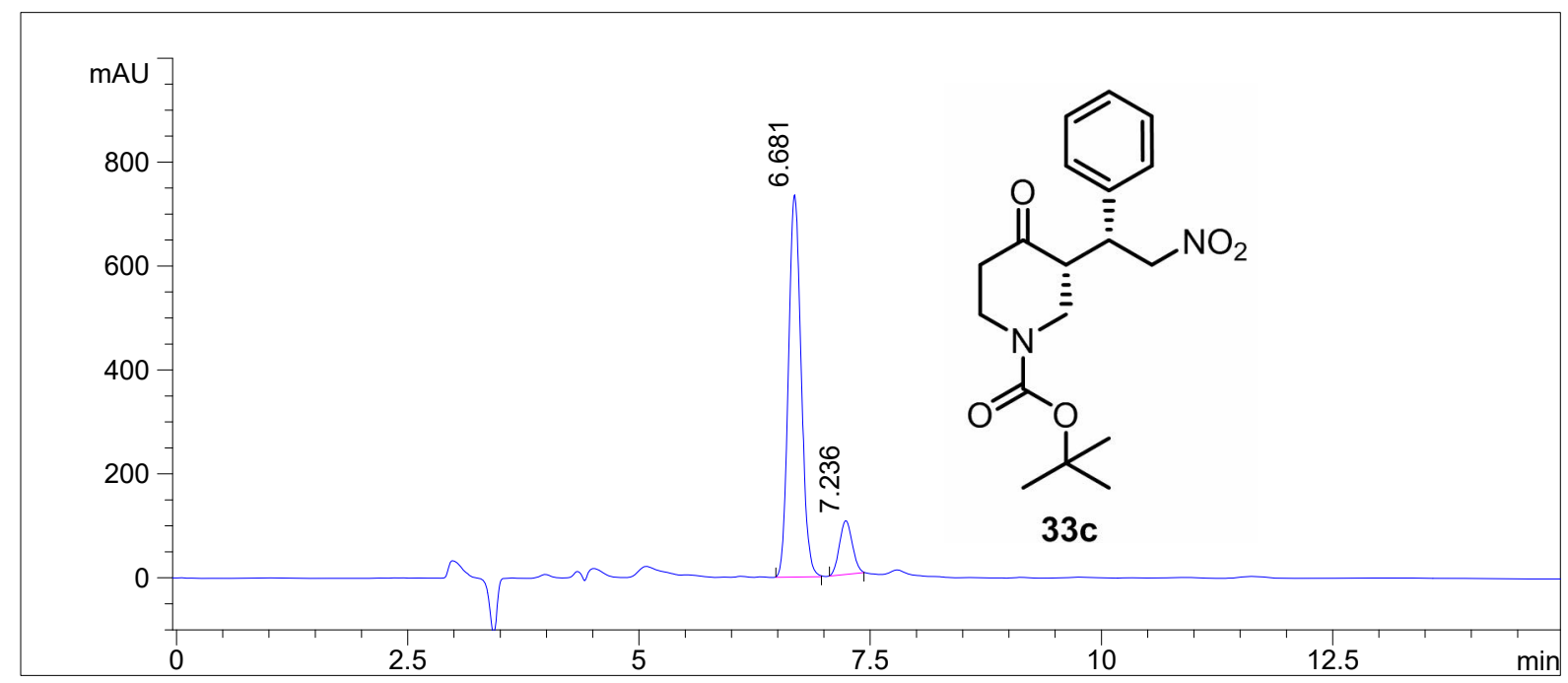

\begin{tabular}{|c|c|c|c|}
\hline | Peak | & $\mathrm{RT}$ & Area & $2 a$ \\
\hline | $\#$ | & [min] & $--------\mid$ & $------\mid$ \\
\hline$|----|$ & $---------\mid$ & | & \\
\hline | 1 | & 6.68 & 7043.311 & 87.591 \\
\hline 21 & 7.241 & $997.54 \mid$ & 12.41 \\
\hline
\end{tabular}




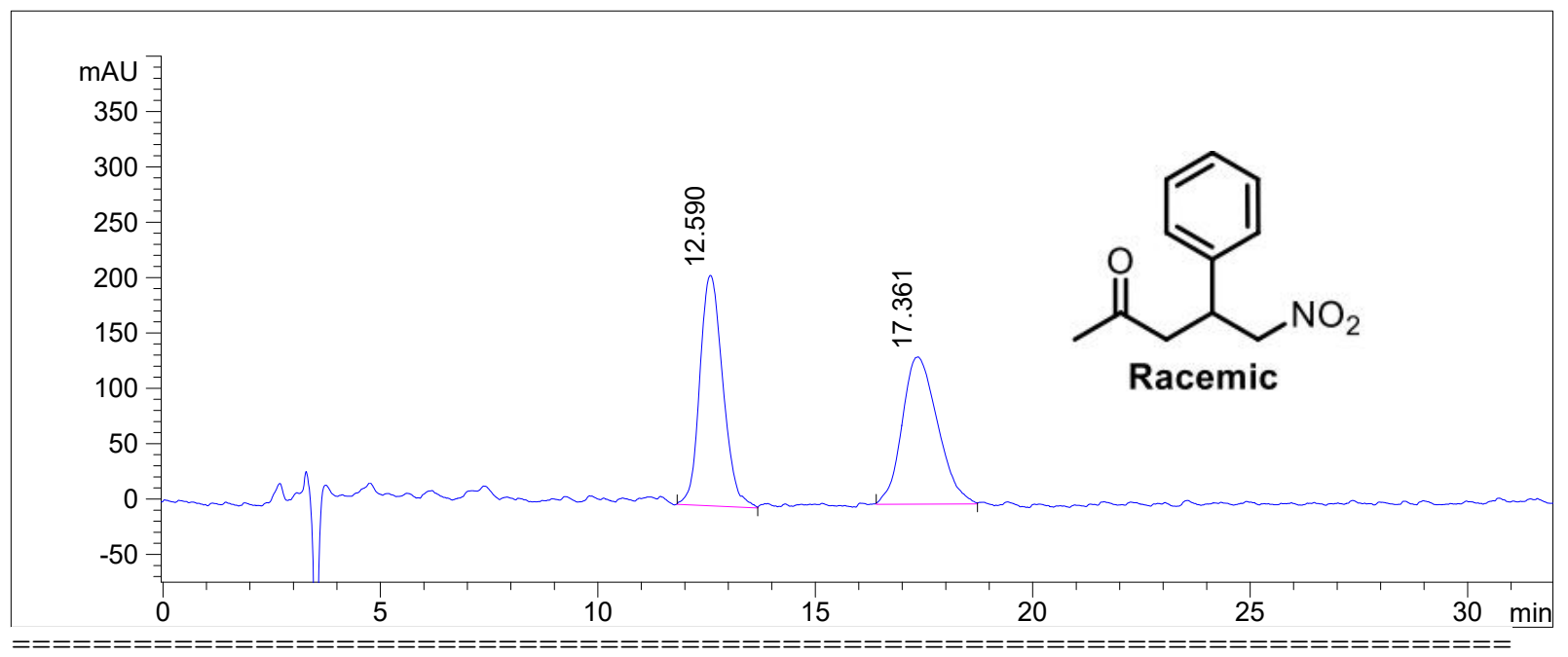

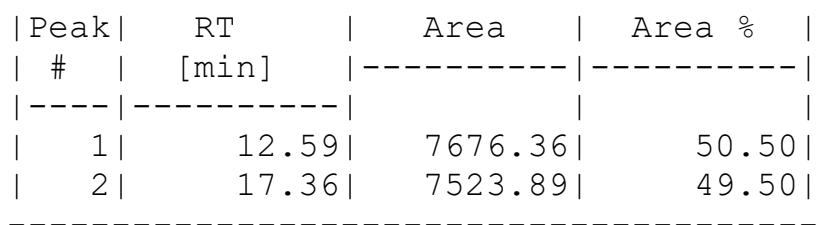

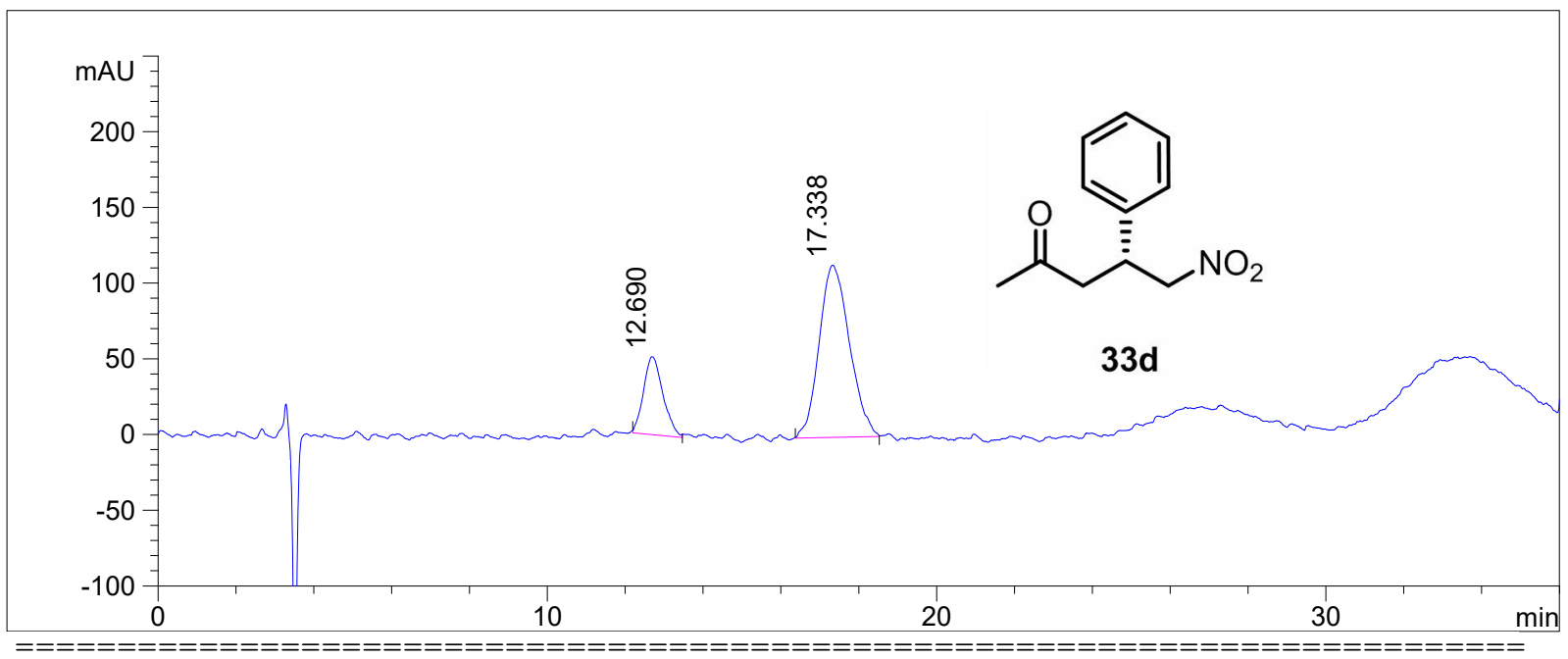

\begin{tabular}{|c|c|c|c|}
\hline | Peak | & $\mathrm{RT}$ & Area & ea $\frac{\circ}{0}$ \\
\hline | $\#$ \# 1 & [min] & -----1 & -----1 \\
\hline$|----|$ & $---------\mid$ & | & \\
\hline$|\quad 1|$ & 12.69 & $1778.49 \mid$ & 22.411 \\
\hline 21 & $17.34 \mid$ & 6156.001 & 77.591 \\
\hline
\end{tabular}


Column Name : Chiralcel OD-H (4.6 x $250 \mathrm{~mm}), 5 \mu$

Mobile phase : Hexane/IPA: $80 / 20$

Flow Rate : $1.0 \mathrm{ml} / \mathrm{min}$

Solubility : $\mathrm{MeOH}$

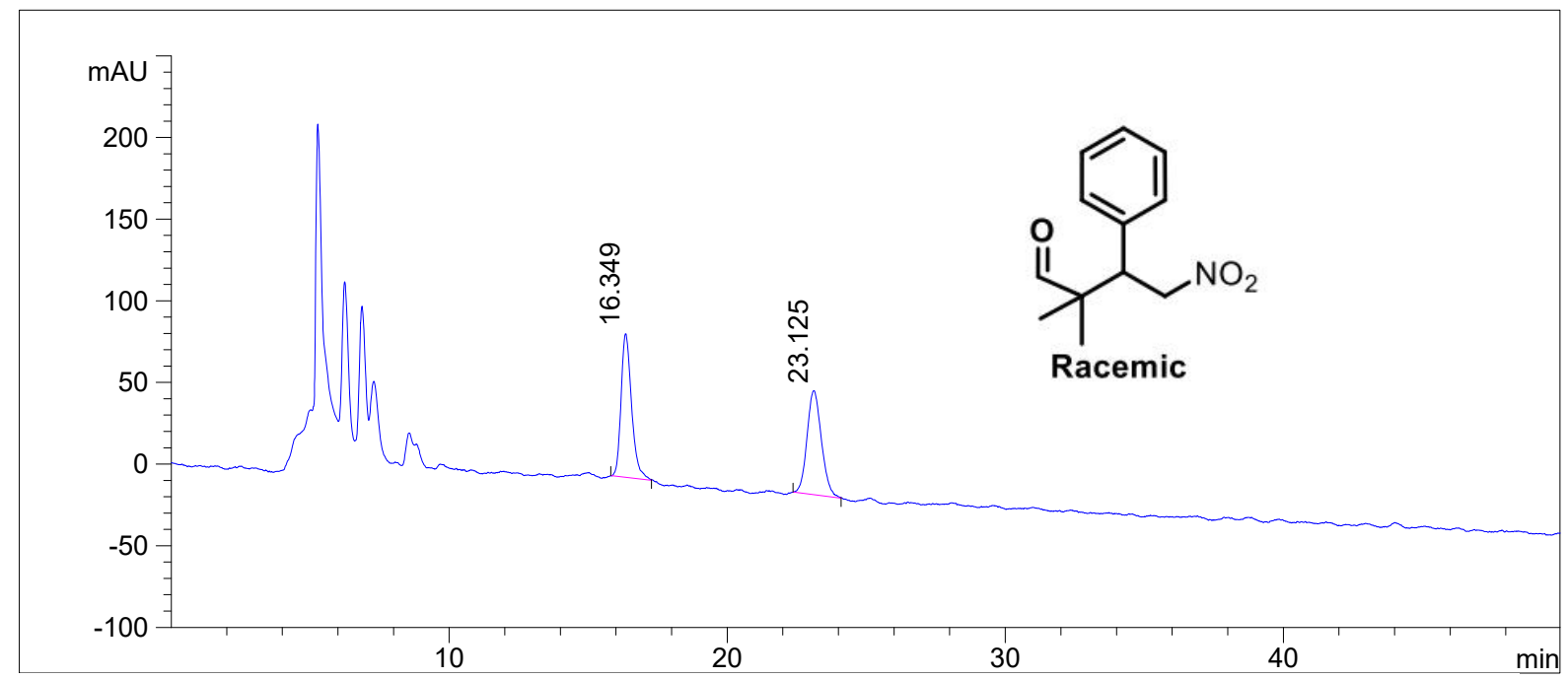

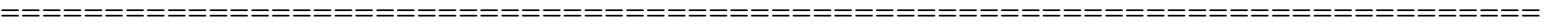
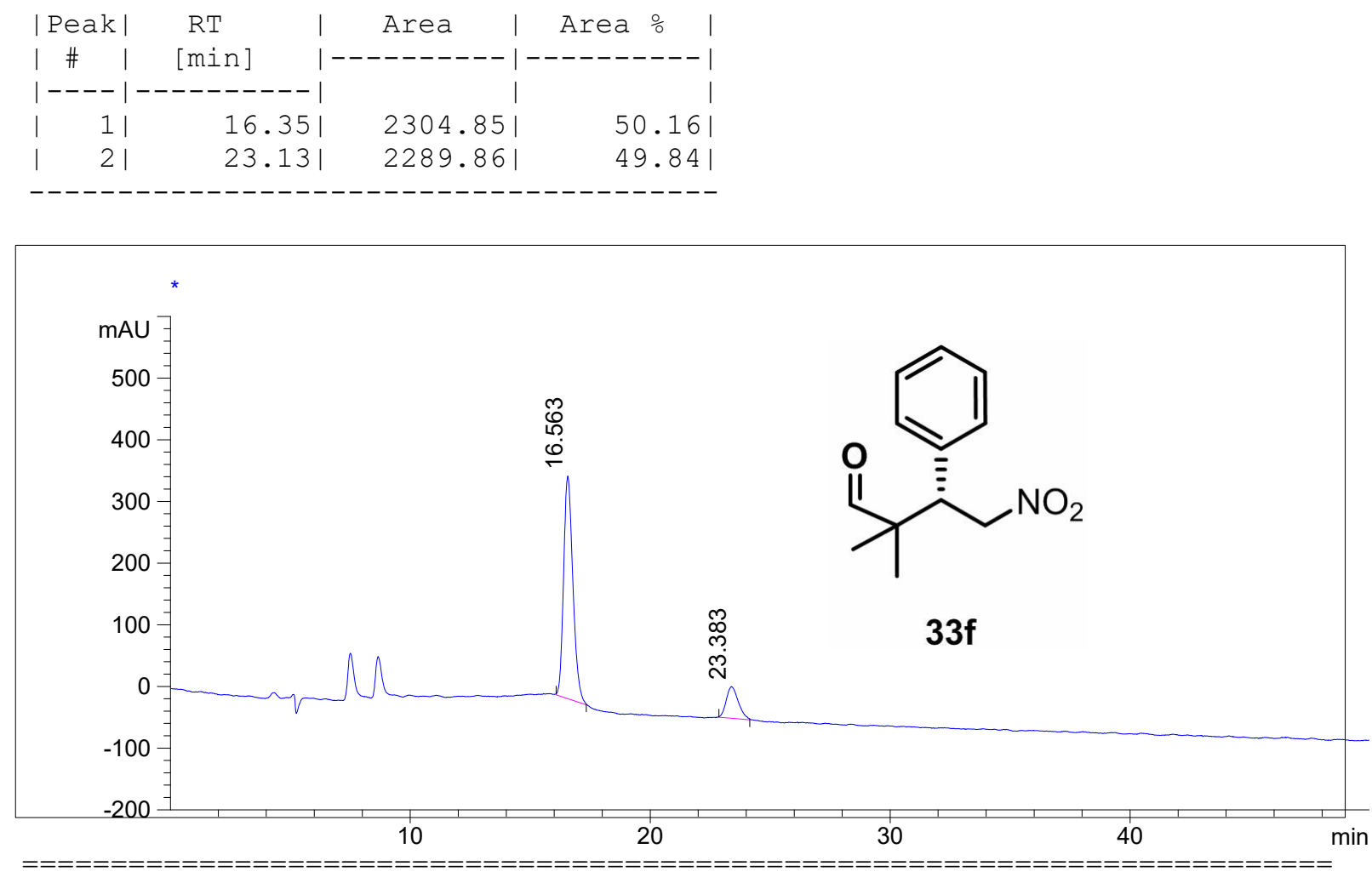

\begin{tabular}{|c|c|c|c|}
\hline $\mid$ Peak | & $\mathrm{RT}$ & Area & $2 \%$ \\
\hline | $\#$ I & [min $]$ & $------\mid$ & ---- \\
\hline$|----|$ & $--------\mid$ & | & \\
\hline | 1 | & 16.56 & 9667.831 & $84.37 \mid$ \\
\hline 21 & 23.381 & 1791.07 | & 15.63 \\
\hline
\end{tabular}




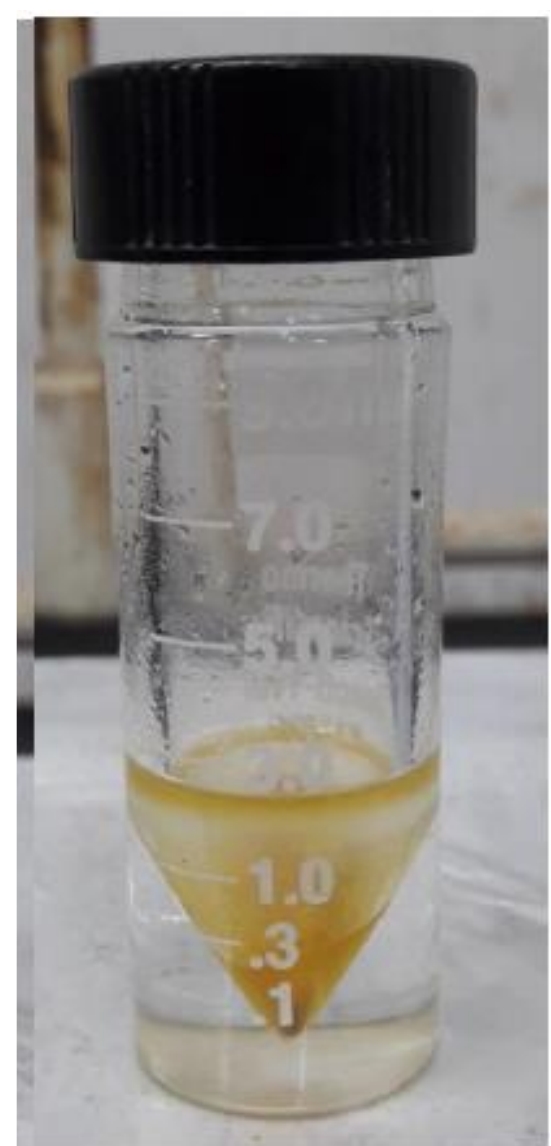

(a)

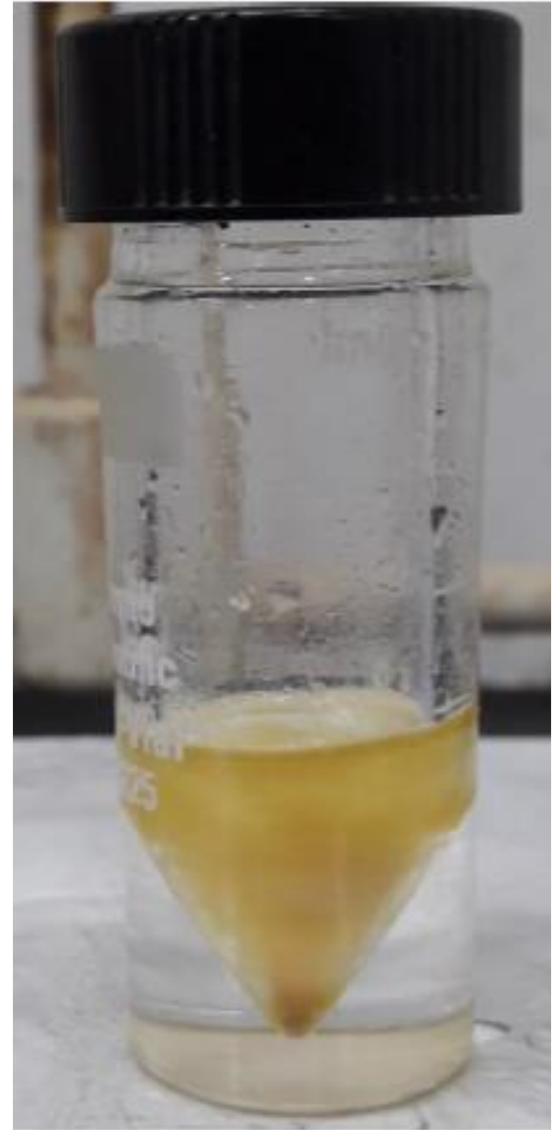

(b)

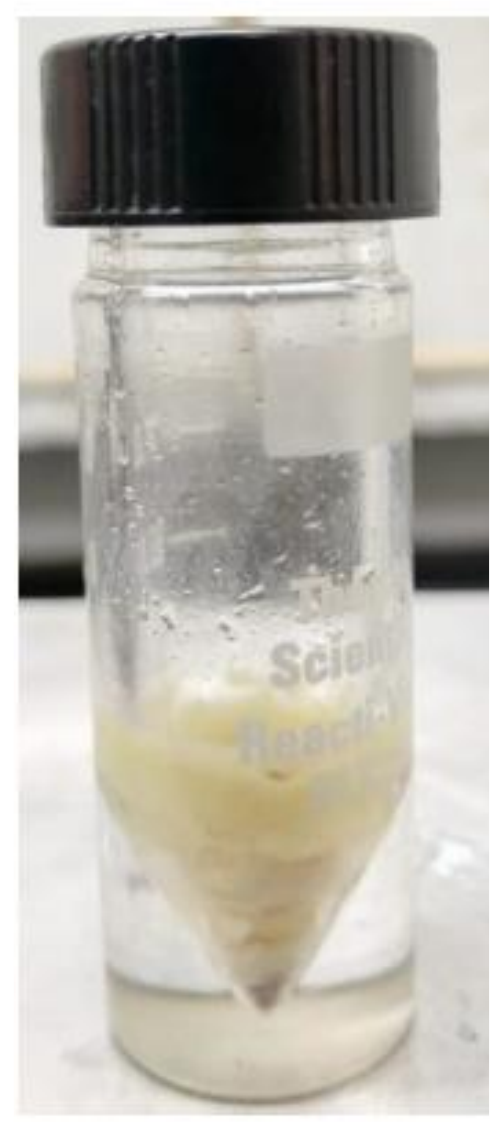

(c)

Figure S1. Images of various stages of the reaction involving the formation adduct 31a with cat. 1: (a) At the starting of the reaction, clearly showing two separate layers of water and organic part; (b) Formation of emulsion during the reaction under stirring; (c) After completion of the reaction, the insoluble product as precipitate in water. 